\title{
SELF-ASSEMBLED PEPTIDE NANOSTRUCTURES FOR ELECTRICAL, OPTICAL, AND MAGNETIC APPLICATIONS
}

A Dissertation
Presented to
the Faculty of the Graduate School
at the University of Missouri-Columbia
In Partial Fulfillment
of the Requirements for the Degree
Doctor of Philosophy
boma Khanra
Dr. Suchismita Guha, Dissertation Supervisor
May 2018


The undersigned, appointed by the dean of the Graduate School, have examined the dissertation entitled

SELF-ASSEMBLED PEPTIDE NANOSTRUCTURES FOR ELECTRICAL, OPTICAL, AND MAGNETIC APPLICATIONS

presented by Soma Khanra

A candidate for the degree of Doctor of Philosophy

and hereby certify that, in their opinion, it is worthy of acceptance.

Professor Suchismita Guha

Professor Ping Yu

Professor Gavin King

Professor Tommi White 
This work is dedicated to my parents who never ceased to believe in my abilities, my uncle whose constant care hedge me against all vicissitudes of my fortune, my husband whose ability to encourage me never waned despite his absence from this country, and my mentor; Dr. Suchismita Guha whose talent imbued with passion and love imbibed with dedication are etched in every page of this thesis. 


\section{ACKNOWLEDGEMENTS}

I am wholeheartedly grateful to my mentor Dr. Suchismita Guha for her sincere efforts in helping me during my Ph.D. I would not have accomplished my research work without her continual supervision and guidance. I am indebted to her for her support at each stage of my Ph.D. I would also like to thank Dr. Kartik Ghosh from Missouri State University for his insightful contribution towards my research work.

I would like to thank Dr. Ping Yu for his invaluable suggestions on my second harmonic generation project. I would specially like to thank Thiago Cipriano from Federal University of $\mathrm{ABC}$ (UFABC), SP, Brazil and Dhanushree Moghe who helped me in initiating my research work. Further, I acknowledge the support from our collaborator Drs. Alves, Ferreira from UFABC and Dr. Fileti from ICT-UNIFESP. I would also like to acknowledge Dr. Glaser from the Chemistry department for his invaluable suggestions to understand the chemistry of the molecular structures. I would like to thank my committee

members: Dr. Suchismita Guha, Dr. Ping Yu, Dr. Gavin King, and Dr. Tommi White for their suggestions and advice during my research. I can't forget to thank my friends and colleagues: Nag, Raghu, Madhu, Garima, Saru, Sanchita, Grant, Amrit, and Alec. It would not have been easy to stay here without their support.

I should not forget to acknowledge the contribution of my parents, uncles, sisters, and brothers who supported me all the way to here. I would have not been here without their sincere efforts. I would like to thank my aunt Keya Karmakar and cousin Krishan for helping me enjoy my graduate student life. Finally, I would love to express my gratitude to God for being with me. 
TABLE OF CONTENTS

ACKNOWLEDGEMENTS....................................................ii

LIST OF FIGURES...........................................................vii

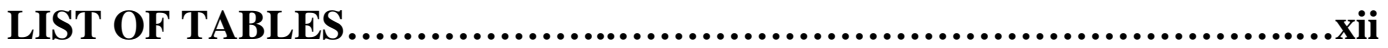

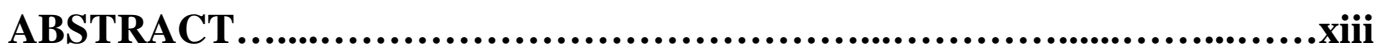

1 Motivation / Research Objectives/Introduction................................1

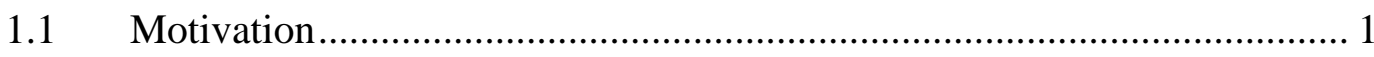

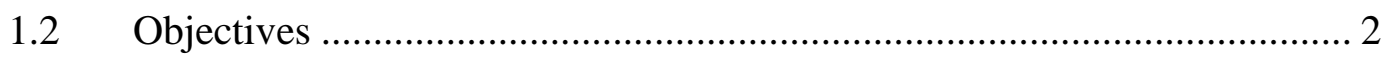

$1.3 \quad$ Introduction to Peptide Nanostructures (FF-MNS) ................................. 4

2 Experimental and Theoretical Methodologies.................................11

2.1 Structural Characterization Techniques .................................................... 11

2.1.1 X-Ray Diffraction (XRD) ....................................................... 11

2.1.2 X-Ray Photoelectron Spectroscopy (XPS) …………………….... 14

2.1.3 Scanning Electron Microscopy (SEM) ……………………….... 15

2.2 Optical Characterization Techniques...................................................... 17

2.2.1 Absorption Spectroscopy ……………………….................... 17

2.2.2 Photoluminescence Spectroscopy …………………………....... 18

2.2.3 Fourier Transform Infrared Spectroscopy (FTIR) ........................ 20

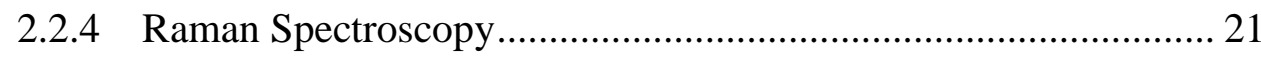

2.3 Second Harmonic Generation Experimental Setup ................................. 25

2.4 Magnetic Characterization Technique: SQUID Magnetometer ............... 27

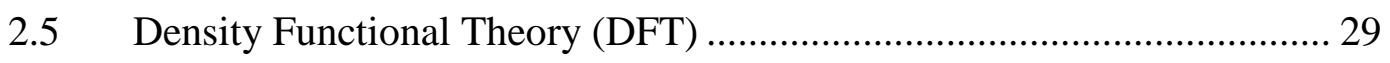




\section{Solvent Driven Structural Changes in Self-Assembled FF-MNS}

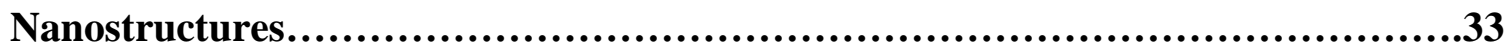

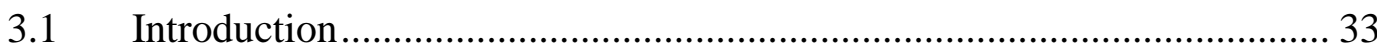

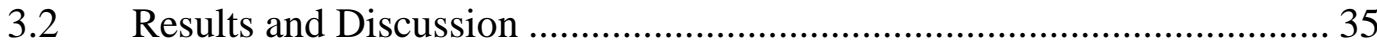

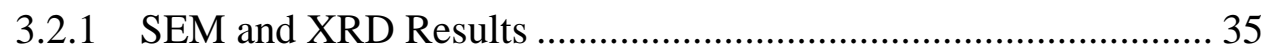

3.2.2 Raman Spectroscopy with Varying Solvents................................ 37

3.3 Theoretical Calculation ....................................................................... 40

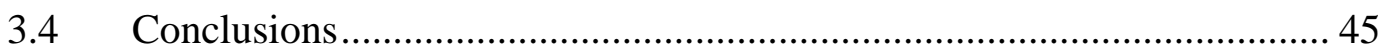

4 Probing Nonlinear Properties of FF-MNS Using SHG......................47

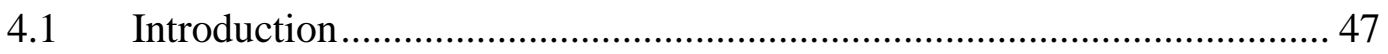

4.2 Symmetry and d Tensor in FF-MNS .................................................. 48

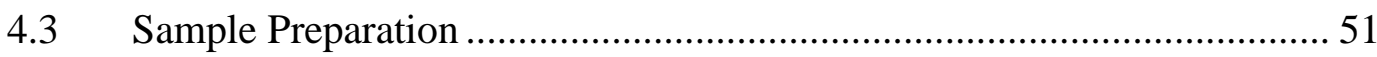

4.4 SHG Polarimetry as a Function of Tube Diameter ................................... 51

4.5 SHG Polarimetry as a Function of Thermal Annealing ..................... 56

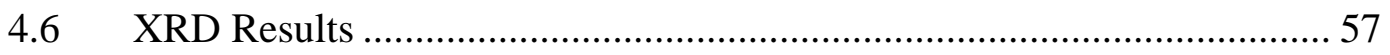

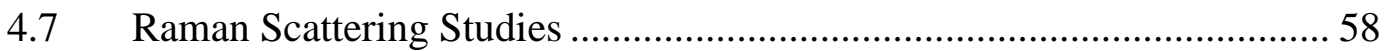

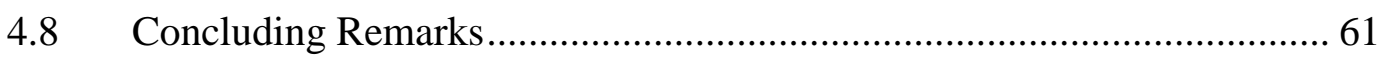

$5 \quad$ Functionalization of Peptide Nanostructures with Nanoparticles..............63

5.1 Functionalization of FF-MNS with Ag and Au Nanoparticles ................. 64

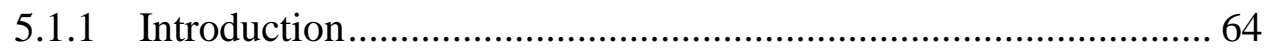

5.1.2 Synthesis of AgNps+FF-MNSs and AuNps+FF-MNSs ............... 65

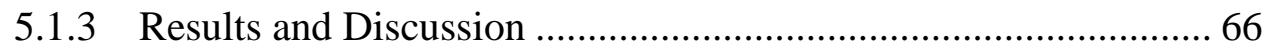

5.1.3.1 SEM and TEM Characterization ..................................... 66 
5.1.3.3 SERS from FF-MNS:AgNps Nanocomposites ............. 71

5.1.4 DFT Calculations ................................................................ 74

5.1.5 SERS from FF-MNS:AuNps .............................................. 78

5.1.6 Summarization of the SERS Results.................................... 81

5.2 Functionalization of FF-MNS with Cobalt Ferrite Nanoparticles ............ 82

5.2.1 Introduction.................................................................... 82

5.2.2 Synthesis of FF-MNS:CFO and FET Device Fabrication ........... 83

5.2.3 Magnetism in CFO ............................................................ 84

5.2.4 Results ............................................................................ 86

5.2.4.1 XRD and Structure of CFO ..................................... 86

5.2.4.2 TEM and SEM ................................................... 88

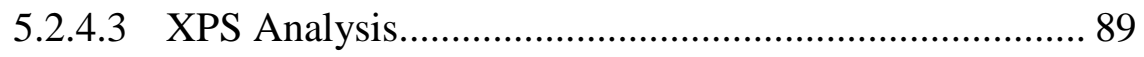

5.2.4.4 Raman Scattering and FTIR ................................... 95

5.2.4.5 Magnetization........................................................ 98

5.2.5 Discussion of Enhanced Magnetic Properties in FF-MNS:CFO 101

5.2.6 Application of FF-MNS:CFO in Organic Electronics .............. 105

5.2.7 Summarization of Functionalization of CFO with FF-MNS ..... 108

6 FF-MNS as Templates for Organic Light Emitting Diode (OLED)..........110

Introduction ......................................................................... 110

6.2 Synthesis of Peptide-polyfluorene Nanocomposite ............................ 113

6.3 Interaction Study of the Polymer with Peptide Nanostructure using SEM,

TEM, PL, Absorption Measurement and MD Simulation .............................. 114 
$6.4 \quad$ Composite Based OLED .............................................................. 119

6.5 Biodegradability Test................................................................. 121

6.6 Concluding Remarks.................................................................. 123

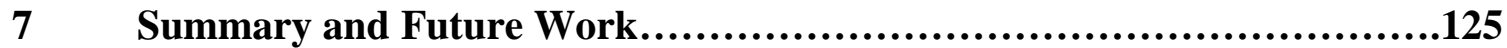

REFERENCES................................................................131

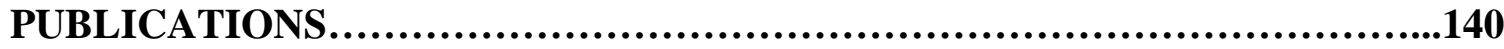

VITA 


\section{LIST OF FIGURES}

Figure 1.1. Application of peptide nanotubes. ${ }^{1}$.............................................................. 2

Figure 1.2. Formation of FF from amino acids through condensation reaction................. 5

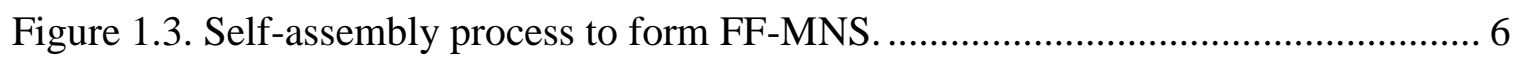

Figure 1.4. Nanostructures with various shape and size. $.^{3-4} \ldots \ldots \ldots \ldots \ldots \ldots \ldots \ldots \ldots \ldots \ldots \ldots \ldots \ldots \ldots \ldots . . .17$

Figure 1.5. Demonstrate different synthesis approach of peptide nanostructures. ${ }^{6}$........... 9

Figure 1.6. XRD data from FF-MNSs with Rietveld analysis in (a) hexagonal phase and (b) orthorhombic phase. Inserted images show the crystal structure packing obtained from XRD data. ${ }^{8}$ 10

Figure 2.1. XRD measurement system at Missouri State University................................ 14

Figure 2.2. Illustrating the working principle of the XPS.............................................. 15

Figure 2.3. Configuration of a typical SEM. .............................................................. 16

Figure 2.4. Electronic transitions in a molecule. .......................................................... 17

Figure 2.5. (a) A schematic diagram of energy levels in conjugated molecules and polymers. (b) Electronic ground and excited states in configuration space. ${ }^{14}$................ 19

Figure 2.6. Schematic diagram of the experimental set up for photoluminescence

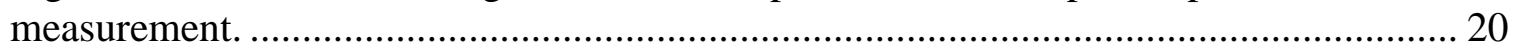

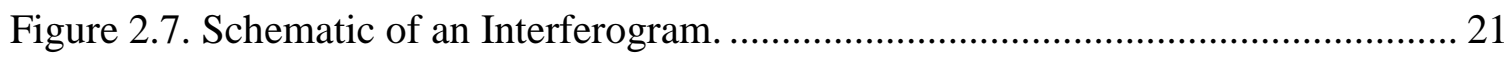

Figure 2.8. Energy level of a molecule showing different types of Raman signal.......... 22

Figure 2.9. (a) Symmetric stretching vibration, (b) Asymmetric stretching vibration, (c) Bending vibration, and (d) Bending vibration........................................................... 24

Figure 2.10. Schematic representation of Raman Microscope......................................... 25

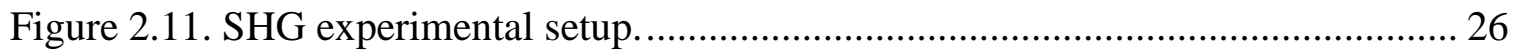

Figure 2.12. Schematic diagram of a SQUID magnetometer showing working principle of

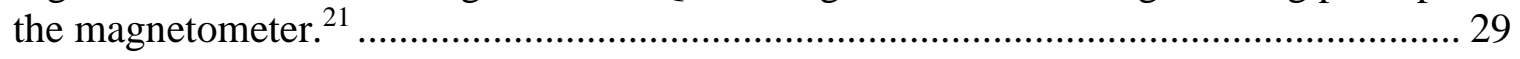

Figure 3.1. Molecular structure of (a) toluene. (b) water, (c) DMSO, and (d) PC. .......... 35

Figure 3.2. SEM images of FF-MNs synthesized using (a-b) toluene, (c-d) DI water, (e-f)

DMSO, and (g-h) PC. ………………………………....................................... 36 
Figure 3.3. XRD pattern of FF-MNS nanostructures synthesized using different solvents.

Figure 3.4. Raman spectra from FF-MNs nanostructures in (a) $65-400 \mathrm{~cm}^{-1}$, (b) $400-800$

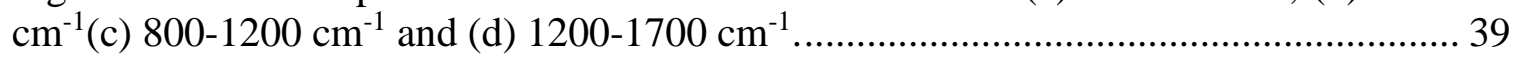

Figure 3.5. (a) Shows the arrangement of three basic unit of peptide nanostructure in the crystal of the peptide nanostructure Solvation model used for DFT calculation............. 41

Figure 3.6. . (a) and (b) Calculated Raman spectra. (c) Table reporting the bond lengths of FF molecule optimized in varying solvents...................................................... 45

Figure 4.1. Schematic of the lab frame and the sample frame...................................... 49

Figure 4.2. (a-e) SEM images of individual FF-MNTs showing the outside diameters. (f) SEM image of a FF-MNT with an overall length of $148 \mu \mathrm{m}$. (g) Experimental SHG polarimetric response from two FF-MNTs with different outer diameters for p-polarization. ( $\mathrm{h}-\mathrm{j}$ ) Experimental SHG response from three different outer tube diameters: $5.8 \mu \mathrm{m}, 8.5 \mu \mathrm{m}$, and 13.2 $\mu \mathrm{m}$. In each case the sample was oriented such that the laboratory frame and the sample's reference frame coincided. The black symbol and dotted line correspond to s-polarization and the red symbol and dotted line correspond to p-polarization. 53

Figure 4.3. (a) Modulus of $d_{31} / d_{33}$ as a function of the edge length of hexagonal FF-MNTs. The inset shows an SEM image of a cross-section of FF-MNT. (b) p-polarization experimental data (black Square) and a fit (red line) using eq ${ }^{\mathrm{n}}$ (4.12). (c) Total SHG intensity as a function of the incident power of the fundamental frequency. The bold line is a fit with square law dependence. (d) $d_{15} / d_{33}$ ratio as a function of the edge length of individual FF-MNTs. (e) Total SHG intensity as a function of the polarization angle of the incident light. Black squares are the experimental results and the red line is a fit to the overall intensity by combining equation (4.11) and (4.12). (f) SHG intensity (s-polarization) as a function of the sample orientation for FFMNTs with O.D. $8.5 \mu \mathrm{m}$ and $13.2 \mu \mathrm{m}$. The solid line is a fit to the square of equation (4.9). 54

Figure 4.4. (a) SHG response of a FF-MNT (with approximate O.D. of $4 \mu \mathrm{m}$ ) after annealing at temperatures of $100{ }^{\circ} \mathrm{C}$ (square), $125^{\circ} \mathrm{C}$ (circle), and $143{ }^{\circ} \mathrm{C}$ (triangle). (b) $d_{15} / d_{33}$ ratio of the FF-MNT obtained by fits to $\mathrm{eq}^{\mathrm{n}}$ (4.11) and (4.12) as a function of the annealing temperature. (c) $d_{31} / d_{33}$ ratio of the FF-MNT obtained by fits to $\mathrm{eq}^{\mathrm{n}}(4.12)$ as a function of the annealing temperature. 56

Figure 4.5. A schematic of the change in the domain and crystallite size as a function of the annealing temperature. 58

Figure 4.6. (a) Experimental and calculated Raman spectra from FF. The inset shows the structure of the FF molecule. (b) FF macrocycle with water molecules in the core. 59

Figure 4.7. (a) Raman spectra as a function of the tube diameter. (c) FF macrocycle with water molecules in the core. (b) Raman spectra of a FF-MNT (O.D. $=11 \mu \mathrm{m})$ as a function of annealing temperature. 
Figure 5.1. SEM images of the FF-MNS composites in the hexagonal $P 6_{1}$ structure with different amount of AgNps. a) Sample 0 without AgNps; b) Sample 3; c) and d) are from sample 5. e) and f) TEM images of Sample 5 shows the AgNps to aggregate on the hexagonal surface.

Figure 5.2. SEM images of the FF-MNS composites in the orthorhombic $P 22{ }_{1} 2_{1}$ structure with different amount of AgNps. a) Sample 0 without AgNps; b) Sample 3; c) Sample 5. d), e) and f) are TEM images of Sample 5, showing the AgNps dispersed inside the orthorhombic structure. 68

Figure 5.3. SEM images of the FF-MNS composites with AuNps (a) hex phase, and (b) orthorhombic phase.

Figure 5.4. UV-Vis of the solutions of AgNps and the FF-MNSs based composites, a) hexagonal FF-MNSs and b) orthorhombic FF-MNSs. FTIR spectra of the peptide assemblies with the c) hexagonal and d) orthorhombic crystal structure. There is no significant difference between the materials without and with AgNps. 71

Figure 5.5. a) SERS spectra of RhB from Sample 5 in the hexagonal and orthorhombic structure. The RhB concentration was $10^{-4}$ mol L ${ }^{-1}$. b) SERS spectra of the hexagonal samples with different amount of AgNps solutions (\%), from 0\% (sample 0) to $100 \%$ (sample 5). c) SERS spectra of sample 5 with different concentrations of $\mathrm{RhB}$, from $10^{-4}$ to $10^{-9} \mathrm{~mol} \mathrm{~L}^{-1}$. d) Calibration curve plot showing the exponential dependence of the concentration of $\mathrm{RhB}$ for the band at $1649 \mathrm{~cm}^{-1}$ 72

Figure 5.6. a) Hexagonal (hex) FF unit; b) Orthorhombic (ort) FF unit; c) R6G molecule; d) hex FF+Ag with R6G; e) ort FF+Ag with R6G. 75

Figure 5.7. Calculated Raman spectra of FF, FF+Ag, R6G and FF+Ag+R6G in the a) hexagonal and b) orthorhombic phase. The spectra are vertically scaled to depict the calculated Raman intensities. The color of the vertical axis corresponds to the color of the Raman spectra. All Raman cross sections are quoted in terms of $\mathrm{cm}^{2} / \mathrm{sr}\left(\times 10^{-31}\right) \ldots \ldots \ldots . . .77$

Figure 5.8. Raman spectra of pure R6G $10^{-4} \mathrm{~mol} \mathrm{~L}^{-1}$ (black) and R6G $10^{-7} \mathrm{~mol} \mathrm{~L}^{-1}$ attached to FF-MNS. a) hex FF-MNS+AuNps+R6G; b) ort FF-MNS+AuNps+R6G spectra...... 79

Figure 5.9. Experimental Raman spectra from FF-MNS, FF-MNS+AuNps, FFMNS+AuNps+R6G for the a) hexagonal and b) orthorhombic phase. The Raman spectrum of R6G+AuNps (without any FF-MNS) is shown for comparison in both panels. 81

Figure 5.10. The unit cell of $\mathrm{CFO}$ where $\mathrm{Fe}^{3+}$ atoms occupy both the octahedral $\left(\mathrm{O}_{\mathrm{h}}\right)$ and tetrahedral $\left(\mathrm{T}_{\mathrm{d}}\right)$ sites. The octahedral sites are also occupied by $\mathrm{Fe}^{2+}$, which are replaced by $\mathrm{Co}^{2+}$ in CFO. The black and the blue arrows point to the direction of the magnetic moment at the $\mathrm{T}_{\mathrm{d}}$ and $\mathrm{O}_{\mathrm{h}}$ sites, respectively. 86

Figure 5.11. (a) and (b) are the Rietveld plots of CFO and FF-MNS:CFO samples respectively. Open black circles represent the observed data; the red line indicates the 
calculated pattern, the blue line at the bottom displays the difference between observed and calculated data and the vertical magenta bars stand for the Bragg reflections.

Figure 5.12. (a) - (d) are the TEM images of CFO nanoparticles at various magnifications. (e) High resolution TEM image of FF-MNS:CFO. The yellow box shows the interface between CFO and FF-MNS:CFO, and its magnified image, with lattice fringes, is shown in (f). The inset is the Fourier transform of the image. (g) SEM image of a FF nanotube.

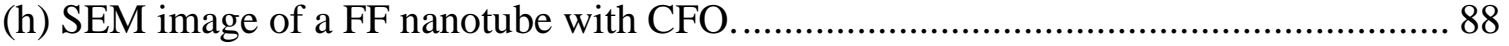

Figure 5.13. XPS spectra of FF-MNS, CFO, and FF-MNS: CFO ................................ 89

Figure 5.14. High-resolution X-ray photoelectron spectra of (a) C 1s, (b) N 1s, and (c) O 1s from FF-MNS:CFO ( left panel) and FF-MNS (right panel), and (d) Fe 2p in FFMNS:CFO (left panel) and CFO (right panel).................................................... 93

Figure 5.15. (a) and (b) Raman spectra of the predominant CFO vibrational modes in FFMNS:CFO and CFO, respectively. (c) and (d) the $1300 \mathrm{~cm}-1$ region in FF-MNS:CFO and $\mathrm{CFO}$, respectively. .96

Figure 5.16. FTIR spectra from FF-MNS and FF-MNS:CFO. The spectra in the range: 500 $\mathrm{cm}^{-1}$ to $4000 \mathrm{~cm}^{-1}$ are shown in (a) and (b). The high frequency region is shown in (b) . The region of the amide II and I bonds are shown in (c) and (d), respectively. 98

Figure 5.17. Magnetic hysteresis loops for CFO (black) and FF-MNS:CFO (blue) at room temperature. The inset shows the same data with the unit of magnetization in emu/g..... 99

Figure 5.18. Magnetization data for CFO and FF-MNS:CFO (high magnetic fields) at room temperature along with the fitted modified Langevin function as discussed below. The solid lines in (a) and (b) are the fits. 101

Figure 5.19. Schematic of the interaction model between CFO and the amine group in FFMNS. 105

Figure 5.20. Electrical characteristics of pentacene based MIS capacitors and FETs. FFMNS and FF-MNS:CFO served as the dielectric layer. (a) Schematic of MIS structures. (b) $\mathrm{C}-\mathrm{V}$ characteristics of MIS devices. The arrows denote the sweep directions. (c) Schematic of a bottom-gate, top-contact FET. (d) and (e) Output characteristics of pentacene FETs using FF-MNS and FF-MNS:CFO as the gate dielectric, respectively. The channel width to length ratio of the FETs was 10. 106

Figure 6.1. (a) Monomer unit of Polyfluorene. (b) Side chain of PF2/6. (c) Side chain of PF8. 112

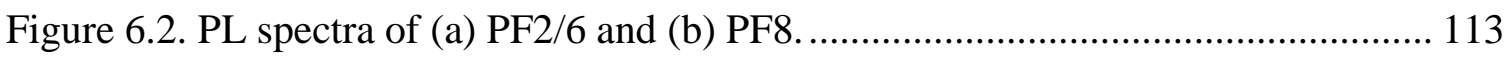

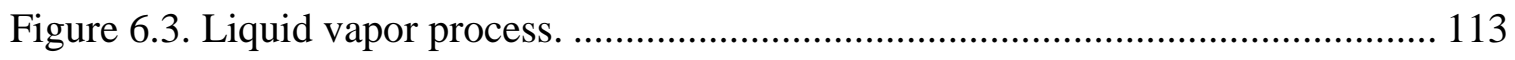


Figure 6.4. TEM image of (a) a FF nanotube and (b) FF:PF2/6. The dark regions in (b) show the aggregation of PF2/6.

Figure 6.5. (a) TEM image of FF:PF8 (as-is) nanocomposite. (b) A magnified image of a FF:PF8 (as-is) nanotube. (c) and (d) are representative TEM images of FF:PF8 (aged) nanotubes. In all images, the dark regions are the polymer and the lighter rod-like structure is the FF nanotube. 115

Figure 6.6. Absorption spectra of (a) PF2/6 and FF:PF2/6, (b) PF8 and FF:PF8 (as is), and (c) PF8 (aged) and FF: PF8(aged) (The peak at $435 \mathrm{~nm}$ depicted by the red arrow is a signature of the beta phase). PL spectra of (d) PF2/6 and FF:PF2/6, (e) PF8 and FF:PF8 (as is), and (f) PF8 (aged) and FF: PF8(aged). 116

Figure 6.7. (a) Panoramic view of the distribution of PF2/6 and PF8 monomers on nanotubes surface. 118

Figure 6.8. (a) shows the architecture of the OLED.(b) Image of a OLED device fabricated in our lab. 119

Figure 6.9. EL spectra of (a) PF2/6 and FF-MNS:PF2/6, and (b) PF8 and FF-MNS:PF8.

Figure 6.10. a) Percentage weight loss of FF-MNS, FF:PF8, and PF8 after incubation in buffer/enzyme system at $37{ }^{\circ} \mathrm{C}$ at different time intervals. b) Optical images of the three films before and after 2 and $6 \mathrm{~h}$ of incubation in buffer/enzyme solution. 122

Figure 7.1. Schematic presentation of the summary for this thesis. 125

Figure 7.2. Capacitance versus voltage from a pentacene MIS capacitor with no magnetic field (black square) and in the presence of $0.2 \mathrm{~T}$ magnetic field (red circles). Both $\mathrm{C}-\mathrm{V}$ curves were swept from positive to negative voltages. 129

Figure7.3. (a) Three FF units in FF-MNS crystal.(b) SEM image of Fluorinated FF nanostructures. 130 


\section{LIST OF TABLES}

Table 3.1. Dielectric constant and dipole moment of the used solvents........................... 35

Table 3.2. Lattice parameters from the nanostructures with varying solvents................. 37

Table 3.3. Raman peak position and identification of the vibration mode........................ 39

Table 3.4. Charge distribution of FF monomer unit in different solvents........................ 44

Table 3.5. List of minimum energy and entropy of FF monomer unit under varying

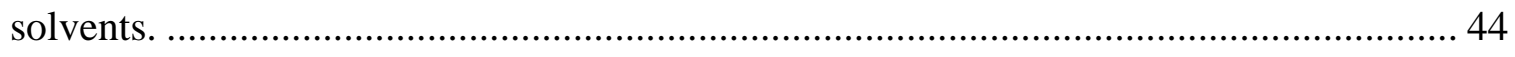

Table 4.1. Rietveld refinement parameters from FF-MNS............................................. 57

Table 5.1. Sample names and preparation for FF-MNS with AgNps............................. 65

Table 5.2. Experimental SERS peaks of RhB and assignment of the bands................... 73

Table 5.3. Dissociation energy of the Ag atom with respect to the FF unit.................... 75

Table 5.4. The peak positions and line widths of $\mathrm{C} 1 \mathrm{~s}, \mathrm{~N} 1 \mathrm{~s}, \mathrm{O} 1 \mathrm{~s}$, and $\mathrm{Fe} 2 \mathrm{p}$ by fitting the high resolution XPS data. The numbers in bold signify changes in the binding energy. . 94

Table 5.5. Fits from the modified Langevin-type function for CFO and FF-MNS:CFO. 100

Table 5.6. Magnetic moment of atoms for different configuration. ............................... 103

Table 5.7. Net magnetic moment for different configuration of $\mathrm{Fe}_{3} \mathrm{O}_{4}, \mathrm{CoFe}_{2} \mathrm{O} 4$, and FFMNS:CFO.

Table 6.1. Interaction energy of two nanocomposites. .............................................. 118 


\begin{abstract}
Bio-nanotechnology has become a widespread exciting field of research as the basic biological structure of bio-inspired materials and nanotechnology share the common length scale. Bio-nanotechnology, which is mainly based on bio-inspired nanostructured materials, has potential applications in nanomedicine, drug delivery, bio-sensors, and biodegradable electronic devices. The nanostructures obtained from biomolecules are attractive due to their biocompatibility for molecular recognition, ease of chemical modification, and the ability to scaffold other organic and inorganic materials. Peptide nanostructures formed through the self-assembly process of the basic building block of diphenylalanine show promising applications in biodegradable electronic devices, drug delivery, catalysis agent, waveguide, and frequency converter.

This research focusses on the self-assembly process in a dipeptide, $L, L$ diphenylalanine (FF) and exploring its electronic, optical, and magnetic properties. The role of solvents in the self-assembly process of FF is explored by combining density functional theory (DFT) along with experimental characterization techniques such as electron microscopy, Raman scattering, and x-ray diffraction (XRD).

One of the objectives of this work was to explore the nonlinear optical (NLO) properties of FF nanostructures via second harmonic generation (SHG). The ratio of the nonlinear optical coefficients was obtained from individual FF nanotubes as a function of the tube diameter and thermal annealing conditions. The ratio of the shear to the longitudinal component $\left(d_{15} / d_{33}\right)$ of the NLO coefficient increases with the diameter of the tubes. One of the transverse components, $d_{31}$, of the NLO coefficient is found to be negative, and its magnitude with respect to the longitudinal component $\left(d_{33}\right)$ increases with the tube diameter. Thermal
\end{abstract}


treatment of individual FF tubes has a similar effect as increasing the diameter of the tubes in SHG polarimetry.

The functionalization of FF micro-nanostructures (FF-MNS) with nanomaterials was studied. FF-MNS with Ag or Au nanoparticles were explored in surface-enhanced Raman scattering (SERS). Such self-assembled nanostructures provide a natural template for tethering Au and Ag nanoparticles (Nps) due to its fractal surface. The FF-MNS undergo an irreversible phase transition from hexagonal packing (hex) to an orthorhombic (ort) structure at $\sim 150{ }^{\circ} \mathrm{C}$. The metal Nps form chains on hex FF-MNS as inferred from transmission electron microscopy images and a uniform non-aggregated distribution in the ort phase. The SERS spectra obtained from R6G bound to FF-MNSs with AuNps show a higher enhancement for the ort phase compared with the hex phase. The experimental results agree well with our calculated Raman spectra of model systems using DFT. Our results indicate that FF-MNS both in the hex and ort phase can be used as substrates for SERS analysis with different metal Nps, opening up a novel class of optically active biobased substrates.

The use of magnetic nanoparticles with biomolecules offers a versatile path for tuning the functionality of the composite material for several applications. The functionalization of FF-MNS with cobalt ferrite (CFO) magnetic nanoparticles was achieved. The interaction between CFO nanoparticles and FF-MNS was investigated by optical spectroscopy, x-ray photoelectron spectroscopy (XPS), and magnetization measurements. The changes in the XPS data from pristine FF-MNS and CFO:FF-MNS are indicative of a charge transfer process from CFO to FFMNS, changing the electronic states of the $\mathrm{Fe}^{2+}$ and $\mathrm{Co}^{2+}$ ions. A comparison of the 
magnetic characterization from CFO nanoparticles and CFO:FF-MNS shows a higher saturation magnetization from the nanocomposite sample, which is attributed to a change in the cationic distribution in CFO upon binding with the peptide.

We were further successful in demonstrating the application of FF-MNS as a biodegradable active layer in an organic light emitting diode (OLED). FF-MNS were functionalized with two blue-emitting conducting polymers: di-octyl-substituted polyfluorene (PF8) and ethyl-hexyl polyfluorene (PF2/6), and used as an active layer in an OLED architecture. A combination of molecular dynamics and experimental characterization techniques reveals a stronger binding mechanism for PF8 compared to PF2/6 with FF-MNS. Biodegradability tests from FF-MNS:PF8 nanocomposite films show more than $80 \%$ weight loss in $2 \mathrm{~h}$ by enzymatic action compared to PF8 pristine films, which do not degrade. Self-assembled FF-MNS with organic semiconductors open up a new generation of biocompatible and biodegradable materials in organic electronics. 


\section{Motivation / Research Objectives/Introduction}

\subsection{Motivation}

Self-assembly is a spontaneous process of organization of molecular units into ordered nanostructures through intramolecular/intermolecular interactions such as non-covalent, van der Waals, electrostatic, hydrogen bonding, and stacking interactions. The formation of well-ordered nanostructures through self-assembly processes of diverse organic materials is the basic building block of modern nanotechnology. Nature can build the most remarkable structures ranging from the tiniest proteins to much more complex structures through self-assembly process. Self-assembly facilitates the achievement of various biological functions and can also be part of a pathogenic process. Examples of this dichotomy are the folding of polypeptide chains into useful functional proteins and the formation of amyloid fibrils that are associated with a number of diseases such as diabetes and Alzheimer's.

There are 20 naturally occurring amino acids, which form the basis of proteins. Peptides, which are biologically occurring short chains of amino acids, have versatile applications in materials science and technology.. L,L-diphenylalanine (FF), a short

dipeptide composed of two aromatic phenylalanine units, forms nanostructures through self-assembly process. FF-MNS) are attractive due to their unique physical and chemical properties. These include piezoelectricity, thermal and chemical stability, and relatively simple functionalization method. Figure 1.1 shows some of the applications of peptide nanostructures in electronics and other areas. In our work, we specifically target on electronic, photonic, and magnetic applications of FF nanostructures. 

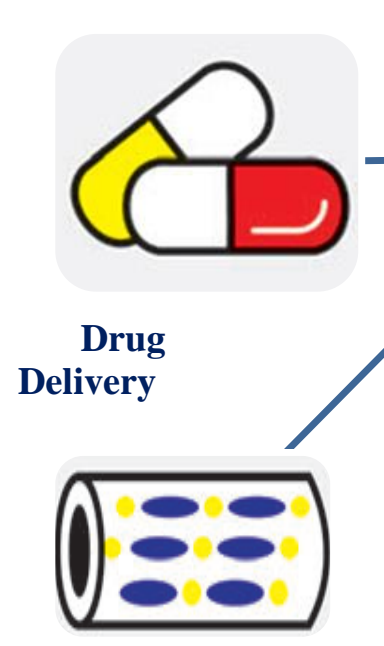

Scaffol

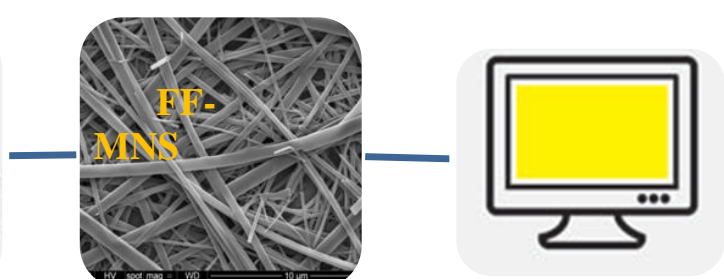

Displays and Light Emitter

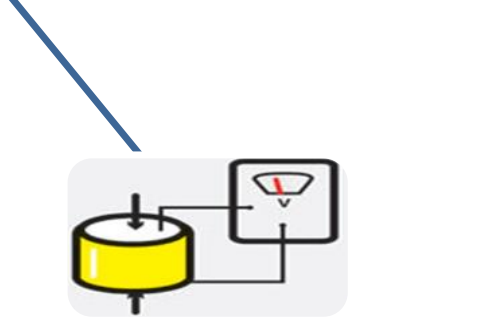

Piezoelectric

Ultra-Sensitive

Figure 1.1. Application of peptide nanotubes. ${ }^{1}$

\subsection{Objectives}

In this work, we focus on:

(1) The effect of the solvation on nanostrutures formation of FF using experimantal techniques and density functional based theortical calculation.

(2) Diameter dependent and temperature dependent nonlinear properties of FF .

(3) Functionalization of FF with nanoparticles such as $\mathrm{Ag}, \mathrm{Au}$, and cobalt ferrite (CFO) for electronic and magnetic applications.

(4) Utilize peptide nanostructures as a scaffold for polyfluorene based organic light emitting materials.

Here is an outline of how the thesis is structured.

Chapter 2 provides a detailed description of the experimental techniques used throughout my research work. It consists of the structural, optical, and magnetization 
characterization techniques used. At the end of this chapter, we describe the details of the DFT calculation.

Solvent driven self-assembly process of FF has been investigated through theoretical and experimental techniques in Chapter 3. This chapter starts with the optimized structural calculation of the FF monomer unit, which is the basic building block of peptide nanostructure, using DFT method. These calculations were conducted in solvents of varying dipole moments: DI water, toluene, propylene carbonate (PC) and dimethyl sulfoxide (DMSO) to investigate the structural changes of the FF monomer unit. Next, structural studies such as Scanning Electron Microscopy (SEM), Raman scattering, and XRD were conducted to understand the solvent driven self-assembly process in FF.

Exploitation of the structure dependent nonlinear optical (NLO) properties have been demonstrated in Chapter 4. We begin with the theoretical methodology for obtaining the nonlinear coefficients in second harmonic generation (SHG). We discuss the experimental data from diameter dependent and temperature dependent SHG measurements to determine the nonlinear coefficient. The Raman scattering, and temperature dependent XRD data have been analyzed to explain the change in the NLO properties with diameter and temperature.

In Chapter 5, we discuss the interaction of FF-MNS with nanoparticles. This chapter has two sections. In the first section, the interaction of FF-MNS with $\mathrm{Ag}$ and $\mathrm{Au}$ nanoparticles have been explored theoretically and experimentally for SERS application. In this section, the first part explains the synthesis of the Ag nanoparticles and FF-MNS. This is followed by characterization techniques including SEM, TEM, UV-Vis and FTIR to demonstrate the binding of the nanoparticles to the FF. The SERS effect was then 
demonstrated from the nanocomposite of $\mathrm{Ag}$ and dipeptide. Our DFT calculations shed light on the interaction mechanism and SERS effect. This section is followed by the SERS effect from Au nanoparticles with FF.

In the second section, the functionalization of FF-MNS with CFO magnetic nanoparticles was achieved. The interaction between CFO nanoparticles and FFMNS was then investigated by optical spectroscopy, x-ray photoelectron spectroscopy (XPS), and magnetization measurements. This was followed by using FF-MNS:CFO conjugate in field effect transistor (FET) for organic electronic applications.

In Chapter 6, the potential use of FF-MNS as scaffold for organic light emitting materials has been investigated. FF-MNS were functionalized with two blue-emitting conducting polymers: di-octyl-substituted polyfluorene (PF8) and ethyl-hexyl polyfluorene (PF2/6). This was followed by a combination of molecular dynamics and experimental characterization techniques including TEM, absorption spectroscopy, photoluminescence, and MD simulation to study interaction of FF-MNS with the polymers. OLED device fabrication using FF-MNS and polymer conjugated material was then accomplished. At the end of this chapter, we discuss the biodegradability of the FF-MNS and polymer conjugate.

Chapter 7 is a summary of the aforementioned chapters, and future perspectives of self-assembling peptides for multiferroic and optical materials.

\subsection{Introduction to Peptide Nanostructures (FF-MNS)}

The basic building block for FF-MNS is FF. Dipeptide is formed when two amino acids are bonded by a peptide bond through condensation process. In this process, two 
amino acids approach each other, with the acid moiety of one in proximity to the amino moiety of the other. One amino acid molecule loses $\mathrm{OH}^{-}$from its carboxyl group $(\mathrm{COOH})$ and the other loses $\mathrm{H}^{+}$from its amino group $\left(\mathrm{NH}_{2}\right)$. In this reaction, a water molecule and FF are produced. The reaction is represented in Figure 1.2.

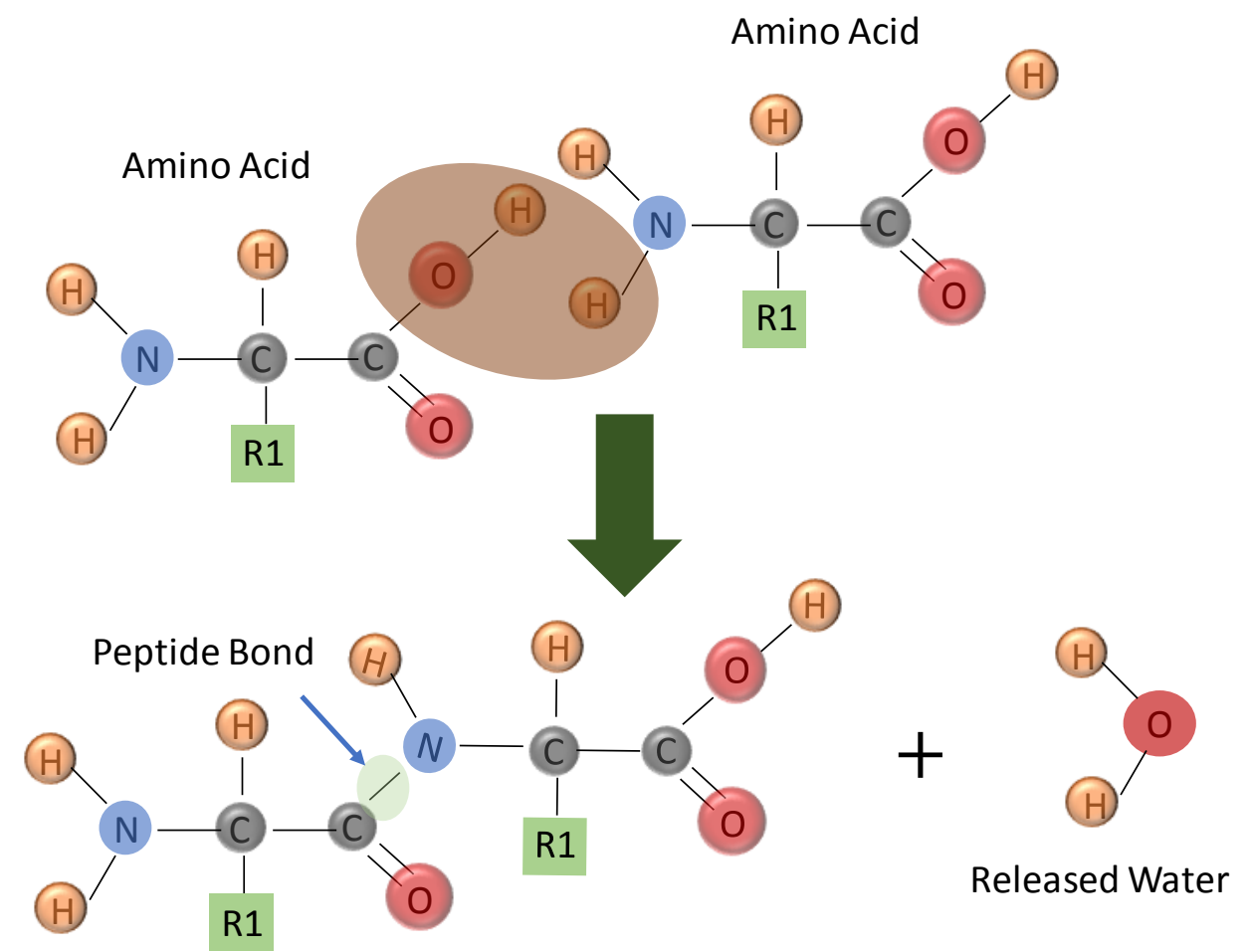

Figure 1.2. Formation of FF from amino acids through condensation reaction.

The ordering in FF-MNS begins at the molecular level with the association of different monomers (dipeptide), as illustrated in Figure. 1.3. Six FF monomers self-associate into 
macrocycle structures where amino and carboxyl groups constitute the inner core of the cycle.

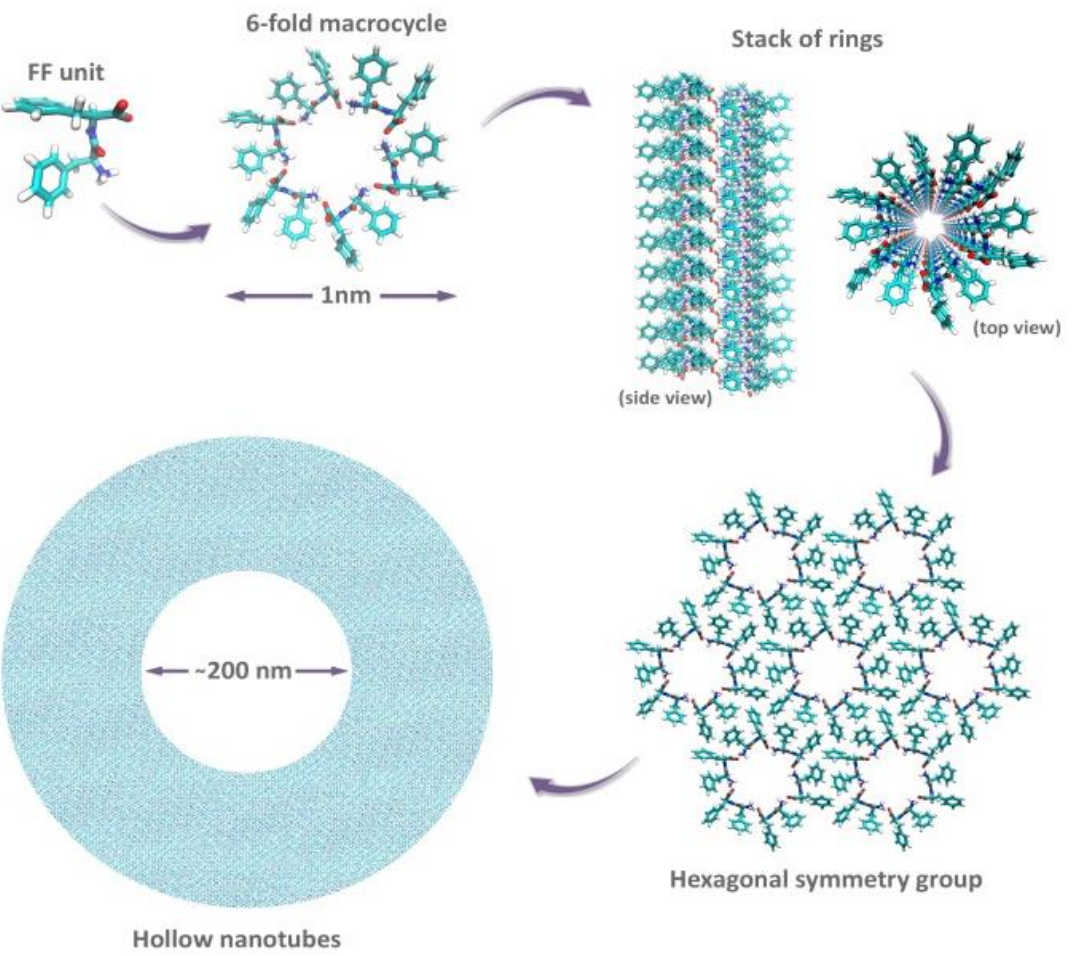

Figure 1.3. Self-assembly process to form FF-MNS.

The macro cycles stack into a columnar phase forming narrow tubes with diameter $\sim 1$ nm. These channels self-associate to make a hexagonal packing pattern and give rise to pleated sheets. ${ }^{2}$ The formation of nanotubes occurs by the closure of the sheets along the axes of the channels, leading to tubular arrays at the nanoscale. The self-assembly can achieve higher scales through successive associations where nanotubes form fibrillar bundles at micrometer lengths.

Self-assembling phenylalanine-based peptides can adopt diverse nanostructures such as nanotubes, nanorods, and nanospheres, shown in Figure 1.4, making them ideal as building blocks for many applications shown in Figure 1.1. 


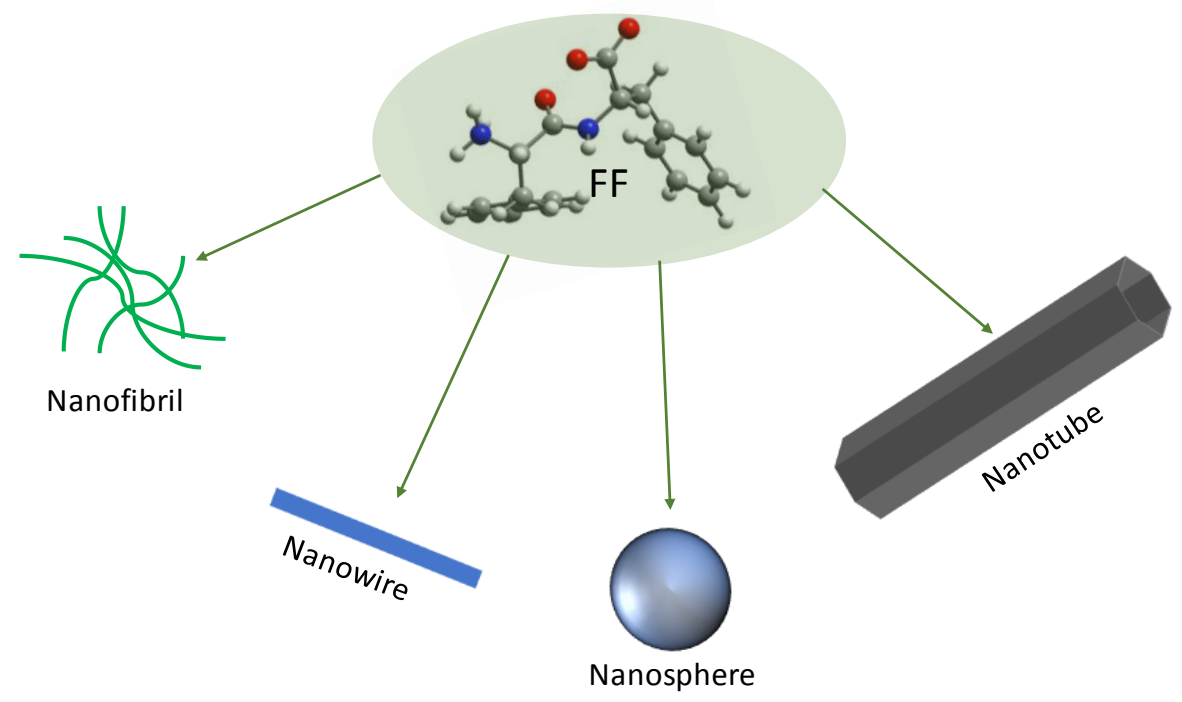

Figure 1.4. Nanostructures with various shape and size. ${ }^{3-4}$

Synthesis Process of FF-MNS Nanostructures

A schematic of various synthesis processes for FF-MNS is shown in Figure 1.5. ${ }^{5,6}$

\section{Solution Processed Peptide Nanostructures}

(a) Liquid Vapor: This is the simplest and most widely used approach to synthesize peptide nanostructures. In this method, FF is dissolved in HFIP and the stock solution is then diluted with water or other polar solvents to form nanostructures. In this work, $10 \mathrm{mg}$ of FF was dissolved in $100 \mu \mathrm{L}$ of HFIP solution (100 $\left.\mathrm{mg} \mathrm{mL}^{-1}\right)$ and then this solution was diluted by $450 \mu \mathrm{L}$ of DI water. This diluted mixture was immediately ultra-sonicated for about a minute at $10 \mathrm{kHz}$ frequency andkept in a refrigerator for about $8 \mathrm{~h}$ to complete the self-assembly process. The mixture was then centrifuged to separate the solid and liquid phase. The liquid phase was removed by a pipette from the separated solid-liquid mixture, and the solid phase was kept in an oven at $75^{\circ} \mathrm{C}$ until dry. 
(b) Solid Vapor: In this method, stock solution of FF monomer is prepared in HFIP and then spin coated on different substrate like aluminum, glass, or mica. Next, this spin-coated film is kept in isopropanol or alanine vapor at $80^{\circ} \mathrm{C}$ in a closed environment for 12 hours. This approach is the best approach for device application of peptide nanostructures. ${ }^{7}$

\section{i. Vapor Deposition Technique}

In this process, FF monomer powder is thermally evaporated. The FF powder is heated at $220^{\circ} \mathrm{C}$ and the substrate temperature is kept at $80^{\circ} \mathrm{C}$ in a vacuum environment with a chamber pressure of $1 \times 10^{-5}$ mbar. It forms vertically aligned nanostructures. This approach allows the length and density of the nanotubes to be fine-tuned by carefully controlling the supply of the building blocks from the gas phase. ${ }^{5}$ This technique can be used to develop high-surface-area electrodes for energy storage applications, highly hydrophobic self-cleaning surfaces and microfluidic chips.

\section{ii. Electrospinning of Peptide Nanostructures}

In this method, high voltage difference is applied between the needle from the reservoir containing the stock solution and the substrate. This high voltage forms a jet of solution in which the peptide monomers are present. Upon the evaporation of the HFP, peptide nanofibers are formed. The diameter of these fibers is more consistent compared to other processes. In addition, the diameters of the nanotube can be controlled to some extent by controlling the applied voltage and the concentration of the stock solution. This process requires a subtle balance between solution, solution concentration, viscosity, surface 
tension, applied voltage, and flowrate. Being difficult to control the parameters for proper injection of the solution, this approach has limitation.

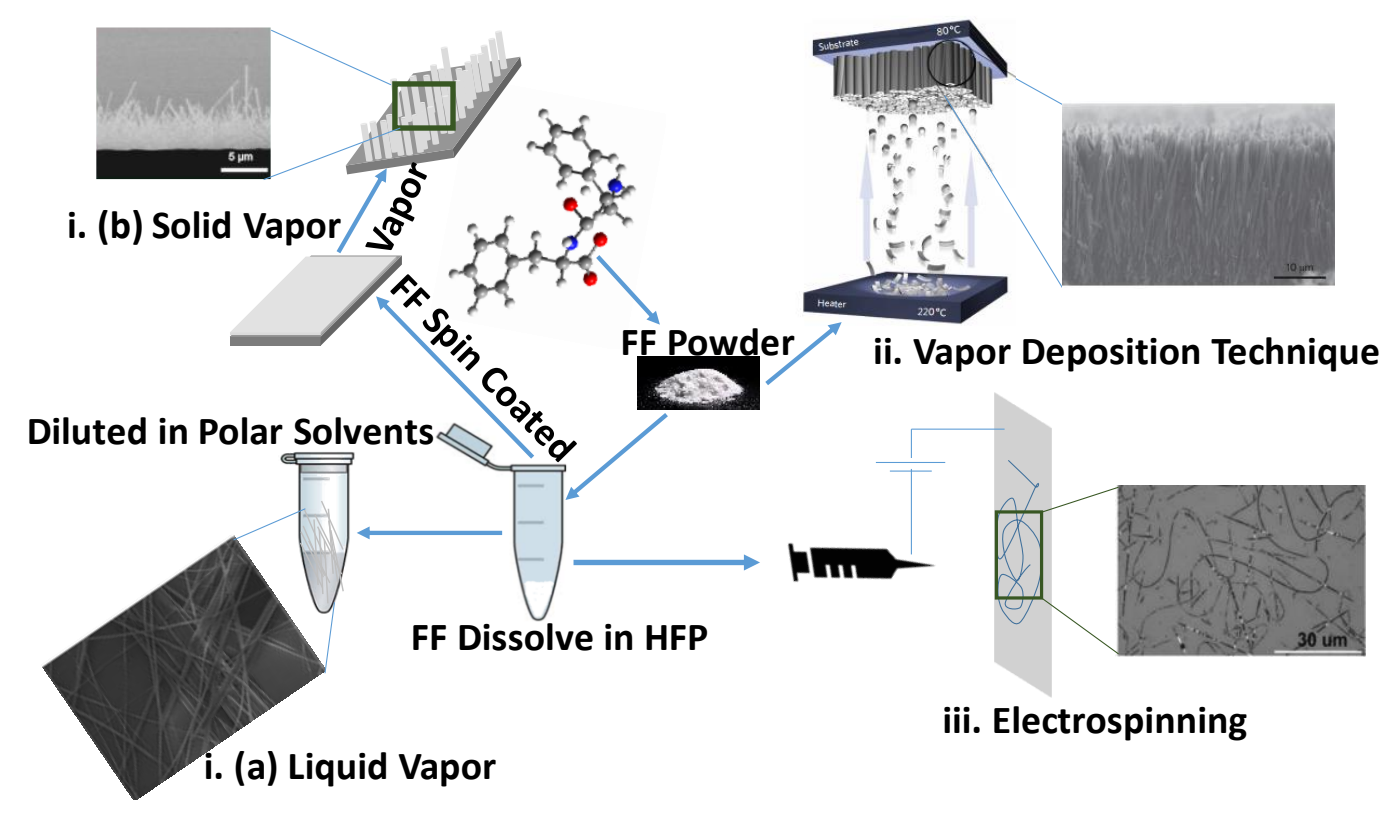

Figure 1.5. Demonstrate different synthesis approach of peptide nanostructures. ${ }^{6}$

FF-MNS demonstrate two types of crystal structure: hexagonal and orthorhombic. ${ }^{12}$ Self-assembly occurred in a water-rich environment results in the hexagonal space group $\mathrm{P} 6_{1}$, which is non-centrosymmetric. In contrast, self-assembly occurred under anhydrous conditions results in the orthorhombic phase. ${ }^{8} \mathrm{XRD}$ data in Figure 1.6 shows these two phases. The phase transformation in FF-MNS from the hexagonal to the orthorhombic form can be achieved by annealing at $150^{\circ} \mathrm{C}$ for an hour in air. The insets of the Figure show the orientation of the molecule in a unit cell of these crystal structures. The optical and electrical properties of FF-MNS also change for the two phases. 

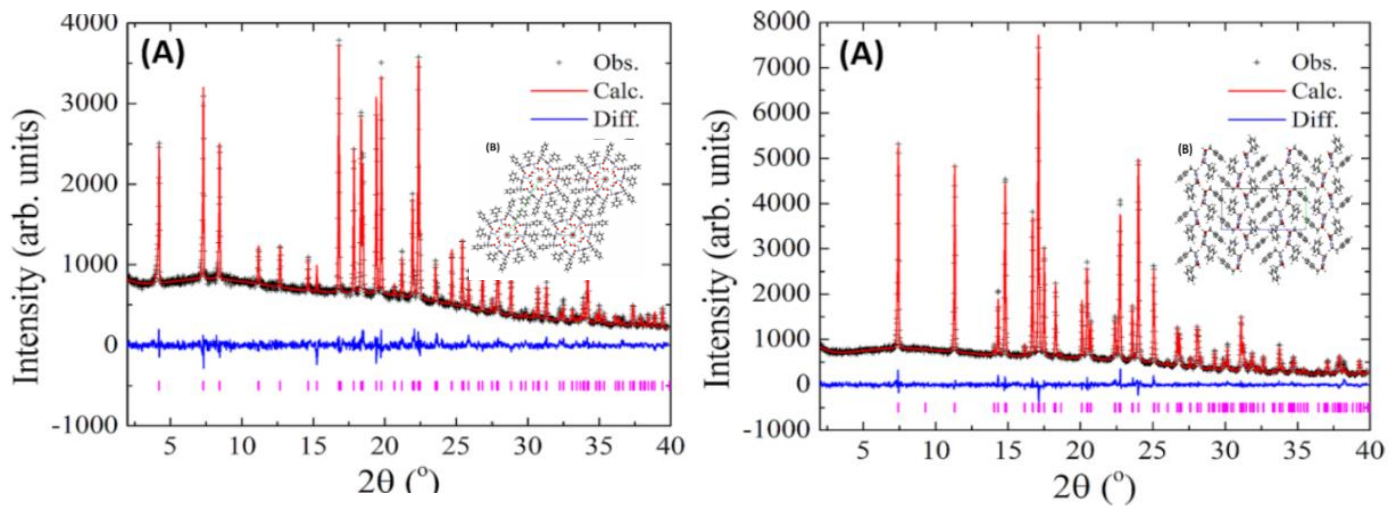

Figure 1.6. XRD data from FF-MNSs with Rietveld analysis in (a) hexagonal phase and (b) orthorhombic phase. Inserted images show the crystal structure packing obtained from XRD data. ${ }^{8}$ 


\section{Experimental and Theoretical Methodologies}

In this chapter, the characterization techniques used in our research will be discussed in detail. These include structural, optical, and magnetization properties. Structural characterization techniques including XRD, XPS, SEM, and transmission electron microscopy (TEM) are discussed. Optical characterizations were executed using absorption spectroscopy, photoluminescence spectroscopy (PL), Fourier transform infrared spectroscopy (FTIR), and Raman spectroscopy. We discuss the SHG setup, which was used to probe nonlinear properties. At the end of this chapter, SQUID magnetometer measurements, which was utilized to explore the magnetic properties, has been described.

\subsection{Structural Characterization Techniques}

\subsubsection{X-Ray Diffraction (XRD)}

$\mathrm{X}$-ray diffraction is a widely used nondestructive technique for the identification of a crystalline material. Each material possesses a unique diffraction pattern that make it possible to identify a material. This technique has the ability to determine bond length, bond angle, preferred orientation of the crystal, stress, strain, and particle size. The choice of sample could be bulk or thin film.

An X-ray diffractometer works on the phenomenon of diffraction of light. Monochromatic beam of X-rays interact with the sample and then detected by a detector to extract the structural information about the sample. The interatomic distance of materials is in the range of 0.15-0.4 nm. . The phenomenon of diffraction follows the Bragg's law. According to Bragg's law, constructive inference occurs if the following condition is satisfied:

$$
2 d \sin \theta=n \lambda,
$$


where $\mathrm{d}=$ interplanar spacing, $\mathrm{n}=$ order of diffraction, and $\lambda=$ wavelength of the source. For each constructive interference, there will be a peak in the intensity vs. $2 \theta$ plot, where $\theta$ is the incident angle of the X-ray.

The intensity of the peaks depends on several factors such as number of atoms, types of atoms, and atomic arrangement. The scattering intensity of the diffracted x-ray can be expressed as follows ${ }^{9}:$

$$
\mathrm{F}_{\mathrm{hkl}}=\sum_{j=1}^{m} N_{j} f_{j} \exp \left[2 \pi i\left(\mathrm{~h} x_{j}+k y_{j}+l z_{j}\right)\right.
$$

In equation (2.3), $\mathrm{F}_{\mathrm{hkl}}$ is called structure factor, which is the sum of scattering from all the atoms in the unit cell to form a diffraction peak originating from the (hkl) planes of atoms. $f_{j}$ quantifies the efficiency of X-ray scattering at any angle by the group of electrons in each atom. $\mathrm{Nj}$ is the fraction of every equivalent position that is occupied by atom $\mathrm{j}\left(x_{j}\right.$, $y_{j}, z_{j}$ ) represents the position coordinate for the atom $\mathrm{j}$.

The wavelength of the generated $\mathrm{x}$-rays depends on the choice of the material as anode. Several metals such as $\mathrm{Mo}, \mathrm{Cu}, \mathrm{Ni}, \mathrm{Fe}, \mathrm{Ag}$ are used as anode materials.

Three types of scattering process can occur when the X-ray interacts with a material. They are photoionization scattering, Compton scattering, and Thompson scattering. Photoionization scattering and Compton scattering are inelastic scattering, whereas Thompson scattering is elastic. Photoionization scattering occurs when light interacts with the material and transfer all its energy to excite an electron. This ejected electron around ionizes the neighboring atoms. A photon is not emitted in this process. Another type of interaction is Compton scattering where the light interacts with the materials; the energy 
and momentum are transferred to the bound electron by the photon, without releasing the electron from its bound state. Both Compton scattering and Thompson scattering are used for imaging tissue in human body. The last type of interaction is Thompson scattering which is used to elucidate structural information from a material. ${ }^{10}$ In this scattering process, an electron oscillates in accordance to a Hertz dipole at the frequency of the incoming electromagnetic beam and becomes a source of dipole radiation. The wavelength of the X-ray is conserved in this process.

The XRD measurements were performed at Missouri State University, Springfield. The Bruker D8 Discover X-ray diffractometer, shown in Figure 2.1, is used to obtain the XRD pattern from the samples. The XRD system comprises various components: a) X-ray source (rotating copper target) and incident beam optics (pinhole collimator), b) Eulerian Cradle (Goniometer: for accurate positioning of the sample), c) Laser/video alignment (X, Y, Z, phi, chi scans), and d) multi wire proportional area detector. EVA (evaluation) software was used to plot the preliminary XRD scans. To generate X-rays, a $40 \mathrm{KV}$ voltage and a $40 \mathrm{~mA}$ current were applied. The detector was moved to collect data from 20 to $80^{\circ}$ with a step size of $0.02^{\circ}$. Bruker XRD commndar software was used to collect the data.

Some of the work were conducted at UFABC, Brazil. In this system, X-ray data were collected using a STADI-P powder diffractometer (Stoe, Darmstadt, Germany) in transmission geometry by using $\mathrm{K} \alpha 1\left(\lambda_{\mathrm{Mo}}=0.7093 \AA\right.$ or $\left.\lambda_{\mathrm{Cu}}=1.54056 \AA\right)$ wavelengths emitted by Mo or $\mathrm{Cu}$ anode and selected by curved $\mathrm{Ge}(111)$ crystals. The samples were packed between two $0.014 \mathrm{~mm}$ acetate-cellulose foils, which were held spinning during data collection. The diffracted intensities were collected by silicon microstrip detectors, Mythen 1K (Dectris, Baden, Switzerland), in the range from $7.000^{\circ}$ to $70.945^{\circ}(\mathrm{MoK} \alpha 1)$, 
with $100 \mathrm{~s}$ of integration time at each $0.78^{\circ}$ for the $\mathrm{CFO}$ system alone and in the range from $3.000^{\circ}$ to $62.835^{\circ}(\mathrm{Cu} \mathrm{K \alpha} 1)$, with $100 \mathrm{~s}$ of integration time at each $1.05^{\circ}$ for the FF-CFO system.

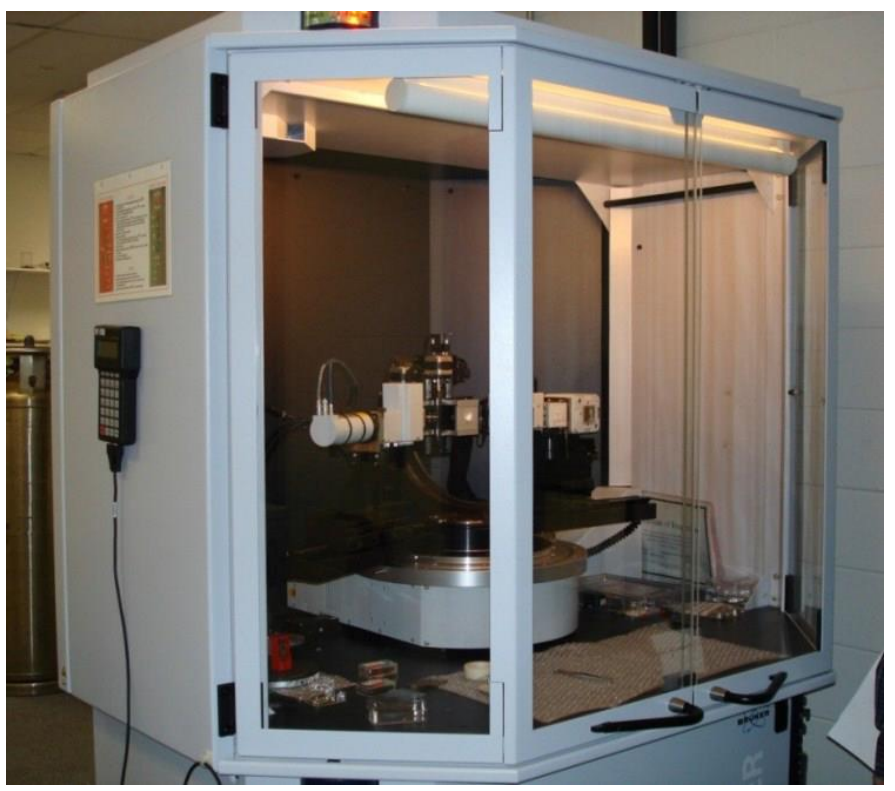

Figure 2.1. XRD measurement system at Missouri State University.

\subsubsection{X-Ray Photoelectron Spectroscopy (XPS)}

$\mathrm{X}$-Ray photoluminescence spectroscopy is a surface technique. It is widely used for bonding information, chemical, and electronic states of the elements on the surface of the material, identification of all elements except $\mathrm{H}$ and $\mathrm{He}$, depth profiling with matrix level concentrations, and thickness measurement of the oxide layer. It can collect information up to $10 \mathrm{~nm}$ in depth from the surface of the sample. A specimen placed in ultra-high vacuum is irradiated with photons of energy hv). Atoms on the surface emit electrons, called photoelectrons, after direct transfer of energy from the photons to its core-level's electron. ${ }^{11}$ Some of these emitted electrons overcome the binding energy and can be detected. Electromagnetic lens is used to focus the ejected electron from the surface onto 
the energy analyzer. The intensity of the emitted electrons is analyzed with respect to the kinetic energy. Figure 2.2 shows the working principle of an XPS measurement system.
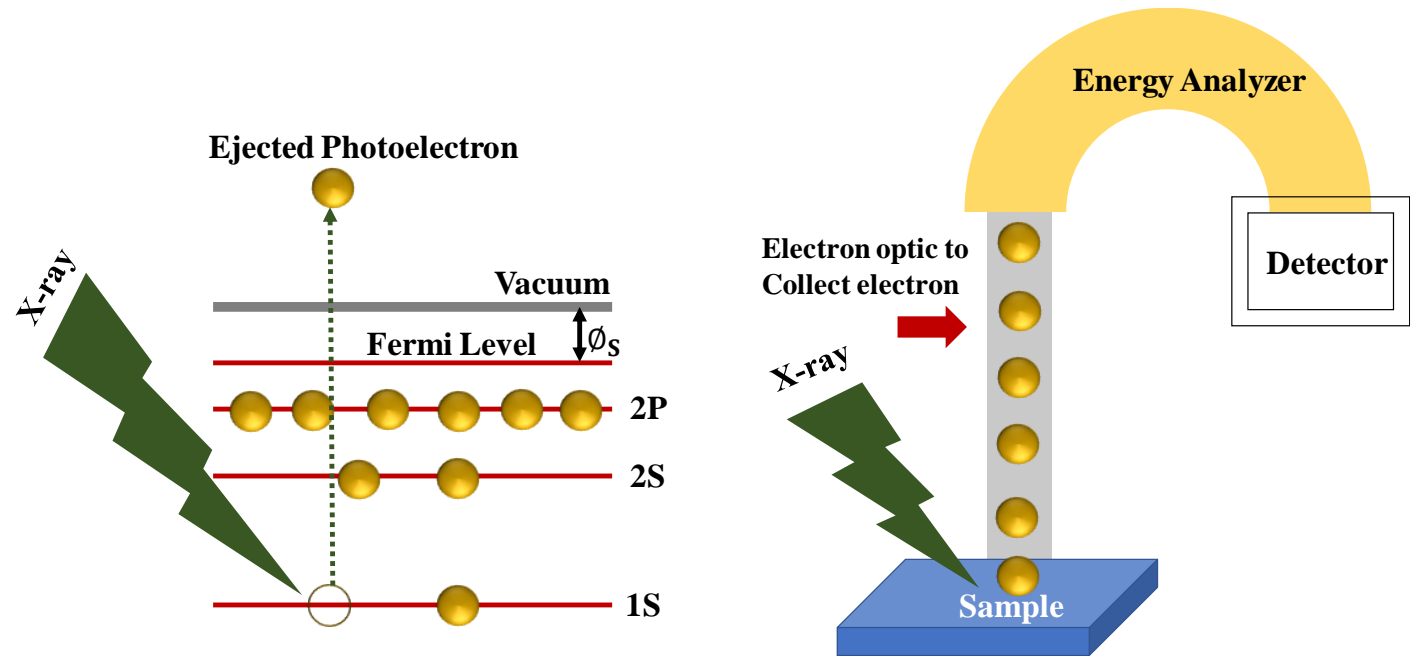

Figure 2.2. Illustrating the working principle of the XPS.

This measurement system has the following components: (i) X-ray source, (ii) electron optics, (iii) hemispherical energy analyzer, and (iv)detector connected with a computer. The XPS data were collected using a Thermo Scientific Alpha 110 hemispherical analyzer with pass energy of $25 \mathrm{eV}$. Twin crystal monochromatic $\mathrm{Al} \mathrm{K}_{\alpha}$ with a characteristic energy of $1486.6 \mathrm{eV}$ was used as a source of $\mathrm{X}$-ray. The $\mathrm{Ag} 3 \mathrm{~d}_{5 / 2}(\mathrm{FWHM}=0.36 \mathrm{eV})$ peak with a characteristic energy of $368.26 \mathrm{eV}$ was used for calibration of the source and analyzer. Casa XPS 2.3.16 software is used for data analysis and peak fitting of the XPS spectra. The carbon peak at $284.8 \mathrm{eV}$ was used for the calibration of the XPS data. Shirley background was used for the fitting of the high-resolution data. The experimental data were fitted with a combination of Lorentzian and Gaussian line profiles.

\subsubsection{Scanning Electron Microscopy (SEM)}

Scanning electron microscopy is a widely used imaging technique for exploiting the surface morphology, identifying chemical elements and their binding, and determining 
particle size. It works on the same principle as an optical microscope. An electron beam interacts with a sample and generates the information about the sample. The system is made up of two main components; the electronic console and the electron column. ${ }^{12}$ The electronic console consists of control knobs and switches that allow for the instrument adjustments such as accelerating voltage, focus, magnification, filament current, brightness, and contrast. The electron column consists of an electron gun, electromagnetic lens, sample chamber, high vacuum system, and a detector. The electron gun generates an electron beam using the electron gun; the beam is then directed and focused onto the sample through the electromagnetic lens. Several signals are generated from the interaction of the electron beam with the sample. Various detectors are used to detect different types of signal. The Figure 2.3 shows the configuration of a typical SEM.

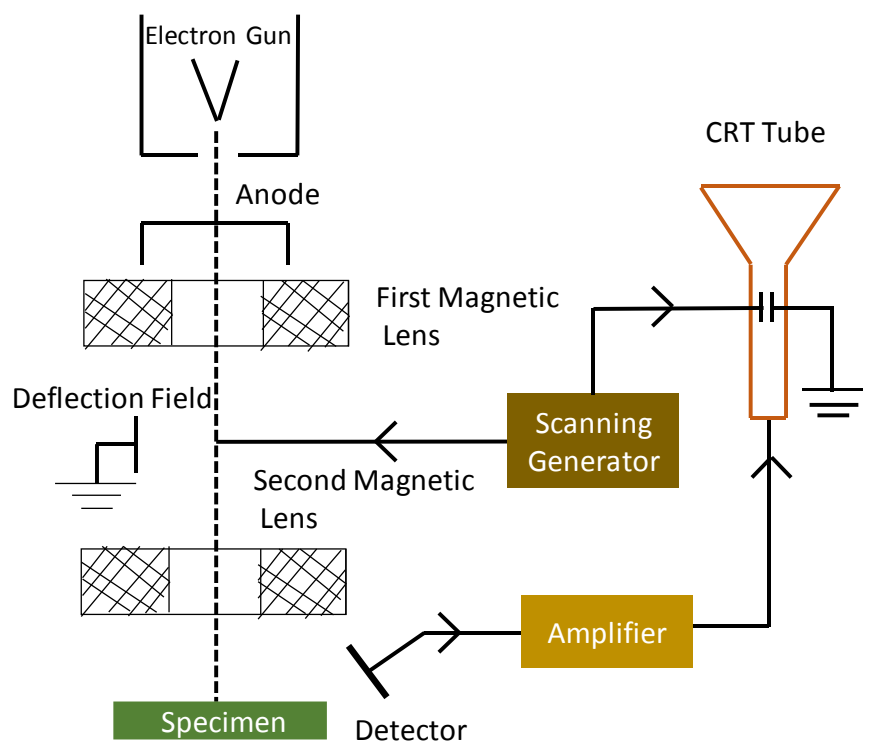

Figure 2.3. Configuration of a typical SEM.

For this research work, some of the data were collected at the University of Missouri Electron Microscopy Core facility using Quanta 600F Environmental SEM. Others were collected using FEI Quanta 200 S FESEM at Missouri State University. Scanning electron 
microscopy specimens were made more conductive by the application of thin $\mathrm{Pt}$ or $\mathrm{Au}$ coating, using an EMS575X sputter coating. The SEM was operated in high vacuum mode with an accelerating voltage of $5-10 \mathrm{KeV}$ and spot size of 3.

\subsection{Optical Characterization Techniques}

\subsubsection{Absorption Spectroscopy}

The UV-visible spectroscopy is a widely used technique for determining optical properties such as optical band gap and absorption coefficient. The measurements can be done on liquid or solid sample. UV-visible light is transmitted through a sample and by comparing the transmitted intensity to the incident intensity, the absorption coefficient may be deduced. The Beer-Lambert's law yields

$$
I(\lambda)=I_{0} \exp (-\alpha(\lambda) t)
$$

where $I_{0}=$ intensity of the light before passing through the sample, $I(\lambda)=$ intensity of the light after passing through the sample with thickness of $t$.

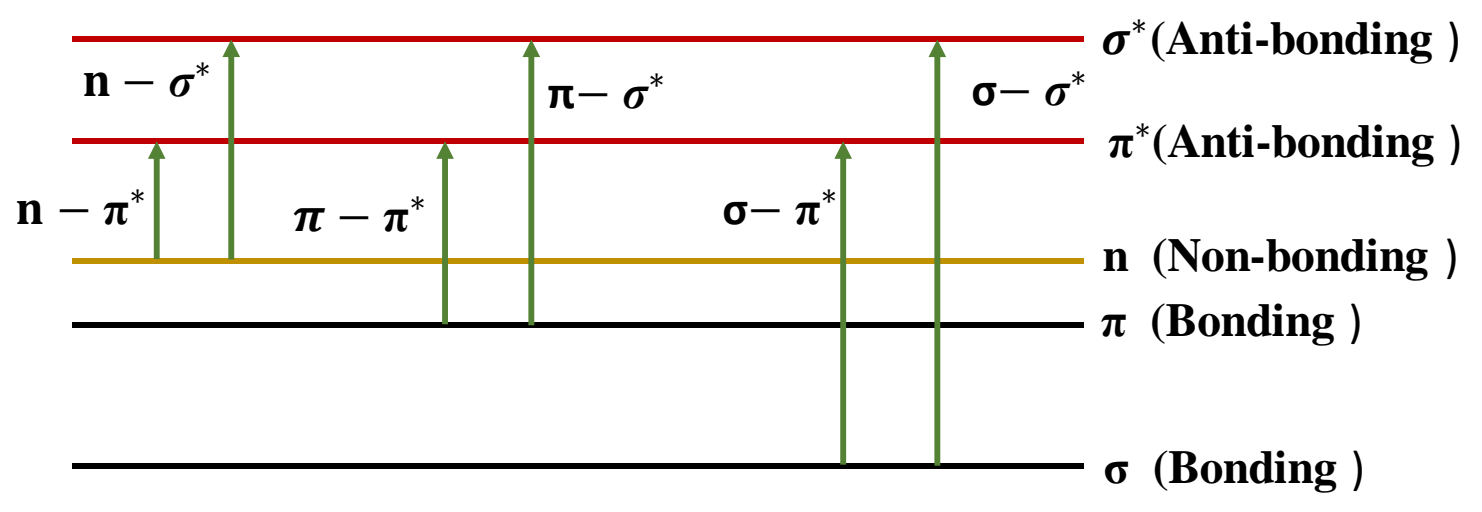

Figure 2.4. Electronic transitions in a molecule.

Absorption spectroscopy is used to probe the electronic transition. Figure 2.4 shows the electronic transitions in a molecule. ${ }^{13}$ Out of these transitions, $n-\pi^{*}$ and $\pi-\pi^{*}$ may be investigated with a wavelength range of $200-800 \mathrm{~nm}$. Rest of the transition need higher 
energy to occur. The $\pi-\pi^{*}$ gap is reduced with increasing $\pi$ conjugation in organic molecules. The vibrational and rotational energy levels cause broadening of the absorption peak.

The absorption spectra were measured using a Shimadzu UV-2401 PC. A glass slide was used as a reference sample. The light source is a halogen lamp for the visible region and a deuterium lamp for the ultra-violet region. The detector is a photomultiplier R- 928.

\subsubsection{Photoluminescence Spectroscopy}

Photoluminescence spectroscopy is another technique for measuring electronic structures of a material. Upon absorption of a photon, a neutral excited state, the singlet exciton, is created followed by both radiative (PL) and non-radiative recombination processes. In molecular materials, the singlet excitons are Frenkel-type, which are tightly bound with large binding energies $(\sim 1 \mathrm{eV}) .{ }^{14}$ Through a change in their spin multiplicity singlet excitons may populate the triplet exciton states, which have long lifetimes. Triplet generation can be probed by triplet-triplet $(\mathrm{T} 1-\mathrm{TN})$ absorption using modulation spectroscopy. The singlet excitons may further dissociate to form singly charged polarons or polaron pairs resulting in a polaronic absorption. The polarons are generated either via exciton dissociation (electric fields, defects, and electron or hole acceptors) or via direct creation (charge injection or doping mechanisms). In addition to the spin $1 / 2$ polarons, spinless bipolarons occur, which arise from gap states that are deeper than the polaron states. The population of the polaron and triplet states leads to a small depopulation of the ground state, giving rise to a bleaching of the absorption. 
(a)

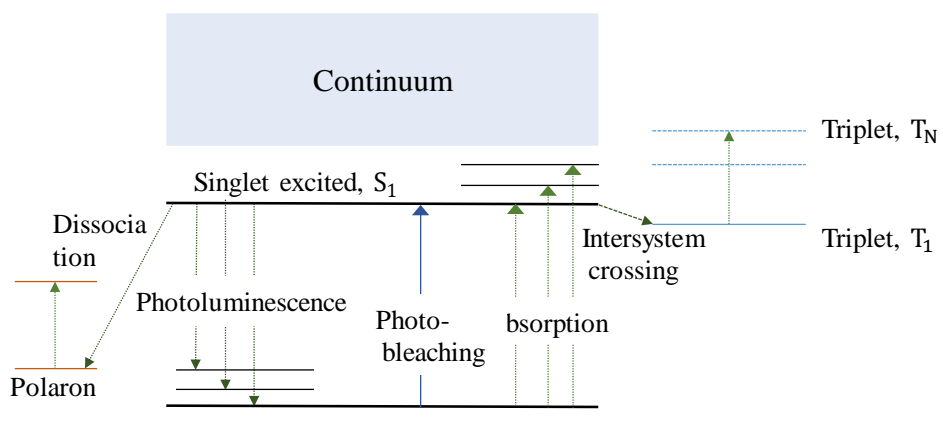

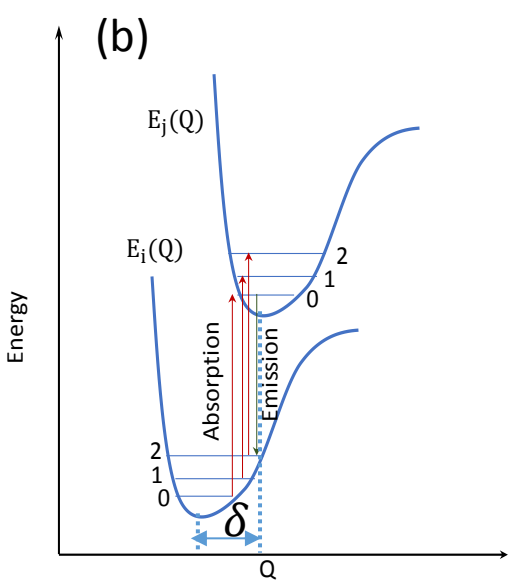

Figure 2.5. (a) A schematic diagram of energy levels in conjugated molecules and polymers. (b) Electronic ground and excited states in configuration space. ${ }^{14}$

The absorption and PL spectra of conjugated polymers and molecules are characterized by a well-resolved Franck Condon (FC) type progression of the vibronic sub-bands in addition to the main $\pi-\pi^{*}$ electronic transition. In the adiabatic picture, a vibronic progression in the electronic spectra implies that the ground and excited state equilibrium structures are displaced relative to one another in configuration space, as shown in Figure 2.5 (b). The absorption and the PL spectra typically show a Stokes shift, which is related to the displacement of the ground and excited states $(\delta)$.

Figure 2.6 represents the experimental set up, which we have used for this work. In our work, a He Cd laser with a wavelength of $325 \mathrm{~nm}$ has been used. Using mirrors, the laser light is focused onto the sample. A narrow band pass filter is used before focusing the light on the sample. The samples were kept inside a vacuum chamber. The focused PL spectra are collected with the help of an Ocean Optics, which is connected, to a computer. A bandpass filter, which allows the light above $400 \mathrm{~nm}$, is used before collecting the PL spectra. 


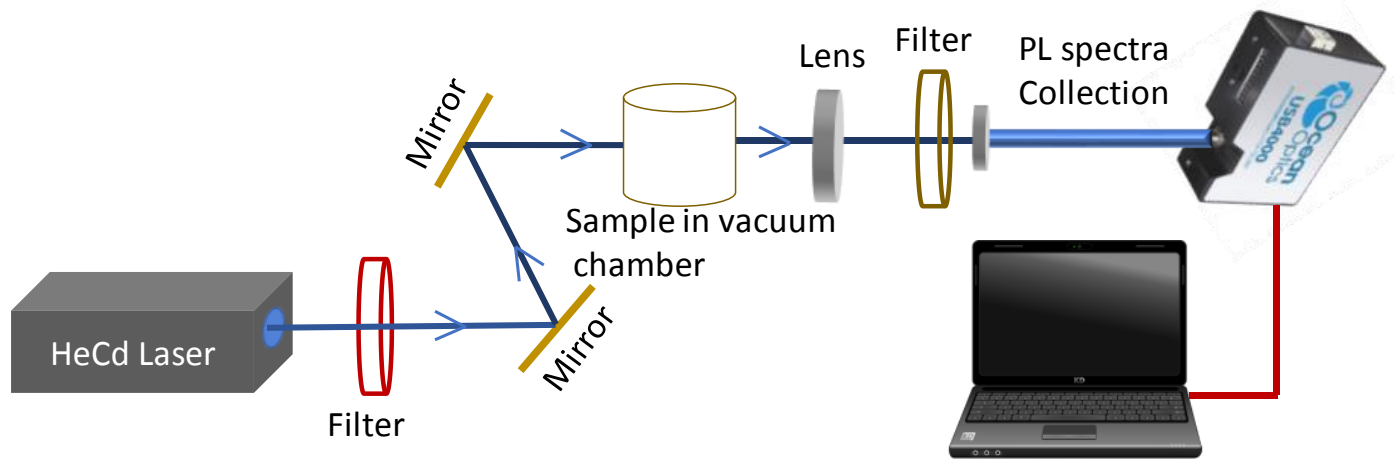

Figure 2.6. Schematic diagram of the experimental set up for photoluminescence measurement.

\subsubsection{Fourier Transform Infrared Spectroscopy (FTIR)}

FTIR provides information on the absorption or transmission of the infrared spectra for a wide range of wavelengths in a single measurement. In this spectroscopy, an interferogram is generated over a wide range of frequencies using the principle of a Michelson interferometer. The light from the source splits into two with the same intensity by the beam splitter. One part is directed towards a fixed mirror and the other part is directed towards a moving mirror. These mirrors are perpendicular to each other. The velocity of the moving mirror can be controlled. After reflection from these mirrors, the reflected waves recombine at the beam splitter through the phenomenon of interference as there is path difference between the two recombined reflected waves. This intensity vs. time wave is called "interferogram". Figure 2.7 shows the working principle of generating an interferogram. 


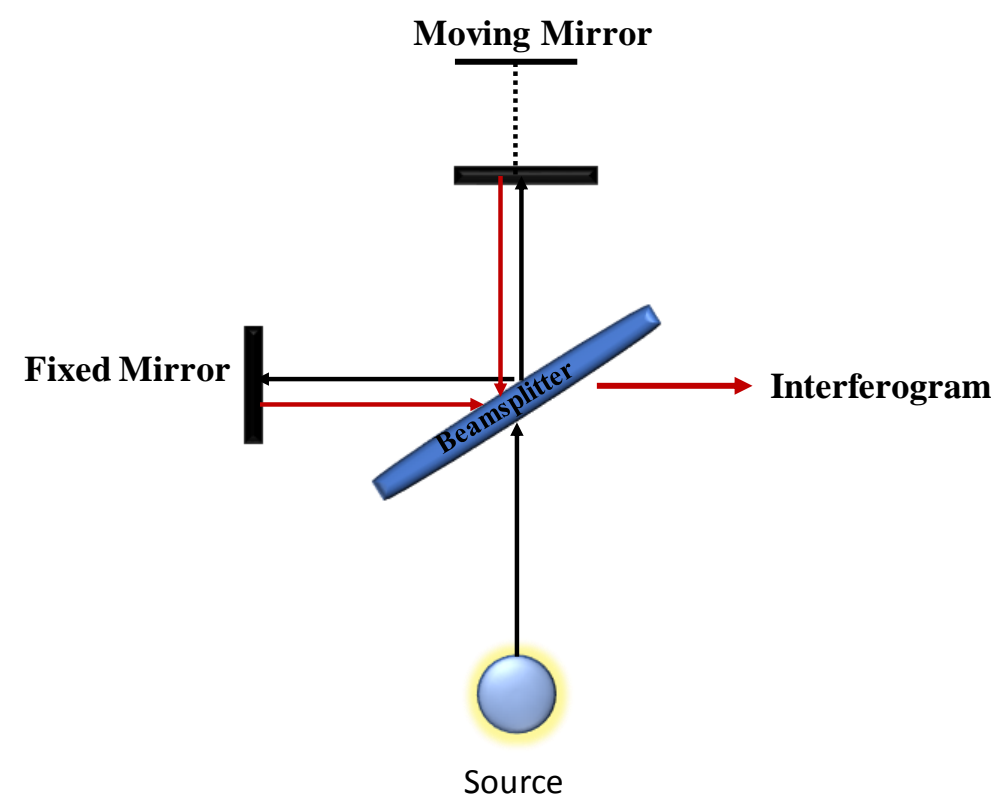

Figure 2.7. Schematic of an Interferogram.

The generated interferogram is passed through the sample and the absorption or transmission of this signal is recorded by the detector. This change in the intensity data with time give the information about the structure of the specimen. A Fourier transform is used to covert the intensity vs. time graph to an intensity vs. frequency graph. A laser light is superimposed to calibrate the instrument operation.

In my research work, the ATI Mattison Infinity 60AR FTIR was used for measurement of the FTIR spectra. It has two sources: visible or NIR (tungsten halogen) and midinfrared. A He-Ne laser is also included in the FTIR. The monochromatic wavelength of the He-Ne laser is used as a reference to calibrate the path difference. The reflection mode was used, as the samples used were not transparent.

\subsubsection{Raman Spectroscopy}

Raman spectroscopy, named after C. V. Raman, is a technique to the probe vibrational, rotational and other low-frequency modes in a system. It provides a structural fingerprint 
to identify molecules. Light matter interaction causes absorption, transmittance or scattering of the light by the matter.

When a photon interacts with a molecule, a vibration state may get excited to another virtual vibration state. This excited molecule returns to its ground state by re-emitting photons, and it might fall into another vibrational state or in the same state. A photon that induces a transition where the net result is a transition from the ground vibrational state to a higher vibrational state is said to have undergone a Stokes shift. Whereas a photon that induces a transition where the net result is a transition from a higher vibrational state to the ground state is said to have anti-Stokes shift. Figure 2.8 shows a schematic of the Rayleigh and Raman scattering process.

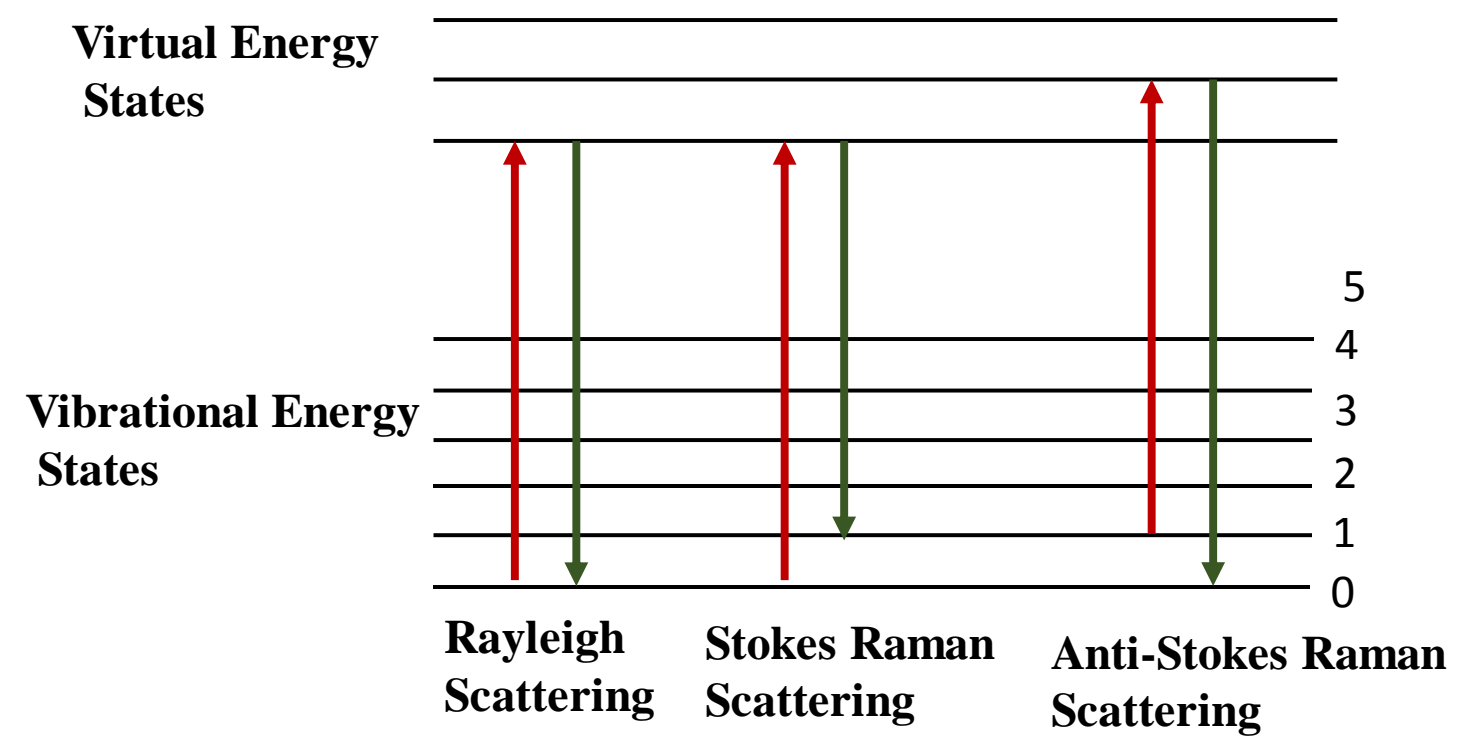

Figure 2.8. Energy level of a molecule showing different types of Raman signal.

The condition for a molecule to be Raman active is, at least, one of the components of the polarizability tensor must be non-zero. The total molecular polarizability of a polyatomic molecule is made of contributions from individual bond polarizabilities.

The dipole moment may be expressed as: ${ }^{15}$ 


$$
P=\alpha E,
$$

where $\mathrm{P}=$ dipole moment, $\alpha=$ polarizability, and $\mathrm{E}=$ external electric field. The applied electric field can be expressed as

$$
E=E_{0} \cos \left(\omega_{i} t\right)
$$

$\alpha$ can be expressed as the following as each vibration contributes to the polarizability. Only the first term of the Taylor expansion has been considered:

$$
\alpha=\alpha_{0}+\left(\frac{\partial \alpha}{\partial q}\right)_{0} q
$$

where $\alpha_{0}=$ permanent polarizability, $\mathrm{q}=$ normal coordinate which can be taken as the following if the vibration is small and can be considered oscillate harmonically then

$$
q=q_{0} \cos \left(\omega_{v i b} t\right)
$$

Combining equation (2.6) to (2.8), the dipole moment can be derived as

$$
\begin{gathered}
P=\mu_{0}+\alpha_{0} E_{0} \cos \left(\omega_{i} \mathrm{t}\right)+\left(\frac{\partial \alpha}{\partial q}\right)_{0} E_{0} q_{0} \cos \left(\omega_{i} t\right) \cos \left(\omega_{v i b} t\right) \\
=\mu_{0}+\alpha_{0} E_{0} \cos \left(\omega_{i} t\right)+\frac{1}{2}\left(\frac{\partial \alpha}{\partial q}\right)_{0} E_{0} q_{0}\left[\cos \left(\left(\omega_{i}+\omega_{v i b}\right) t\right)\right. \\
\left.+\cos \left(\left(\omega_{i}-\omega_{v i b}\right) t\right)\right]
\end{gathered}
$$

where $\mu_{0}=$ permanent dipole moment.

The second term represents the Rayleigh scattering without changing frequency, and the third term represents the Raman scattering where there is a change in the frequency of the re-radiation by the light irradiated molecule. From this expression, it is clear, if $\left(\frac{\partial \alpha}{\partial q}\right)_{0}=0$ then we don't observe Raman effect. This implies that polarizability must change with respect to the vibration for the observation of the Raman effect. The dipole moment of the 
molecule should change in order to be Infrared active. This causes some of the vibration modes to be either Raman active or IR active not both at the same time in systems with a mirror plane symmetry. There are mainly two types of vibration modes: stretching vibration and bending vibration. Stretching vibration can be classified into symmetric stretching and asymmetric stretching vibration. A bending vibration is categorized as scissoring, rocking, wagging, and twisting. The number of vibration modes is $3 \mathrm{~N}-5$ for linear molecule and $3 \mathrm{~N}-6$ for a non-linear molecule, where $\mathrm{N}=$ number of atoms in the molecule. For $\mathrm{CO}_{2}$ molecules, there are 3 atoms; so number of vibration modes is $3 \times 3$ $5=4$. These vibration modes are shown below.

(a)

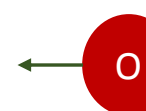

0

(c)

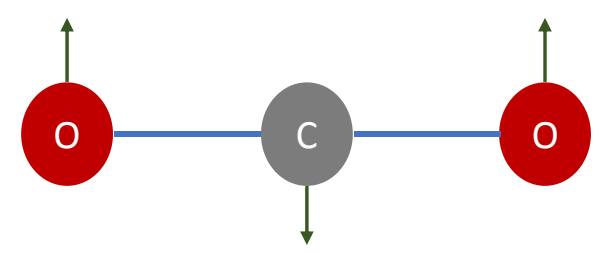

(b)

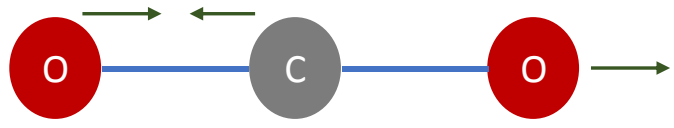

(d)

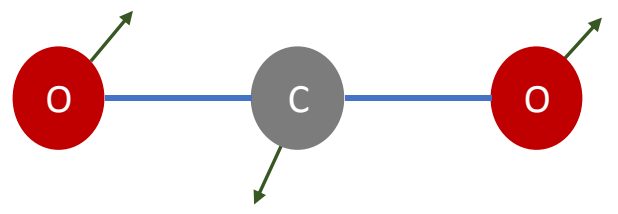

Figure 2.9. (a) Symmetric stretching vibration, (b) Asymmetric stretching vibration, (c) Bending vibration, and (d) Bending vibration.

Figure 2.9 (a) and (b) show symmetric and asymmetric stretching respectively. For a symmetric stretching, the change in the total dipole moment is zero. Therefore, this mode is not IR active mode. However, the change of the polarizability is not zero, which makes this vibration mode as Raman active. Figure 2.9 (b), which represents the asymmetric vibration mode, the total dipole moment changes but the total bond polarizability with 
respect to this vibration mode does not change. Therefore, this vibration mode is IR active but not Raman active.

Figure 2.10 shows a schematic diagram of a micro-Raman setup. It consists of (1) an excitation source, generally a continuous wave (CW) laser, (2) collimated optics, (3) collection optics to collect the Raman scattered photons, (4) a monochromator to separate the Raman signal into its constituent wavelengths, (5) a detector to detect the scattered photons, and (6) a computer system to store and display the spectra. The Raman spectra were collected by an Invia Renishaw spectrometer attached to a microscope with a 50x lens. The system is equipped with two lasers: a $785 \mathrm{~nm}$ diode laser and a $514 \mathrm{~nm}$ argon ion laser.

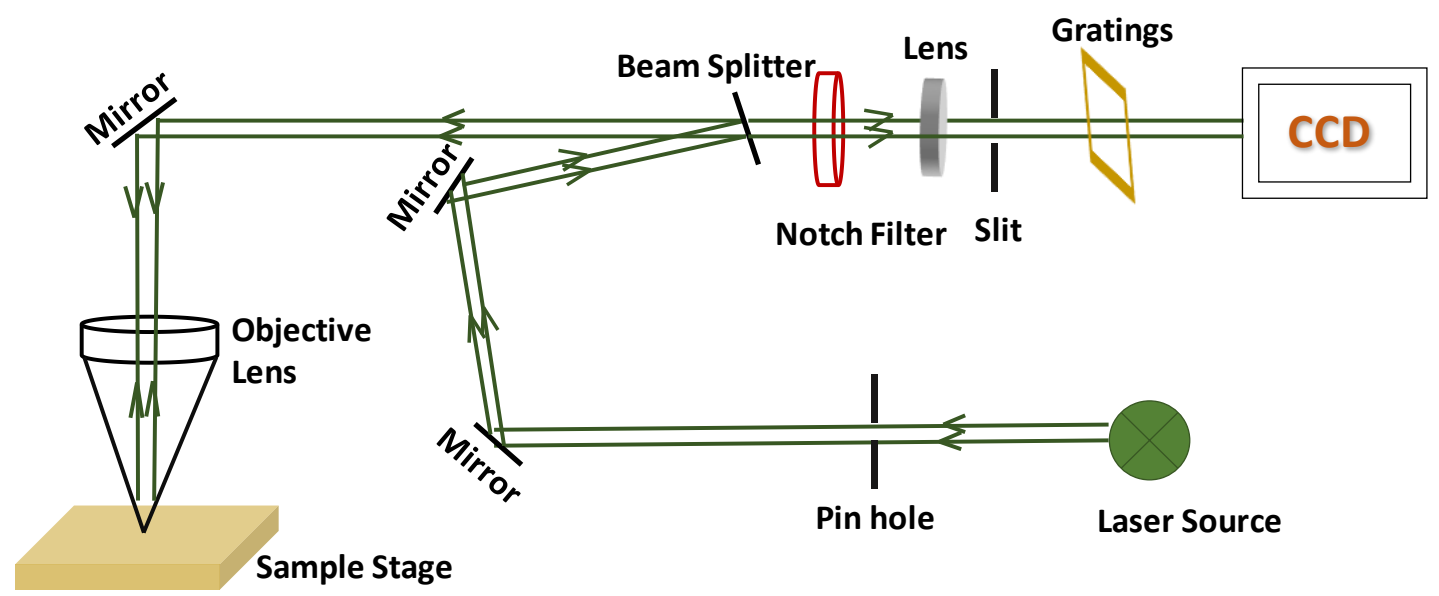

Figure 2.10. Schematic representation of Raman Microscope.

\subsection{Second Harmonic Generation Experimental Setup}

Second harmonic generation (SHG) constitutes the process of doubling the frequency of the fundamental incident light. The SHG interaction of the dominant electric-dipoles involves three photons; two input photons of frequency $\omega$, and one output photon of frequency $2 \omega$, and occurs only in materials that do not possess inversion symmetry. ${ }^{16} \mathrm{SHG}$ is a powerful technique for the structural studies of systems that are non-centrosymmetric. 
In general, a large number of bioinspired materials have a non-centrosymmetric crystal structure $^{17}$; self-assembly of these materials resulting in tubular structures creates appropriate experimental conditions for observing SHG. ${ }^{18-19}$

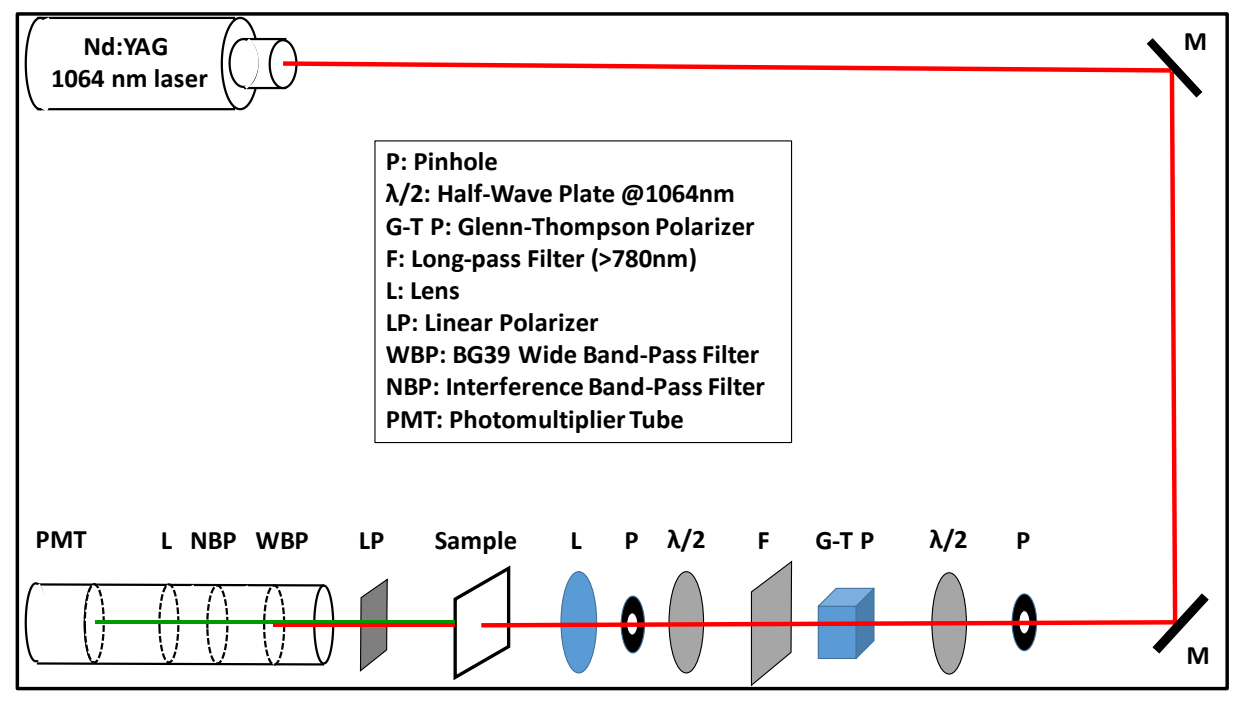

Figure 2.11. SHG experimental setup.

In general, the polarization $\mathrm{P}$ in presence of an electric field, $\mathrm{E}$, may be expressed as:

$$
\vec{P}(t)=\overrightarrow{P_{0}}+\varepsilon_{0} \chi_{e} \vec{E}(t)+\chi^{(2)} \vec{E}(t)^{2}+\chi^{(3)} \vec{E}(t)^{3}+\cdots
$$

Where, $P_{0}$ is the time independent constant polarization, and $\chi$ is the susceptibility. The $\vec{E}(t)^{2}$ is responsible for the SHG process. So the polarization in the SHG process may be expressed as:

$$
P^{2 \omega}=d\left(E^{\omega}\right)^{2}
$$

Conventionally, instead of $\chi^{(2)}$, the notation $\mathrm{d}$ is used. The tensorial form is given by

$$
P_{i}^{\omega_{3}}=\sum_{j, k} d_{i j k} E_{j}^{\omega_{1}} E_{k}^{\omega_{2}} .
$$

In case of SHG, $\omega_{1}=\omega_{2}$ and $\omega_{3}=2 \omega$. So,

$$
\begin{array}{r}
P_{1}=d_{111} E_{1} E_{1}+d_{112} E_{1} E_{2}+d_{113} E_{1} E_{3}+d_{121} E_{2} E_{1}+d_{122} E_{2} E_{2} \\
+d_{123} E_{2} E_{3}+d_{131} E_{3} E_{1}+d_{132} E_{3} E_{2}+d_{133} E_{3} E_{3}
\end{array}
$$


Since $E_{j} E_{k}=E_{k} E_{j}$ and $d_{i j k}=d_{i k j}$, equation (2.14) can be written as:

$$
\begin{aligned}
& P_{1}=d_{111} E_{1} E_{1}+d_{122} E_{2} E_{2}+d_{133} E_{3} E_{3}+2 d_{112} E_{1} E_{2}+ \\
& 2 d_{113} E_{1} E_{3}+2 d_{123} E_{2} E_{3} .
\end{aligned}
$$

An abbreviated notation is typically used, where subscripts 23 or 32 are substituted by subscript 4,31 or 13 by 5 , and 12 or 21 by 6 . So,

$$
\begin{gathered}
P_{1}=d_{11} E_{1}{ }^{2}+d_{12} E_{2}{ }^{2}+d_{13} E_{3}{ }^{2}+2 d_{16} E_{1} E_{2}+ \\
2 d_{15} E_{1} E_{3}+2 d_{14} E_{2} E_{3} .
\end{gathered}
$$

We can write similar equations for $P_{2}$ and $P_{3}$ as well. Thus, the complete second harmonic generation process is given as:

$$
\left(\begin{array}{l}
P_{1}(2 \omega) \\
P_{2}(2 \omega) \\
P_{3}(2 \omega)
\end{array}\right)=\left(\begin{array}{llllll}
d_{11} & d_{12} & d_{13} & d_{14} & d_{15} & d_{16} \\
d_{21} & d_{22} & d_{23} & d_{24} & d_{25} & d_{26} \\
d_{31} & d_{32} & d_{33} & d_{34} & d_{35} & d_{36}
\end{array}\right)\left(\begin{array}{c}
E_{1}^{2}(\omega) \\
E_{2}^{2}(\omega) \\
E_{3}^{2}(\omega) \\
2 E_{2}(\omega) E_{3}(\omega) \\
2 E_{1}(\omega) E_{3}(\omega) \\
2 E_{1}(\omega) E_{2}(\omega)
\end{array}\right) .
$$

Our SHG setup uses a Q-switched YAG:Nd ${ }^{3+}$ laser at a wavelength of $1064 \mathrm{~nm}$ with a spot size of about $100 \mu \mathrm{m}$. It was configured in the transmission geometry as shown in Figure 2.11. A laser pulse duration of $20 \mathrm{~ns}$ and a repetition rate of $1 \mathrm{kHz}$ were used for the measurements. The SHG signal was collected with a photomultiplier tube detector and measured with a box-car.

\subsection{Magnetic Characterization Technique: SQUID Magnetometer}

Superconducting quantum interference device (SQUID) magnetometer is used to measure the magnetic properties of materials. Temperature dependent and field dependent magnetization can be investigated. This magnetometer is highly sensitive with an ability to measure magnetization as small as the order of $10^{-15}$ Tesla. The ability to measure change in magnetic field associated with one flux quantum $\emptyset_{0}$, where $\emptyset_{0}=\frac{2 \pi \hbar}{2 e} \approx 2.06 \times 10^{-15}$ 
tesla.m ${ }^{2}$ explains the sensitivity of the SQUID device. The main components of a SQUID magnetometer are a superconducting magnet, a superconducting detection coil, a SQUID (consisting Josephson junction and superconductor), a superconducting magnetic shield, a heating and cooling system, and a thermal isolation system. ${ }^{20}$

Figure $2.12^{21}$ shows how a SQUID magnetometer works. The superconducting magnet is made of $\mathrm{Nb}$ wire, which produces a magnetic field $\mathrm{B}$ by sending a current. The sample is moved through this external magnetic field inside a detection coil or pickup coil using a hydraulic system in order to avoid mechanical vibrations. The magnetic moment of the sample induces a magnetic flux change in the pick-up coil which is connected to the superconducting loop with a parallel Josephson junction. A Josephson junction consists of two superconductors separated by a thin insulating layer that helps electrons in quantum tunneling from one layer to another. The magnetic flux from the moving sample interrupts the superconducting loop. If the biasing current is constant to the SQUID device, then the measured voltage oscillates with the change in phase at the two junctions depending on the flux change arising from the movement of the magnetic sample. The SQUID device and the pickup coil are insulated from the external magnetic field. A small heater, made of Allan-Bradley resistor, is attached to the sample holder for temperature dependent magnetization measurement. Thermocouple, attached at the top of the sample holder, is used to record the temperature of the sample.

Field dependent magnetization measurements, $M(H, T)$, were carried out using the SQUID magnetometer (Model MPMS-5, Quantum Design Inc.) available at Missouri State University. This system has the ability to vary the magnetic field from $-5 \mathrm{~T}$ to $5 \mathrm{~T}$ and the temperature from $5 \mathrm{~K}$ to $300 \mathrm{~K}$. 


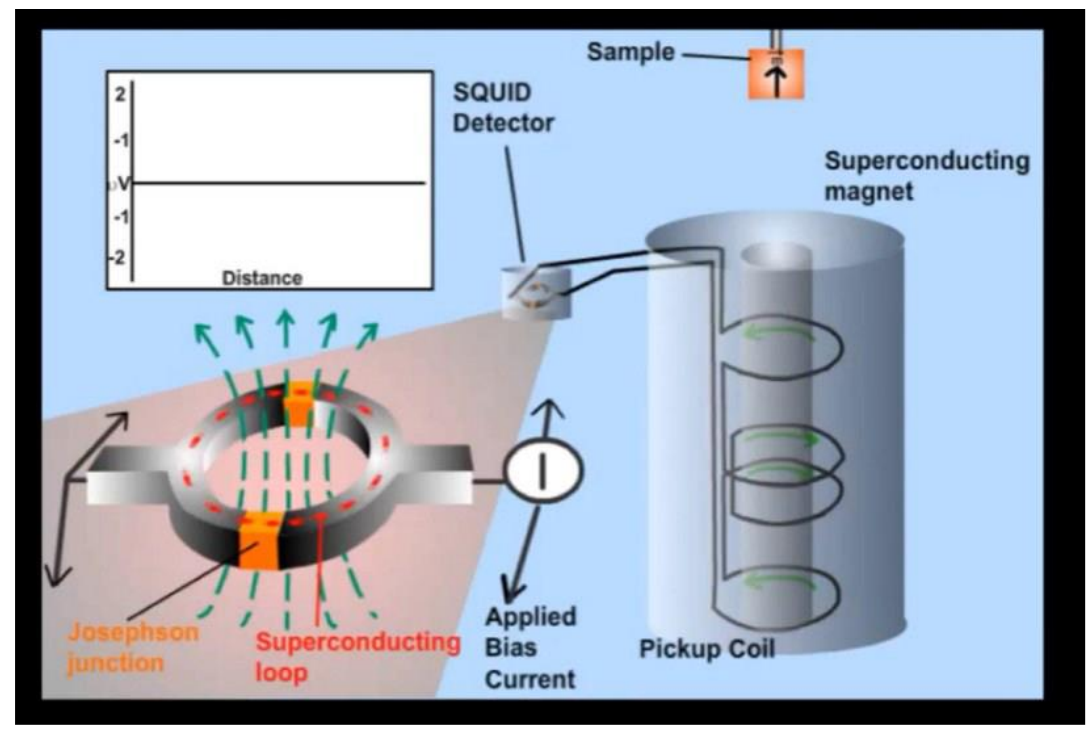

Figure 2.12. Schematic diagram of a SQUID magnetometer showing working principle of the magnetometer. ${ }^{21}$

\subsection{Density Functional Theory (DFT)}

DFT is widely used quantum mechanical approach to investigate the structural, magnetic, and electronics properties of molecules, materials, and defects. The quantum mechanical wavefunction contains, in principle, all the information about a given system. The wavefunction of a system can be determined by solving the Schrödinger equation. The time independent Schrödinger equation is given by

$$
\mathrm{H} \psi=\mathrm{E}_{0} \psi
$$

Where $\mathrm{H}$ is the Hamiltonian for the system, $E_{0}$ is the ground state energy of that system and $\psi$ is the wavefunction. The Hamiltonian of a $\mathrm{N}$ electron system can be expressed as:

$$
\begin{aligned}
H=\frac{\hbar^{2}}{2 m} \sum_{i} \nabla_{i}^{2} & +\frac{1}{2} \sum_{i \neq j} \frac{e^{2}}{\left|r_{i}-r_{j}\right|}-\sum_{i} \sum_{I} \frac{Z_{I} e^{2}}{\left|r_{i}-r_{I}\right|} \\
& +\frac{\hbar^{2}}{2} \sum_{I} \frac{\nabla_{I}^{2}}{M_{I}}+\frac{1}{2} \sum_{I \neq J} \frac{Z_{J} Z_{I} e^{2}}{\left|R_{J}-R_{I}\right|}
\end{aligned}
$$


where $\mathrm{m}$ is the electron mass, $\mathrm{M}$ is the nuclear mass, and $\mathrm{Z}$ is the atomic number of the element.. The first term and the second term represent the kinetic and the Coulombic repulsive energy between two electrons, respectively. The third and the fourth terms represent the Coulomb potential energy between the electron and the nucleus and the kinetic energy of the nucleus respectively. The fifth term represents the Coulomb potential energy between the nuclei. The fourth term is negligible as the mass of the nucleus is huge compared to the electron. So, the position of the nucleus is almost fixed. The fifth term is a constant. Using the Born-Oppenheimer approximation, equation (2.19) can be simplified as:

$$
H=\frac{\hbar^{2}}{2 m} \sum_{i} \nabla_{i}^{2}+\frac{1}{2} \sum_{i \neq j} \frac{e^{2}}{\left|r_{i}-r_{j}\right|}-\sum_{i} \sum_{I} \frac{Z_{I} e^{2}}{\left|r_{i}-r_{I}\right|}+\text { constant. }
$$

For large values of $\mathrm{N}$, solving the Schrödinger equation (2.18) is not possible. Evidently, we must involve some approximations to make the problem soluble. DFT method is based on the functional of electron density, which is a function of space coordinate $(\mathrm{x}, \mathrm{y}, \mathrm{z})$. Hohenberg and Kohn proposed that the ground state electron probability density should be enough to deduce the electronic properties of the system and it is unique and corresponds uniquely to an external potential. The single-particle density of the ground state of $\mathrm{N}$ interacting electrons at position $r$ can be written as:

$$
\mathrm{n}_{0}(\overrightarrow{\mathrm{r}})=\mathrm{N} \sum_{\sigma} \int \mathrm{d} \overrightarrow{\mathrm{x}_{2}} \cdots \int \mathrm{d} \overrightarrow{\mathrm{x}_{\mathrm{N}}} \mid \psi_{0}\left(\overrightarrow{\mathrm{r}} \cdot \sigma \cdot \overrightarrow{\mathrm{x}_{2}} \cdot \overrightarrow{\mathrm{x}_{\mathrm{N}}}\right\rfloor^{2}
$$

where $\psi_{0}$ is the ground-state wavefunction of the system at position $\boldsymbol{r}$, and $\sigma$ is spin.

In DFT calculation, the Kohn-Sham wavefunction is determined by imagining that there are non-interacting electrons whose density is exactly identical to the electron density of the real physical system. The calculation begins by providing a simple guess for the 
ground state density in the form of a basis set. Simple approximations of the dependence of energy on the density, known as functionals, can then be applied to determine the potential required to create this noninteracting electron density. Solving the Kohn-Sham Hamiltonian for this potential provides a refined density. Once this iterative approach has converged to a final density, the problem has been solved. To calculate the ground state energy of the actual interacting system, the final energy functional must take into account the exchange and correlation energy. The exchange energy considers the spin repulsive force between two wave functions of same spin. The correlation energy is the remaining unknown portion of the energy.

The total energy of $\mathrm{N}$ interacting particles can be written as,

$$
E_{T o t}[n]=T_{n i}[n]+E_{C}[n]+E_{x c}[n]+V_{0}[n] .
$$

where $T_{n i}=$ non-interacting kinetic energy, $E_{C}=$ Coulomb energy, and $E_{x c}=$ exchangecorrelation (xc) energy functional, and $V_{0}=$ external potential energy.

In this thesis, theoretical calculations such as optimized structure and vibrational spectra of small molecules were obtained using DFT and the B3LYP ( Becke's three parameter hybrid) functional in Gaussian 09 software. ${ }^{22}$ Basis set were $6-31+G(d, p)$ and $6-311++\mathrm{G}(2 \mathrm{~d}, 2 \mathrm{p})$ for organic molecule or biomolecule. The LANL2DZ basis $\operatorname{set}^{23}$ was used for the $\mathrm{Ag}$ and $\mathrm{Au}$ atom.

The Raman spectrum calculations are performed in two steps; first the force constant matrix is evaluated and then the eigenvalue equation is solved to obtain the eigenvalues and eigenvectors. ${ }^{24}$ The Raman scattering activities are proportional to the polarizability derivatives taken with respect to the cartesian coordinates. For a system of $\mathrm{N}$ atoms interacting via harmonic forces, the normal mode frequencies $\omega_{\mathrm{f}}$ and amplitudes $\chi(\mathrm{f})$ of 
mode $\mathrm{f}$, where $\mathrm{f}=1, \ldots, 3 \mathrm{~N}$ labels the normal modes, are determined by a $3 \mathrm{~N} \times 3 \mathrm{~N}$ matrix eigenvalue equation:

$$
\left(\varnothing-\omega_{\mathrm{f}}^{2} M\right) \chi(\mathrm{f})=0,
$$

where $\varnothing$ is the harmonic force constant matrix and $\mathrm{M}$ is the mass matrix. Both the force constant and mass matrices are symmetric. The intensities of the Raman peaks are theoretically obtained from the first derivatives of the polarizability with respect to the nuclear coordinates as the Raman effect depends on the polarizability derivative. The Raman scattering cross section is given by:

$$
\frac{\mathrm{d} \sigma}{\mathrm{d} \Omega}=\frac{\omega_{\mathrm{l}} \omega_{\mathrm{s}}{ }^{3}}{\mathrm{c}^{4}} \frac{\hbar}{2 \omega_{\mathrm{f}}}\left[\left\langle\mathrm{n}\left(\omega_{\mathrm{f}}\right)\right\rangle+1\right]\left\lfloor\alpha_{\mathrm{f}}\right\rfloor^{2} \mathrm{~L} .
$$

Where, $\mathrm{n}\left(\omega_{\mathrm{f}}\right)$ is the mean occupation number:

$$
\left\langle\mathrm{n}\left(\omega_{\mathrm{f}}\right)\right\rangle=\left[\exp \left(\beta \hbar \omega_{\mathrm{f}}\right)-1\right]^{-1}
$$

Here, $\omega_{\mathrm{f}}$ is the Raman shift frequency (in $\mathrm{rad} / \mathrm{s}$ ), $\omega_{\mathrm{l}}$ is the incident excitation frequency (in $\mathrm{rad} / \mathrm{s}$ ), $\omega_{\mathrm{s}}$ is the scattered frequency (in rad/s), $\left\lfloor\alpha_{\mathrm{f}}\right\rfloor^{2}$ is the Raman activity (in $\AA^{4} / \mathrm{amu}$,), and $\mathrm{L}$ is the local field correction.

To obtain the individual Raman scattering cross section (in units of $\mathrm{cm}^{2} / \mathrm{sr}$ ), the Raman activities obtained from the calculations were divided by the mode frequencies and multiplied by the thermal average occupation number at $300 \mathrm{~K}$ with the incident laser line as 785 or $514 \mathrm{~nm}$ to match the experimental conditions. ${ }^{25}$ 


\section{Solvent Driven Structural Changes in Self-Assembled FF-MNS}

\section{Nanostructures}

Contribution of different solvents with different dipole moment in FF-MNS formation have been explored in this chapter. DI water, DMSO, PC and toluene were used as solvent. We use DFT calculations to understand the underlying solvent driving mechanism in the formation of FF-MNS nanostructures. SEM, Raman scattering data and XRD data confirm the change in the crystal structures with varying solvents. These experimental results coincide with the theoretical prediction.

\subsection{Introduction}

Self-assembly process, which is the self-organization of molecules into long ordered structures under various non-covalent interactions, is observed in many biological complex systems such as DNA double helix, membrane, and protein folding. Many physiological functions depend on the self-assembly process and therefore, malfunctional self-assembly of a biomolecule causes disease. For example, protein fibril formation is the root cause of Alzheimer's disease. Controlling the self-assembly process of a biomolecule into highly ordered nanostructures is an interesting topic in materials science and supramolecular chemistry. The self-assembly process in FF offers versatile structures such as nanotube, nanovesicle, micelles bilayers, amyloid fibril, tapes and ribbon. These varying structures allow the usage of FF self-assembled architectures in wave guide (hollow tube), and drug delivery (nanosphere). Solvent, temperature, or $\mathrm{pH}$ can change the self-assembly process hence the nanostructures. Peptide self-assembly is a spontaneous thermodynamic and kinetic driven process, based on the synergistic effect of various intermolecular noncovalent interaction, including hydrogen bonding, $\pi-\pi$ stacking, electrostatic, 
hydrophobic, and Van-der Waals interaction. Solvent which is one of the kinetic parameter, modulate these interactions. ${ }^{26}$ Polarity and hydrogen bonding ability of the solvents directly influence local interaction of the self-assembly of peptide nanostructures on the molecular level..$^{27}$

Several studies show the impact of solvent on the self-assembly process of peptide nanostructures. Huang et al proposed that hydrogen-bond acceptor/ hydrogen-bond donor (HBD/HBA) ability and surface tension might have a major contribution in controlling the self- assembly process. ${ }^{27}$ Wang et al concluded that the directional hydrogen bonding promotes a long-range-ordered arrangement of $\mathrm{FF}$ molecules, preferentially along one dimension to form nanofibers or nanobelts. ${ }^{28}$ They have also discussed the influence of thermodynamic and kinetic factors on the structural assembly and regulation based on different types of peptide building blocks. ${ }^{26}$

In this chapter, we have studied the effect of solvent in the self-assembly process. We were able to highlight the effect of the solvents in changing the structure of the basic building of FF-MNS and correlate these changes with final nanostructures. For the first time, we show the change in lattice parameters with varying solvents. Electrostatic interaction, solvent-bridged hydrogen bond, and $\pi-\pi$ interaction are discussed in this chapter. Polarity, dipole moment, and hydrogen bonding capability of the solvents are important parameters to be considered for the self-assembly process. Figure 3.1 shows the molecular structure of the solvents used in the synthesis of FF-MNS. Table 3.1 lists the reported value of dielectric constant (polarity) and dipole moment. Toluene is a nonpolar aromatic hydrocarbon; water is a highly polar protic molecule with a strong hydrogen bonding capability; DMSO and PC are polar aprotic solvent . 

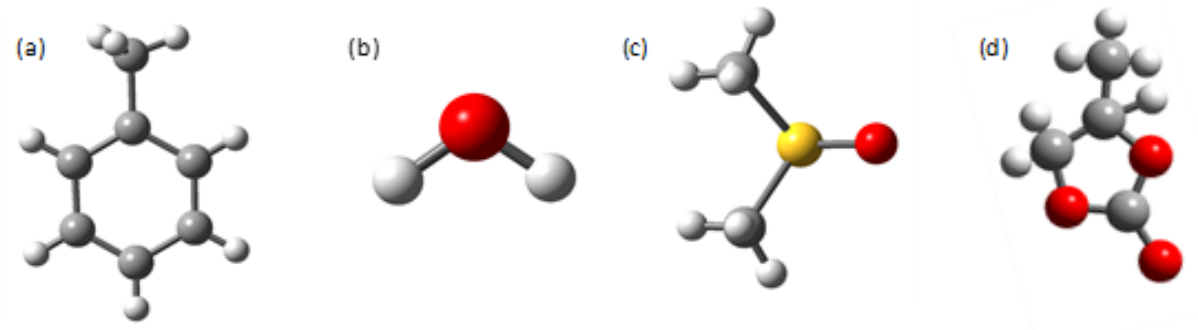

Figure 3.1. Molecular structure of (a) toluene. (b) water, (c) DMSO, and (d) PC.

Table 3.1. Dielectric constant and dipole moment of the used solvents.

\begin{tabular}{lll}
\hline Solvents & Dielectric Constant & Dipole Moment \\
\hline Toluene & 2.38 & 0.36 \\
Water & 80 & 1.85 \\
DMSO & 46.2 & 3.96 \\
PC & 64 & 4.9 \\
\hline
\end{tabular}

\subsection{Results and Discussion}

\subsubsection{SEM and XRD Results}

Nanostructures were synthesized using the liquid vapor process as described in Chapter 1. For this work, the FF stock solution was diluted with different solvents includng toluene, DI water, DMSO, and PC. All the measurements were accomplished from the drop casted film. Fresh solutions of nanostructures were used for drop casting. Figure $3.2(a-b),(c-d)$, (e-f), and (g-h) show the SEM images of synthesized peptide nanostructures using toluene, DI water, DMSO, and PC respectively. The sample synthesized using toluene forms small nanotubes with uniform diameter. The length of these tubes is about $15 \mu \mathrm{m}$. Nanotubes were formed with DI water where the diameter is not as uniform as nanotube synthesized using toluene. The range of the length of these nanotubes is about 50-500 $\mu \mathrm{m}$. DMSO solution results in rectangular plate like micro/nanostructures where the largest surface area varies from $1 \mu \mathrm{m} \times 1 \mu \mathrm{m}$ to $50 \mu \mathrm{m} \times 50 \mu \mathrm{m}$. PC solution gives longer nanotubes structure like DI water. 

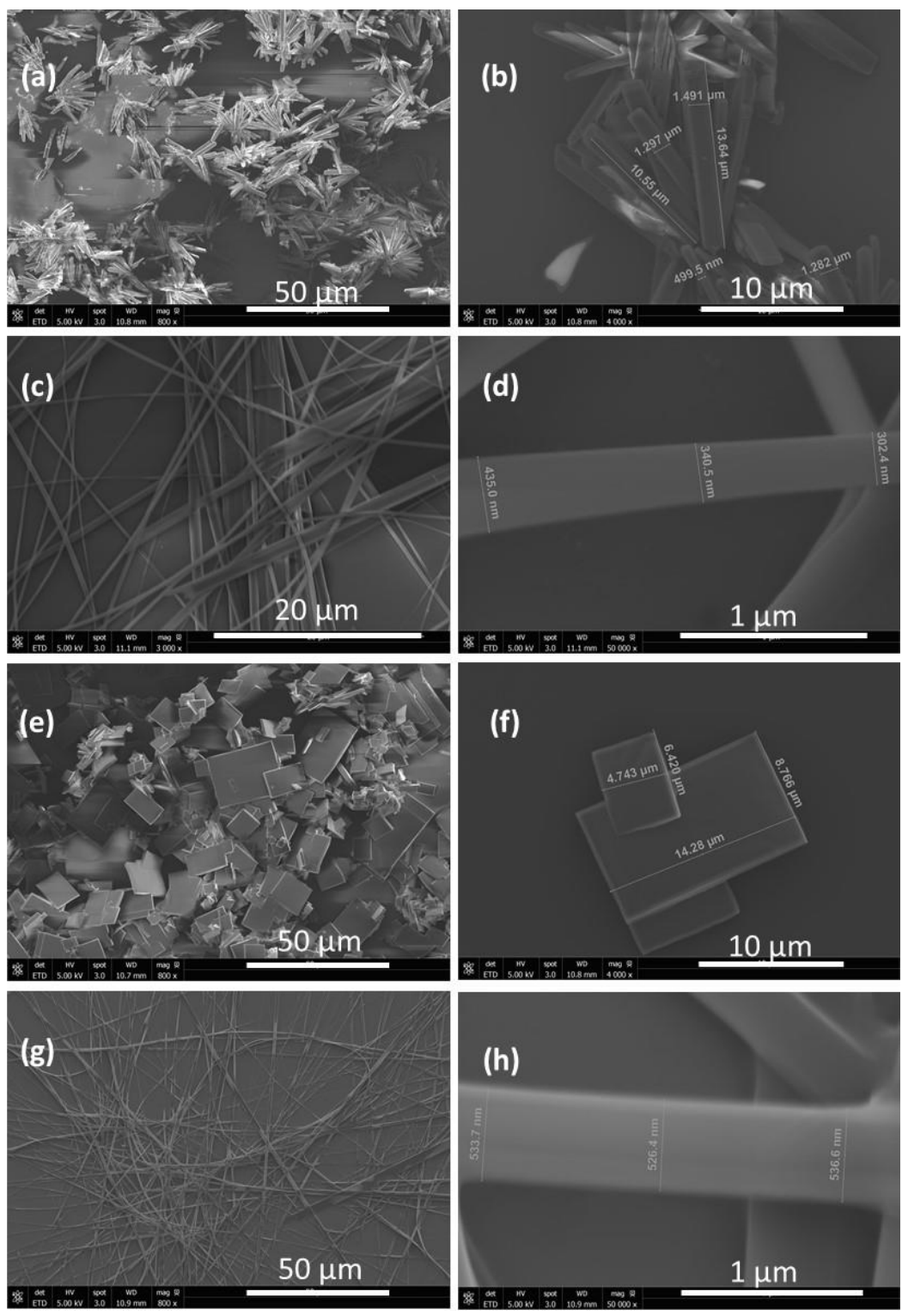

Figure 3.2. SEM images of FF-MNs synthesized using (a-b) toluene, (c-d) DI water, (e-f) DMSO, and (g-h) PC.

The XRD data was collected from the drop casted films on glass slide. Figure 3.3 shows the XRD patterns for the nanostructures obtained with varying solutions. Patterns are very different from each other. As seen from the XRD data, the peak positions and the relative intensity change with varying solvents. These data confirm that the crystal structure change with varying solvents. XRD data were analyzed using Rietveld refinement. Table 3.2 reports the lattice parameter with varying solvents. Refinement data confirm that 
nanostructures synthesized using toluene, DI water, and DMSO have hexagonal crystal structure whereas nanostructures synthesized using PC have orthorhombic phase. Toluene has larger lattice parameter compared to DI water and DMSO.

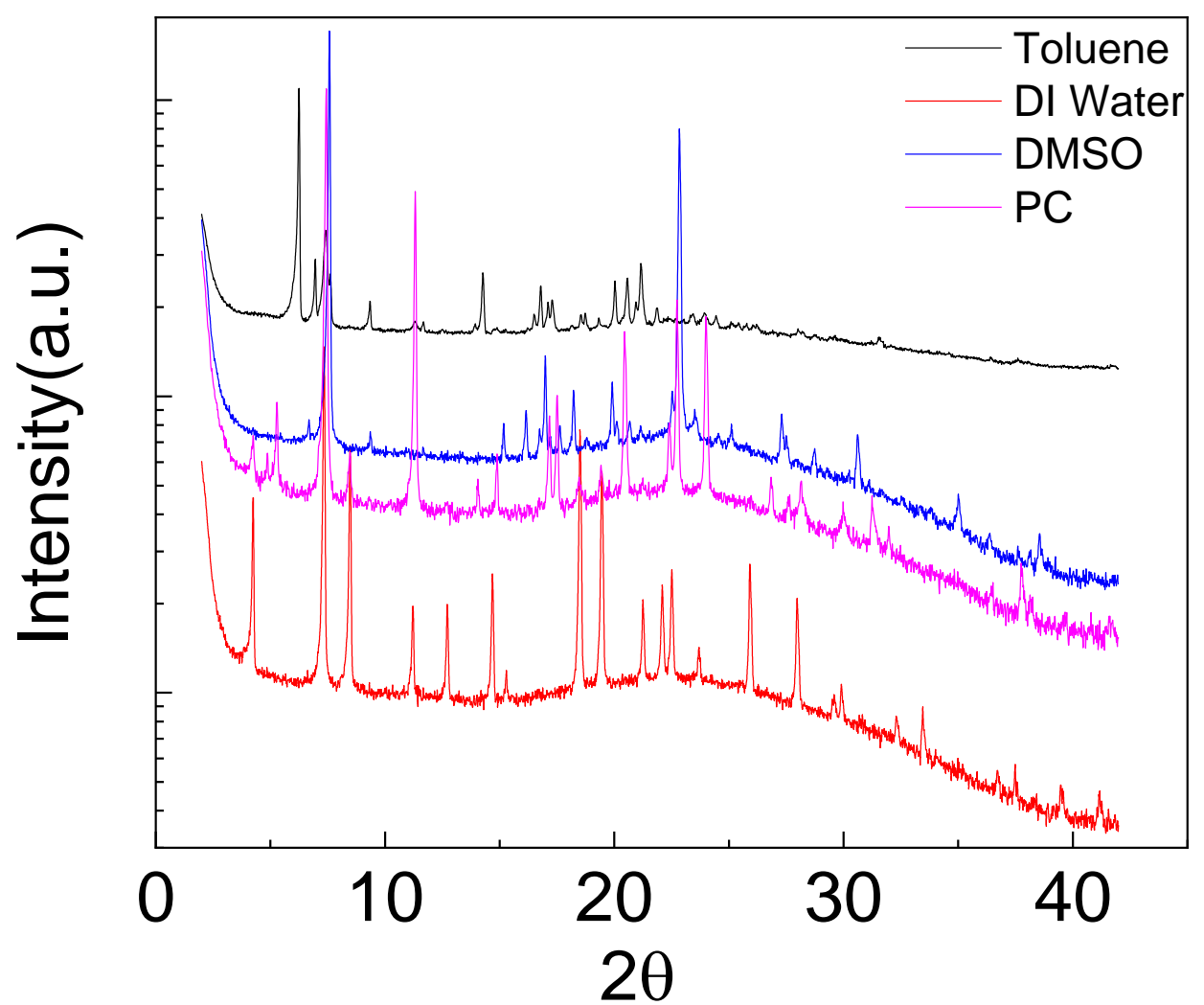

Figure 3.3. XRD pattern of FF-MNS nanostructures synthesized using different solvents.

Table 3.2. Lattice parameters from the nanostructures with varying solvents.

\begin{tabular}{lllll}
\hline Solvents & $\begin{array}{c}\text { Crystal } \\
\text { Structure }\end{array}$ & $\mathrm{a}\left(\mathrm{A}^{\circ}\right)$ & $\mathrm{b}\left(\mathrm{A}^{\circ}\right)$ & $\mathrm{c}\left(\mathrm{A}^{\circ}\right)$ \\
\hline Toluene & Hexagonal & 24.2054 & & 5.4749 \\
DI Water & Hexagonal & 24.1936 & & 5.4669 \\
DMSO & Hexagonal & 24.1296 & & 5.4688 \\
PC & Ortorhombic & 5.8713 & 10.5895 & 23.1244 \\
\hline
\end{tabular}

\subsubsection{Raman Spectroscopy with Varying Solvents}

Raman scattering data shows notable changes in the spectra with different solvents. Figure 3.4 shows the Raman spectra with varying solvent in four regions; (a) $65-400 \mathrm{~cm}^{-1}$, (b) 
$400-800 \mathrm{~cm}^{-1}$ (c) $800-1200 \mathrm{~cm}^{-1}$ and (d) $1200-1700 \mathrm{~cm}^{-1}$. Table 3.3 reports the Raman frequencies and the corresponding dependence pattern. As seen from the table, the frequencies change with varying solvent. The mode related to $\mathrm{NH}_{3}$ vibration shifts upward (Stokes spectrum) in frequencies for DMSO and toluene compared to DI water. On the other hand, frequencies corresponding to the $\mathrm{C}=\mathrm{O}$ vibration modes shifts to lower frequencies (Stokes spectrum) for DMSO and toluene. The Raman peak at $168 \mathrm{~cm}^{-1}$ in DI water corresponds to in plane rocking vibration of $\mathrm{NH}_{3}$ group in peptide nanostructures. This peak shifts to $175 \mathrm{~cm}^{-1}$ and $171 \mathrm{~cm}^{-1}$ in DMSO and toluene respectively. The Raman peak at $233 \mathrm{~cm}^{-1}$ in DI water corresponds to in plane bending vibration of $\mathrm{NH}_{3}$ group in peptide nanostructures. This peak shifts to $251 \mathrm{~cm}^{-1}$ and $249 \mathrm{~cm}^{-1}$ in DMSO and toluene respectively. The vibration mode at $1002 \mathrm{~cm}^{-1}$ arises from the breathing mode of the phenyl rings. The $660 \mathrm{~cm}^{-1}$ and $1505 \mathrm{~cm}^{-1}$ peaks signify orthorhombic phase of the nanostructures synthesized using PC solvent. The $1505 \mathrm{~cm}^{-1}$ peak is seen in the FTIR spectra; it is most likely that this peak becomes Raman active in the orthorhombic phase. The Raman peak at $1603 \mathrm{~cm}^{-1}$ in DI water corresponds to stretching vibration of $\mathrm{C}=\mathrm{O}$ in peptide nanostructures. This peak shifts to $1601 \mathrm{~cm}^{-1}$ and $1602 \mathrm{~cm}^{-1}$ in DMSO and toluene respectively. These changes in Raman frequencies can be correlated to change in the bond length as discussed in Section 3.3. 

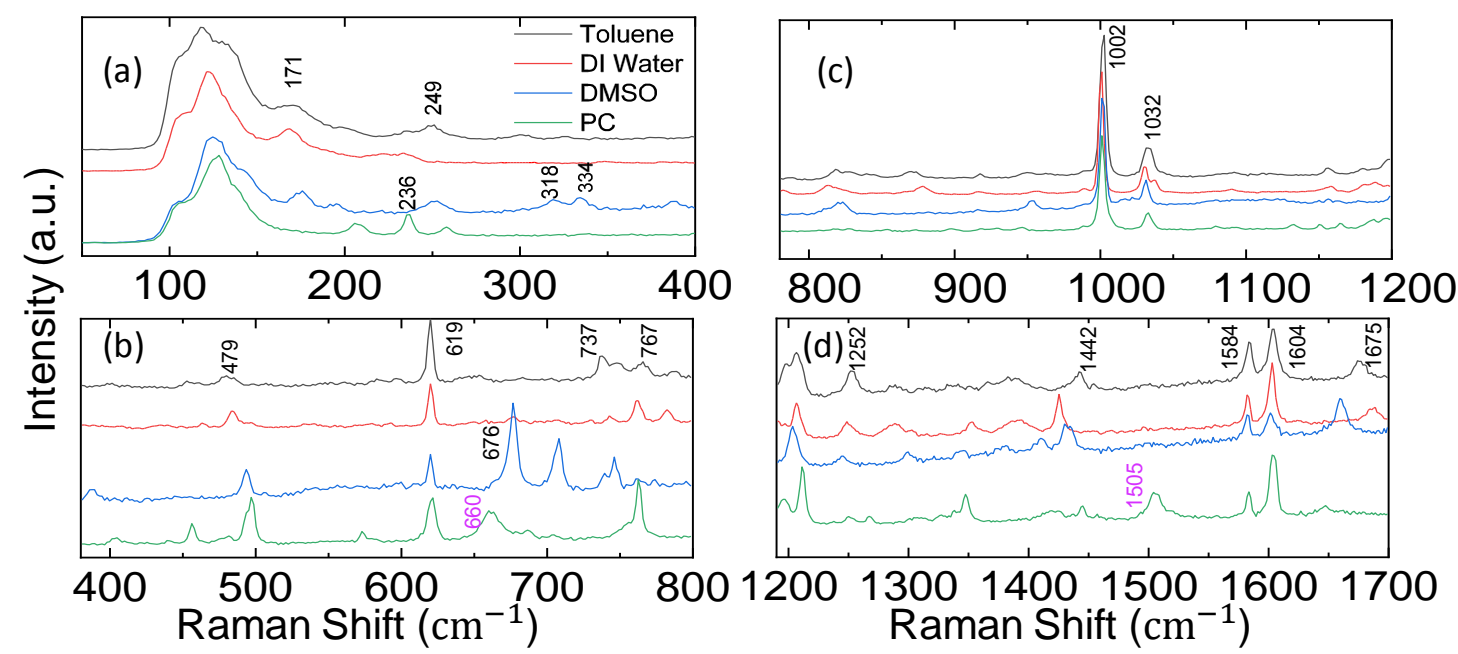

Figure 3.4. Raman spectra from FF-MNs nanostructures in (a) $65-400 \mathrm{~cm}^{-1}$, (b) $400-800$ $\mathrm{cm}^{-1}$ (c) $800-1200 \mathrm{~cm}^{-1}$ and (d) $1200-1700 \mathrm{~cm}^{-1}$.

Table 3.3. Raman peak position and identification of the vibration mode.

\begin{tabular}{|c|c|c|c|}
\hline $\begin{array}{l}\text { Peak Position in } \\
\text { DI Water } \mathrm{cm}^{-1}\end{array}$ & $\begin{array}{l}\text { Peak Position in } \\
\text { DMSO } \mathrm{cm}^{-1}\end{array}$ & $\begin{array}{l}\text { Peak Position } \\
\text { in Toluene } \mathrm{cm}^{-1}\end{array}$ & Vibration Mode \\
\hline 168 & 175 & 171 & $\begin{array}{l}\text { In plane Rocking } \\
\text { Vibration of } \mathrm{NH}_{3} \\
\text { Group }\end{array}$ \\
\hline 233 & 251 & 249 & $\begin{array}{c}\text { Bending } \\
\text { Vibration } \mathrm{NH}_{3} \\
\text { Group }\end{array}$ \\
\hline No Peak & 318 & 299 & $\begin{array}{c}\text { Bending } \\
\text { Vibration of } \mathrm{NH}_{3} \\
\text { group, } \mathrm{C}=\mathrm{O} \text { group, } \\
\text { and peptide bond } \\
\text { Bending }\end{array}$ \\
\hline No Peak & 333 & 324 & $\begin{array}{l}\text { Vibration of } \mathrm{NH}_{3} \\
\text { group, } \mathrm{C}=\mathrm{O} \text { group, } \\
\text { and peptide bond }\end{array}$ \\
\hline No Peak & 387 & No peak & $\begin{array}{c}\text { Bending } \\
\text { Vibration of } \mathrm{NH}_{3}\end{array}$ \\
\hline 464 & No Peak & 453 & Not Sure \\
\hline 484 & 492 & 479 & $\begin{array}{c}\text { Torsional } \\
\text { Vibration of } \mathrm{NH}_{3}\end{array}$ \\
\hline No Peak & 676 & No Peak & $\begin{array}{l}\text { Out of plane } \mathrm{NH} \\
\text { bending }\end{array}$ \\
\hline No Peak & 707 & No Peak & $\begin{array}{l}\text { Out of plane } \mathrm{NH} \\
\text { bending }\end{array}$ \\
\hline
\end{tabular}




\begin{tabular}{|c|c|c|c|}
\hline 878 & No Peak & 870 & $\begin{array}{l}\text { Out of plane } \mathrm{NH} \\
\text { bending }\end{array}$ \\
\hline 956 & 952 & 948 & $\begin{array}{c}\text { Bending } \\
\text { Vibration of } \mathrm{NH}_{3} \\
\text { group, } \mathrm{C}=\mathrm{O} \text { group, } \\
\text { and peptide bond } \\
\text { Symmetric }\end{array}$ \\
\hline 1206 & 1201 & 1205 & $\begin{array}{l}\text { Stretching of the } \\
\text { Phenol Ring }\end{array}$ \\
\hline 1250 & 1243 & 1251 & $\begin{array}{l}\text { CN Stretching } \\
\text { and NH Bending }\end{array}$ \\
\hline 1289 & 1297 & 1286 & $\begin{array}{l}\text { CN Stretching } \\
\text { and NH Bending }\end{array}$ \\
\hline 1603 & 1601 & 1602 & $\begin{array}{c}\text { Stretching } \\
\text { Vibration of } \mathrm{C}=\mathrm{O}\end{array}$ \\
\hline 1686 & 1659 & 1674 & $\begin{array}{c}\text { Stretching } \\
\text { Vibration of } \mathrm{C}=\mathrm{O}\end{array}$ \\
\hline
\end{tabular}

\subsection{Theoretical Calculation}

To understand the structural changes observed in SEM and Raman scattering data with varying solvents, optimization and vibration calculations in presence of varying solvents were executed using DFT method. Optimized structures of the FF monomer unit with different solvents were calculated using DFT method and 6-31+G (d, p) basis set. Gaussian 09 software was used for the DFT calculation, see chapter 2. ${ }^{22}$ Toluene, DI water, DMSO, and PC solvents were used for the solvation of FF molecule. Solvation Model based on Density (SMD) was used for the solvation. SMD is an implicit solvation model where the main parameter is the dielectric constant. The value of the dielectric constant defines the degree of polarizability of the solvent. For implicit solvents, a solute is encapsulated in a cavity and then the cavity is placed in homogeneously polarizable continuum media describing the solvent. 


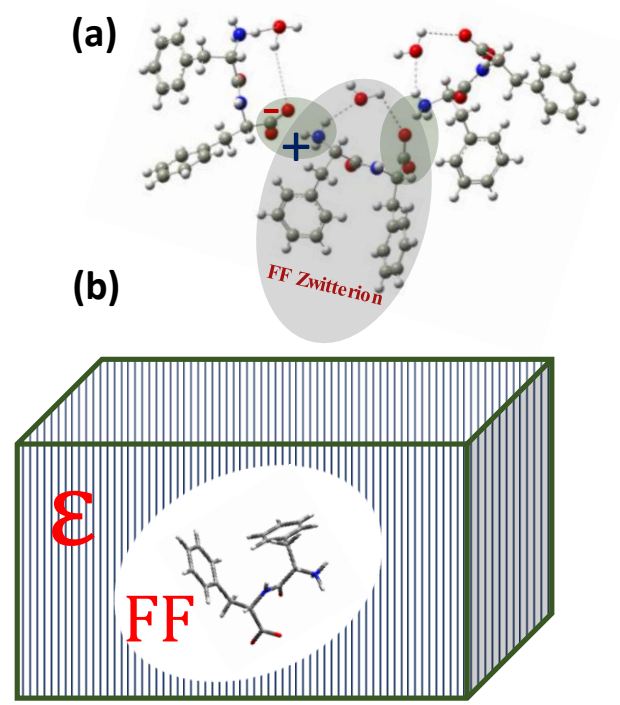

(c)

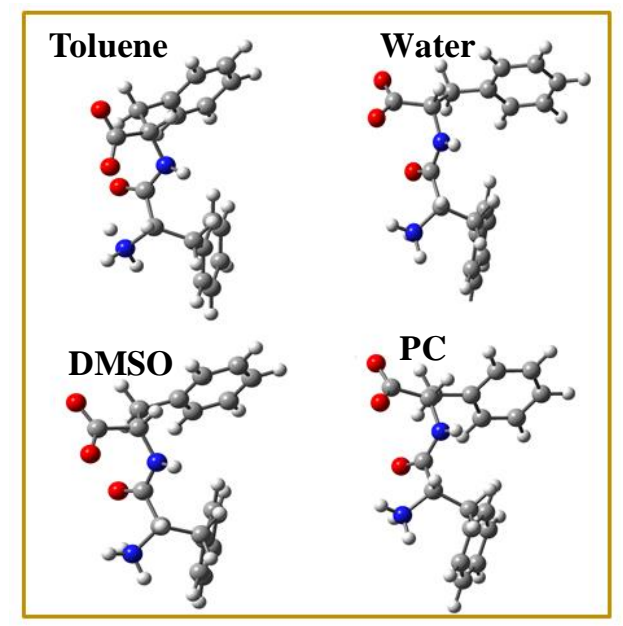

Figure 3.5. (a) Shows the arrangement of three basic unit of peptide nanostructures in the crystal of the peptide nanostructures Solvation model used for DFT calculation.

The solute's charge distribution meets the continuous dielectric field at the surface of the cavity and polarizes the surrounding medium, which causes a change in the polarization on the solute. Figure 3.5 (b) shows the solvation model. Vibration spectra from all the optimized structures were calculated. The Raman spectra was calculated from the Raman activity, for more details, we refer chapter $2 .{ }^{24}$ Figure 3.5 (c) shows the optimized structures of FF molecule in different solvents.

FF is a zwitterionic molecule where positive charge is on the $\mathrm{NH}_{3}$ group and negative charge is on the $\mathrm{COOH}$ group. Figure 3.5 (a) shows the arrangement of three basic unit of peptide nanostructure in the crystal of the peptide nanostructure. FF based peptide has abundance number of sites including amide groups in the peptide backbone and amino and carboxyl groups for hydrogen bond formation. Charges on the $\mathrm{NH}_{3}$ group and $\mathrm{COOH}$ group contribute to the electrostatic interaction. Aromatic rings in the FF molecule take part in $\pi-\pi$ stacking or hydrophobic interaction. 
Optimization of FF monomer unit in presence of different solvents using DFT provide insight into the hydrogen bonding capability of $\mathrm{NH}_{3}$ group and $\mathrm{COOH}$ group and their changes after interaction with the solvents. There is considerable change in the charge distribution of the FF molecule after the interaction with the solvents, which signify changes in hydrogen bonding capability, electrostatic interaction, and $\pi-\pi$ interaction between aromatic rings. FF has the highest dipole moment in water and the lowest dipole moment in Toluene. Table 3.4 lists the charges on the amine group, the carboxyl group and the aromatic ring. The charge on amine and carboxyl group has important effect, as they are the linker between two FF units. Change in the charge distribution in the aromatic ring affects the $\pi-\pi$ interaction. Table 3.5 reports the optimization energy, dipole moment and entropy of the FF molecule in the presence of varying solvents. The optimized energies yield insight of the stability of the structures; molecule with low optimized energy is the most stable structure in the solvents.

In case of toluene driven self-assembly, intermolecular electrostatic interaction, and hydrogen bonding ability are less compared to other solvents. The dipole moment and charges are small in the FF molecule in the presence of toluene. These affect the intermolecular hydrogen bonds formation. From the molecular structure of toluene, it is clear that the chance of solvent-bridged hydrogen bond formation is low. The lowest optimization energy confirms that the FF is not stable in toluene solution. However, $\pi-\pi$ stacking, which dominates in toluene, results in small nanostructures formation as shown in Figure $3.2(a-b)$.

Intermolecular electrostatic interaction, hydrogen bonding ability, and solvent bridged hydrogen bond are very strong compared to other solvents in water driven self-assembly. 
Dipole moment and charges are larger in FF in water compared to other solvents. The solvent bridged hydrogen bond formation in water is strong since water molecule has the affinity to form hydrogen bond with an amphiphilic molecule. As a result, the optimization energy of the FF molecule in water is much less compared to toluene and DMSO. Strong electrostatic interaction, hydrogen bonding ability, and solvent bridged hydrogen bond results in long order nanotube structure as show in Figure 3.2 (c-d).

Intermolecular electrostatic interaction, and intermolecular hydrogen bonding ability have strong contribution in DMSO driven self-assembly. However, the solvent bridged hydrogen bond is not strong. From the molecular structure of DMSO, it is clear that this solvent has less capability to form hydrogen bond with FF molecule, which increases optimization energy compared to water and PC. As the solvent bridged hydrogen bond is not strong in DMSO driven self-assembly, it forms plate like structure rather than nanotubes as shown in Figure 3.2 (e-f).

In case of PC driven self-assembly, intermolecular electrostatic interaction, hydrogen bonding ability, and solvent bridged hydrogen bond are responsible for nanostructures formation. From the molecular structure, it is clear that the solvent has strong affinity to form hydrogen bond with FF molecule, which results in minimum optimization energy. These strong interactive forces in PC solvent provide the most stable crystal structure which is the orthorhombic phase, shown in Figure $3.2(\mathrm{~g}-\mathrm{h}) .{ }^{29}$ As mentioned in Section 3.2.2, the Raman scattering data also confirms the orthorhombic phase of the nanostructures using PC solvent. 
Table 3.4. Charge distribution of FF monomer unit in different solvents.

\begin{tabular}{ccccc}
\hline Molecule & $\begin{array}{c}\text { Charge }\left(\mathrm{NH}_{3}\right. \\
\text { group })\end{array}$ & $\begin{array}{c}\text { Charge }(\mathrm{COO}- \\
\text { group })\end{array}$ & \multicolumn{2}{c}{$\begin{array}{c}\text { Charge (two } \\
\text { aromatic rings })\end{array}$} \\
\hline FF in Toluene & 0.589 & -0.619 & 0.329, & 0.950 \\
FF in Water & 0.726 & -0.902 & 0.375, & 0.362 \\
FF in DMSO & 0.707 & -0.748 & 0.295, & 0.203 \\
FF in PC & 0.707 & -0.75 & 0.297, & 0.196 \\
\hline
\end{tabular}

Table 3.5. List of minimum energy and entropy of FF monomer unit under varying solvents.

\begin{tabular}{ccc}
\hline Molecules & $\begin{array}{c}\text { OptimizedEnergy } \\
\text { (Hartree) }\end{array}$ & $\begin{array}{c}\text { Dipole Moment of FF } \\
\text { molecules in } \\
\text { solvents(Debye) }\end{array}$ \\
\hline FF in Toluene & -1033.2356 & 18.8382 \\
FF in water & -1033.2837 & 25.4648 \\
FF in DMSO & -1033.2698 & 23.7081 \\
FF in PC & -1033.2867 & 23.6379 \\
\hline
\end{tabular}

Vibrational frequency calculation was done from the optimized structures of FF in various solvents. The Raman activity and bond length were obtained from the DFT calculations. Experimentally we observe change in Raman shift corresponding to the bending vibration of $\mathrm{NH}_{3}$ group at $235 \mathrm{~cm}^{-1}$ with varying solvents. Figure 3.6 (a) shows the change in the bending vibration of the $\mathrm{NH}_{3}$ group with varying solvents, which support experimental data in Section 3.2.3. In addition, the vibration frequency at $1645 \mathrm{~cm}^{-1}$ (experimentally $1603 \mathrm{~cm}^{-1}$ ) corresponding to the stretching vibration of $\mathrm{C}=\mathrm{O}$, change with solvents. Figure 3.6 (b) shows the change in frequency of the stretching vibration of $\mathrm{C}=\mathrm{O}$ under various solvents which coincide with the experimental Raman data. The Table in Figure 3.6 (c) reports the bond lengths of FF molecule optimized under different solvents. Bond lengths of -N-H- (of $\mathrm{NH}_{3}$ group) are smaller in DMSO, and toluene compared to DI and PC water. On the other hand, bond lengths of (NH)-C-C-(OO) are larger in DMSO, 
toluene, and PC compared to DI water. Decreasing bond length of $-\mathrm{N}-\mathrm{H}-$ harden the vibration mode of DI water at $235 \mathrm{~cm}^{-1}$ (experimentally $233 \mathrm{~cm}^{-1}$ ) to $245 \mathrm{~cm}^{-1}$ and $248 \mathrm{~cm}^{-}$ ${ }^{1}$ in DMSO and PC respectively. Softening of stretching vibrations of $\mathrm{C}=\mathrm{O}$ in DMSO and PC compared to DI water are raised because of increasing bond length as seen in the table (Figure $3.6(\mathrm{c})$ ).

(a)

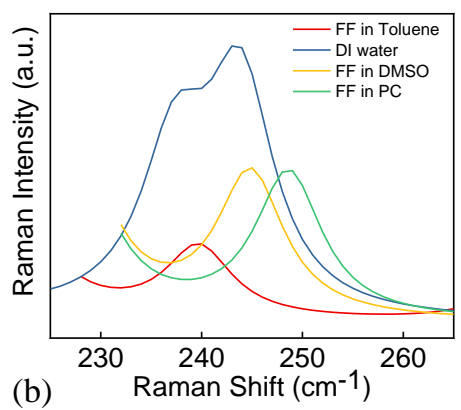

(c)

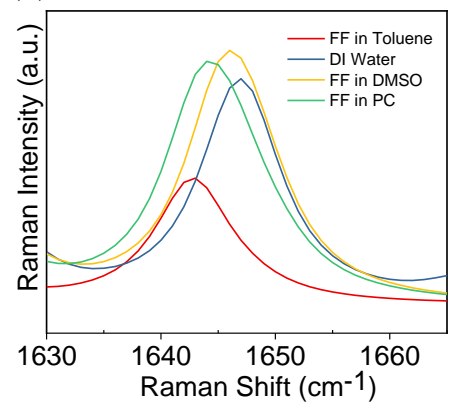

\begin{tabular}{lllll}
\hline Bond & DI Water & DMSO & Toluene & PC \\
\hline (H-)-N-C-(-COO) & 1.468 & 1.470 & 1.481 & 1.471 \\
(H-)-N-C-(=O) & 1.339 & 1.343 & 1.341 & 1.344 \\
N-H (of NH N $_{3}$ & $\mathbf{1 . 0 2 6}$ & $\mathbf{1 . 0 2 5}$ & $\mathbf{1 . 0 2 1}$ & $\mathbf{1 . 0 2 6}$ \\
(NH)-C-C-(OO) & $\mathbf{1 . 5 6 3}$ & $\mathbf{1 . 5 8 0}$ & $\mathbf{1 . 5 8 8}$ & $\mathbf{1 . 5 8 1}$ \\
\hline
\end{tabular}

Figure 3.6. . (a) and (b) Calculated Raman spectra. (c) Table reporting the bond lengths of FF molecule optimized in varying solvents.

\subsection{Conclusions}

Solvent driven nanostructures formation shed light on the contribution of the noncovalent interactions including electrostatic, hydrogen bonding, and $\pi-\pi$ interaction, in the self-assembly process of FF-MNS nanostructures. The Basic building block FF, is changed in different solvents altering the interaction forces responsible for nanostructures formation. SEM data shows clear changes in the shape and size with varying solvents. XRD data along with Rietveld analysis confirm the changes in the crystal structures. The Raman 
scattering data add to the insight of the structural changes with varying solvents. It shows that bond polarization of $\mathrm{NH}_{3}$ bond and Carboxyl -COO- groups of FF-MNS changes in presence of different solvents. DFT calculation of the basic building block of FF-MNS nanostructures provides significant information about how the different interaction forces contribute to form nanostructures with various shape and size. Theoretical calculation also supports that bond polarization and bond length changes for $\mathrm{NH}_{3}$ and -COO- group in different solvents. This work adds important understanding of the solvent driving mechanism of self-assembly process to the prior work based on contribution of various forces on the self-assembly process. 


\section{Probing Nonlinear Properties of FF-MNS Using SHG}

We have explored the non-linear properties of FF-MNS nanostructures in this chapter. The work by Handelman et al. showed that the effective nonlinearity $\left(2^{\text {nd }}\right.$ order nonlinearity of quartz times the power efficiency of FF nanostructures compared to the power efficiency of the quartz in SHG measurement) of FF nanostructures is almost 30 times higher than that of quartz. ${ }^{19}$ However, the detailed analysis of the non-linear properties has not been investigated. The focus of this chapter is to study the diameter dependent and temperature dependent non-linear properties and correlate the non-linear properties with the structure.

\subsection{Introduction}

The as-synthesized FF-MNS possess a $P 6_{1}$ non-centrosymmetric space group. ${ }^{30}$ This odd-tensor space group is the basis for the existence of several physical properties such as ferroelectricity, ${ }^{31-32}$ piezoelectricity, ${ }^{18}$ and optical activity, and thus serves as a potential platform for nonlinear optics. ${ }^{19,30}$ The polar hexagonal form of FF-MNS is known to show an irreversible phase transition at $\sim 150{ }^{\circ} \mathrm{C}$ to a non-polar orthorhombic structure. ${ }^{33}$ Recently, there have been several reports of using SHG polarimetry for structural characterization of II-VI and III-V semiconducting nanostructures, ${ }^{34-36}$ and even monolayers of transition metal dichalcogenides. ${ }^{37}$

Although several works have reported on the nonlinear optical properties of FF nanostructures (tubes, vesicles, and belts), thus far there is no experimental determination of the individual nonlinear optical coefficients of FF nanostructures. Herein, we report on the second-order nonlinear optical coefficients of FF-MNS synthesized by a liquid phase method. The tubes were diluted such that individual FF-MNS could be probed by SHG, scanning electron microscopy (SEM), and confocal Raman microscopy. The SHG 
properties of individual FF-MNTs, here we denote the individual tube by FF-MNTs rather than FF-MNS, are characterized as a function of the diameter of the tubes. With an incoming linearly polarized fundamental frequency of light incident on the sample in transmission geometry, the SHG signal was collected both for $p$ - and $s$ - polarization (the nomenclature for these two polarizations are described in the experimental setup, chapter 2). The s-polarization dependence of the SHG signal is similar for all diameters, however, the p-polarization dependent SHG pattern changes as a function of the diameter of the tubes. In the $P 6_{1}$ symmetry, there are two transverse components $\left(\mathrm{d}_{15}\right.$ and $\left.\mathrm{d}_{31}\right)$ perpendicular to tube axis and the coefficient along the tube axis is represented as $\mathrm{d}_{33}$. The ratio of the transverse components to the longitudinal component of the nonlinear coefficient: $d_{15} / d_{33}$ and the magnitude of $d_{31} / d_{33}$ increases with the diameter (or the edge length) of the hexagonal tubes. Subtle changes in the Raman spectrum are observed in the $80 \mathrm{~cm}^{-1}$ region as a function of the diameter of the tubes, highlighting interactions between FF molecules and water.

SHG polarimetry from thermally treated FF-MNTs show a similar behavior as increasing the diameter of the tubes; both the transverse components of the nonlinear coefficients increase by thermally annealing the tubes till $\sim 140{ }^{\circ} \mathrm{C}$, beyond which the overall SHG intensity decreases. Once the FF-MNTs undergo a phase change to the orthorhombic phase at $150{ }^{\circ} \mathrm{C}$, the SHG signal completely disappears.

\subsection{Symmetry and d Tensor in FF-MNS}

To clearly explain our results of SHG polarimetry, let's begin with the definitions of coordinates and the relationship between the experimental data and SHG coefficient of the FF-MNTs in our experimental conditions. In general, the second-order nonlinear $d$ 
coefficients, which are related to the second-order susceptibility, are expressed as a tensor representation, for details we refer to Chapter 2. Since the FF-MNTs belong to a symmetry system with $P 6_{1}$ space group, using the Kleinman symmetry, ${ }^{38}$ the relationship between the induced SHG polarization and the applied fundamental field is given by

$$
\left(\begin{array}{l}
P_{x^{\prime}}(2 \omega) \\
P_{y^{\prime}}(2 \omega) \\
P_{z^{\prime}}(2 \omega)
\end{array}\right)=\left(\begin{array}{cccccc}
0 & 0 & 0 & d_{14} & d_{15} & 0 \\
0 & 0 & 0 & d_{15} & -d_{14} & 0 \\
d_{31} & d_{31} & d_{33} & 0 & 0 & 0
\end{array}\right)\left(\begin{array}{c}
E_{x^{\prime}}^{2}(\omega) \\
E_{y^{\prime}}^{2}(\omega) \\
E_{z^{\prime}}^{2}(\omega) \\
2 E_{y^{\prime}}(\omega) E_{z^{\prime}}(\omega) \\
2 E_{x^{\prime}}(\omega) E_{z^{\prime}}(\omega) \\
2 E_{x^{\prime}}(\omega) E_{y^{\prime}}(\omega)
\end{array}\right)
$$

where $\left(x^{\prime}, y^{\prime}, z^{\prime}\right)$ are the Cartesian coordinates associated with the sample frame, and $E_{x},(\omega)$, $E_{y}(\omega)$, and $E_{z}(\omega)$ are the incident electric field components oscillating at the frequency $\omega$.

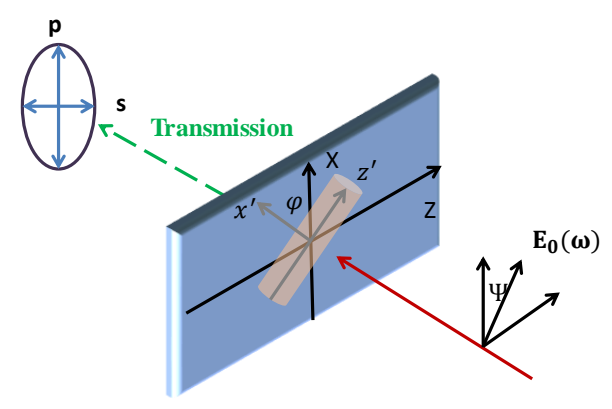

Figure 4.1. Schematic of the lab frame and the sample frame.

The incident electric field components in the $(\mathrm{X}, \mathrm{Y}, \mathrm{Z})$ laboratory frame are then expressed as

$$
\boldsymbol{E}=E_{0} \cos \Psi \widehat{X}+E_{0} \sin \theta \sin \Psi \widehat{Y}+E_{0} \cos \theta \sin \Psi \widehat{Z},
$$

where $\theta$ is the angle between the $\mathrm{Y}$-axis and laser propagation direction, and $\Psi$ is the angle of the incident polarization, shown in Figure 4.1. We performed SHG polarimetry for both $\theta=0^{\circ}$ and $\theta=45^{\circ}$. The results are similar except a relative SHG value difference due to the Fresnel coefficients of the fundamental and SHG waves. To simplify the process, we select $\theta=0^{\circ}$, which results in the $\hat{Y}$ component to be 0 . In this geometry, the polarization dependent linear Fresnel coefficients can also be simplified. From the SEM images we 
confirmed that the $x^{\prime}$ and $z^{\prime}$ axes of the tubes are in the sample plane in our experiment. The electric field components in the lab frame are given by:

$$
\begin{gathered}
E_{X}=E_{0} \cos \Psi, \\
E_{Z}=E_{0} \cos \theta \sin \Psi \widehat{Z}, \\
E_{Y}=0
\end{gathered}
$$

Using $\varphi$ as the angle between the laboratory and FF-MNT reference frames and equation (4.3) (4.4) and (4.5), the electric field components in the sample's reference frame are:

$$
\begin{gathered}
E_{x^{\prime}}=E_{X} \cos \varphi-E_{Z} \sin \varphi=E_{0} \cos (\Psi+\varphi), \\
E_{Z^{\prime}}=E_{X} \sin \varphi+E_{Z} \cos \varphi=E_{0} \sin (\Psi+\varphi), \\
\text { and } E_{y^{\prime}}=0 .
\end{gathered}
$$

From equation (4.1), the polarization components are thus:

$$
\begin{gathered}
P_{x^{\prime}}=2 d_{15} E_{x^{\prime}} E_{z^{\prime}}=d_{15} E_{0}^{2} \sin 2(\Psi+\varphi), \\
P_{z^{\prime}}=d_{31} E_{x^{\prime}}^{2}+d_{31} E_{z^{\prime}}^{2}=d_{31} E_{0}^{2} \cos ^{2}(\Psi+\varphi)+d_{33} E_{0}^{2} \sin ^{2}(\Psi+\varphi) .
\end{gathered}
$$

In our setup, the FF-MNTs were oriented such that the nanotube $z^{\prime}$ axis was parallel to the X-axis $\left(\varphi=90^{\circ}\right)$. We define the $s$-polarization as the SHG polarization being perpendicular to the plane of incidence at $\theta=45^{\circ}$. Therefore, at normal incidence $\theta=0^{\circ}$, the $s$-polarization is perpendicular to the nanotube axis. The SHG intensity in this configuration is given by

$$
I_{S}=\left|P_{x}\right|^{2}=\left(d_{15} E_{0}^{2} \sin 2 \Psi\right)^{2} .
$$

Similarly, the P-polarization is defined when the SHG polarization is parallel to the nanotube $z^{\prime}$ axis. At normal incident, the SHG intensity simplifies to

$$
I_{p}=\left|P_{z^{\prime}}\right|^{2}=\left(d_{31} E_{0}^{2} \sin ^{2} \Psi+d_{33} E_{0}^{2} \cos ^{2} \Psi\right)^{2} .
$$




\subsection{Sample Preparation}

Synthesis process has been described in Chapter 1. The synthesized FF-MNTs tubes were diluted in isopropanol to separate the each individual tube. SHG and Raman measurement were performed from each separated tube.

\subsection{SHG Polarimetry as a Function of Tube Diameter}

The electric field at an angle $\Psi$ relative to the laboratory axis (Figure 4.1) was rotated by a $\lambda / 2$ wave plate from 0 to $180^{\circ}$ in front of the sample for measuring SHG polarimetry in both parallel and perpendicular polarizations with respect to the tube axis. Additionally, the SHG signal was also noted by rotating the sample along the vertical X-axis. To measure the SHG from an individual FF-MNS, a long working distance microscope was used to identify the tube and the YAG laser illumination on the tube along with visible light and a He-Ne laser. The YAG laser and He-Ne laser were co-linear in their optical path. A CCD camera was mounted on the microscope to monitor the sample for selecting individual FFMNS and determining the tube orientation during the measurement.

We captured $s$ - and $p$ - polarization SHG data by placing another rotating polarizer in front of the detector.The SHG intensity was analyzed in terms of both $s$ - and $p$-polarization from FF-MNTs of varying diameters at $\varphi=90^{\circ}$. Figure 4.2 (a-e) shows the SEM images of a few individual FF-MNTs that were used for this study. The approximate outer diameters (O.D.) of FF-MNTs were inferred from these images. Further, we also obtained the edge length of the hexagonal FF-MNTs, similar to what has been shown for organic single crystalline hexagonal micro-prisms. ${ }^{39}$ Figure 4.2 (f) captures a typical FF-MNT showing the entire length, which can be as long as a few hundred micrometers. Each FFMNT was aligned such that the tube axis was kept perpendicular to the $\mathrm{Z}$ axis; the 
orientation of the linear polarizer in front of the detector was changed to capture both $s$ and $p$ - polarization of the SHG response. This geometry ensures that we can use eqs 4 and 5 to fit the experimental data. Figure $4.2(\mathrm{~h}-\mathrm{j})$ shows the SHG signal for both $s$ - and $p$ polarizations for three different tube diameters. The $s$-polarization pattern is similar for all tubes with peaks observed at $45^{\circ}, 135^{\circ}, 225^{\circ}$, and $315^{\circ}$. The $p$-polarization pattern for small diameter tubes clearly show peaks at $0^{\circ}, 90^{\circ}, 180^{\circ}$, and $270^{\circ}$; with increasing diameter, the peaks at $0^{\circ}$ and $180^{\circ}$ decrease in intensity. Figure $4.2(\mathrm{~g})$ compares the $p$-polarization polarimetric plot of the normalized SHG intensity from two tubes; the smaller O.D. (8.5 $\mu \mathrm{m})$ sample shows a significant $\mathrm{SHG}$ intensity at $0^{\circ}$ and $180^{\circ}$ compared to the higher O.D. $(13.2 \mu \mathrm{m})$ sample.

The ratio of the overall intensity of $p$-polarization SHG to $s$-polarization SHG decreases with increasing diameter of the tubes, suggesting that one of the nonlinear coefficients in equation (5) is most likely negative. In order to gain a complete insight into the nonlinear coefficients as a function of the diameter (or the edge length) of the tubes, the individual SHG polarimetric data was fit with either equation (4.11) or (4.12). This results in obtaining the trends for the ratios of the nonlinear coefficients as a function of the tube diameter. We note that determining the absolute values of the nonlinear coefficients in our experiment is difficult since the optical constants of FF-MNTs are not known, which further complicates the comparison of the SHG intensity with a standard sample. We thus determine the ratio of the transverse components $\left(d_{31}\right.$ and $\left.d_{15}\right)$ to the longitudinal component, $d_{33}$. 

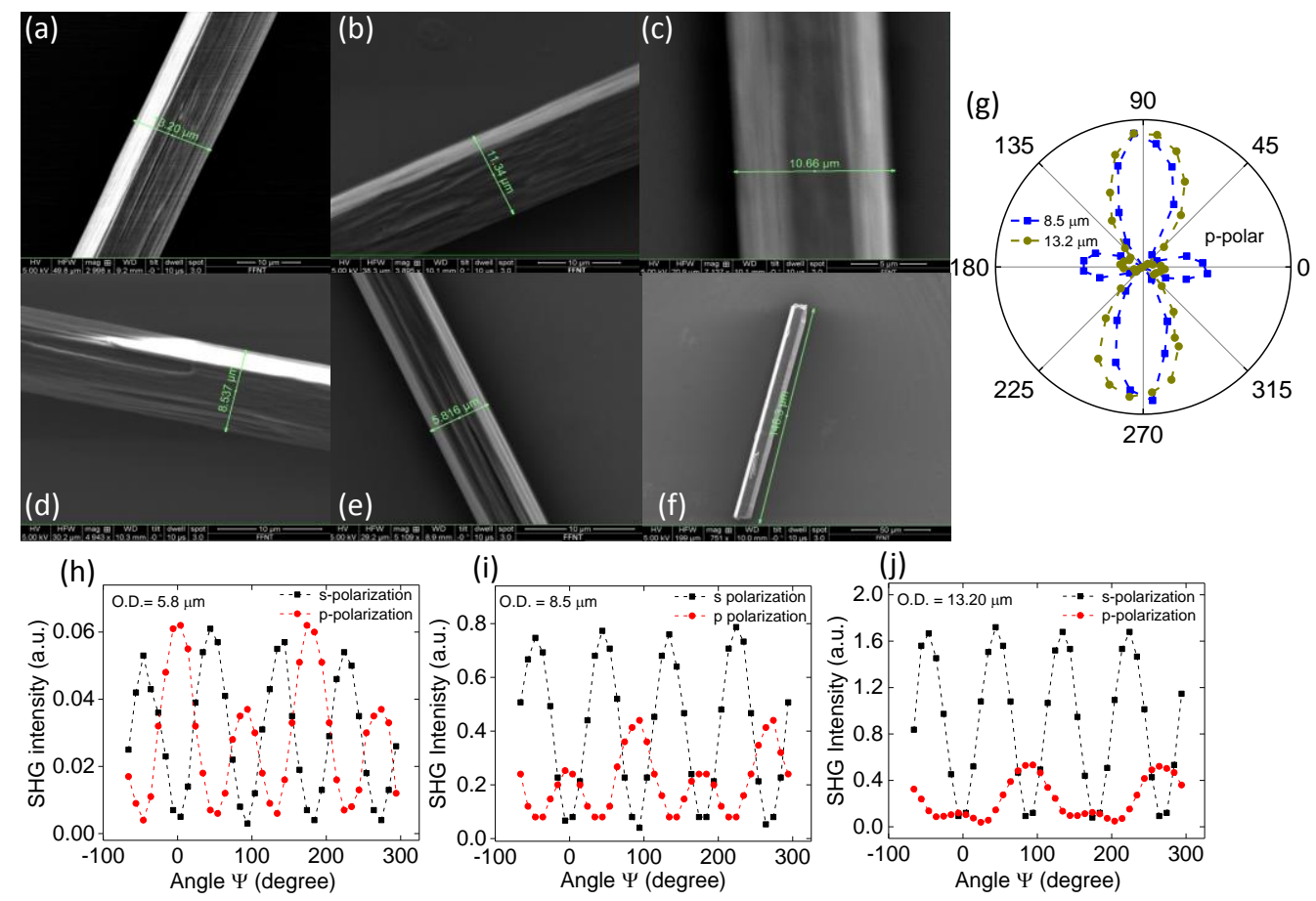

Figure 4.2. (a-e) SEM images of individual FF-MNTs showing the outside diameters. (f) SEM image of a FF-MNT with an overall length of $148 \mu \mathrm{m}$. (g) Experimental SHG polarimetric response from two FF-MNTs with different outer diameters for p-polarization. (h-j) Experimental SHG response from three different outer tube diameters: $5.8 \mu \mathrm{m}, 8.5 \mu \mathrm{m}$, and 13.2 $\mu \mathrm{m}$. In each case the sample was oriented such that the laboratory frame and the sample's reference frame coincided. The black symbol and dotted line correspond to s-polarization and the red symbol and dotted line correspond to p-polarization.

The $p$-polarization data was first fit with equation (4.12) to obtain the ratio of $\mathrm{d}_{31} / \mathrm{d}_{33}$. All fits yield a negative sign for this ratio. Using the individual values of $d_{31} / d_{33}$ for each tube obtained by fitting the $p$-polarization data, the total SHG intensity for each tube was fit by combining equation (4.11) and (4.12), yielding the value for $\mathrm{d}_{15} / \mathrm{d}_{33}$. This ratio was obtained to be positive, which implies that both coefficients $d_{15}$ and $d_{33}$ are positive or they are negative. Looking at some inorganic perovskites where both SHG and piezoelectric coefficients are known, the symmetry of the system governs both the longitudinal piezoelectric and SHG coefficients, which typically have positive values. We therefore assume $\mathrm{d}_{33}$ to be positive. For $p$-polarization at non-zero incident angles, all three 
coefficients survive; with negative values for both $d_{15}$ and $d_{33}$ one cannot reconcile with the specific SHG pattern. This suggests that both $d_{33}$ and $d_{15}$ have positive values. Thus, the nonlinear coefficient, $d_{31}$, is negative and $d_{33}$ is positive.
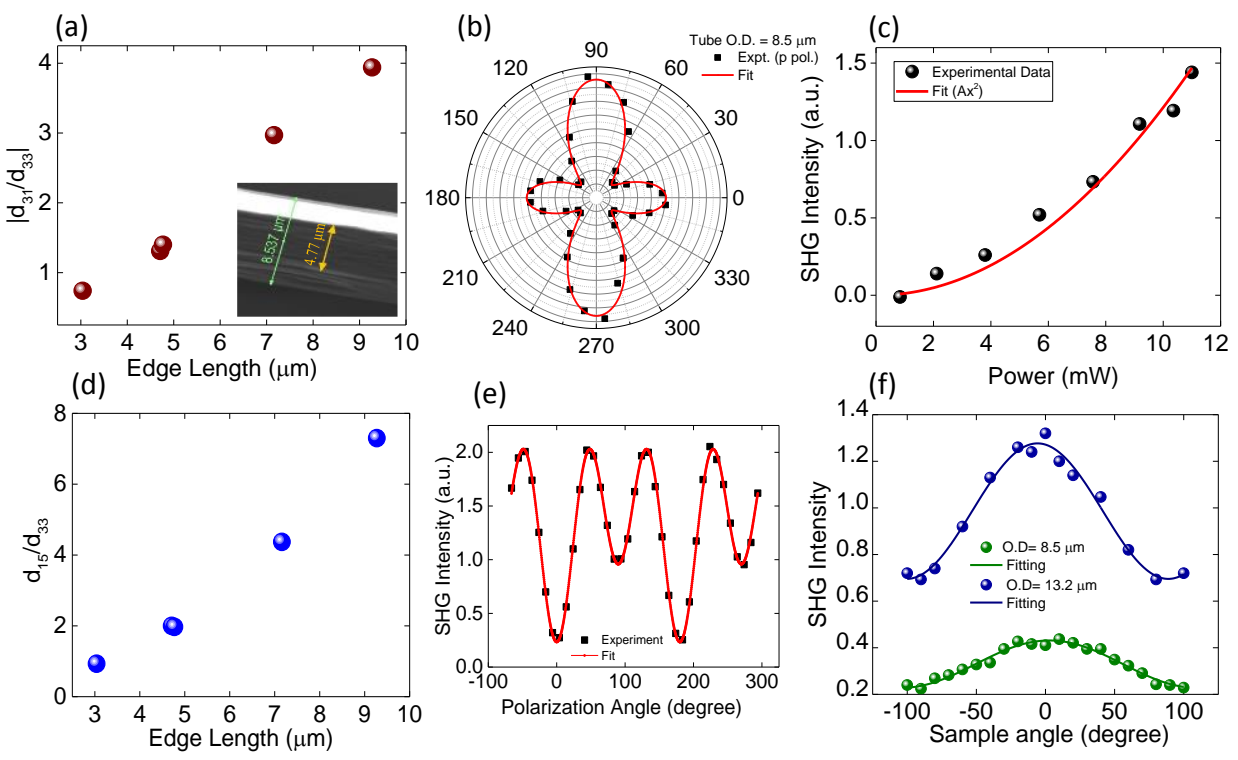

(e)
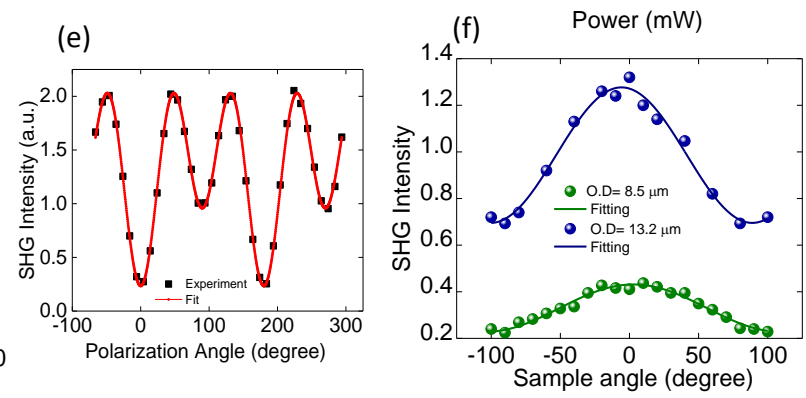

Figure 4.3. (a) Modulus of $d_{31} / d_{33}$ as a function of the edge length of hexagonal FF-MNTs. The inset shows an SEM image of a cross-section of FF-MNT. (b) p-polarization experimental data (black Square) and a fit (red line) using eq ${ }^{\mathrm{n}}$ (4.12). (c) Total SHG intensity as a function of the incident power of the fundamental frequency. The bold line is a fit with square law dependence. (d) $d_{15} / d_{33}$ ratio as a function of the edge length of individual FF-MNTs. (e) Total SHG intensity as a function of the polarization angle of the incident light. Black squares are the experimental results and the red line is a fit to the overall intensity by combining equation (4.11) and (4.12). (f) SHG intensity (s-polarization) as a function of the sample orientation for FFMNTs with O.D. $8.5 \mu \mathrm{m}$ and $13.2 \mu \mathrm{m}$. The solid line is a fit to the square of equation (4.9).

Figure 4.3. (a) and (d) show the modulus of $\left|d_{31} / d_{33}\right|$ and $d_{15} / d_{33}$ ratios, respectively, as a function of the edge length of the hexagonal tubes. Sample fits to the experimental SHG data are shown in Figure 4.3 (b) and (e). Figure. 4.3 (c) shows the overall SHG intensity for a specific FF-MNT as a function of the incident power.

With an increase in the tube diameter, the SHG intensity at $0^{\circ}$ and $180^{\circ}$ in $p$ polarization decreases. As noted above, the overall ratio of $\mathrm{d}_{31} / \mathrm{d}_{33}$ is negative; with increasing tube diameter, the magnitude of this ratio increases. Additionally, the $d_{15} / d_{33}$ 
ratio also increases with the edge length of the hexagonal tubes. The enhancement of the shear piezoresponse has been observed to increase with increasing diameters in FF and other peptide micro/nanotubes using piezoelectric force microscopy. ${ }^{18}$ The number of unit cells in the FF-MNT crystal will dictate the overall SHG intensity. On plotting the $\mathrm{d}_{15} / \mathrm{d}_{33}$ and $d_{31} / d_{33}$ ratio versus edge length as a log-log plot, the slope is close to 2 . With our macrogeometry, where the beam size is bigger than the tube diameter, $\mathrm{I}_{2 \omega}$ scales as the area of the tube with increasing diameter. If we denote the edge length as $l, \mathrm{I}_{2 \omega}$ is proportional to $l^{4}$ and the $\mathrm{d}$ coefficients are proportional to $l^{2}$. A slope of 2 therefore suggests that the transverse coefficients are predominantly changing. Our results are somewhat different from nitrostilbene based organic hexagonal microprisms, ${ }^{39}$ where a micro-SHG setup was used; the spot size of the fundamental light is much smaller than the edge length. Hence, $\mathrm{I}_{2 \omega}$ is proportional to $l^{2}$.

The SHG signal ( $s$-polarization) was also measured by changing the orientation $(\varphi)$ of the sample for two tube diameters, shown in Figure 4.3 (f). The SHG intensity is fit with the square of equation (4.9) by fixing the value of $\Psi$ at $90^{\circ}$. As expected, when $\varphi=0$, the SHG intensity is a maximum. The observed maxima of the two tubes allow a direct comparison of their $d_{15}$ ratios. The tube with O.D $\sim 8.5 \mu \mathrm{m}$ has an edge length of approximately $4 \mu \mathrm{m}$, and the larger O.D. FF-MNT with $13.2 \mu \mathrm{m}$ has an approximate edge length of $9 \mu \mathrm{m}$. A comparison of the peak intensities yields a ratio of $\sim 3$, which is close to the $d_{15}$ ratio of two tubes with similar edge lengths (Figure $4.3(d)$ ). The $d_{15} / d_{33}$ ratio is thus far unknown for FF-MNTs. Our SHG results for the first time show that this anisotropy ratio varies between 1 and 7.5, for the choice of FF-MNT diameters chosen here. 


\subsection{SHG Polarimetry as a Function of Thermal Annealing}

As an attempt to understand the changes in the nonlinear coefficients of FF-MNTs with the diameter of the tubes, we annealed the FF-MNTs at different temperatures. Figure. 4.4 (a) shows the results for a tube with O.D. $\approx 4 \mu \mathrm{m}$. As expected for a small diameter tube, the $p$-polarization data shows strong peaks at $90^{\circ}$ intervals. The sample was annealed at different temperatures for an hour and the SHG intensity was measured from the FF-MNT for both $s$ - and $p$ - polarizations. The overall SHG intensity drops beyond $140{ }^{\circ} \mathrm{C}$. Annealing seems to have the same trend as increasing the diameter of the tubes. The intensity of the two side-bands (at $0^{\circ}$ and $\left.180^{\circ}\right)$ in the $p$-polarization signal decreases.

(a)

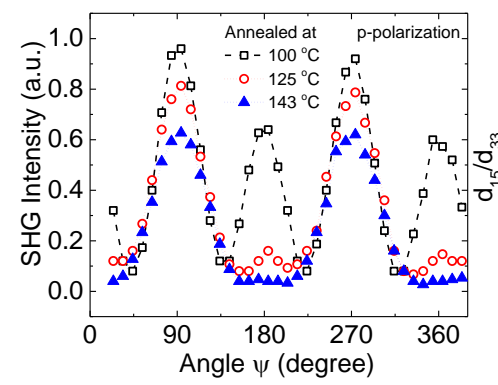

(b)

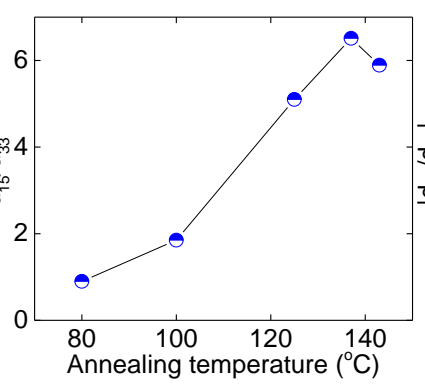

(c)

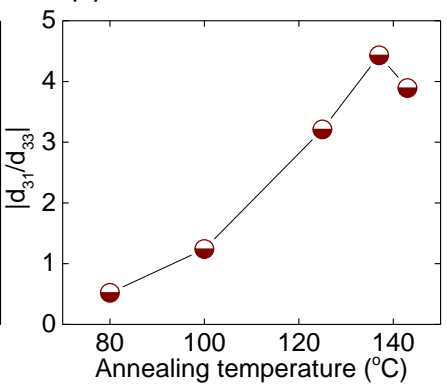

Figure 4.4. (a) SHG response of a FF-MNT (with approximate O.D. of $4 \mu \mathrm{m}$ ) after annealing at temperatures of $100{ }^{\circ} \mathrm{C}$ (square), $125^{\circ} \mathrm{C}$ (circle), and $143{ }^{\circ} \mathrm{C}$ (triangle). (b) $d_{15} / d_{33}$ ratio of the FF-MNT obtained by fits to $\mathrm{eq}^{\mathrm{n}}$ (4.11) and (4.12) as a function of the annealing temperature. (c) $d_{31} / d_{33}$ ratio of the FF-MNT obtained by fits to $\mathrm{eq}^{\mathrm{n}}(4.12)$ as a function of the annealing temperature.

We further observe the ratio of $\mathrm{d}_{15} / \mathrm{d}_{33}$ and $\left|\mathrm{d}_{31} / \mathrm{d}_{33}\right|$ to increase with annealing temperatures till $140^{\circ} \mathrm{C}$. The hexagonal phase changes to the orthorhombic form beyond $\sim 145^{\circ} \mathrm{C}$, and the SHG signal completely disappears as the crystal structure now is centrosymmetric. The similarity in trends of the nonlinear coefficients upon annealing (till $140{ }^{\circ} \mathrm{C}$ ) and upon increasing the diameter raises several questions: (1) are we increasing 
the diameter upon annealing? (2) are there similarities in the change in the crystal structure upon annealing and increasing the diameter? (3) are the changes in the ferroelectric domains similar? It is unlikely that (1) is valid as we would expect some water loss upon annealing, which should actually shrink the diameter of the tubes. In order to obtain further insights, we have conducted XRD and Raman scattering from individual microtubes and FF-MNT powder. We note that the isolated FF-MNTs used in SHG are comprised of several nanotubes. The XRD reveals the structure of single crystallites.

\subsection{XRD Results}

At intermediate annealing temperatures, the XRD data still represents the hexagonal phase but with different crystallite size and water concentration. Upon annealing at $150{ }^{\circ} \mathrm{C}$ the diffraction peaks correspond to the orthorhombic phase.

Table 4.1 reports the Rietveld analysis data. Profile-weighed parameters $\left(R_{w p}\right)$ extracted from Rietveld refinement. The first column lists the annealing temperatures. a, b, $\mathrm{c}$ and $\mathrm{V}$ are the unit cell parameters. $\mathrm{B}_{\text {iso }}$ and $\mathrm{B}_{\mathrm{iso} \_\mathrm{H}}$ are the isotropic displacement parameters of non-hydrogen and hydrogen atoms, respectively. The last column shows the calculated density from the X-ray data.

Table 4.1. Rietveld refinement parameters from FF-MNS.

\begin{tabular}{|c|c|c|c|c|c|c|c|}
\hline $\begin{array}{l}\text { sample } \\
\text { ID }\end{array}$ & $\begin{array}{l}\mathrm{R}_{\mathrm{wp}} \\
(\%)\end{array}$ & $a(\AA)$ & $c(\AA)$ & $\operatorname{Vol}\left(\AA^{3}\right)$ & $B_{\text {iso }}$ & $\mathrm{B}_{\text {iso_H }}$ & $\begin{array}{l}\text { Density }(\mathrm{g} \\
\left.\mathrm{cm}^{-3}\right)\end{array}$ \\
\hline RT & 2.502 & $24.104(2)$ & $5.4460(5)$ & $2740.1(5)$ & $3.5(6)$ & $4.2(7)$ & $1.212(4)$ \\
\hline $80^{\circ} \mathrm{C}$ & 2.477 & $24.106(1)$ & $5.4419(4)$ & 2738.7(4) & $3.7(4)$ & $4.5(5)$ & $1.208(3)$ \\
\hline $135^{\circ} \mathrm{C}$ & 2.606 & $24.118(1)$ & $5.4424(3)$ & $2741.7(3)$ & $4.6(3)$ & $5.5(4)$ & $1.208(2)$ \\
\hline $\begin{array}{l}\text { sample } \\
\text { ID }\end{array}$ & $\begin{array}{l}\mathrm{R}_{\mathrm{wp}} \\
(\%)\end{array}$ & $a(\AA)$ & $\mathrm{b}(\AA)$ & c $(\AA)$ & $\operatorname{Vol}\left(\AA^{3}\right)$ & $\mathrm{B}_{\text {iso }} / \mathrm{B}_{\text {iso_H }}$ & $\begin{array}{l}\text { Density }(\mathrm{g} \\
\left.\mathrm{cm}^{-3}\right)\end{array}$ \\
\hline $150^{\circ} \mathrm{C}$ & 2.369 & $6.164(3)$ & $10.315(6)$ & $23.840(11)$ & $\begin{array}{l}1515.7(1 \\
4)\end{array}$ & $\begin{array}{l}5.1(11) / 6 . \\
1(13)\end{array}$ & $1.290(1)$ \\
\hline
\end{tabular}

The increase in the nonlinear coefficients upon annealing can be explained on the basis of a re-alignment of the ferroelectric domains. As seen in XRD, there may be a slight 
contraction of the individual nanotubes (and loss of water, which is also inferred from Raman scattering, shown in the next section). The loss of water results in a reorganization of the individual nanotubes such that the net effect is an increase in the domain size, and thus a higher dipole moment as schematically shown in Figure. 4.5. Additionally, one may also expect a change in the angular distribution of the dipoles. The incident light samples the ferroelectric domains on the edge of the hexagonal microtube. Enhanced $d_{15} / d_{33}$ and $\left|d_{31} / d_{33}\right|$ ratios with annealing temperatures therefore reflect an increase in the ferroelectric domain size due to a reorganization of the nanotubes and a favorable alignment of the dipole moment of the domains upon loss of water.

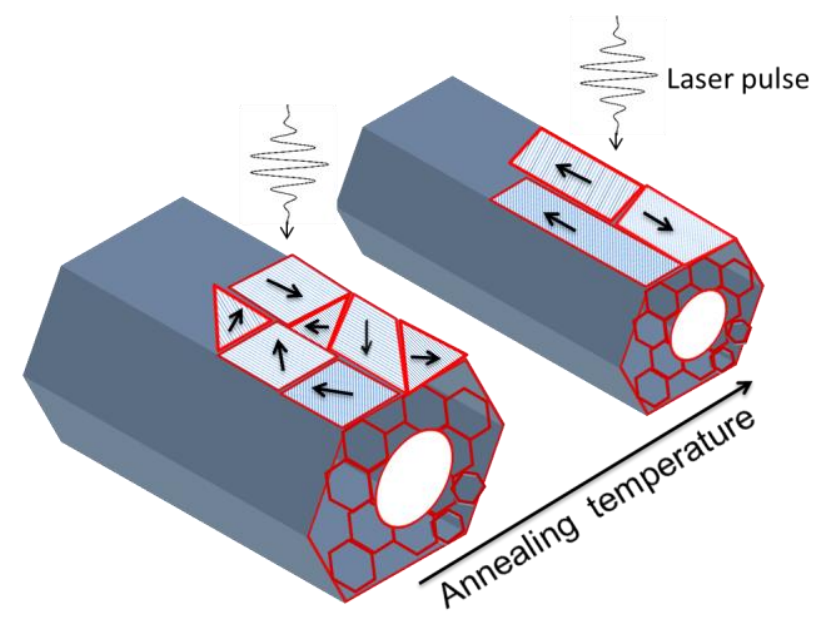

Figure 4.5. A schematic of the change in the domain and crystallite size as a function of the annealing temperature.

\subsection{Raman Scattering Studies}

As a further check for structural changes, we have conducted Raman scattering studies from individual FF-MNTs with varying diameters and as a function of annealing temperature. Since meaningful XRD data from individual FF-MNTs cannot be obtained, direct structural proof from individual tubes is not possible. Figure. 4.6 (a) compares the experimental Raman spectrum of FF-MNT with the calculated spectrum of a FF molecule. 
Figure 4.6 (b) is a sketch of a macrocycle of 6 FF units with water molecules in the hydrophilic channels of the diphenylalanine matrix. This model predicts a more accurate analysis of the XRD data. ${ }^{40}$ The $1000 \mathrm{~cm}^{-1}$ Raman peak is the strongest in FF and FFMNT, which arises from a ring deformation mode. Some of the vibration modes from FFMNTS has been described in Chapter 3. The vibrational bands in the $1200-1330 \mathrm{~cm}^{-1}$ range are typically from amide II vibrations. Details of the displacement pattern of the vibrational modes of FF are found in Ref. $\left[{ }^{41}\right]$. It is not surprising that there are differences in the Raman frequencies between experiment and theory; the experimental data shown is from the self-assembled tubes whereas the theoretical data is only from a diphenylalanine unit. However, the theoretical calculations predict the origin of the displacement pattern of the low frequency vibrational modes, which are greatly affected as a function of the tube diameter.
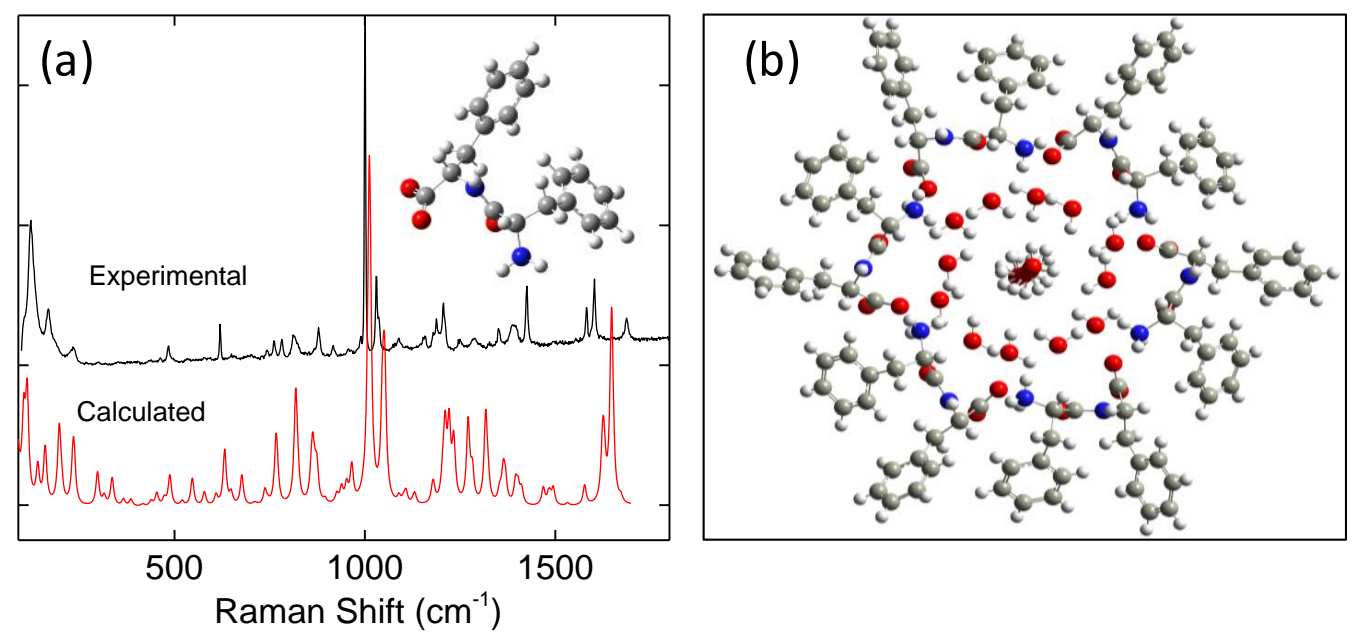

Figure 4.6. (a) Experimental and calculated Raman spectra from FF. The inset shows the structure of the FF molecule. (b) FF macrocycle with water molecules in the core.

The $80 \mathrm{~cm}^{-1}$ peak is predominantly a rocking motion of the amine group and the adjacent phenyl molecule. The $104 \mathrm{~cm}^{-1}$ and $113 \mathrm{~cm}^{-1}$ peaks are IR active with some Raman activity. The $104 \mathrm{~cm}^{-1}$ peak in theory roughly corresponds to the $109 \mathrm{~cm}^{-1}$ peak 
experimentally observed, and originates from an out-of-plane rocking motion of the peptide bond. The $113 \mathrm{~cm}^{-1}$ frequency (observed at $122 \mathrm{~cm}^{-1}$ ) involves a rocking of the peptide bond and a stretching of the oxygen atoms in the carboxyl group.
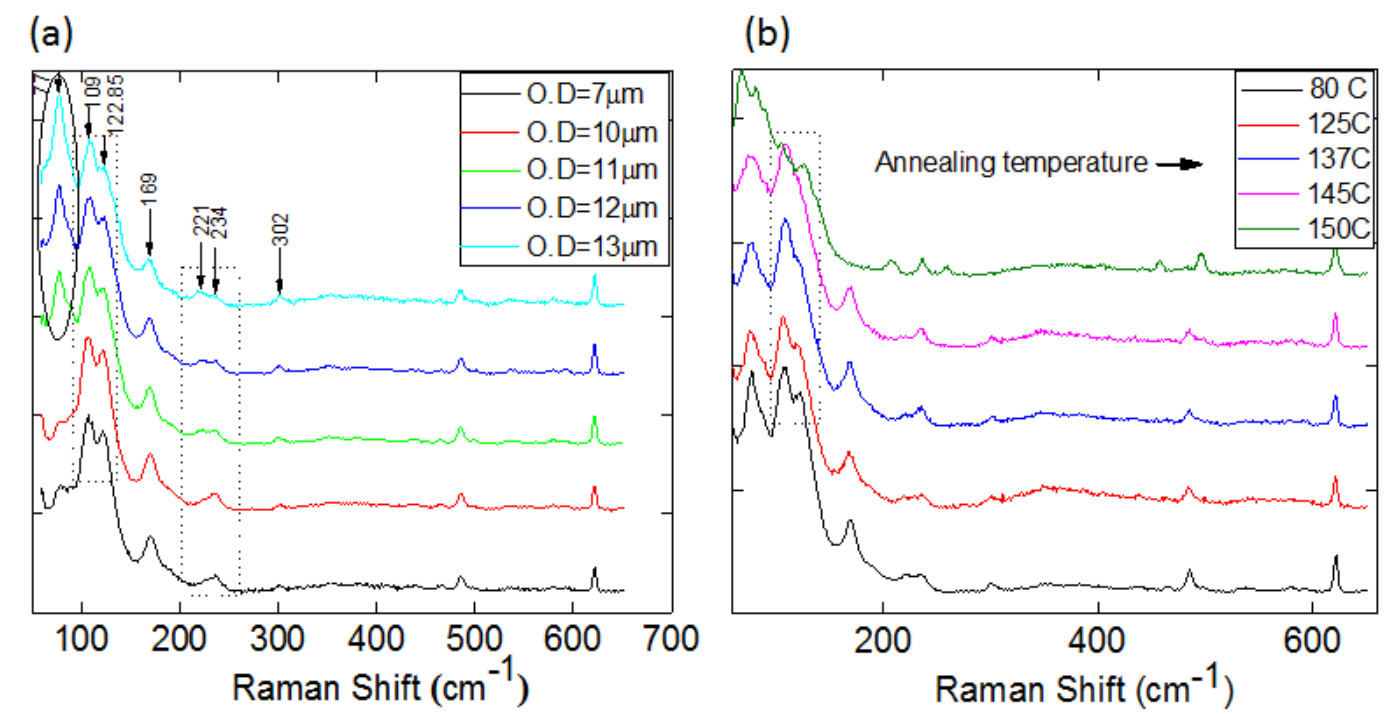

Figure 4.7. (a) Raman spectra as a function of the tube diameter. (c) FF macrocycle with water molecules in the core. (b) Raman spectra of a FF-MNT (O.D. $=11 \mu \mathrm{m})$ as a function of annealing temperature.

Figure 4.7 (a) shows the Raman spectra in the low frequency region of individual FFMNTs with varying O.D. For tubes with O.D. $<10 \mu \mathrm{m}$, the $80 \mathrm{~cm}^{-1}$ peak is not observed. Other subtle changes are observed as a function of the diameter of the tubes. A combined experimental and theoretical work on the high frequency Raman modes of FF shows that water may bind to the FF molecules in the nanochannels, resulting in some splitting of the high energy Raman peaks. ${ }^{42}$ Although we do not see any changes in the high frequency region of the Raman spectrum of individual FF-MNTs with changing tube diameters, the distinct appearance of $80 \mathrm{~cm}^{-1}$ peak beyond a certain tube diameter may be attributed to a coupled motion between FF and water molecules. Since the $80 \mathrm{~cm}^{-1}$ peak is mainly associated with the amine group; coupling with water molecules enhances this feature. For 
small diameters, the amount of water molecules is lower and hence this peak is hardly observed. The low frequency Raman peaks are also seen to change (relative intensity ratio and frequency) with the relative humidity. ${ }^{43}$

Upon annealing, the Raman spectrum from a tube with O.D. $=11 \mu \mathrm{m}$ shows subtle changes in the relative intensity ratio between the $80 \mathrm{~cm}^{-1}$ and the doublet above $100 \mathrm{~cm}^{-1}$ (Figure. 4.7 (b)). There is a slight decrease in the intensity of the $80 \mathrm{~cm}^{-1}$ peak (similar to a smaller diameter tube) suggesting the removal of some water molecules upon annealing, consistent with the XRD data. We note that the spectrum at $150{ }^{\circ} \mathrm{C}$ is from the orthorhombic phase. The doublet at $100 \mathrm{~cm}^{-1}$ mainly involves the motion of the peptide bonds; the ratio between these two peaks changes in a similar fashion both for increasing diameters as well as annealing at higher temperatures. The similarity in the changes in the Raman intensity ratio of the doublet $\left(\sim 100 \mathrm{~cm}^{-1}\right)$ both with diameter and annealing temperatures therefore reflect a change in the crystal structure radially, predominantly affecting the peptide bond.

The Raman spectra both as a function of the tube diameter and annealing temperatures exhibit subtle effects on the role of water. We thus have an indirect proof that the loss of water upon annealing affects the SHG pattern. Based on these observations, one may then conclude that the change in the water content may further affect the crystalline structure and thus the ferroelectric domains as a function of annealing temperatures, resulting in an enhancement of the transverse nonlinear components, namely, $\mathrm{d}_{15} / \mathrm{d}_{33}$ and $\mathrm{d}_{31} / \mathrm{d}_{33}$.

\subsection{Concluding Remarks}

In summary, SHG polarimetry from individual self-assembled peptide micro/nanotubes, allows a precise measurement of the symmetry of the tubes. One of the 
transverse nonlinear components, $d_{31}$, is found to be negative. The ratio of the transverse to the longitudinal nonlinear components, $d_{15} / d_{33}$ and $\left|d_{31} / d_{33}\right|$, increase with the diameter of the tubes, shedding light for the first time on the anisotropy of the nonlinear coefficients in FF-MNTs. SHG polarimetry from FF-MNTs annealed at different annealing temperatures show an enhancement of the nonlinear coefficients $\left(d_{15} / d_{33}\right.$ and $\left.\left|d_{31} / d_{33}\right|\right)$, suggesting a higher alignment of the ferroelectric domains upon increasing annealing temperatures. XRD further suggests a shrinking of the tube diameter and loss of water upon annealing. Raman scattering results also show a subtle effect of water in the low frequency region of the spectrum. The increase in the nonlinear coefficients with the microtube diameter in SHG is related to the number of unit cells per unit volume and the changes in the SHG coefficients with annealing temperatures reflect a slight shrinking of the tube diameter followed by a re-arrangement of ferroelectric domains, enhancing the dipole moment of the system. SHG polarimetry from bio-organic nanostructures proves to be a powerful tool for obtaining structure-property relationships. Furthermore, the biocompatible and rigid nature of peptide micro/nanostructures opens up a sensitive tool for integrating nanophotonics with biosensing activities. 


\section{Functionalization of Peptide Nanostructures with Nanoparticles}

Previous chapters shed light into the easy and low-cost synthesis process and the structural flexibility of FF-MNS nanostructures. After gathering knowledge of the structural properties of FF-MNS in the previous chapters, we focused on studying the interaction of FF-MNS with nanomaterials for different applications. The first part of this chapter focuses on the interaction of FF-MNS with Ag and Au nanoparticles and their application to SERS. The second part of this chapter focuses on the interaction mechanism of CFO with FF-MNS for magnetic and electronic applications.

Nanomaterials with their unique structural, optical, and magnetic properties have a wide range of applications in optoelectronics, aerospace engineering, and healthcare. ${ }^{44}$ The $21^{\text {st }}$ century revolution in biotechnology is strongly linked to the discovery of new functional materials, which are based on biomolecular nanostructures and inorganic nanoparticles. To sustain the recent trends in nano-biotechnology and for furthering the field, it is thus imperative to understand the different mechanisms of interaction at the bionano interface. ${ }^{45-47}$ Protein adsorption and their unfolding properties strongly depend upon the size of the nanoparticles to which they attach. ${ }^{48}$ The phenomenon of cooperativity in proteins due to interacting nanoparticles is another aspect important to biomedical research; the formation of amyloid structures may either be beneficial or harmful. ${ }^{49}$ Similarly, the strength of the interaction between conjugated polymers and peptide nanostructures depends on the backbone conformation of the polymer. ${ }^{50}$ 


\subsection{Functionalization of FF-MNS with Ag and Au Nanoparticles}

\subsubsection{Introduction}

SERS has received a lot of attention in the scientific community due to its huge potential application in different areas of sensitive detection..$^{51-53}$ It is a non-destructive technique, which provides unique fingerprints for molecular identification, leading to very specific and sensitive chemical sensors that can be readily used in forensic, food, and medical analysis. ${ }^{54}$ The development of SERS-active surfaces is mainly based on the organization of $\mathrm{Ag}$ or $\mathrm{Au}$ nanoparticles (AgNps or AuNps) on different substrates such as polymers and paper. ${ }^{55-57}$ There have been recent reports of various plasmonic substrates that allow wavelength independent SERS. ${ }^{58-59}$ The scattering enhancement primarily arises from the local electromagnetic field of surface plasmons, excited by an incident laser light. A second mechanism involves a dynamical charge transfer between the nanoparticles and the molecules involved. Although the latter chemical contribution cannot explain the large Raman intensity enhancement alone, this contribution is an important factor for chemisorbed molecules.

Until now, there are very few works in the literature that have used FF to support SERS active substrates. ${ }^{60-61}$ In one example, FF-MNS was used as a SERS substrate by sputtering gold on top of the assembled nanoflakes. ${ }^{62}$ Herein, we report the spontaneous self-assembly and organization of AgNps or AuNps onto FF-MNS. The SERS activity is monitored from the two crystalline structures of FF-MNS: hexagonal $\left(P 6_{1}\right)$ and orthorhombic $\left(P 22_{1} 2_{1}\right)$. The obtained material was characterized by SEM and TEM, UV-Vis absorption, FTIR, and Raman spectroscopy. Our results show that the hexagonal FF-MNS (hex) and the orthorhombic FF-MNS (ort) serve as SERS templates by the attachment of AgNps and 
AuNps. Rhodamine B $(\mathrm{RhB})$ or rhodamine $6 \mathrm{G}(\mathrm{R} 6 \mathrm{G})$ were used as molecular probes in the micro to nano - molar concentration range for the determination of SERS effect. Firstprinciples DFT calculations of the Raman spectrum using the fundamental unit for hex and ort FF-MNS show a higher Raman intensity from the R6G molecule attached to ort FFMNS due to a more intense energetic chemisorption of R6G in the ort phase compared to the hex phase. FF-MNS with AuNps offer the usage of a longer wavelength excitation, 785 $\mathrm{nm}$, and allowing Raman measurements from both hex and ort FF-MNS. The experimental SERS intensity of R6G ( hundred nanomolar concentration) is found to be higher for ort FF-MNS compared to hex FF-MNS, with an observed enhancement factor of $\sim 10^{5}$, which corroborate our DFT results.

\subsubsection{Synthesis of AgNps+FF-MNSs and AuNps+FF-MNSs}

The synthesis of FF-MNS with Ag/Au Nps was adapted from a general procedure. ${ }^{63}$ First the concentration of AgNps solutions was adjusted to 1.0 O.D. From the stock solution, six different concentrations of AgNps were used to prepare the FF-MNS, as shown in Table 5.1.1. The FF lyophilized powder was dissolved in HFP at $100 \mathrm{mg} \mathrm{mL}^{-1}$. To this HFP solution, a mixture of pure water and AgNps solutions in the ratios above was added with a final concentration of FF-MNS to be $5 \mathrm{mg} \mathrm{mL}^{-1}$.

Table 5.1. Sample names and preparation for FF-MNS with AgNps.

\begin{tabular}{lll}
\hline FF-MNS sample & Water $(\mathrm{mL})$ & $\operatorname{AgNps}(\mathrm{mL})$ \\
\hline 0 & 1.0 & 0.0 \\
1 & 0.9 & 0.1 \\
2 & 0.75 & 0.25 \\
3 & 0.5 & 0.5 \\
4 & 0.25 & 0.75 \\
5 & 0.0 & 1.0 \\
\hline
\end{tabular}


The materials were left for 12 hours for complete assembly and aggregation. After that, the materials were centrifuged, washed once with water, and left to dry in a vacuum chamber at room temperature. The resulting FF-MNS composite at this stage is in the hexagonal phase, $P 6_{1}$. To obtain the orthorhombic phase, the obtained dry material was incubated in an oven at $150{ }^{\circ} \mathrm{C}$ for $90 \mathrm{~min}$ and cooled down to $\mathrm{RT}$, forming at the end the orthorhombic phase, $P 22_{1} 2_{1}$. After that process, the materials were dispersed in water in the concentration of $10 \mathrm{mg} \mathrm{mL}^{-1}$. From this dispersion, the materials were characterized and the films for SERS analysis were prepared by depositing $10 \mu \mathrm{L}$ on a glass slide.

For AuNp+FF-MNS synthesis, FF amorphous powder was dissolved in HFP at a concentration of $100 \mathrm{mg} \mathrm{mL}^{-1}$. A blend of $0.25 \mathrm{~mL}$ water and $0.75 \mathrm{~mL}$ of AuNps was mixed into the FF-MNS+HFP solution. Identical steps for followed as with FF-MNS and AgNps for complete self-assembly and formation of thin films for SERS analysis.

\subsubsection{Results and Discussion}

\subsubsection{SEM and TEM Characterization}

The SEM and TEM images for hex FF-MNS with different amount of AgNps are shown in Figure 5.1. The FF-MNS without Nps, shown in Figure 5.1 (a), highlight the highly porous and fractal surface of FF-MNS. Sample 3 shows the Nps to be dispersed on the surface of the FF-MNS (Figure 5.1 (b)). Furthermore, the Nps are deposited in channels on top of the FF-MNS hexagonal surface. With increasing concentration of Nps (Figure 5.1 (c) and (d)), the FF-MNS structure seems to align and organize the AgNps on their surface.

The TEM images of Sample 5 are shown in Figure 5.1 (e) and (f). These images clearly show the Nps to be aligned on the outer surface of hex FF-MNS, similar to the SEM 
images. From the images it is possible to generate a schematic representation, where FFMNS supports the organization of Nps due to its porous fractal surface.
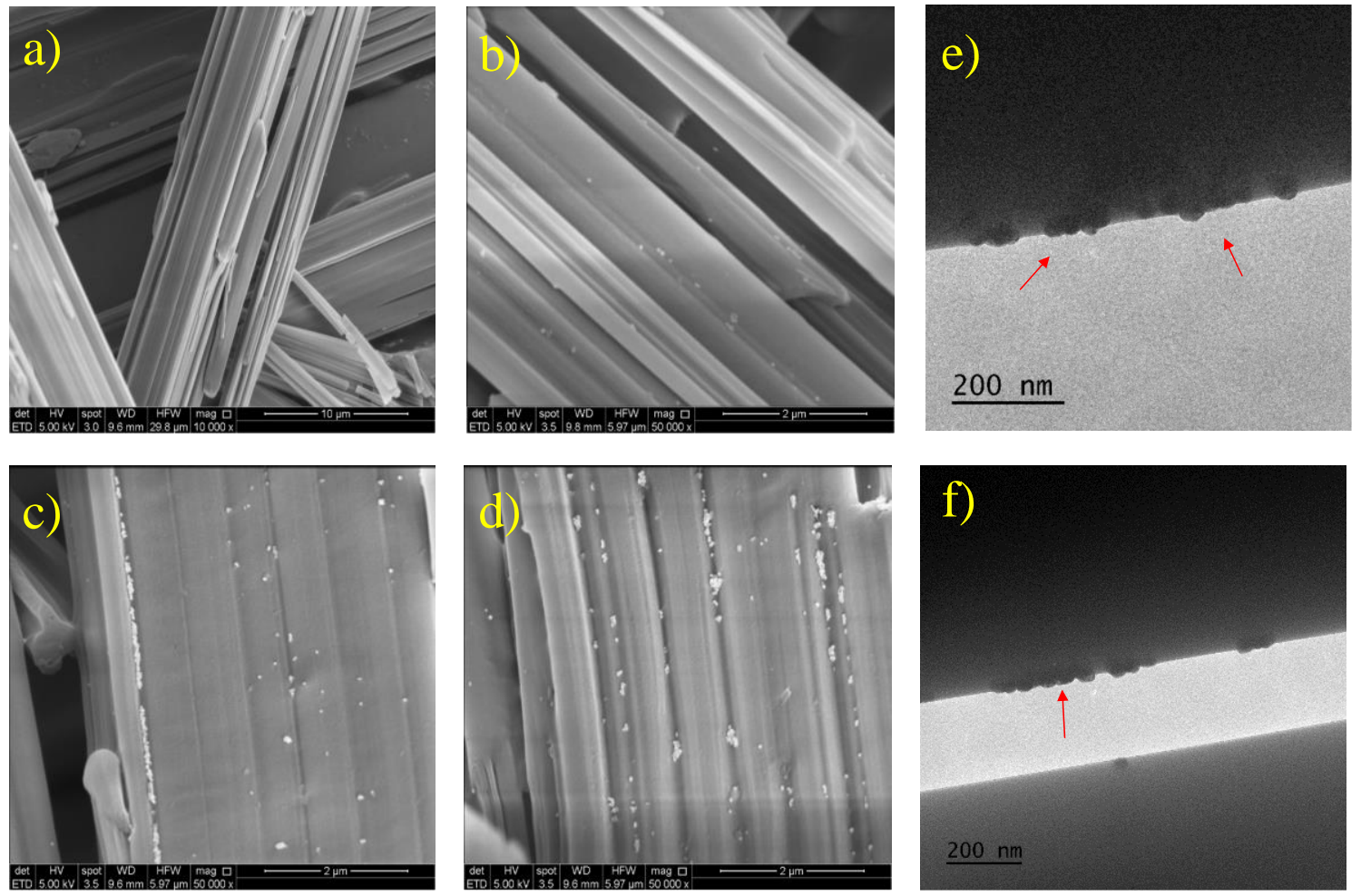

Figure 5.1. SEM images of the FF-MNS composites in the hexagonal $P 6_{1}$ structure with different amount of AgNps. a) Sample 0 without AgNps; b) Sample 3; c) and d) are from sample 5. e) and f) TEM images of Sample 5 shows the AgNps to aggregate on the hexagonal surface.

As mentioned earlier, thermal treatment of the hex phase is converted irreversibly to the orthorhombic structure P22 $22_{1} .{ }^{64-66}$ SEM/TEM images from this phase show clear difference from the hex phase. Figure 5.2 illustrates the very compact structure of ort FFMNS, either as lamellae or a continuous material formed by small plaques. Figure 5.2 (a) shows the ort FF-MNS without AgNps; Figure 5.2 (b) and (c) show the presence of AgNps on top of the structure. It seems that there are fewer Nps on the surface in ort FF-MNS on comparing the SEM images of the hex and the ort phase. The TEM results (Figure 5.2 (d), 
(e)and (f) clearly show that the AgNps reside mainly in the inner core of the ort structure. The thermal treatment during the phase change facilitates migration and dispersion of Nps into the inner channels of the micro/nano tubes. In contrast to the hex phase, where the AgNps aggregate on the surface (within the thinner fibrils), the AgNps are more uniformly distributed throughout the ort FF-MNS tubes, which may enhance the formation of the so called "hot spots".
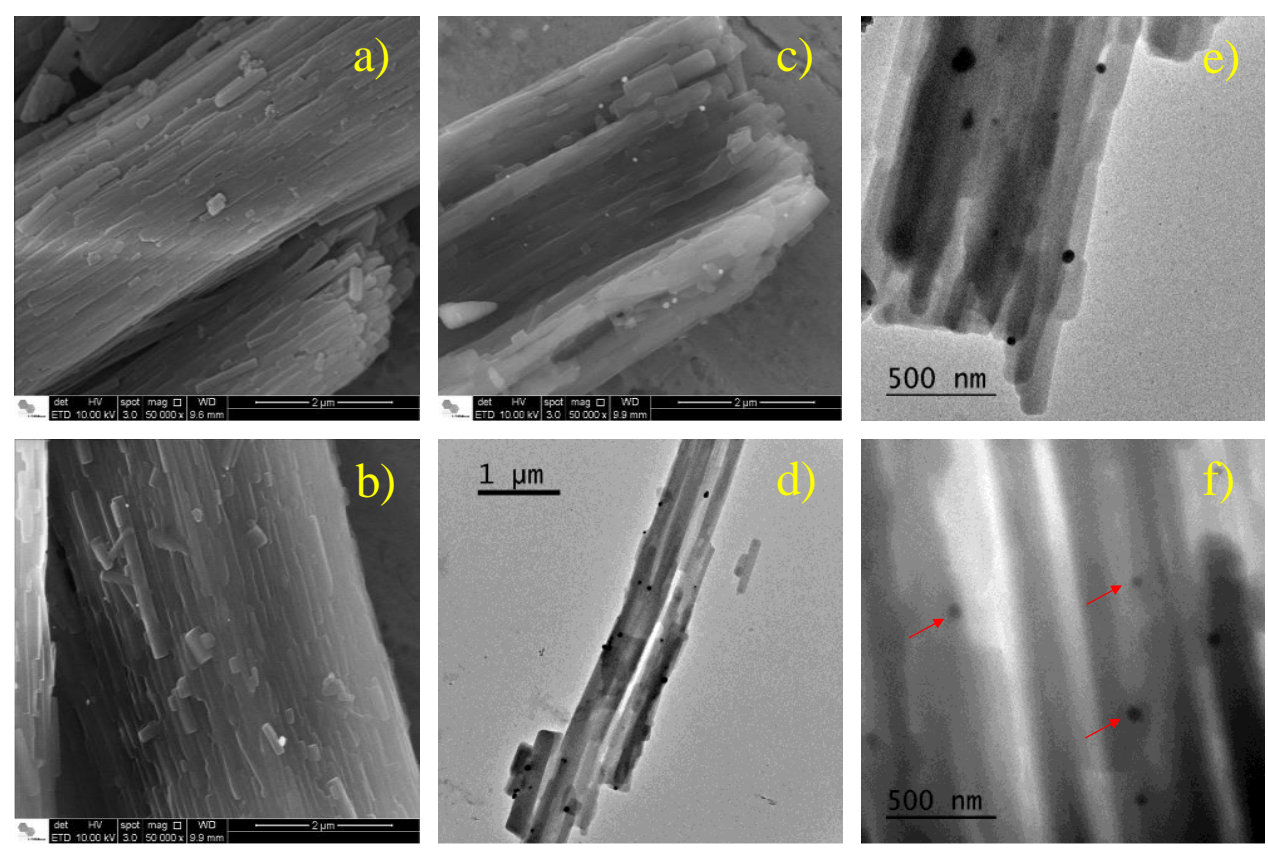

Figure 5.2. SEM images of the FF-MNS composites in the orthorhombic $P 22_{1} 2_{1}$ structure with different amount of AgNps. a) Sample 0 without AgNps; b) Sample 3; c) Sample 5. d), e) and f) are TEM images of Sample 5, showing the AgNps dispersed inside the orthorhombic structure.

Figure 5.3 (a) and (b) shows the SEM images of FF-MNS bonded with AuNps in hex and ort phase. The distributions of the AuNPs on the surface of the nanotubes (hex and ort phases) are similar to the distribution of AgNps on the surface of the nanotubes. AuNps aggregate in hex phase whereas its get well distributed in ort phase. The microscopy results suggest that the metal Nps do not change the structure of FF-MNS. The AgNps decorated hex structure shows the formation of a nanocomposite with the same morphology and 
structure as the non-modified micro-nanostructure. To confirm that the Nps do not alter the chemical structure of FF-MNSs after thermal treatment, UV-Vis and FTIR measurements were performed.

(a)

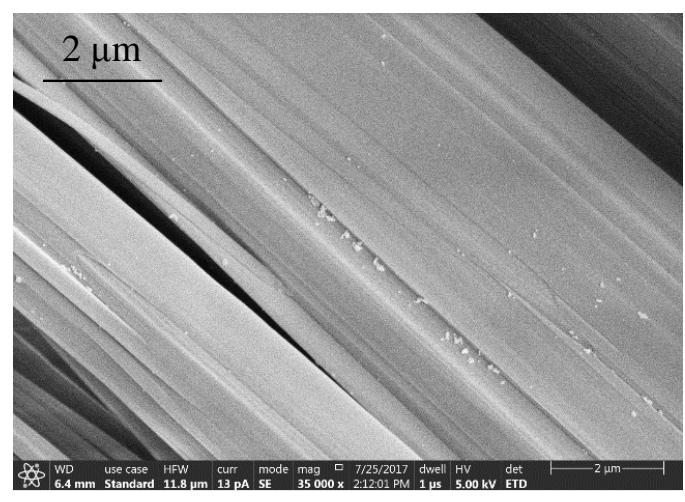

(b)

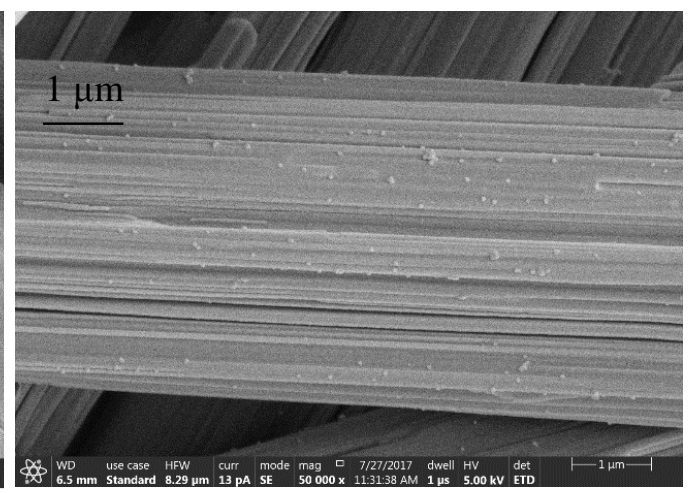

Figure 5.3. SEM images of the FF-MNS composites with AuNps (a) hex phase, and (b) orthorhombic phase.

\subsubsection{UV-VIS and FTIR Characterization}

UV-Vis spectroscopy was used to track the plasmon band since the formation of agglomerates of Nps such as those observed for the hex material would shift the plasmon absorption band to the infrared. ${ }^{67}$ The AgNps, with diameter of approximately $40 \mathrm{~nm}$, dispersed in solution have a very intense absorption at $416 \mathrm{~nm},{ }^{68}$ as seen in Figure 5.4 (ab) (black line). FF-MNS without Nps have a very small absorption in the visible range and an intense absorption due to the aromatic rings and amide groups in the UV region (Figure 5.4 (a-b), red line) which absorbs strongly below $300 \mathrm{~nm} \cdot{ }^{69-70}$

In the presence of the Nps in FF-MNS, there is a color change from white to pale yellow, indicative of absorption by AgNps. In the ort phase a small shoulder at $416 \mathrm{~nm}$ is seen, indicative that the Nps are not aggregated (Figure 5.4 (b) (blue line)). This result agrees with the SEM and TEM images shown previously (Figure 5.2), where the Nps do not aggregate but are well dispersed in the peptide matrix for the ort phase. The spectrum 
for hex FF-MNS also agrees with the SEM and TEM images. The formation of a continuous absorption band starting at $350 \mathrm{~nm}$ until $800 \mathrm{~nm}$ is characteristic of $\mathrm{Np}$ aggregates or agglomerates. ${ }^{67}$ The UV-Vis results suggest that one should observe a higher SERS signal for the ort FF-MNS sample compared to hex FF-MNS due to a more uniform distribution of non-aggregated metal Nps within the FF-MNS framework.

FTIR spectra were further obtained to assess possible changes in the FF molecules and its secondary structure. The FTIR spectrum is very sensitive to structural variations of selfassembled peptides since small changes in its secondary structures results in a very different spectral fingerprint. Both ort and hex FF-MNS have a well described FTIR spectrum; differences due to interactions with $\mathrm{AgNps}$ should result in variations. Figure 5.4 (c) and (d) show the amide group vibrations between $1500-1700 \mathrm{~cm}^{-1}$, characteristic of the hex and ort structures. ${ }^{69}$ Since the FTIR spectra without and with AgNps are very similar for both phases, it indicates that the AgNps do not change the intermolecular features of the assemblies. 
a)

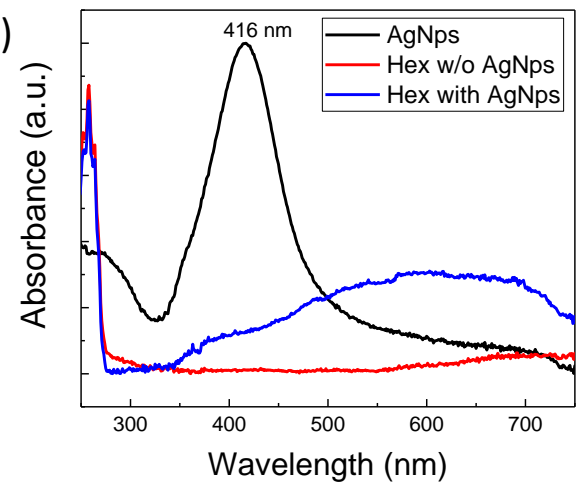

c)

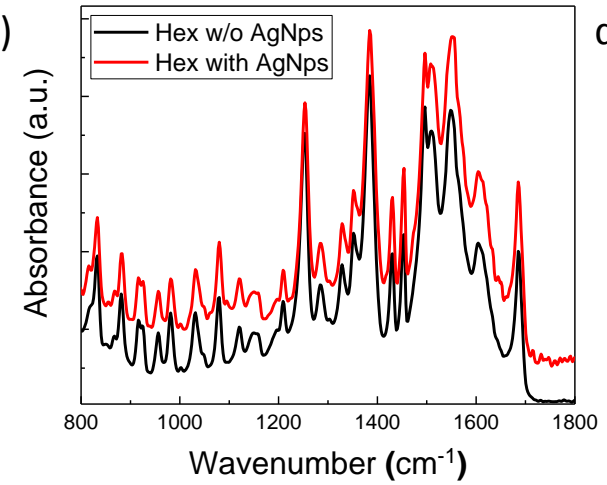

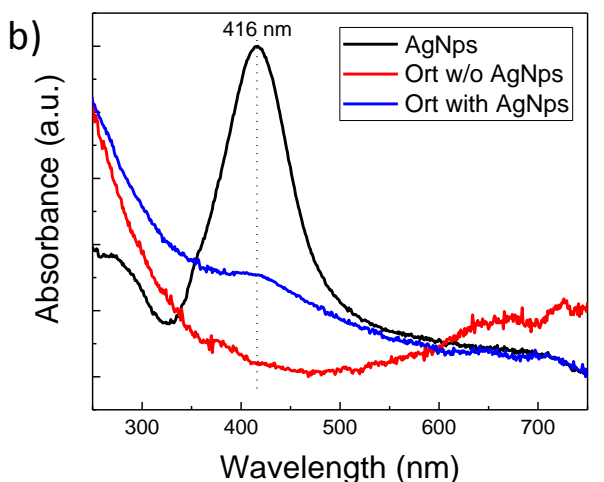

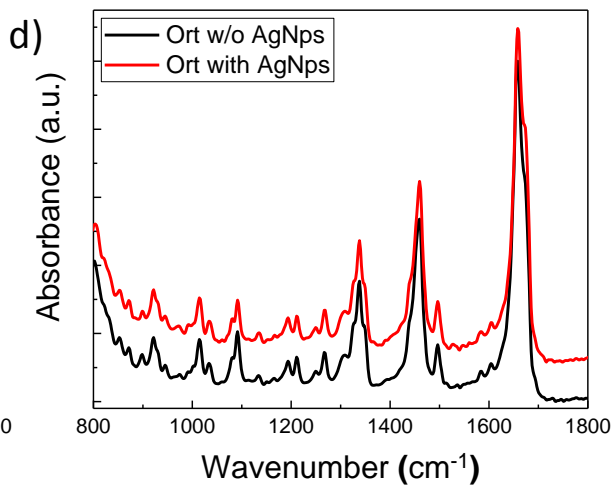

Figure 5.4. UV-Vis of the solutions of AgNps and the FF-MNSs based composites, a) hexagonal FF-MNSs and b) orthorhombic FF-MNSs. FTIR spectra of the peptide assemblies with the c) hexagonal and d) orthorhombic crystal structure. There is no significant difference between the materials without and with AgNps.

\subsubsection{SERS from FF-MNS:AgNps Nanocomposites}

The SERS activity of FF-MNS with AgNps in the two known crystallographic structures was monitored. Both $\mathrm{RhB}$ and $\mathrm{R} 6 \mathrm{G}$ have been used as model molecules for SERS active probe as they do not form any covalent bonds with the metal nanoparticles. We used RhB for SERS studies of FF-MNS with AgNps. During the formation of the nanocomposite film, $5 \mu \mathrm{L}$ of $\mathrm{RhB}$ was added and left to dry for $1 \mathrm{~h}$. All composites were studied by Raman spectroscopy by forming identical films by drop-casting similar volumes of samples on glass slides. The laser power and times were kept constant for all concentrations of AgNps. 

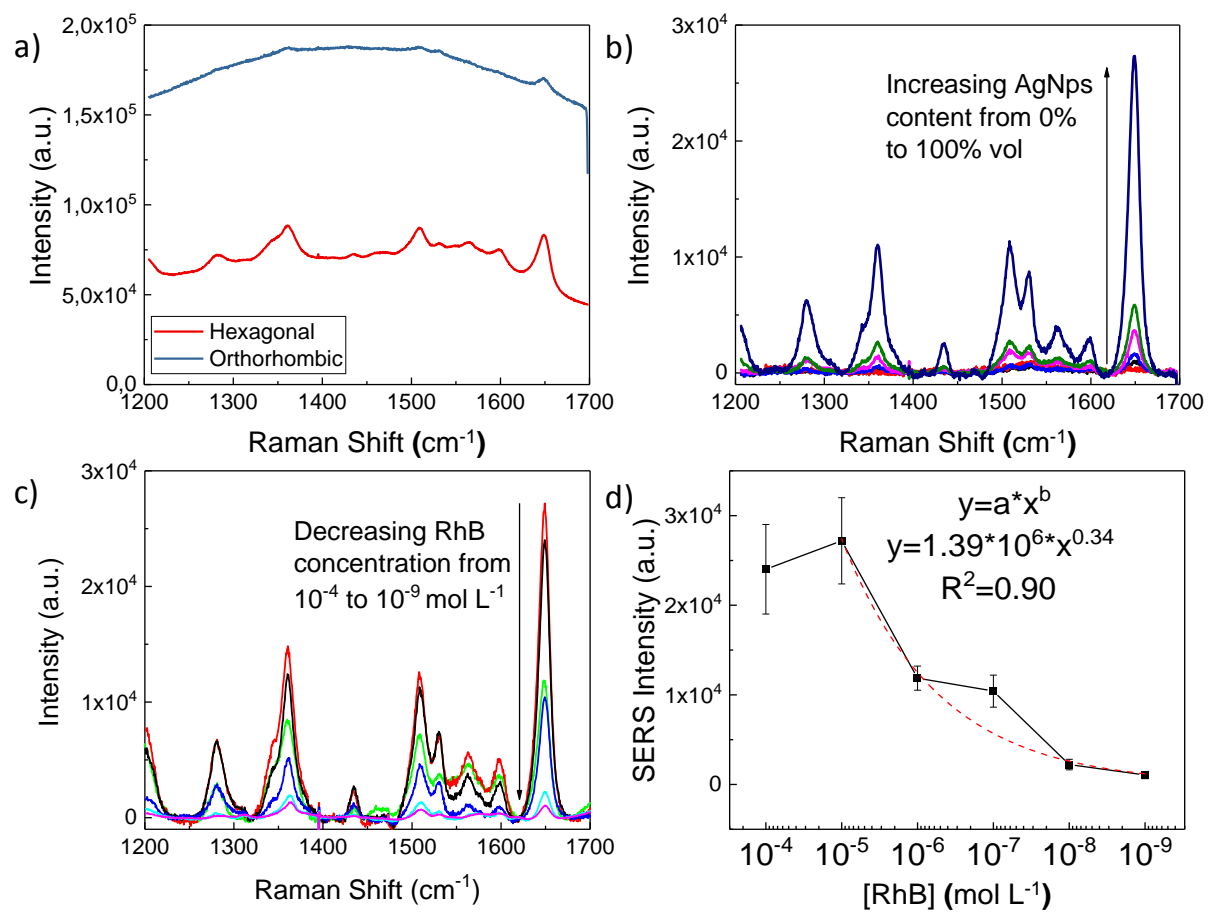

Figure 5.5. a) SERS spectra of RhB from Sample 5 in the hexagonal and orthorhombic structure. The RhB concentration was $10^{-4} \mathrm{~mol} \mathrm{~L}^{-1}$. b) SERS spectra of the hexagonal samples with different amount of AgNps solutions (\%), from 0\% (sample 0) to $100 \%$ (sample 5). c) SERS spectra of sample 5 with different concentrations of RhB, from $10^{-4}$ to $10^{-9} \mathrm{~mol} \mathrm{~L}^{-1}$. d) Calibration curve plot showing the exponential dependence of the concentration of $\mathrm{RhB}$ for the band at $1649 \mathrm{~cm}^{-1}$.

Figure 5.5 summarizes the Raman data using AgNps. Initially the composites with the highest concentration of AgNps (Sample 5) were tested. The fluorescence background from the ort sample is much higher than that of the hex sample as seen in Figure 5.5 (a). Part of that background is attributed to $\mathrm{RhB}$ fluorescence for the high concentration sample $\left(10^{-4} \mathrm{~mol} \mathrm{~L} \mathrm{~L}^{-1}\right)$. The ort sample has a higher optical band gap, contributing to a higher fluorescence background. Semin et al. ${ }^{71}$ observed a strong fluorescence emission for the ort FF-MNs structure with a maximum emission intensity around $500 \mathrm{~nm}$. This fluorescence intensity was 75-times stronger than the intensity for the hex structure. Since the laser wavelength $(\lambda=533 \mathrm{~nm})$ for the SERS measurements is close to the emission maximum of ort FF-MNs, the Raman peaks cannot be tracked from ort FF-MNS. However, 
the Raman spectra from the hex phase could be monitored for various concentrations of $\mathrm{RhB}$ and $\mathrm{AgNps}$ The positions and the assignment of the observed vibrational modes are given in Table 5.2.

Table 5.2. Experimental SERS peaks of RhB and assignment of the bands.

\begin{tabular}{lll}
\hline This work $\left(\mathrm{cm}^{-1}\right)$ & Literature $\left(\mathrm{cm}^{-1}\right)^{72-73}$ & Assignment \\
\hline 1282 & 1280 & C-C bridge-bands stretching \\
1360 & 1356 & Aromatic C-C stretching \\
1434 & 1422 & Xanthene ring stretching \\
1508 & 1506 & Aromatic C-C stretching \\
1565 & 1565 & Aromatic C-C stretching \\
1599 & 1595 & C=C stretching \\
1649 & 1648 & Aromatic C-C stretching \\
\hline
\end{tabular}

Figure 5.5 (b) shows changes in Raman intensity by varying the concentration of AgNps. A maximum enhancement is observed for Sample 5 with the highest concentration of AgNps. The concentration of RhB was also varied as shown in Figure 5.5 (c). A correlation between the SERS intensity and concentration is observed for decreasing the concentration of $\mathrm{RhB}$ from $10^{-4}$ to $10^{-9} \mathrm{~mol} \mathrm{~L}^{-1}$; even for nano molar concentrations, the Raman peaks from RhB are observable. The calculated limit of detection was found to be $0.45 \mathrm{nmol} \mathrm{L} \mathrm{L}^{-1}$ of $\mathrm{RhB}$. The SERS intensity as a function of $\mathrm{RhB}$ concentration shows an exponential profile (Figure5.5 (d)).

The use of AgNps with FF-MNS leaves several questions unanswered. Does the ort phase show any SERS effect? Will the same Raman bands of the molecular probe enhance in the ort and the hex phase? Do the Raman peaks of FF-MNS show any enhancement in the presence of metal Nps? To answer these questions, we carried out first-principles calculations of the vibrational spectrum (in the presence of a metal atom) and a complete 
SERS study using AuNps with FF-MNS. Since R6G has less overlapping Raman peaks with FF-MNS compared to $\mathrm{RhB}$, we used $\mathrm{R} 6 \mathrm{G}$ as the molecular probe for these experiments. Next, we present our first principles DFT calculations of the vibrational spectrum of R6G when attached to the ort and hex units of FF-MNS in the presence of an Ag atom.

\subsubsection{DFT Calculations}

Calculation method and extracting vibrational spectra from the calculation has been described in Chapter 2, Section 2.5. The optimized structures for R6G attached to the FF:Ag (or Au) units were used for obtaining the vibrational spectrum. We note that Raman activities are obtained from the calculations. The absence of any imaginary frequencies in our calculations confirms stable structures for the molecules.

Figure 5.6 (a) and (b) shows the hex and ort FF unit, respectively. Figure 5.6 (c) shows

the structure of R6G used for the calculations. ${ }^{74}$ To determine the appropriate binding energy of the $\mathrm{Ag}$ atom with the FF unit, the optimized structure (with minimum energy) was determined by varying the position of the $\mathrm{Ag}$ atom with respect to the FF molecule. For such structures, the $\mathrm{Ag}$ atom interacts concomitantly with the $\mathrm{C}=\mathrm{O}$ and $\mathrm{N}-\mathrm{H}$ groups of the amide bond in both hex and ort FF unit. Dissociation energies (DE) of FF molecules were calculated using the optimized structures. $\mathrm{DE}=($ Optimized energy of the metal FF complex) - (optimized energy of Ag + optimized structure of FF). ${ }^{75}$ Calculated DE values tabulated in Table 5.3 confirm that the chemical adhesion of the Ag atom to the FF unit is higher for the ort structure compared to the hex FF unit. 
Table 5.3. Dissociation energy of the Ag atom with respect to the FF unit.

\begin{tabular}{lll}
\hline Molecule & $\begin{array}{l}\text { Optimized Energy } \\
(\text { Hartree })\end{array}$ & $\begin{array}{l}\text { Dissociation energy } \\
\left(\mathrm{kcal} \mathrm{mol}^{-1}\right)\end{array}$ \\
\hline hex FF & -1019.381711 & \\
$\mathrm{Ag}$ & -5148.411933 & \\
hex FF+Ag & -6167.937279 & -90.13 \\
ort FF & -944.025863 & \\
$\mathrm{Ag}$ & -5148.411933 & \\
ort FF $+\mathrm{Ag}$ & -6092.610985 & -108.67 \\
\hline
\end{tabular}

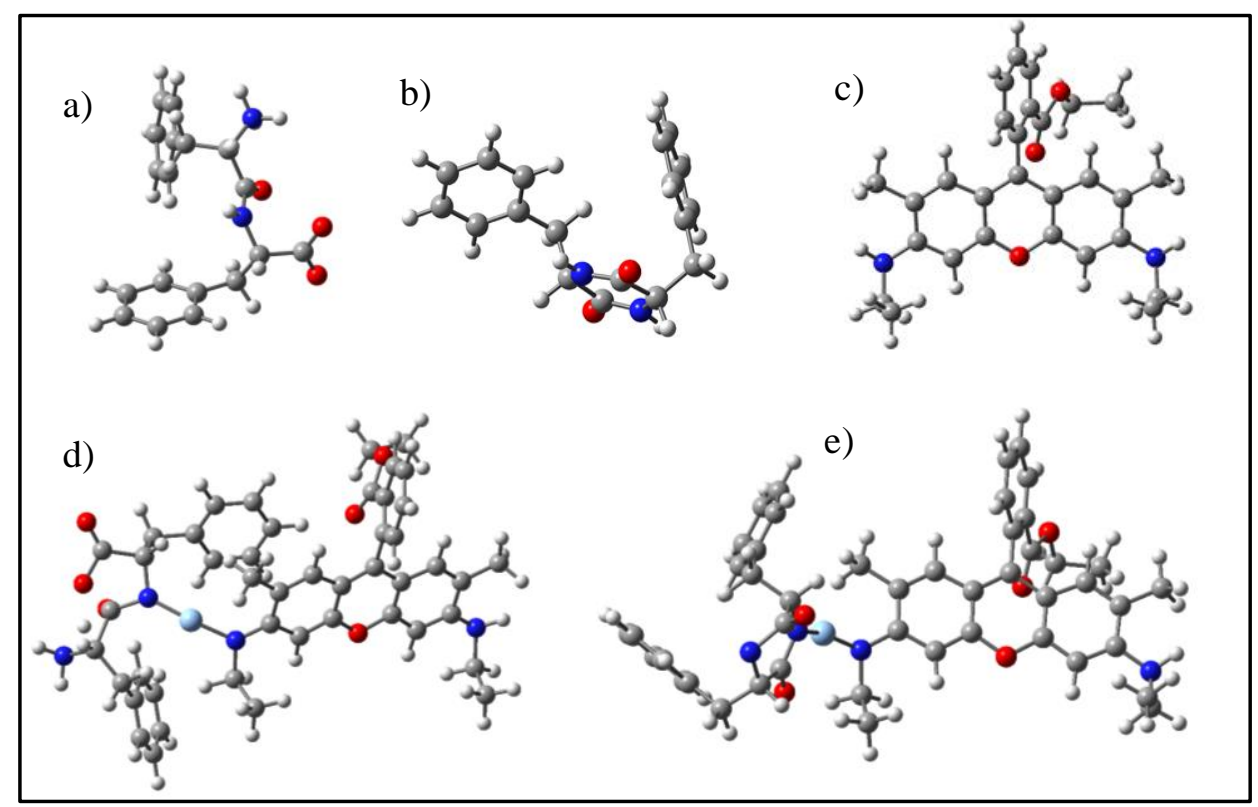

Figure 5.6. a) Hexagonal (hex) FF unit; b) Orthorhombic (ort) FF unit; c) R6G molecule; d) hex $\mathrm{FF}+\mathrm{Ag}$ with $\mathrm{R} 6 \mathrm{G}$; e) ort $\mathrm{FF}+\mathrm{Ag}$ with $\mathrm{R} 6 \mathrm{G}$.

The binding of the $\mathrm{Ag}$ atom to the $\mathrm{N}$ atom of $\mathrm{R} 6 \mathrm{G}$ yields the lowest energy configuration as shown in Figure 5.6 (d) and (e). Ag-N bonding in the Raman spectrum has also been observed experimentally. ${ }^{76}$ Comparing the DE from the two FF conformations, one would expect a higher SERS from the ort phase, predominantly due to an enhanced chemisorption process. Experimentally, the electromagnetic field enhancement will further depend upon the organization of $\mathrm{Ag} / \mathrm{Au}$ nanoparticles on FFMNS. We calculated the Raman spectrum of a R6G molecule, the two FF units with and 
without $\mathrm{Ag}$, and $\mathrm{FF}+\mathrm{Ag}+\mathrm{R} 6 \mathrm{G}$ (for both hex and ort structures). We note that the Raman spectra of R6G and RhB (as used for SERS measurements for AgNps) are slightly different.

In Figure 5.7 (a) and (b), we plot the calculated Raman cross-section in the 1200-1600 $\mathrm{cm}^{-1}$ range from $\mathrm{FF}+\mathrm{Ag}+\mathrm{R} 6 \mathrm{G}$ for both hex and ort units. Additionally, the calculated spectra of FF, FF+Ag, and R6G are shown with their respective Raman cross-sections. For the ort FF unit and ort FF+Ag (Figure 5.7 (b)), there are some shifts seen in the Raman peak positions but qualitatively they are similar. Additionally, there is a slight intensity enhancement; the peaks in the $1400 \mathrm{~cm}^{-1}$ region, which corresponds to the aromatic ring vibrations, show an enhancement by a factor of 2 (from approximately $6 \times 10^{-31}$ to $12 \times 10^{-31}$ $\mathrm{cm}^{2} / \mathrm{sr}$ ). In comparison, the Raman intensity from R6G is one order of magnitude higher compared to FF and FF+Ag. The $1286 \mathrm{~cm}^{-1}$ and $1445 \mathrm{~cm}^{-1}$ Raman peaks seen in the composite sample (the green curve), are enhanced by factors of 60 and 10, respectively with respect to only R6G (the red curve). The $1286 \mathrm{~cm}^{-1}$ mode corresponds to the asymmetric stretching of $\mathrm{COO}^{-}$group along with a symmetric stretching of ethylamine group in $\mathrm{R} 6 \mathrm{G}$, and the $1445 \mathrm{~cm}^{-1}$ mode is assigned to the breathing mode of the phenyl ring (or xanthene ring).

Similarly, for hex FF (Figure 5.7 (a)), the complex with Ag and R6G identifies signatures that originate only from R6G. The $1421 \mathrm{~cm}^{-1}$ vibration of R6G, which corresponds to the breathing motion of the phenyl rings, shows a maximum enhancement when bound to the hex unit and Ag. The Raman peaks corresponding to FF show a higher enhancement with Ag in the hex phase compared to the ort phase. Thus, the two main observations from the calculated spectra of R6G complexes are that the enhancement of different R6G Raman peaks are different, which depend on whether the molecule is 
attached to the hex or ort FF units, and that the overall enhancement in R6G is higher by a factor of two when bound to the FF ort unit compared to the hex unit.

(a)

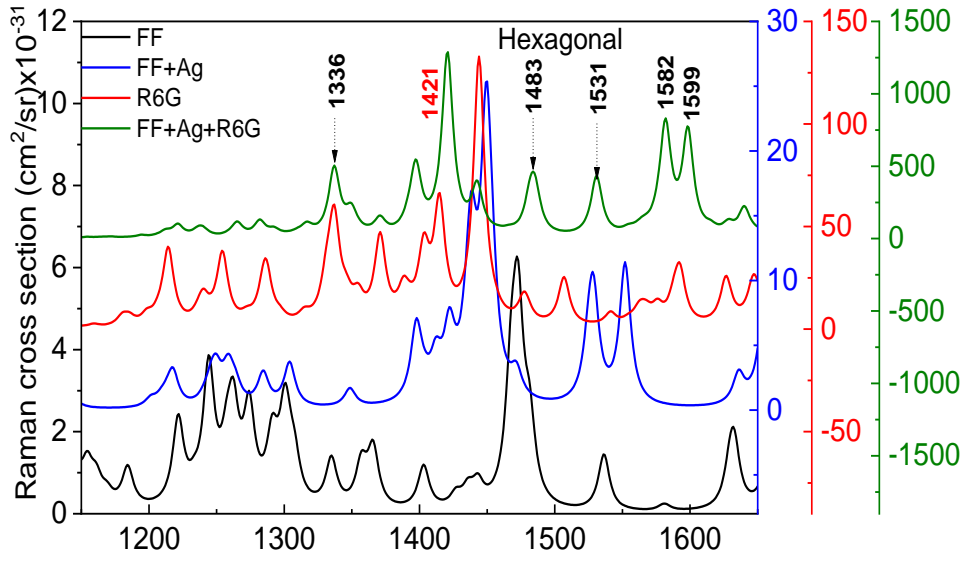

(b) Raman Shift $\left(\mathrm{cm}^{-1}\right)$

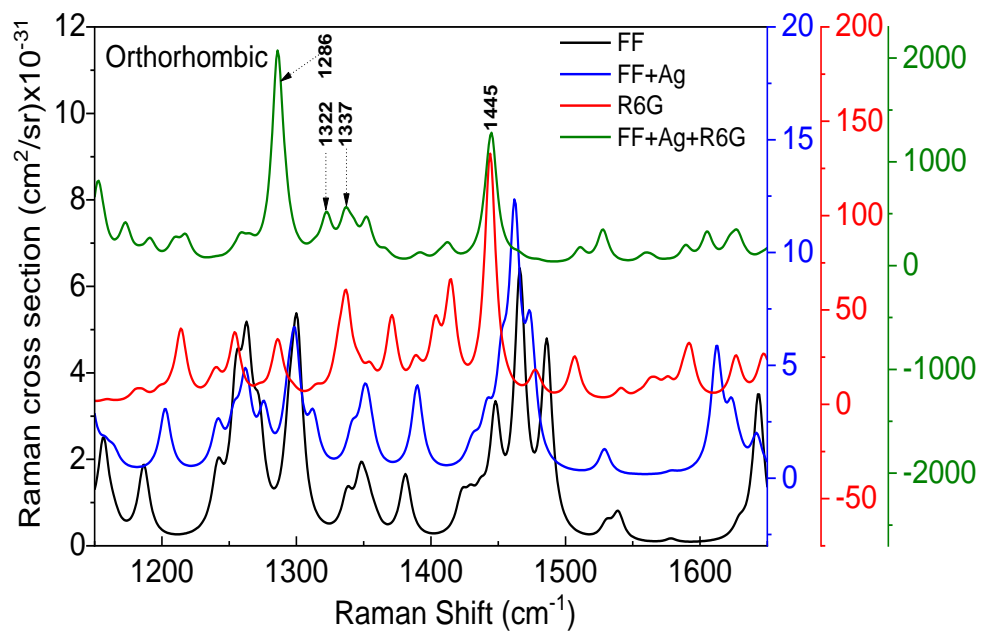

Figure 5.7. Calculated Raman spectra of $F F, F F+A g, R 6 G$ and $F F+A g+R 6 G$ in the a) hexagonal and $b$ ) orthorhombic phase. The spectra are vertically scaled to depict the calculated Raman intensities. The color of the vertical axis corresponds to the color of the Raman spectra. All Raman cross sections are quoted in terms of $\mathrm{cm}^{2} / \mathrm{sr}\left(\times 10^{-31}\right)$.

Since the Raman spectra calculations are from the gas phase, the calculated frequency positions are shifted from the experimental data. Moreover, the calculations take into consideration only one Ag atom and the fundamental unit of FF-MNS. Despite these constraints, there is a clear enhancement of the R6G Raman peaks in the presence of $\mathrm{Ag}$ 
attached to FF. The calculations serve as a model for explaining our experimental data. The ort FF clearly shows a higher binding energy of the $\mathrm{Ag}$ atom to the FF unit and shows a higher enhancement of the R6G Raman peaks compared to hex FF. This suggests that the lack of observation of any Raman peaks from RhB when bound to ort FF-MNS+Ag (as discussed previously, Figure 5.5) is limited mainly due to the luminescence from the ort FF sample. Electronic structure calculations of FF-MNS show slightly higher bandgap energy for the ort phase compared to the hex ${ }^{66}$ Raman scattering measurements from the ort FF phase using the $533 \mathrm{~nm}$ wavelength (as required for observing SERS with Ag) result in a higher fluorescence background, rendering SERS measurements almost impossible. To obtain a complete picture of how FF-MNS substrates serve as templates for SERS, we have conducted Raman measurements using AuNps as well. These measurements were obtained with the $785 \mathrm{~nm}$ laser line, below the optical bandgap energy of FF-MNS.

\subsubsection{SERS from FF-MNS:AuNps}

All SERS measurements were performed with a fixed concentration of AuNps and $10^{-}$ ${ }^{7}$ molar R6G concentration. The ort FF-MNS phase was obtained using the same procedure as with the AgNps. Figure 5.8 shows two representative SERS spectra from R6G on hex and ort FF-MNS+AuNps. One clearly sees the SERS spectrum from the ort phase using the $785 \mathrm{~nm}$ excitation source unlike with the $533 \mathrm{~nm}$ line excitation from FF-MNS+AgNps. In order to estimate the Raman enhancement factors, we compared the SERS spectra from both hex and ort FF-MNS with a higher concentration $\left(10^{-4}\right.$ molar) of R6G film, where the Raman peaks were easily discernible. By normalizing the Raman spectrum with measurement times (and the incident laser power, which was the same in each case), we obtain an enhancement factor of $1.6 \times 10^{5}$ for ort FF-MNS and $0.7 \times 10^{5}$ for hex-FFMNS, 
respectively. We used the formula: $\mathrm{F}_{\mathrm{e}}=\frac{I_{\text {Sample }} C_{\text {ref }}}{I_{\text {ref }} C_{\text {sample }}}$, where $\mathrm{F}_{\mathrm{e}}=$ enhancement factor, $I_{\text {Sample }}=$ SERS intensity of R6G, $I_{r e f}=$ Intensity of R6G, $C_{r e f}=$ concentration of R6G, and $C_{\text {sample }}=$ concentration of R6G in FF-MNs. The amount and the area of the drop casted solution onto quartz substrates were same for both the reference and the SERS sample.

a)

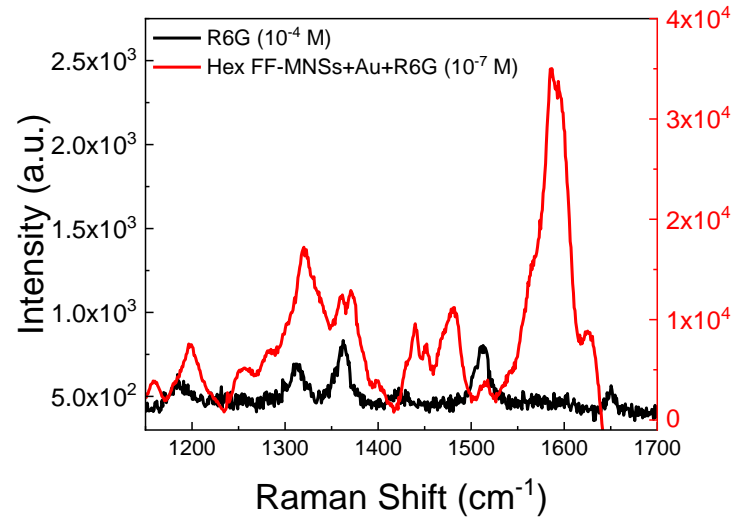

b)

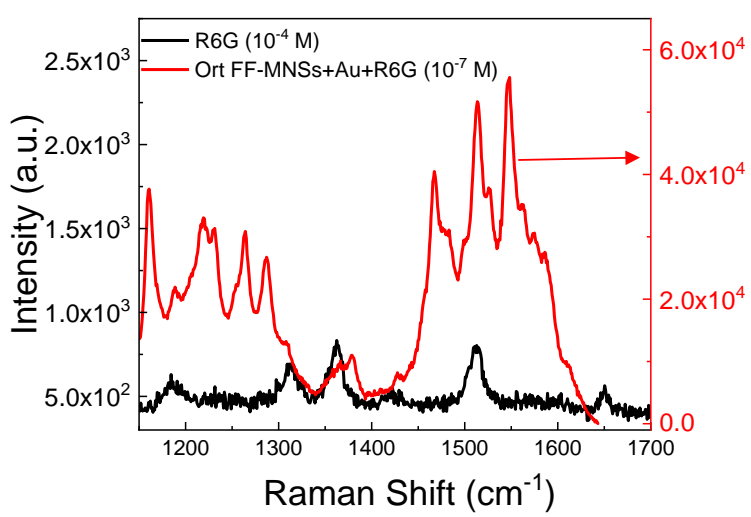

Figure 5.8. Raman spectra of pure R6G $10^{-4} \mathrm{~mol} \mathrm{~L}^{-1}$ (black) and R6G $10^{-7} \mathrm{~mol} \mathrm{~L}^{-1}$ attached to FF-MNS. a) hex FF-MNS+AuNps+R6G; b) ort FF-MNS+AuNps+R6G spectra.

The confocality of the micro-Raman setup ensures that there is hardly any penetration of the laser line into the FF-MNS layer. We measured the SERS effect only from FF-MNS as well as R6G using the same concentration of AuNps concentration as used for the composite sample. This allows us to compare the SERS effect from all samples using the same experimental conditions, and to obtain a better insight into the origin of the vibrational modes. The experimental Raman spectra in the $1200-1600 \mathrm{~cm}^{-1}$ range are plotted for all samples in Figure 5.9. There is some enhancement of the FF-MNS Raman peaks in the presence of AuNps, especially for hex FF-MNS; the $1426 \mathrm{~cm}^{-1}$ Raman peak shows a strong enhancement (Figure 5.9 (a)). The enhancement of the $1426 \mathrm{~cm}^{-1}$ Raman peak in FF-MNS agrees with theory (Figure 5.7 (b)), where the theoretical Raman cross section is enhanced by a factor of 3 (from $6 \times 10^{-31}$ to $20 \times 10^{-31} \mathrm{~cm}^{2} / \mathrm{sr}$ ). 
The R6G film with only AuNps was made with a $10^{-7}$ molar concentration of R6G (similar to the FF-MNS complexes). As expected, a SERS effect is observed from R6G+AuNps. All spectra in Figure 5.9 have been measured with the same laser power and times so that one may compare the actual intensities. The binding of R6G with FFMNS+AuNps results in a very different Raman signature compared to only R6G+AuNp. The $1585 \mathrm{~cm}^{-1}$ and $1596 \mathrm{~cm}^{-1}$ Raman peaks, attributed to the asymmetric stretching of the phenyl rings, are strongly enhanced when R6G is attached to hex FF-MNS. The theoretical peak positions are at $1581 \mathrm{~cm}^{-1}$ and $1598 \mathrm{~cm}^{-1}$, respectively.

There are several other R6G Raman peaks that get enhanced when attached to ort FFMNS+AuNps. These are at 1310, 1219, 1232, 1360, 1512, and $1574 \mathrm{~cm}^{-1}$, which are assigned mainly to the aromatic stretching vibration of R6G molecule. ${ }^{77}$ Theoretically, the Raman peaks are at $1317,1217,1236,1366$, and $1511 \mathrm{~cm}^{-1}$; the $1574 \mathrm{~cm}^{-1}$ peak is not seen in theory. The $1562 \mathrm{~cm}^{-1}$ is assigned to the C-C stretching vibration from the xanthene vibration, and the $1265 \mathrm{~cm}^{-1}$ is attributed to the vibration of the $\mathrm{C}-\mathrm{O}-\mathrm{C}$ bond. The $1479 \mathrm{~cm}^{-}$ ${ }^{1}$ peak (theoretically at $1483 \mathrm{~cm}^{-1}$ ) is from the bending vibration of the ethylamine group in R6G.

Different R6G Raman peaks may get enhanced depending on the choice of the SERS substrate, mainly due to differences in the chemisorption process. ${ }^{78-79}$ The two phases of the FF-MNS yield very different SERS spectra of R6G. Our DFT calculations from the fundamental units shed some light. The metal atom with the ort unit is more positive (suggesting that it gives up more negative charges) compared to the one with the hex unit. Additionally, the monomer units for the hex and ort phases are different (Figure 5.6), resulting in differences in the bond polarization in the vicinity of the attachment of the R6G 
molecules to FF. Since the Raman intensities are governed by the derivative of the electronic polarizability tensor with respect to the normal coordinate of a mode, ${ }^{80}$ it is not surprising that different vibrations of R6G show enhancement when bound to the hex and ort phases of FF-MNS.

One clear observation from our experiments is that the SERS intensity of R6G is higher when FF-MNS+AuNps is used as the substrate compared to using only AuNps. With only AuNps, the SERS signature from R6G is similar to what is seen for the high concentration R6G sample without any metal Nps (Figure 5.8). The SERS results from the FF-MNS composites clearly show that there is a chemical interaction between FF-MNS+AuNps and R6G, which not only enhances the SERS signals but also changes the selection rules for Raman activity.
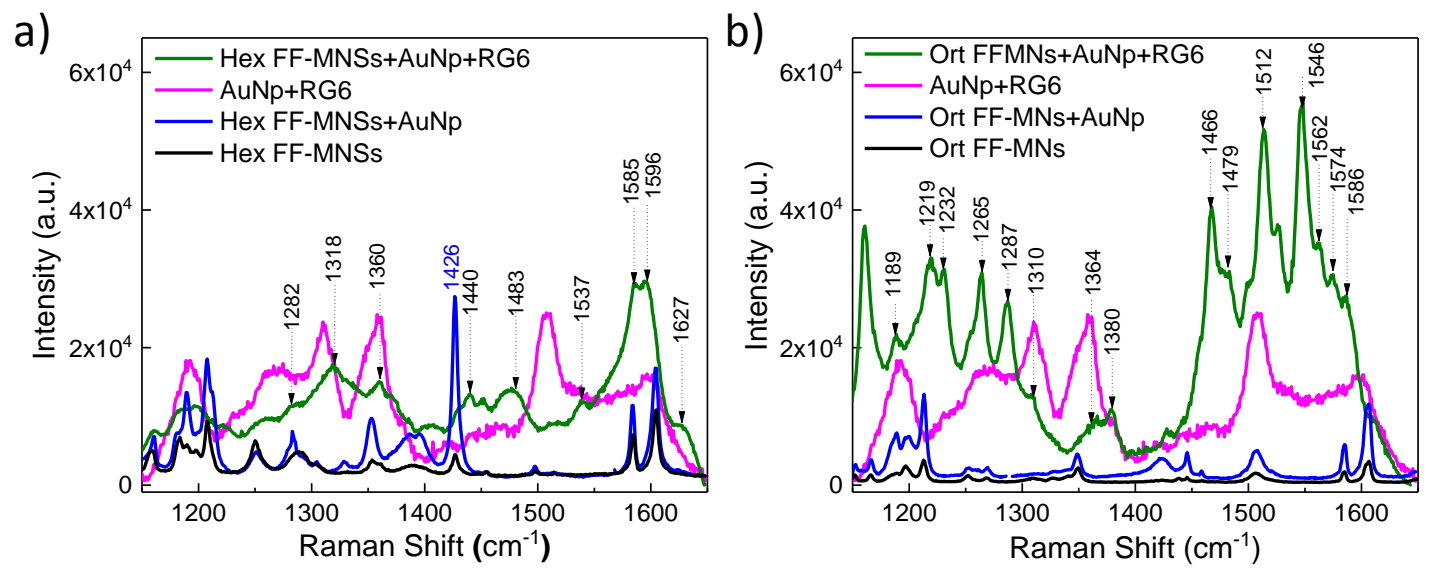

Figure 5.9. Experimental Raman spectra from FF-MNS, FF-MNS+AuNps, FFMNS+AuNps+R6G for the a) hexagonal and b) orthorhombic phase. The Raman spectrum of R6G+AuNps (without any FF-MNS) is shown for comparison in both panels.

\subsubsection{Summarization of the SERS Results}

In summary, we demonstrate that self-assembled FF-MNS films act as templates for the attachment of metal nanoparticles. The materials are prepared in a single step, and without the use of strong acids or corrosive solutions, that can be modified to generate 
more complexes structures in aqueous solution. Both Ag and $\mathrm{Au}$ nanoparticles were used to quantify the SERS activity of the hex and the ort phases of FF-MNS using molecular probes such as RhB or R6G. While both the ort and the hex phase show a strong SERS effect, microscopy images show a more uniform distribution of AgNps in the ort FF-MNS phase without much aggregation. In the hex phase, the metal Nps are seen to deposit and aggregate in channels on top of the FF-MNS hexagonal surface. DFT calculations of the vibrational spectrum from the fundamental unit of FF-MNS with a metal atom and R6G molecule clearly show a higher SERS activity for the ort phase, which may be attributed to a higher chemisorption process in this phase. Using AuNps where both the hex and ort phases of FF-MNS can be accessed with an off-resonance laser excitation, the SERS activity of the ort phase is seen to be higher by a factor of two compared to the hex phase. This work paves the way for biomimetic self-assembled peptide nanostructures as potential SERS substrates for biosensing and biomedical applications

\subsection{Functionalization of FF-MNS with Cobalt Ferrite Nanoparticles}

\subsubsection{Introduction}

Although there are several works that discuss the interaction between protein and selfassembled peptides with metal nanoparticles, there is hardly any work that discusses the role of magnetic nanoparticles with self-assembled peptide nanostructures.

Amongst magnetic nanoparticles, cobalt ferrite $\left(\mathrm{CoFe}_{2} \mathrm{O}_{4}\right.$ or $\left.\mathrm{CFO}\right)$ has received a lot of interest in biomedical applications. ${ }^{81}$ This is predominantly due to its moderate values of saturation magnetization, high magneto-crystalline anisotropy, high Curie temperature, and easy tunability of particle size ${ }^{82}$, facilitating possible use as a contrast agent for magnetic resonance imaging and targeted drug delivery. ${ }^{83-84}$ There are, however, 
limitations of CFO in biomedical applications due to its toxicity and the release and aggregation of cobalt in aqueous solution. These limitations are often mitigated by coating CFO nanoparticles with other nontoxic layers. Moreover, the insulating properties of CFO are attractive in utilizing it as a gate dielectric in organic thin film transistors, setting the stage for magnetic field controlled transistors and devices. ${ }^{85}$

FF-MNS have been evaluated as potential vehicles for drug delivery using a model compound to mimic a drug. ${ }^{86-87}$ The release of the load was modulated, indicating their ability to deliver drugs at constant rates in the body. Cytotoxicity investigations revealed high cell viability up to concentrations of $5 \mathrm{mg} \mathrm{mL}^{-1}$, demonstrating the low toxicity and potential biocompatibility of such nanostructures. ${ }^{87}$ The combination of CFO nanoparticles with FF-MNS provides a less toxic pathway for the application of magnetic nanoparticles in healthcare. Our work here is motivated by a study which showed that $\mathrm{Co}_{3} \mathrm{O}_{4}$ nanoparticles bind to vertically aligned $\mathrm{FF}$ nanostructures, opening up possibilities for energy storage. ${ }^{88}$ However, thus far there is no work that shows the nature of interaction between magnetic nanoparticles and FF, and whether there is any change in the overall magnetic properties.

\subsubsection{Synthesis of FF-MNS:CFO and FET Device Fabrication}

FF-MNS:CFO nanoconjugate was synthesized using liquid phase method described in chapter 1. In this method, $10 \mathrm{mg}$ of FF (purchased from Sigma Aldrich Inc.) and $4 \mathrm{mg}$ of CFO (purchased from Inframat Advanced Materials, Product \# 26F27-ON1, and with an average particle size of $30-40 \mathrm{~nm}$ ) were first dissolved in $100 \mu \mathrm{L}$ of $1,1,1,3,3,3$-hexafluoro2-propanol (HFP) and then the mixtures was diluted using $450 \mu \mathrm{L}$ of DI water. This diluted mixture was immediately ultra-sonicated for about a minute at $10 \mathrm{kHz}$ frequency. After 
that, the mixture was kept in a refrigerator for about 8 hours for aggregation and was centrifuged for about 2 min to separate the solid and liquid phase. Centrifuging helps in purification of the FF-MNS:CFO material. The liquid phase was removed by a pipette from the separated solid-liquid mixture and the solid phase was kept in an oven at $75^{\circ} \mathrm{C}$ until dry.

Bottom-gate top-contact architectures were used for the pentacene FETs and MIS capacitors. First, the dielectric films (FF-MNS or FF-MNS:CFO) were deposited using the solid vapor method, described in Chapter 1. For the FETs, a thin layer (10 nm) of poly (4vinylphenol) was spin-coated prior to the deposition of FFMNS/FF-MNS:CFO. Pentacene was then thermally evaporated $\left(0.3 \AA / \mathrm{s}, 10^{-5}\right.$ mbar $)$ with a thickness of $60 \mathrm{~nm}$ on the dielectric layer. This was followed by thermal evaporation of a 50-nm-thick layer of gold through a shadow mask containing both FET and MIS capacitor structures for the top contacts.

\subsubsection{Magnetism in CFO}

Several minerals found in nature belong to the spinel group. The spinel structure has a cubic unit cell with 56 atoms, 32 oxygen anions distributed in a cubic close packed structure, and 24 cations (of $\mathrm{A}^{2+}$ and $\mathrm{B}^{3+}$ type) occupying 8 of the 64 available tetrahedral $\left(\mathrm{T}_{\mathrm{d}}\right)$ sites and 16 of the 32 available octahedral $\left(\mathrm{O}_{\mathrm{h}}\right)$ sites. In the perfect inverse spinel structure, half of the $\mathrm{B}^{3+}$ occupy the $\mathrm{T}_{\mathrm{d}}$ sites, and $8 \mathrm{~A}^{2+}$ ions and $8 \mathrm{~B}^{3+}$ ions occupy the $\mathrm{O}_{\mathrm{h}}$ sites. CFO belongs to the magnetism-engineered iron oxide $\left(\mathrm{Fe}_{3} \mathrm{O}_{4}\right)$ with ferrimagnetic spin structure (partially inverse spinel) with $\mathrm{O}_{\mathrm{h}}$ and $\mathrm{T}_{\mathrm{d}}$ sites, as represented in Figure 5.10. The $\mathrm{O}_{\mathrm{h}}$ and $\mathrm{T}_{\mathrm{d}}$ sites are represented by brown and green atoms, respectively. $\mathrm{Fe}^{2+}$ and $\mathrm{Fe}^{3+}$ atoms occupying the $\mathrm{O}_{\mathrm{h}}$ sites align parallel to an external magnetic field and $\mathrm{Fe}^{3+}$ atoms in the $\mathrm{T}_{\mathrm{d}}$ 
sites of the FCC- packed oxygen lattice sites are aligned antiparallel to the field. ${ }^{81}$ The total magnetic moment of $\left(\mathrm{Fe}^{3+}\right) \mathrm{T}_{\mathrm{d}}\left(\mathrm{Fe}^{2+} \mathrm{Fe}^{3+}\right) \mathrm{O}_{\mathrm{h}} \mathrm{O}_{4}$ therefore arises from the high spin state of $\mathrm{Fe}^{2+}$ (with $3 \mathrm{~d}^{6}$ electronic configuration) in the $\mathrm{O}_{\mathrm{h}}$ state as the spin of the $\mathrm{Fe}^{3+}\left(\mathrm{O}_{\mathrm{h}}\right)$ and $\mathrm{Fe}^{3+}\left(\mathrm{T}_{\mathrm{d}}\right)$ ions cancel out. The Co ions in CFO predominantly occupy the $\mathrm{O}_{\mathrm{h}}$ site, replacing the $\mathrm{Fe}^{2+}$ ions. The effective Bohr magneton is given by $g[J(J+1)]^{1 / 2}$, where $J$ is the total angular momentum and $g$ is the Landé g-factor. Therefore, the magnetic moment $\left(\mu_{\mathrm{B}}\right)$ per ion for $\mathrm{Fe}^{3+}\left(3 \mathrm{~d}^{5}\right), \mathrm{Fe}^{2+}\left(3 \mathrm{~d}^{6}\right)$, and $\mathrm{Co}^{2+}\left(3 \mathrm{~d}^{7}\right)$ ions are 5.92, 4.90, and 3.87, respectively. Calculation of magnetic moments for different configurations (high spin state) are shown below.
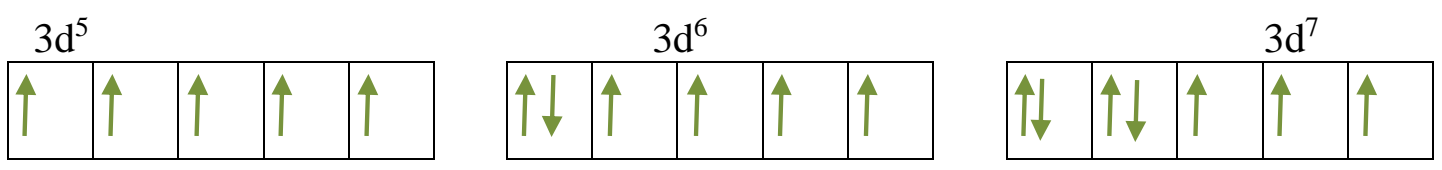

Magnetic moment for $\mathrm{Fe}^{3+}\left(3 \mathrm{~d}^{5}\right)=g[J(J+1)]^{1 / 2}=5.92 \mu_{\mathrm{B}}$ where $g=2, J=5 / 2$.

Magnetic moment for $\mathrm{Fe}^{2+}\left(3 \mathrm{~d}^{6}\right)=g[J(J+1)]^{1 / 2}=4.90 \mu_{\mathrm{B}}$ where $g=2, J=2$.

Magnetic moment for $\mathrm{Co}^{2+}\left(3 \mathrm{~d}^{7}\right)=g[J(J+1)]^{1 / 2}=3.87 \mu_{\mathrm{B}}$ where $g=2, J=3 / 2$.

So the magnetic moment in CFO $\left(\left(\mathrm{Fe}^{3+}\right) \mathrm{T}_{\mathrm{d}}\left(\mathrm{Fe}^{2+} \mathrm{Fe}^{3+}\right) \mathrm{O}_{\mathrm{h}} \mathrm{O}_{4}\right)$ is

$=$ total magnetic moment in tetrahedral site - total magnetic moment in the octahedral site $=5.92-(5.92+3.87)=3.87 \mu_{\mathrm{B}}$.

If $\mathrm{CFO}$ were a perfect inverse spinel structure, where all $\mathrm{Fe}^{2+}$ ions are replaced by $\mathrm{Co}^{2+}$ ions, one would expect the magnetic moment per formula unit to be 3.87 (shown above) since the moments due to $\mathrm{Fe}^{3+}$ at the $\mathrm{T}_{\mathrm{d}}$ and $\mathrm{O}_{\mathrm{h}}$ sites would cancel each other. Typically, in CFO one finds the saturation magnetization to be higher or lower than the ideal value of 3.87, suggesting that Co ions may also occupy the $\mathrm{T}_{\mathrm{d}}$ site and some $\mathrm{Fe}^{2+}$ ions are still 
available at the $\mathrm{O}_{\mathrm{h}}$ site. Our Rietveld analysis of $\mathrm{CFO}$ shows only that about $70 \%$ of $\mathrm{Co}^{2+}$ ions are in the $\mathrm{O}_{\mathrm{h}}$ site, as discussed in Section 5.2.5.

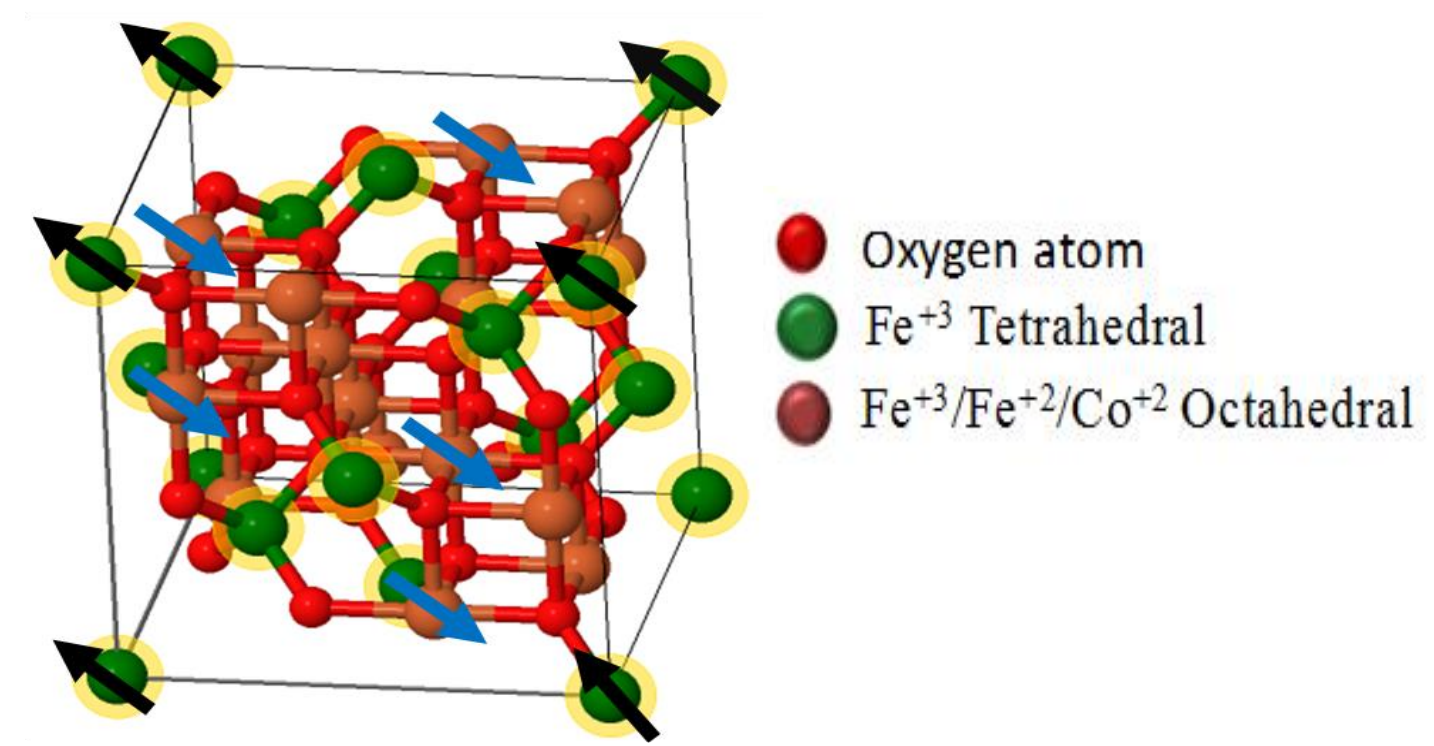

Figure 5.10. The unit cell of $\mathrm{CFO}$ where $\mathrm{Fe}^{3+}$ atoms occupy both the octahedral $\left(\mathrm{O}_{\mathrm{h}}\right)$ and tetrahedral $\left(\mathrm{T}_{\mathrm{d}}\right)$ sites. The octahedral sites are also occupied by $\mathrm{Fe}^{2+}$, which are replaced by $\mathrm{Co}^{2+}$ in CFO. The black and the blue arrows point to the direction of the magnetic moment at the $\mathrm{T}_{\mathrm{d}}$ and $\mathrm{O}_{\mathrm{h}}$ sites, respectively.

\subsubsection{Results}

\subsubsection{XRD and Structure of CFO}

All samples in this work were in the hexagonal phase, which was confirmed by XRD. As the intensity of an XRD pattern depends on the atomic scattering factor ${ }^{89}$ of all elements contained in a sample, among other factors, we first characterized the CFO system by using a Mo source to minimize fluorescence, typically observed when using a $\mathrm{Cu}$ source and studying samples containing elements with $\mathrm{K}$-edge absorptions close to the $\mathrm{Cu}$ target of the tube $($ CoK-edge $=7.709 \mathrm{keV}$; FeK-edge $=7.112 \mathrm{keV}$; CuK-edge $=8.979 \mathrm{keV}) . \mathrm{We}$ characterized the CFO sample as having an inverse spinel structure by using the Rietveld method. ${ }^{90-91}$ The refined unit cell parameters as well as statistical factors (goodness-of-fit 
indicator and $R$-factors ${ }^{92}$ ) were: $a=8.3671(8) \AA, \chi^{2}=1.147, R_{w p}=9.838 \%, R_{\text {Bragg }}=$ $2.137 \%$.

Using a $\mathrm{Cu}$ source we characterized the FF-MNS:CFO system by using the $L, L$ diphenylalanine structure described by Bianchi et al. ${ }^{40}$ and the aforementioned CFO structure. Figure 5.11 (a) and (b) show the Rietveld fitting of the XRD data from CFO and FF-MNS:CFO system respectively. Quantitative phase analysis ${ }^{93}$ using the Rietveld method revealed a phase mixture of $(80.0 \pm 0.4) \mathrm{wt} \%$ of FF-MNS and $(20.0 \pm 0.3) \mathrm{wt} \%$ of CFO. This result is in accordance with the XPS data as well. The refined unit cell parameters and statistical factors are: $a(\mathrm{FF})=24.1251(4) \AA, c(\mathrm{FF})=5.4464(1) \AA ; a(\mathrm{CFO})$ $=8.3684(3) \AA, \chi^{2}=1.416, R_{w p}=2.762 \%, R_{\text {Bragg- } F F}=2.384 \%, R_{\text {Bragg-CFO }}=0.505 \%$. These results clearly show that the FF-MNS is in the hexagonal phase. Although XRD cannot quantify the actual valence of the ions, the structure predicts the presence of $\mathrm{Co}$ and $\mathrm{Fe}$ ions both in the octahedral and tetrahedral sites.
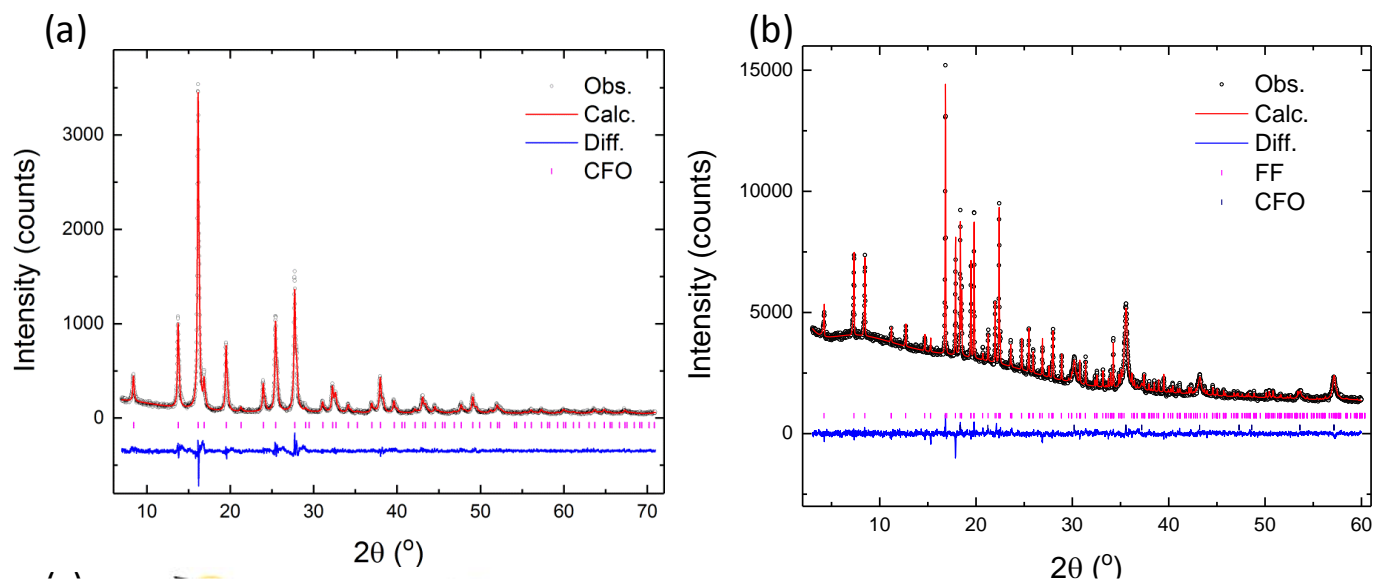

Figure 5.11. (a) and (b) are the Rietveld plots of CFO and FF-MNS:CFO samples respectively. Open black circles represent the observed data; the red line indicates the calculated pattern, the blue line at the bottom displays the difference between observed and calculated data and the vertical magenta bars stand for the Bragg reflections. 


\subsubsection{TEM and SEM}

Figures 5.12 (a) - (d) show the high resolution TEM (HRTEM) images of CFO nanoparticles at various magnifications. These images show high crystallinity of the CFO nanoparticles. Figure 5.12 (e) and (f) are the HRTEM images from a FF nanotube with CFO nanoparticles, and (g) and (h) are the SEM images of a FF nanotube and a FF nanotube with CFO, respectively. Although there is some aggregation, the CFO nanoparticles are seen to be distributed all over the tube. The EDS spectra (which were calibrated using Al, $\mathrm{Cu}$, and $\mathrm{Au}$ ) were also analyzed at several locations of the FF nanotube with $\mathrm{CFO}$, and they confirm the stoichiometry of cobalt ferrite to be $\mathrm{CoFe}_{2} \mathrm{O}_{4}$.

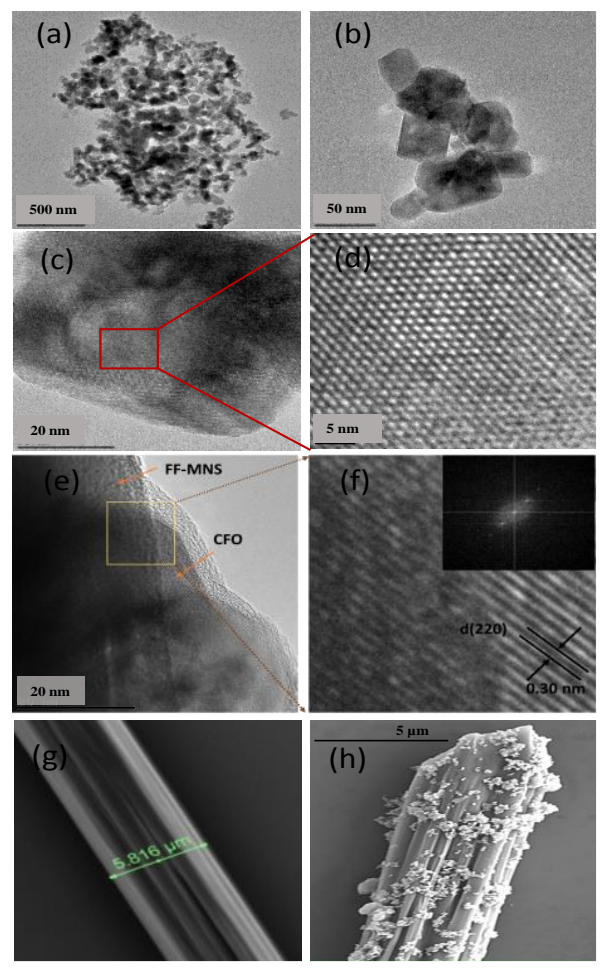

Figure 5.12. (a) - (d) are the TEM images of CFO nanoparticles at various magnifications. (e) High resolution TEM image of FF-MNS:CFO. The yellow box shows the interface between CFO and FF-MNS:CFO, and its magnified image, with lattice fringes, is shown in (f). The inset is the Fourier transform of the image. (g) SEM image of a FF nanotube. (h) SEM image of a FF nanotube with CFO. 
By comparing the HRTEM image (Figure 5.12 (f)) with the XRD data, we obtain the lattice spacing of CFO in FF-MNS:CFO. For the (220) plane in pure CFO, a lattice constant of $a=8.367 \AA$ is obtained from the XRD data, which translates into a $d$ spacing of $0.3 \mathrm{~nm}$. The HRTEM results of FF-MNS:CFO yield an identical $d$ spacing of $0.3 \mathrm{~nm}$ for CFO, suggesting that there are no changes in the lattice constants in CFO after it binds with FFMNS.

\subsubsection{XPS Analysis}

The XPS survey spectra of FF-MNS, CFO, and FF-MNS:CFO are shown in Figure 5.13. The spectra confirm the presence of various elements and their weight fraction in the samples. Quantification of the elements in the survey spectra of FFMNS, FF-MNS:CFO and CFO further confirms that the wt $\%$ of $\mathrm{CFO}$ in FFMNS:CFO is roughly $20-35 \%$, similar to what was found by XRD measurements. High resolution spectra were obtained for $\mathrm{C} 1 \mathrm{~s}, \mathrm{~N} 1 \mathrm{~s}, \mathrm{O} 1 \mathrm{~s}, \mathrm{Fe} 2 \mathrm{p}$, and Co $2 \mathrm{p}$ peaks. The binding energies were estimated by fitting the high resolution peaks in the XPS data with an average error bar of $\pm 0.2 \mathrm{eV}$.

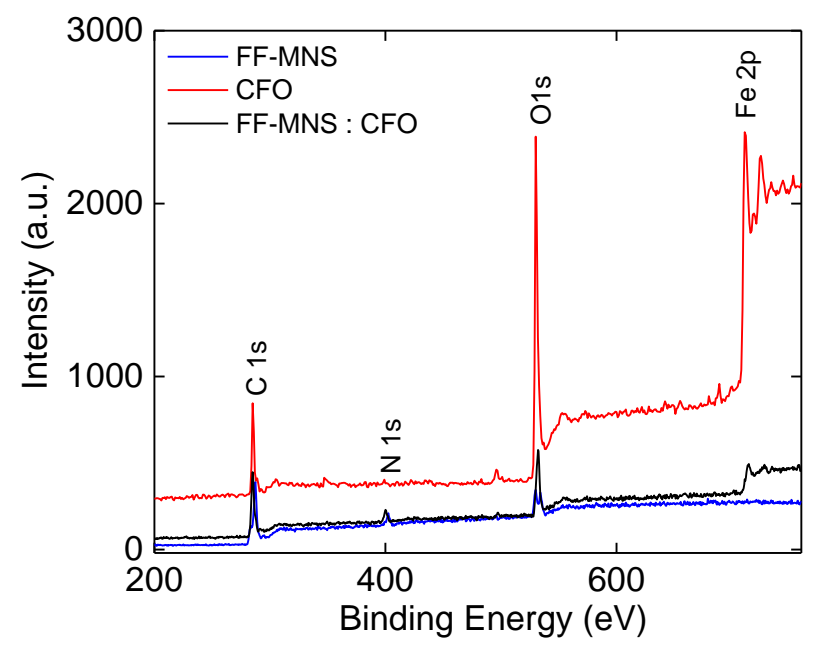

Figure 5.13. XPS spectra of FF-MNS, CFO, and FF-MNS: CFO. 
Figure 5.14 shows the high resolution XPS peaks along with their deconvolution of $\mathrm{C} 1 \mathrm{~s}, \mathrm{~N} 1 \mathrm{~s}$, and $\mathrm{O}$ 1s in FF-MNS and FF-MNS:CFO. The deconvolution of the $\mathrm{C}$ 1s peak in the XPS spectra of FF-MNS and FF-MNS:CFO results in four peaks. The lowest energy peak of $282.4 \mathrm{eV}$ may be attributed to environmental contamination. The energies of 284.8, 286.9, and 290.2 eV in FF-MNS are assigned to C-C, C-N or $\mathrm{C}=\mathrm{O}, \mathrm{O}-\mathrm{C}=\mathrm{O}$ bonds, respectively. Curve fitting of the $\mathrm{C}$ 1s peak in FF-MNS:CFO yields peaks with binding energies of $284.8,286.2$, and $288.6 \mathrm{eV}$. The $\mathrm{N} 1 \mathrm{~s}$ spectrum in FF-MNS was fitted with two peaks centered at $399.9 \mathrm{eV}$ and $402.4 \mathrm{eV}$, attributed to $-\mathrm{N}-\mathrm{C}-$ bond and the $\mathrm{NH} 3$ bond, respectively. These peaks are seen to shift to lower energies in FF-MNS:CFO with corresponding energies of $398.9 \mathrm{eV}$ and 400.9 $\mathrm{eV}$. The deconvolution of the $\mathrm{O} 1 \mathrm{~s} \mathrm{XP}$ spectrum results in four peaks in FF-MNS and three peaks in FF-MNS:CFO. The peak positions in FF-MNS are at 530.3, $531.5,533.2$, and $535.6 \mathrm{eV}$, which correspond to the binding energies of $\mathrm{C}-\mathrm{O}, \mathrm{C}=\mathrm{O}$ or lattice oxygen, hydroxyl group, and chemisorbed oxygen or water, respectively. Although the highest energy peak at $535.6 \mathrm{eV}$ is not observed in FF-MNS:CFO, the binding energies of the other oxygen related peaks do not change. The binding energies obtained by fits to the $\mathrm{C} 1 \mathrm{~s}, \mathrm{~N} 1 \mathrm{~s}$, and $\mathrm{O} 1 \mathrm{~s}$ from FF-MNS and FFMNS:CFO are tabulated in Table 5.4. The largest changes in FF-MNS upon binding with CFO are in the binding energies of the amide bond (including the carbonyl bond) and the amine group, suggesting a charge transfer from CFO.

We further analyzed the Fe 2p peaks in FF-MNS:CFO and CFO to obtain the binding energies related to $\mathrm{Fe}^{3+} / \mathrm{Fe}^{2+}$ ions at the $\mathrm{T}_{\mathrm{d}}$ and $\mathrm{O}_{\mathrm{h}}$ sites of the lattice. The high resolution Fe 2p spectra are shown in Figure 5.14 (d), and the binding energies 
obtained by fits to the Fe 2p XPS data are listed in Table 5.4. The Fe 2p XPS data mainly show two peaks centered at $710 \mathrm{eV}$ and $725 \mathrm{eV}$. The low energy Fe 2p $3 / 2$ peak in CFO is fitted with two peaks centered at $710.2 \mathrm{eV}$ and $712.8 \mathrm{eV}$, which are related to $\mathrm{Fe}^{3+}$ ions at the $\mathrm{O}_{\mathrm{h}}$ site and $\mathrm{T}_{\mathrm{d}}$ site. ${ }^{94-95}$ Some works have attributed one of the $2 \mathrm{p}_{3 / 2}$ to $\mathrm{Fe}^{2+}$ at the $\mathrm{O}_{\mathrm{h}}$ site to the coexistence of two valence states of $\mathrm{Fe} .{ }^{96}$ Our results clearly show an enhancement of the $\mathrm{Fe}^{3+} / \mathrm{Fe}^{2+}$ binding energy in FFMNS:CFO. Both CFO and FF-MNS:CFO samples show a satellite peak at roughly $718 \mathrm{eV}$. The $\mathrm{Fe} 2 \mathrm{p}_{1 / 2}$ peak with a binding energy of $724.3 \mathrm{eV}$ for $\mathrm{Fe}^{3+}\left(\mathrm{O}_{\mathrm{h}}\right.$ site $)$ along with a satellite peak at $732.5 \mathrm{eV}$ appears at the same position in FF-MNS:CFO and CFO.

Chemical shifts in binding energies suggest an electronic redistribution or a charge transfer upon the conjugation of two materials. For the interpretation of shifts in binding energies, the general rule is that the binding energy of the central atom increases as the electronegativity of the attached atoms or groups increases. ${ }^{97} \mathrm{We}$ observe a negative binding energy shift for the $\mathrm{C} 1 \mathrm{~s}$ peak; The 286.9 and the $290.2 \mathrm{eV}$ peaks in FF-MNS are downshifted to 286.2 and $288.6 \mathrm{eV}$ in FF-MNS:CFO. On the other hand, a positive binding energy shift is observed for the $\mathrm{Fe} 2 \mathrm{p}_{3 / 2}$ spectra; the $712.8 \mathrm{eV}$ peak in CFO shifts to 714.5 $\mathrm{eV}$ in FF-MNS:CFO. This is a clear signature of a charge transfer between the Fe ion and $\mathrm{C}=\mathrm{O}$ and $\mathrm{O}-\mathrm{C}=\mathrm{O}$ bonds in $\mathrm{FF}$, keeping in mind that both $\mathrm{O}$ and $\mathrm{N}$ atoms (in the peptide) are more electronegative compared to Fe. Hence, the increase in binding energy of the Fe $2 p_{3 / 2}$ peak suggests that an electron is donated to the peptide. This is only possible from $\mathrm{Fe}^{2+}$, which readily oxidizes to $\mathrm{Fe}^{3+}$. These results further suggest that not all the Co ions occupy the $\mathrm{O}_{\mathrm{h}}$ site. Since $\mathrm{C}$ is more electronegative than Fe according to Pauling's 
electronegativity rule, one would expect the core level of $\mathrm{C}$ to shift towards low binding energy and the core level of Fe to shift towards high binding energy. The chemical shifts in the XPS spectra confirm the nature of the charge transfer between FF-MNS and CFO, where the amine group accepts a negative charge from $\mathrm{Fe}^{2+}$ in $\mathrm{CFO}$, which further affects the carboxyl group. The XPS Co peaks also shift towards higher binding energies in FFMNS:CFO compared to pristine CFO. As the signal to noise ratio is high for the nanocomposite sample, fitting this region becomes difficult although one observes an increase in the binding energy, further suggesting a negative charge transfer from $\mathrm{Co}^{2+}$ to FF-MNS.

The implications of the electrostatic interaction and the charge transfer between CFO and FF-MNS are many fold. The conjugation of bioinspired materials with CFO or other magnetic nanoparticles may change the inherent magnetization properties at the bio-nano interface, opening applications in biomedicine and electronics. Before discussing the magnetization results, we first discuss our Raman scattering and FTIR results from FFMNS and FF-MNS:CFO, further shedding insights into the mechanism of interaction between FF-MNS and CFO. 

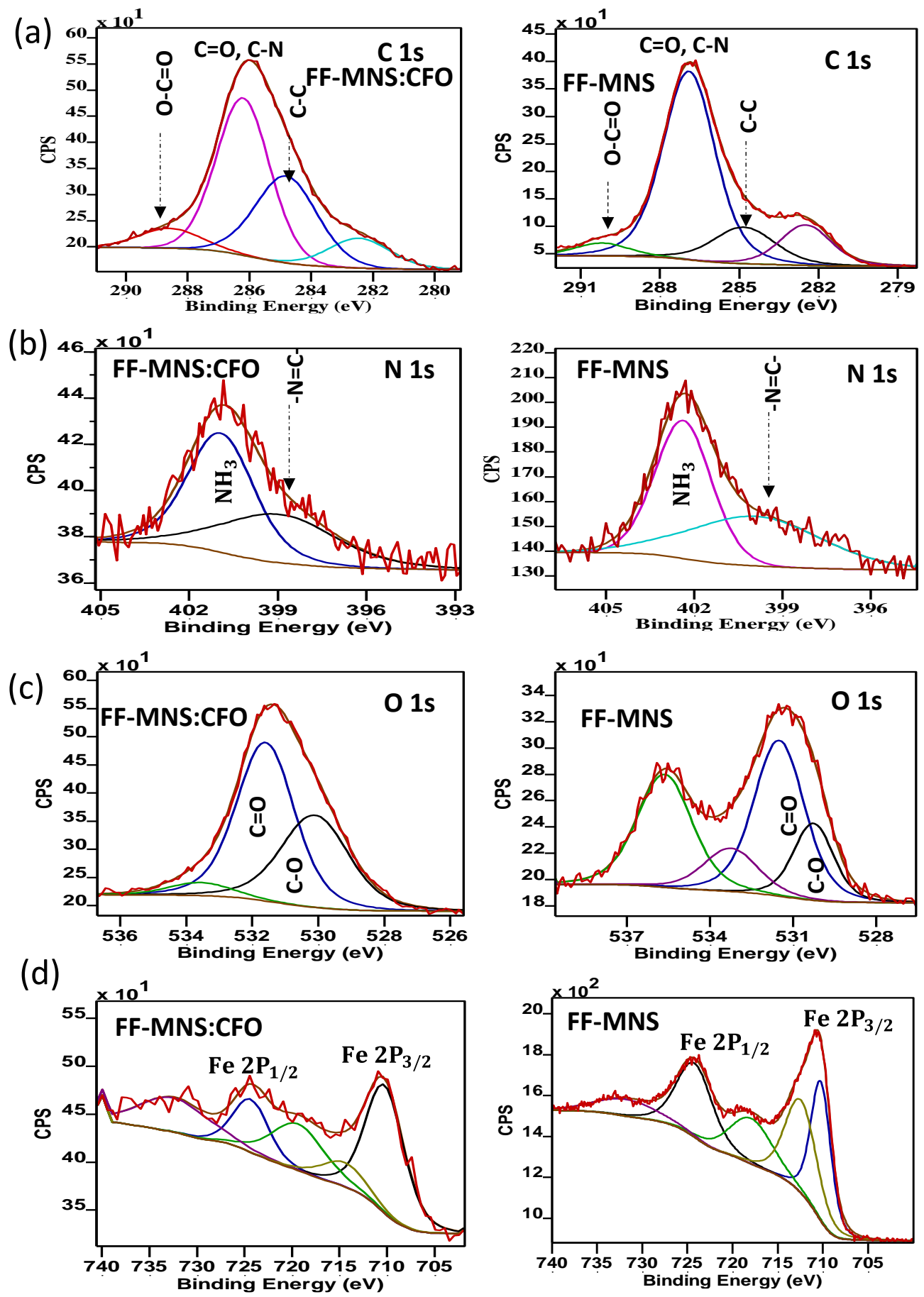

Figure 5.14. High-resolution X-ray photoelectron spectra of (a) $\mathrm{C} 1 \mathrm{~s}$, (b) $\mathrm{N} \mathrm{1s}$, and (c) $\mathrm{O}$ 1s from FF-MNS:CFO ( left panel) and FF-MNS (right panel), and (d) Fe $2 p$ in FFMNS:CFO (left panel) and CFO (right panel). 
Table 5.4. The peak positions and line widths of $\mathrm{C} 1 \mathrm{~s}, \mathrm{~N} 1 \mathrm{~s}, \mathrm{O} 1 \mathrm{~s}$, and $\mathrm{Fe} 2 \mathrm{p}$ by fitting the high resolution XPS data. The numbers in bold signify changes in the binding energy.

\begin{tabular}{|c|c|c|c|c|c|}
\hline \multirow[t]{2}{*}{ Orbital } & \multirow[t]{2}{*}{ Bond } & $\begin{array}{ll}\text { Peak } & \text { Position } \\
(\mathrm{eV}) & \end{array}$ & $\begin{array}{l}\text { FWHM } \\
(\mathrm{eV})\end{array}$ & $\begin{array}{l}\text { Peak Position } \\
(\mathrm{eV})\end{array}$ & $\begin{array}{l}\text { FWHM } \\
(\mathrm{eV})\end{array}$ \\
\hline & & \multicolumn{2}{|l|}{ FF-MNS } & \multicolumn{2}{|l|}{$\begin{array}{l}\text { FF- } \\
\text { MNS:CFO }\end{array}$} \\
\hline \multirow[t]{3}{*}{$\mathrm{C} 1 \mathrm{~s}$} & C-C & 284.8 & 2.26 & 284.8 & 2.34 \\
\hline & $\mathrm{C}-\mathrm{N}, \mathrm{C}=\mathrm{O}$ & 286.9 & 2.33 & 286.2 & 2.03 \\
\hline & $\mathrm{O}-\mathrm{C}=\mathrm{O}$ & 290.2 & 2.41 & 288.6 & 2.34 \\
\hline \multirow[t]{3}{*}{$\mathrm{N} 1 \mathrm{~s}$} & \multirow{2}{*}{$\begin{array}{l}\text { Amide bond -N- } \\
\text { C- }\end{array}$} & 399.9 & 5.24 & 398.9 & 4.54 \\
\hline & & 402.4 & 2.24 & 400.9 & 2.62 \\
\hline & Amine $-\mathrm{NH}_{3}$ & & & & \\
\hline \multirow[t]{5}{*}{ O 1s } & $\mathrm{C}-\mathrm{O}$ & 530.3 & 1.70 & 530.1 & 2.42 \\
\hline & Lattice oxygen & 531.5 & 2.15 & 531.6 & 2.06 \\
\hline & Hydroxyl & 533.3 & 2.32 & 533.6 & 2.24 \\
\hline & Chemisorbed & 535.6 & 2.33 & & \\
\hline & & \multicolumn{2}{|l|}{ CFO } & \multicolumn{2}{|l|}{$\begin{array}{l}\text { FF- } \\
\text { MNS:CFO }\end{array}$} \\
\hline \multirow[t]{3}{*}{$\mathrm{Fe} 2 \mathrm{p}_{3 / 2}$} & $\mathrm{Fe}^{3+}\left(\mathrm{O}_{\mathrm{h}}\right)$ & 710.2 & 2.61 & 710.2 & 4.79 \\
\hline & $\mathrm{Fe}^{3+} / \mathrm{Fe}^{2+}$ & 712.8 & 4.07 & 714.5 & 5.44 \\
\hline & satellite peak & 717.9 & 5.54 & 719.4 & 6.52 \\
\hline \multirow[t]{2}{*}{$\mathrm{Fe} 2 \mathrm{p}_{1 / 2}$} & $\mathrm{Fe}^{3+}\left(\mathrm{O}_{\mathrm{h}}\right)$ & 724.3 & 4.68 & 724.3 & 4.30 \\
\hline & satellite peak & 731.8 & 7.95 & 732.5 & 8.66 \\
\hline
\end{tabular}




\subsubsection{Raman Scattering and FTIR}

The spinel structure of ferrites show 39 vibrational modes out of which six phonon modes are Raman active $\left(2 \mathrm{~A}_{1 \mathrm{~g}}, \mathrm{E}_{\mathrm{g}}\right.$, and $\left.3 \mathrm{~T}_{2 \mathrm{~g}}\right)$. The $\mathrm{A}_{1 \mathrm{~g}}$ mode, at $680 \mathrm{~cm}^{-1}$, in CFO corresponds to a symmetric stretch of the $\mathrm{O}$ atoms along the $\mathrm{Fe} / \mathrm{Co}-\mathrm{O}$ bond. From a detailed work on ferrites and chromites, the $\mathrm{A}_{\lg }$ mode seems to depend more on the $\mathrm{O}_{\mathrm{h}}$ ions than $\mathrm{T}_{\mathrm{d}}$ ions. ${ }^{98}$ The Raman spectrum of FF-MNS:CFO shows characteristic features both from FFMNS and CFO. Comparing the Raman peak positions of the nanocomposite sample to pristine FF-MNS and CFO allow an understanding of how the chemical bonds are affected.

To highlight the changes, we show three regions in Figure 5.15: the $400-700 \mathrm{~cm}^{-1}$ and $1300-1500 \mathrm{~cm}^{-1}$ range. Figure 5.15 (a) and (b) compare key features in the Raman spectrum of CFO and FF-MNS:CFO in the $400-700 \mathrm{~cm}^{-1}$ range. The low energy $\mathrm{T}_{2 \mathrm{~g}}$ Raman peak in CFO is clearly seen in the pristine sample at $288 \mathrm{~cm}^{-1}$. The presence of FF-MNS Raman peaks in the nanocomposite sample makes it difficult to obtain the peak positions originating only from $\mathrm{CFO}$ in the low energy range. The strongest Raman peak in $\mathrm{CFO}$ near $680 \mathrm{~cm}^{-1}$, which is the $A_{1 g}$ mode, is free from any FF-MNS Raman peaks in that region. This $\mathrm{A}_{1 \mathrm{~g}}$ mode observed at $675 \mathrm{~cm}^{-1}$ in CFO hardens and shifts to $685 \mathrm{~cm}^{-1}$ in FFMNS:CFO. The shift to higher frequencies (for the Stokes spectrum) in FF-MNS:CFO compared to CFO can be clearly seen upon overlaying the two spectra. The $619 \mathrm{~cm}^{-1}$ Raman peak in FF-MNS:CFO originates from the hexagonal phase of FF-MNS itself. The $458 \mathrm{~cm}^{-1}$ Raman peak of CFO also shifts to higher frequencies in FF-MNS:CFO.

The change in the $\mathrm{A}_{1 \mathrm{~g}}$ mode frequency position in $\mathrm{CFO}$ compared with the nanocomposite sample is an indication of a charge transfer process. The amine group accepts an electron from $\mathrm{Fe}^{2+}$ ions (most likely at the $\mathrm{O}_{\mathrm{h}}$ site) or $\mathrm{Co}^{2+}$ ions, affecting the 
frequency of the adjacent carboxyl group. The hardening of the Raman peak position may be further understood based on our XPS results where the binding energy of the Fe 2p $3 / 2$ increases. Since ionic bonds may have some covalent nature, a higher binding energy suggests an enhancement of the stiffness constant for the $\mathrm{Fe}^{2+}-\mathrm{O}$ bonds, resulting in the hardening of the $\mathrm{CFO} \mathrm{A}_{\lg }$ mode in the nanocomposite sample.

(a)
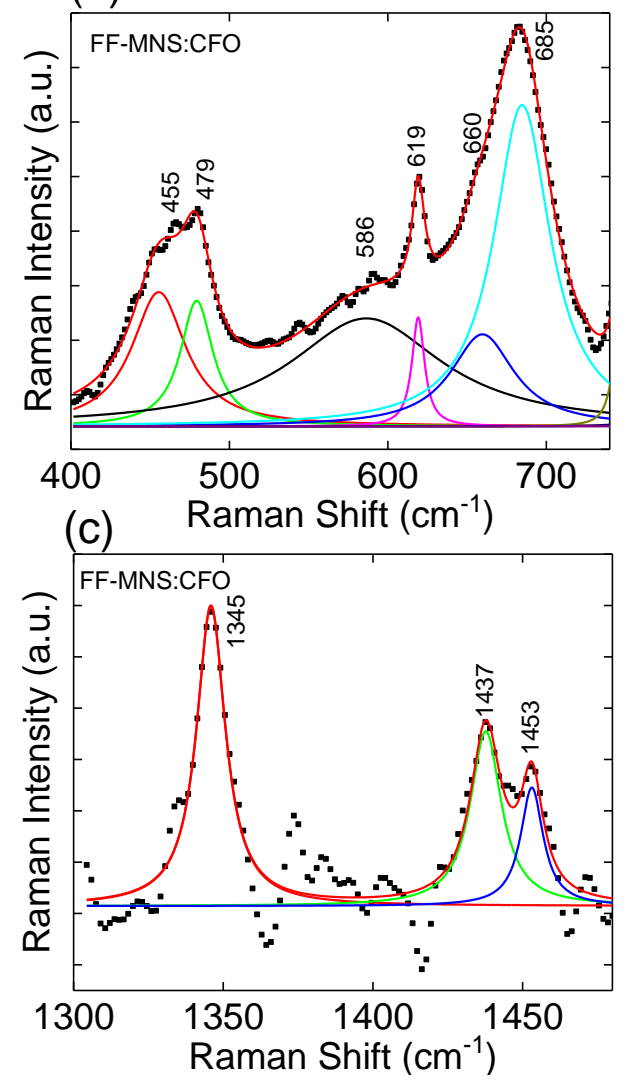

(b)

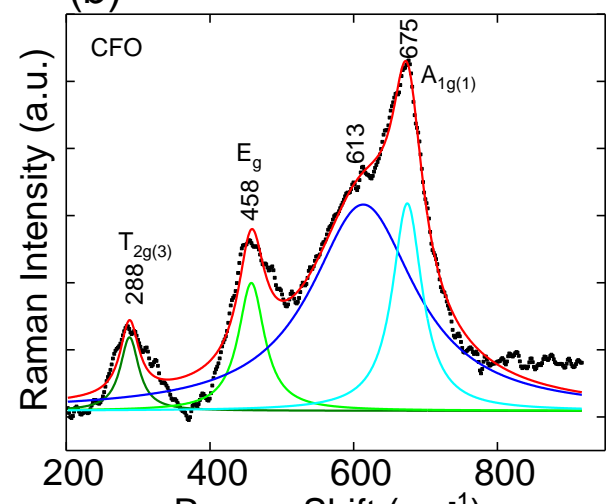

(d) Raman Shift $\left(\mathrm{cm}^{-1}\right)$

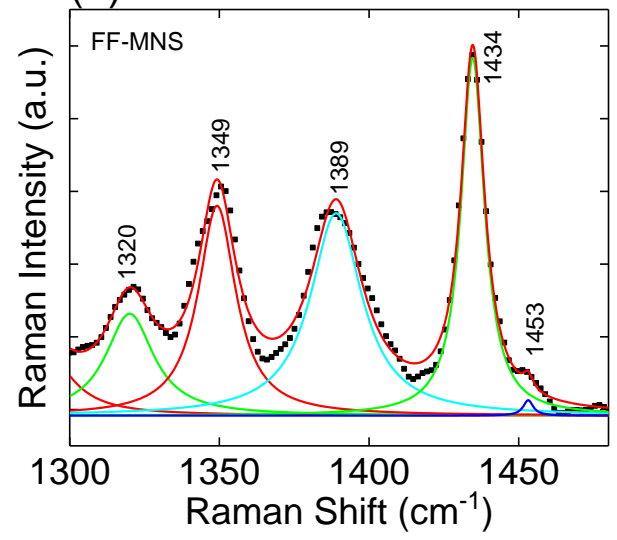

Figure 5.15. (a) and (b) Raman spectra of the predominant CFO vibrational modes in FFMNS:CFO and CFO, respectively. (c) and (d) the $1300 \mathrm{~cm}-1$ region in FF-MNS:CFO and CFO, respectively.

One also observes changes in the FF-MNS Raman frequencies; the $1349 \mathrm{~cm}^{-1}$ Raman peak in FF-MNS, ${ }^{99}$ which arises from the vibration mode of amide III bond, softens to $1345 \mathrm{~cm}^{-1}$ in FF-MNS:CFO. In particular, the intensity ratio of the $1434 \mathrm{~cm}^{-1}$ to the 1349 $\mathrm{cm}^{-1}$ Raman peaks in FF-MNS decreases in the nanocomposite sample, most likely 
reflecting a change in the torsional angle of the peptide bond. The $1430 \mathrm{~cm}^{-1}$ peak in FFMNS originates from a bending motion of the peptide bond and the amine group, whereas the $1349 \mathrm{~cm}^{-1}$ peak arises from a bending of the amine group. Some of these vibration modes has been discussed in Chapter 3 and Chapter 4.

The FTIR data complements the Raman scattering data and yields information on the vibrations originating from the carboxyl and amine groups upon charge transfer. Figure 5.16 (a) and (b) show the FTIR spectra of FF-MNS and FF-MNS:CFO in the range from $500 \mathrm{~cm}^{-1}$ to $4000 \mathrm{~cm}^{-1}$. The frequencies of the amide bonds are shown in Figure 5.16 (c) and (d). We note that all the absorption peaks in the FTIR spectra are from FF-MNS. The strong vibrations corresponding to the amide group are seen to downshift in energy in FFMNS:CFO; The $1672 \mathrm{~cm}^{-1}$ peak in FF-MNS, which arises from the vibration of amide I (stretching vibration of $\mathrm{C}=\mathrm{O}$ ), shifts to $1668 \mathrm{~cm}^{-1}$, and the $3276 \mathrm{~cm}^{-1}$ peak in FF-MNS from the amide A vibration (NH stretching) shifts to $3266 \mathrm{~cm}^{-1}$ in FF-MNS:CFO. The amide $\mathrm{V}$ (out of plane $\mathrm{NH}$ bending) mode at $833 \mathrm{~cm}^{-1}$ in FF-MNS shifts to $815 \mathrm{~cm}^{-1}$ in FFMNS:CFO. The downshift of these frequencies in the nanocomposite sample may be linked to the XPS data where the binding energy of the $\mathrm{C}=\mathrm{O}$ and $\mathrm{NH}_{3}$ bonds decreases, reflecting a decrease in the stiffness constant of the bonds. The trends are less clear for the FTIR peaks that originate from coupled vibrations. One example is the $1546 \mathrm{~cm}^{-1}$ peak in $\mathrm{FF}$ MNS, which arises from the amide II bond vibration (associated with coupled C-N stretching and N-H bending vibration), shifting to a higher frequency of $1550 \mathrm{~cm}^{-1}$ in FFMNS:CFO.

Overall, the Raman data highlight changes in CFO frequencies upon binding with FFMNS and the FTIR data show changes in the vibrational frequencies associated with the 
amine and carboxyl groups of FF-MNS. Although the charge transfers from $\mathrm{Fe}^{2+}$ and $\mathrm{Co}^{2+}$ ions to the amine group, since the carboxyl group is in close proximity to the amine group, the frequency related to the $\mathrm{C}=\mathrm{O}$ stretching motion is also affected.
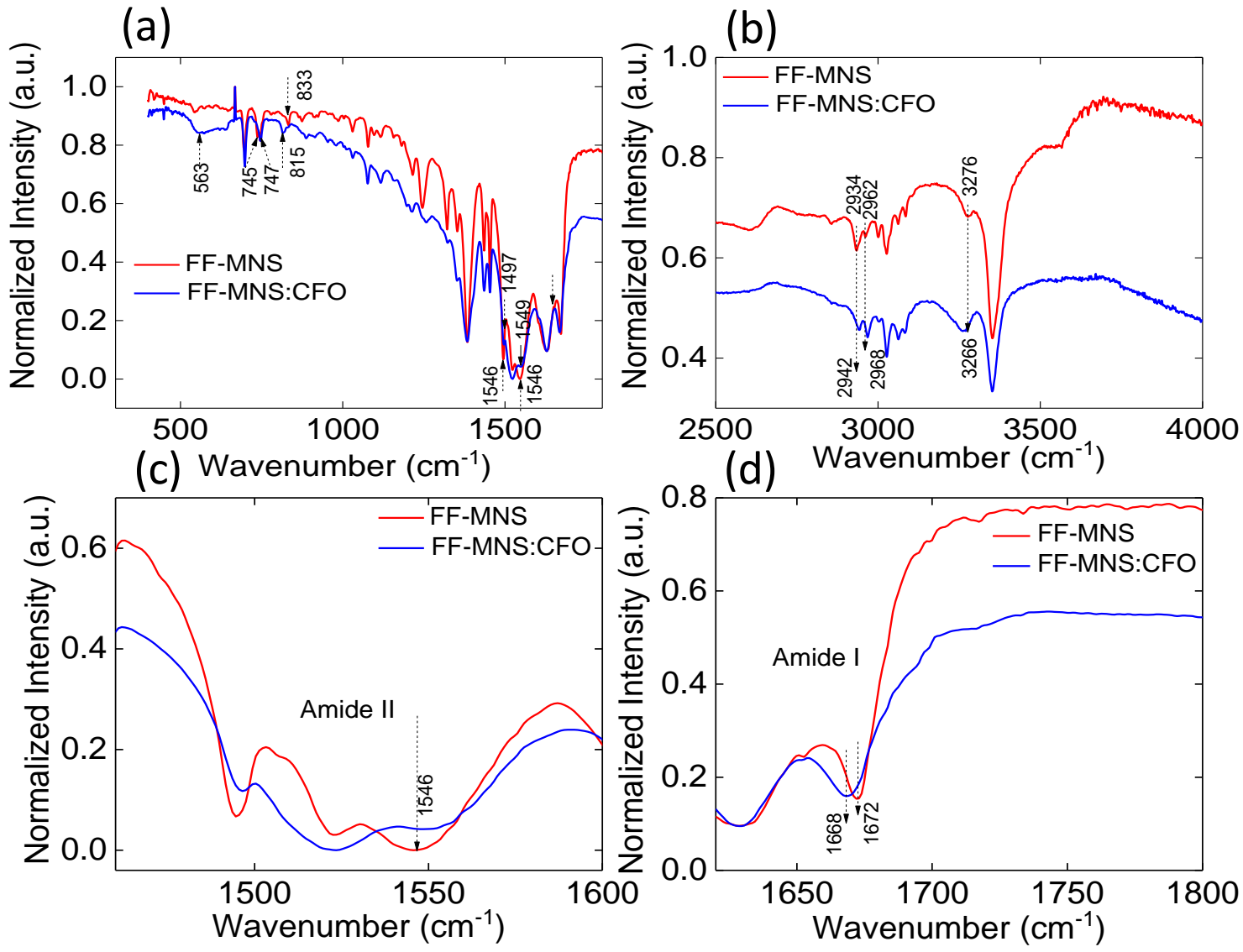

Figure 5.16. FTIR spectra from FF-MNS and FF-MNS:CFO. The spectra in the range: 500 $\mathrm{cm}^{-1}$ to $4000 \mathrm{~cm}^{-1}$ are shown in (a) and (b). The high frequency region is shown in (b). The region of the amide II and I bonds are shown in (c) and (d), respectively.

\subsubsection{Magnetization}

The magnetization data (as a function of the external field $(\mathrm{H})$ ) for the powder samples at $300 \mathrm{~K}$ are shown in Figure 5.17. The full magnetization data till $50 \mathrm{kOe}$ is shown in Figure 5.18. Due to the lack of domains in nanoparticles, the data represents a typical magnetization curve for superparamagnetism with a very low coercive field. Similar magnetization curves (with almost no hysteresis) have also been observed for cobalt iron 
carbide nanoparticles. ${ }^{100}$ The saturation magnetization $\left(\mathrm{M}_{\mathrm{s}}\right)$ for pure $\mathrm{CFO}$ at $5000 \mathrm{Oe}$ is measured as $3.6 \mu_{\text {B }}$ per formula unit (f.u.). Experimentally, we obtain a lower magnetic moment/f.u. compared to the maximum theoretical value of $\mathrm{CFO}$ as the measurements were performed at room temperature. We note that FF-MNS:CFO has 20-35\% of CFO, confirmed by XRD and XPS. For the magnetization measurements, we assume $35 \mathrm{wt} \%$ of $\mathrm{CFO}$ in the nanocomposite sample. As shown in Figure 5.17, $\mathrm{M}_{\mathrm{s}}$ for FF-MNS:CFO is 4.6 $\mu_{\mathrm{B}} / \mathrm{f} . \mathrm{u}$.; compared to CFO, it is approximately $28 \%$ increase in $\mathrm{M}_{\mathrm{s}}$. Using the high field value (Figure 5.18), the enhancement in $\mathrm{M}_{\mathrm{s}}$ is approximately $20 \%$ for FF-MNS:CFO. The enhanced value of $\mathrm{M}_{\mathrm{s}}$ is an indication that upon binding with FF-MNS, the overall ionic distribution and valency of CFO changes.

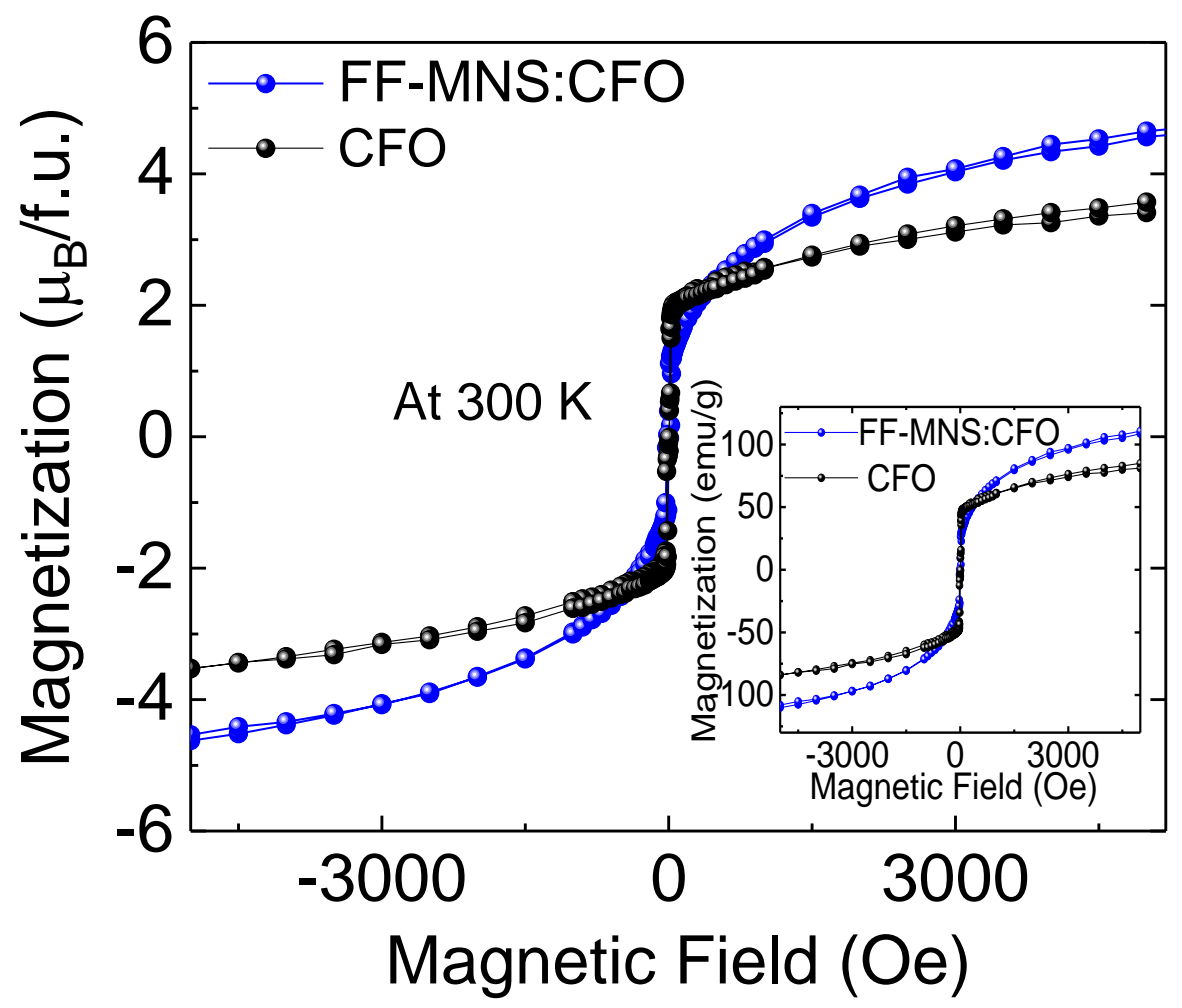

Figure 5.17. Magnetic hysteresis loops for CFO (black) and FF-MNS:CFO (blue) at room temperature. The inset shows the same data with the unit of magnetization in emu/g. 
The magnetization $(M)$ data for both CFO and FF-MNS:CFO follows the modified Langevin function rather than the Langevin function, which is given by ${ }^{101}$

$$
M=M_{o} \mathcal{L}\left(\frac{\mu_{p} H}{k_{B} T}\right)+\chi_{a} H
$$

where $\mu_{p}$ is the average magnetic moment per particle, $\mathcal{L}(x) \equiv \operatorname{coth} x-\frac{1}{x}$ is the Langevin function, $M_{0}$ is the saturation magnetization, and $\chi_{a}$ is the susceptibility of randomly oriented magnetic particles or any other source of deviation from an ideal magnetic order due to disorder. This behavior has been observed in antiferromagnetic nanoparticles such as ferritin ${ }^{102}$ and $\mathrm{NiO}^{103}$ as well as for magnetic nanocrystals. ${ }^{104}$ The need for a modified Langevin function was observed as early as in the late 1930s for colloidal magnetite particles. ${ }^{105}$ The fitted data to the modified Langevin function are shown in Figure 5.18, and details of the fit are given in Table 5.5.

Table 5.5. Fits from the modified Langevin-type function for CFO and FF-MNS:CFO.

\begin{tabular}{cccc}
\hline Samples & $M_{o}(\mathrm{emu} / \mathrm{g})$ & $\mu_{p}\left(\mu_{B}\right)$ & $\chi_{a}(\mathrm{emu} / \mathrm{g} \mathrm{Oe})$ \\
\hline CFO & $103.3 \pm 0.3$ & $4306.7 \pm 43.5$ & $1.33 \times 10^{-5} \pm$ \\
& & & $7.0 \times 10^{-6}$ \\
FF- & $122.9 \pm 5.1$ & $8000 \pm 691$ & $6.17 \times 10^{-4} \pm$ \\
MNS:CFO & & & $1.9 \times 10^{-5}$ \\
\hline
\end{tabular}

The modified Langevin function fits the CFO data very well Figure 5.18. Using the above model (equation (5.1)), the saturation magnetization values, $M_{0}$, of FF-MNS:CFO is higher than that of CFO. The additional $\chi_{a} H$ term in the modified Langevin function also contributes to the magnetization. As seen in Table 5.5, the value for $\chi_{a}$ is also higher in FF-MNS:CFO compared to pure CFO. $\mu_{p}$, which denotes the average moment per particle, is also higher in FF-MNS:CFO. This could suggest slightly higher particle size due to aggregation. The values obtained here may be compared with other nanoparticles where 
the modified Langevin function was used; the reported values for $M_{0}, \mu_{p}$, and $\chi_{a}$ in $\mathrm{NiO}$ nanoparticles are: $1.30 \mathrm{emu} / \mathrm{gm}, 1967 \mu_{B}$, and $34 \times 10^{-6} \mathrm{emu} / \mathrm{g}$ Oe respectively ${ }^{42}$
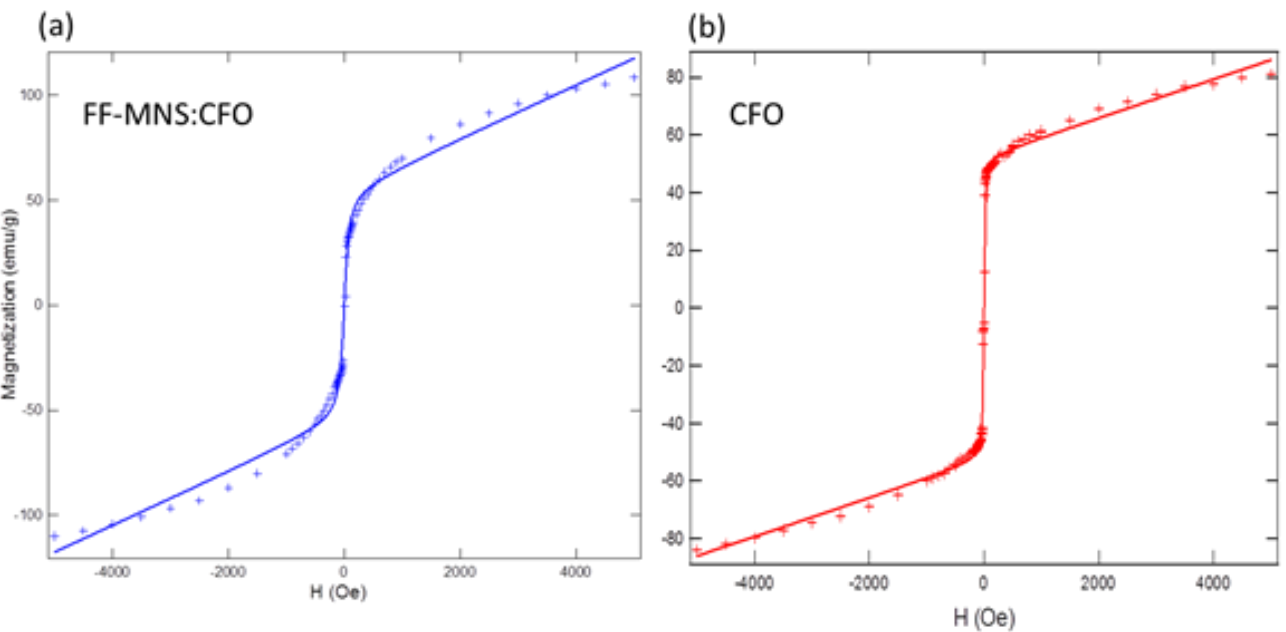

Figure 5.18. Magnetization data for $\mathrm{CFO}$ and FF-MNS:CFO (high magnetic fields) at room temperature along with the fitted modified Langevin function as discussed below. The solid lines in (a) and (b) are the fits.

Ferromagnetic to antiferromagnetic transition, the coercive field, remnant magnetization, $\mathbf{M}_{\mathrm{s}}$, exchange fields, and the Curie temperatures are known to change in doped CFO compared to pristine CFO. ${ }^{106-108}$ Increase in $\mathrm{M}_{\mathrm{s}}$ is also seen in CFO/graphene oxide (GFO) nanofibers with increasing GFO content. ${ }^{109}$ In the next section we discuss a few models that explain the behavior of enhanced magnetization in the nanocomposite sample.

\subsubsection{Discussion of Enhanced Magnetic Properties in FF-MNS:CFO}

XPS, optical spectroscopy, and magnetization results all point in the direction of a charge transfer mechanism between $\mathrm{CFO}$ and FF-MNS. A recent study on the interaction between amino acids and magnetite elucidates its nature, which is governed by the polarity of the side chains and is mediated primarily by the carboxyl groups. ${ }^{110}$ Other examples include bonding of CFO particles to p-type carbon nanotubes, which is facilitated through 
the metal-oxygen bridges, resulting in efficient ion diffusion. ${ }^{111}$ In another work on polyaniline (PANI) and CFO, the pyrrolic-N sites play a role. ${ }^{112}$

XPS and Raman scattering both show that Fe/Co-O bonds stiffen in the nanocomposite sample. Further, the magnetization data clearly show an increase in Ms when CFO binds with FF-MNS. Thus, the nature of the charge transfer and the cation redistribution in the nanocomposite is such that there is an overall increase in the magnetic moment. We first show that a few scenarios can be easily ruled out. Since the binding energies related to both $\mathrm{Fe}$ and Co increase, and as they are less electronegative than $\mathrm{C}, \mathrm{N}$, and $\mathrm{O}$, electrons will be donated from CFO to FF-MNS. Both Fe and Co appear as mixed valence in the CFO crystal structure, and since neither $\mathrm{Fe}^{4+}$ nor $\mathrm{Co}^{4+}$ can exist in $\mathrm{CFO}$, the only possibilities for a charge transfer would be from $\mathrm{Fe}^{2+}$ and $\mathrm{Co}^{2+}$, such that they are oxidized to $\mathrm{Fe}^{3+}$ and $\mathrm{Co}^{3+}$. The next question that arises is which sites, $\mathrm{T}_{\mathrm{d}}$ or $\mathrm{O}_{\mathrm{h}}$, are involved? To answer this question, we need to consider both the increase in Ms and changes observed in XPS when CFO binds to FF-MNS.

XRD data show that Co may be occupied both in the $T_{d}$ as well as in the $\mathrm{O}_{\mathrm{h}}$ sites, ${ }^{113}$ which is also seen from our Rietveld analysis. Assuming a perfect inverse spinel structure for $\mathrm{CFO}$, our results imply that not all the $\mathrm{Fe}^{2+}$ ions in the $\mathrm{O}_{\mathrm{h}}$ site are replaced by $\mathrm{Co}^{2+}$. Our Rietveld analysis shows that $\sim 70 \%$ of Co ions are in the $\mathrm{O}_{\mathrm{h}}$ site. Unfortunately, solely from single wavelength $x$-ray analysis, it is difficult to obtain the actual valence states of the ions. Resonance XRD methods using a synchrotron source have been effective for obtaining the mixed valence ratios of $\mathrm{Fe}^{2+} / \mathrm{Fe}^{3+}{ }^{89}$ We estimate the net magnetic moment per f.u. for different configurations (and spin states) of Fe and Co ions in $\mathrm{T}_{\mathrm{d}}$ or $\mathrm{O}_{\mathrm{h}}$ positions, shown in Table 5.7. The crystalline electric field splitting in CFO due to the $\mathrm{O}_{\mathrm{h}}$ symmetry 
is not prominent so a high spin state is favorable for the $\mathrm{d}$ electrons, as also supported by recent experimental findings in $\mathrm{Fe}_{3} \mathrm{O}_{4}$ as well as in $\mathrm{CFO} .{ }^{114-115}$

Shown in Table 5.7 are two possible situations (one set shown in bold and the other in italics) for enhanced magnetization in FF-MNS:CFO compared to pure CFO. We consider the first model where $\mathrm{Fe}^{2+}$ in the $\mathrm{Oh}$ site has all been replaced by $\mathrm{Co}^{2+}$, resulting in the configuration of $\left[\mathrm{Fe}^{3+}\right] \mathrm{T}_{\mathrm{d}}\left[\mathrm{Co}^{2+}, \mathrm{Fe}^{3+}\right] \mathrm{O}_{\mathrm{h}} \mathrm{O}_{4}$, with a magnetic moment of $3.87 \mu_{\mathrm{B}}$ for CFO. CFO in such a configuration when bound to FF-MNS (in FF-MNS:CFO) is expected to give up an electron resulting in $\left[\mathrm{Fe}^{3+}\right] \mathrm{T}_{\mathrm{d}}\left[\mathrm{Co}^{3+} \mathrm{Fe}^{3+}\right] \mathrm{O}_{\mathrm{h}} \mathrm{O}_{4}$ with a magnetic moment of 4.90

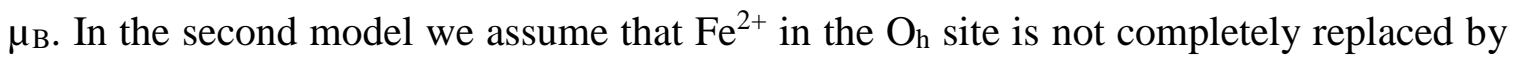
$\mathrm{Co}^{2+}$. With $70 \%$ of Co ions in the $\mathrm{O}_{\mathrm{h}}$ site (which is close to the Rietveld analysis) and 30\% in the $T_{d}$ site and 1:2 fraction for Co:Fe (as seen in EDS), we assume a perfect inverse spinel structure with 8 ions in the $T_{d}$ site and 16 ions in the $\mathrm{O}_{\mathrm{h}}$ site. These assumptions result in a CFO configuration of $\left[\mathrm{Fe}^{3+}(0.7) \mathrm{Co}^{3+}(0.3)\right] \mathrm{T}_{\mathrm{d}}\left[\mathrm{Fe}^{2+}(0.3) \mathrm{Co}^{2+}(0.7) \mathrm{Fe}^{3+}\right] \mathrm{O}_{\mathrm{h}} \mathrm{O}_{4}$, where the numbers in the bracket denote their fraction, yielding a net magnetic moment of $4.49 \mu_{\mathrm{B}}$. CFO in such a configuration bound to FF-MNS will change to $\left[\mathrm{Fe}^{3+}(0.7)\right.$ $\left.\mathrm{Co}^{3+}(0.3)\right] \mathrm{T}_{\mathrm{d}}\left[\mathrm{Fe}^{3+}(0.3) \mathrm{Co}^{3+}(0.7) \mathrm{Fe}^{3+}\right] \mathrm{O}_{\mathrm{h}} \mathrm{O}_{4}$ with a net magnetic moment of $5.52 \mu_{\mathrm{B}}$. Both models show an enhancement in magnetization by $21-23 \%$ for FF-MNS:CFO, similar to our experimental observation.

Table 5.6. Magnetic moment of atoms for different configuration.

\begin{tabular}{ccc}
\hline ConFigure & High Spin & Low Spin \\
\hline $\mathrm{d}^{5}$ & 5.92 & 1.73 \\
$\mathrm{~d}^{6}$ & 4.90 & 0 \\
$\mathrm{~d}^{7}$ & 3.87 & 1.73
\end{tabular}

Table 5.7. Net magnetic moment for different configuration of $\mathrm{Fe}_{3} \mathrm{O}_{4}, \mathrm{CoFe}_{2} \mathrm{O} 4$, and FFMNS:CFO. 


\begin{tabular}{|c|c|c|c|c|c|}
\hline Material & $\begin{array}{l}\text { Ions in } \\
\text { Tetrahedral site } \\
\text { Magnetic } \\
\text { Moment }\left(\mu_{\mathrm{B}}\right)\end{array}$ & $\begin{array}{l}\text { Ions in Octahedral site } \\
\text { Magnetic Moment }\left(\mu_{\mathrm{B}}\right)\end{array}$ & & $\begin{array}{l}\text { Spin } \\
\text { State }\end{array}$ & $\begin{array}{l}\text { Magnetic } \\
\text { Moment } \\
\text { per f.u. } \\
\left(\mu_{\mathrm{B}}\right)\end{array}$ \\
\hline \multirow[t]{2}{*}{$\mathrm{Fe}_{3} \mathrm{O}_{4}$} & $\begin{array}{l}\mathrm{Fe}^{+3}\left(\mathrm{~d}^{5}\right) \\
5.92\end{array}$ & $\begin{array}{l}\mathrm{Fe}^{2+}\left(\mathrm{d}^{6}\right) \\
4.90\end{array}$ & $\begin{array}{l}\mathrm{Fe}^{3+}\left(\mathrm{d}^{5}\right) \\
5.92\end{array}$ & High & 4.90 \\
\hline & 5.92 & 0 & 1.73 & Low & 4.19 \\
\hline \multirow{2}{*}{$\mathrm{CoFe}_{2} \mathrm{O} 4$} & $\begin{array}{l}\mathrm{Fe}^{+3}\left(\mathbf{d}^{5}\right) \\
5.92\end{array}$ & $\begin{array}{l}\mathrm{Co}^{2+}\left(d^{7}\right) \\
3.87\end{array}$ & $\begin{array}{l}\mathrm{Fe}^{3+}\left(\mathbf{d}^{5}\right) \\
5.92\end{array}$ & High & 3.87 \\
\hline & 5.92 & 1.73 & 1.73 & Low & 2.46 \\
\hline \multirow[t]{2}{*}{$\mathrm{CoFe}_{2} \mathrm{O} 4$} & $\begin{array}{l}\left(F e_{0.7}\left(d^{5}\right)\right. \\
\left.C o_{0.3}\left(d^{6}\right)\right)^{+3} \\
5.61\end{array}$ & $\begin{array}{l}\left.\left(C_{0.7}\left(d^{7}\right) F e_{0.3\left(d^{6}\right.}\right)\right)^{2+} \\
4.18\end{array}$ & $\begin{array}{l}F e^{+3}\left(d^{5}\right) \\
5.92\end{array}$ & High & 4.49 \\
\hline & 5.61 & 1.21 & 1.73 & Low & 2.67 \\
\hline \multirow{2}{*}{$\begin{array}{l}\text { FF- } \\
\text { MNS:CFO }\end{array}$} & $\begin{array}{l}(\mathbf{F e})^{3+}\left(d^{5}\right) \\
5.92\end{array}$ & $\begin{array}{l}\mathrm{Co}^{3+}\left(\mathrm{d}^{6}\right) \\
4.90\end{array}$ & $\begin{array}{l}\mathrm{Fe}^{+3}\left(\mathrm{~d}^{5}\right) \\
5.92\end{array}$ & High & 4.90 \\
\hline & 5.92 & 0 & 1.73 & Low & 4.19 \\
\hline \multirow[t]{2}{*}{$\begin{array}{l}F F- \\
M N S: C F O\end{array}$} & $\begin{array}{l}F e_{0.7}\left(d^{5}\right), \\
\left.C o_{0.3}\left(d^{6}\right)\right)^{+3} \\
5.61\end{array}$ & $\begin{array}{l}\left.\left(\operatorname{Co}_{0.7}\left(d^{6}\right) F e_{0.3\left(d^{5}\right.}\right)\right)^{3+} \\
5.21\end{array}$ & $\begin{array}{l}F e^{+3}\left(d^{5}\right) \\
5.92\end{array}$ & High & 5.52 \\
\hline & 5.61 & 1.78 & 1.73 & Low & 2.10 \\
\hline
\end{tabular}

We note that the above examples are possible outcomes but not a definitive model. The XPS data show a clear increase in binding energy of Fe and Co ion and a downshift in binding energy for the amine and carboxylate bonds. We expect the charge transfer to occur in the amine group of FF-MNS. Shown in Figure 5.19, is a segment of the FF macrocycle where two neighboring units form a zwitterion; the carboxylic group is negatively charged, and the amine group is positively charged. Although the charge transfers to the amine group but since the carboxylic group is connected to it, changes in the vibrational frequencies associated with both groups are expected, as observed in the FTIR spectra. Figure 5.19 shows a schematic of the $\mathrm{Co}$ and $\mathrm{Fe}$ ions in the $\mathrm{T}_{\mathrm{d}}$ and $\mathrm{O}_{\mathrm{h}}$ sites that are most likely involved in the charge transfer process. 


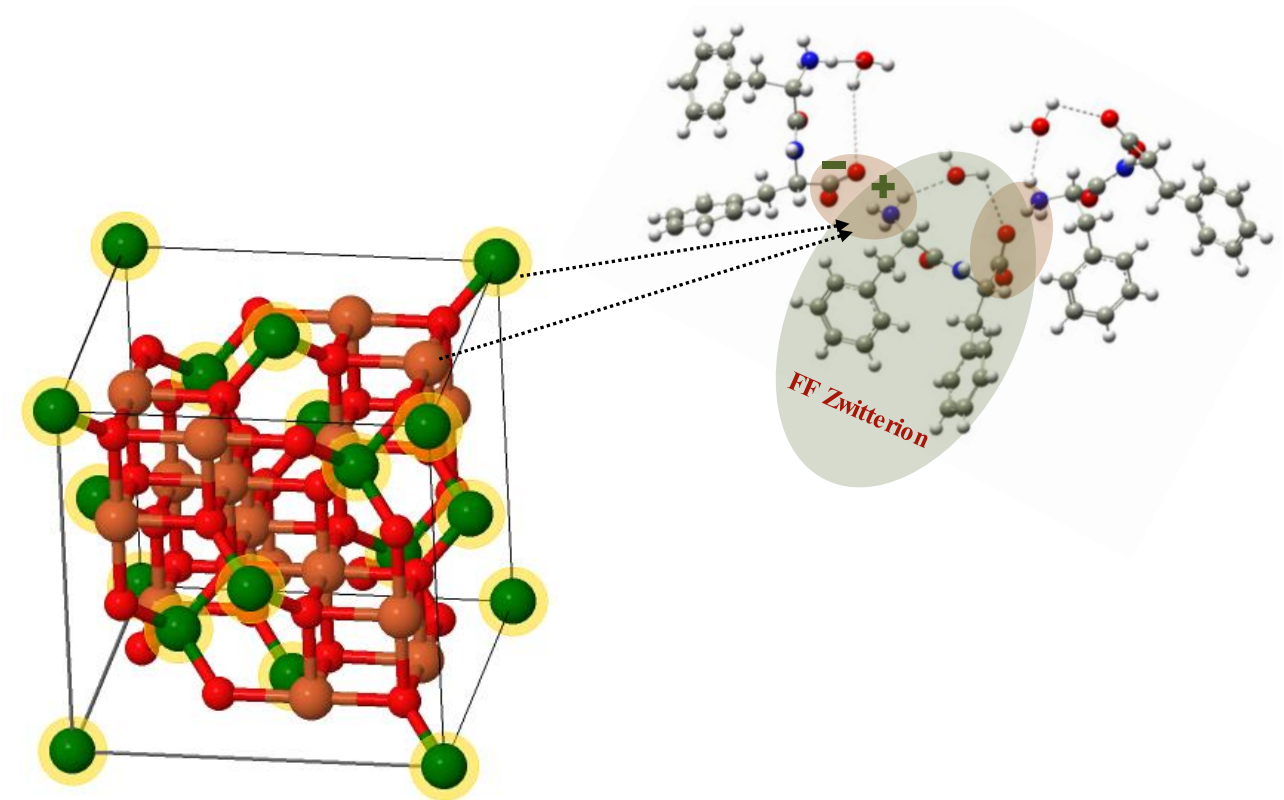

Figure 5.19. Schematic of the interaction model between CFO and the amine group in FFMNS.

\subsubsection{Application of FF-MNS:CFO in Organic Electronics}

Organic FETs are being actively developed for applications in large-area flexible electronics. ${ }^{116-117}$. Appropriate gate dielectrics that are free of hydroxyl or silanol are necessary for improving charge transport. In order to achieve low-operating voltage FETs, the dielectric surface should be hydrophobic and must provide a relatively high capacitance, which is often a challenge with many polymer dielectrics mainly due to their inherently low dielectric constant. FF-MNS has been successfully used as a dielectric layer, both as a bottom-gate and top-gate, in pentacene FETs $;{ }^{7}$ the nanostructure morphology of FF-MNS was shown to be responsible for withstanding a bias-stress effect. Since CFO has good dielectric properties, we used the FF-MNS:CFO nanocomposite sample both in pentacene MIS capacitors and FETs. 
(a)
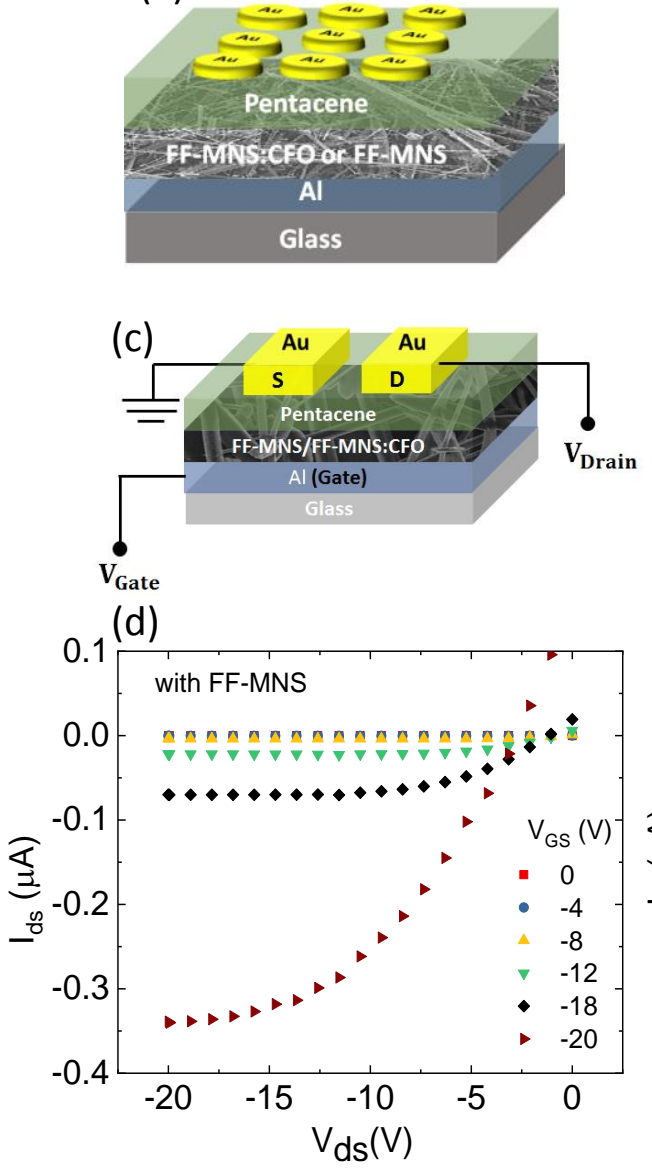

(b)

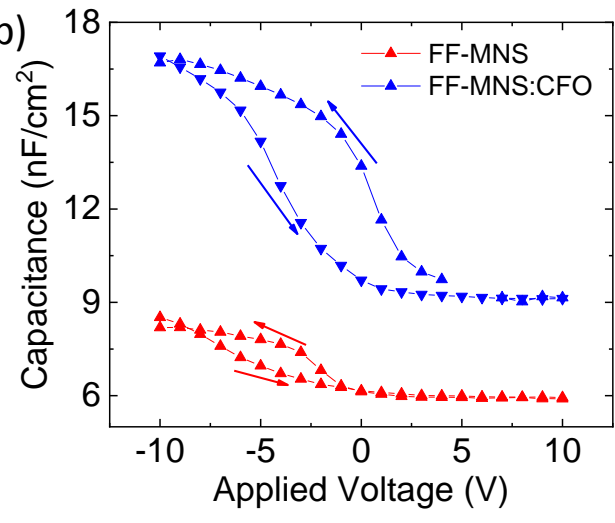

(e)

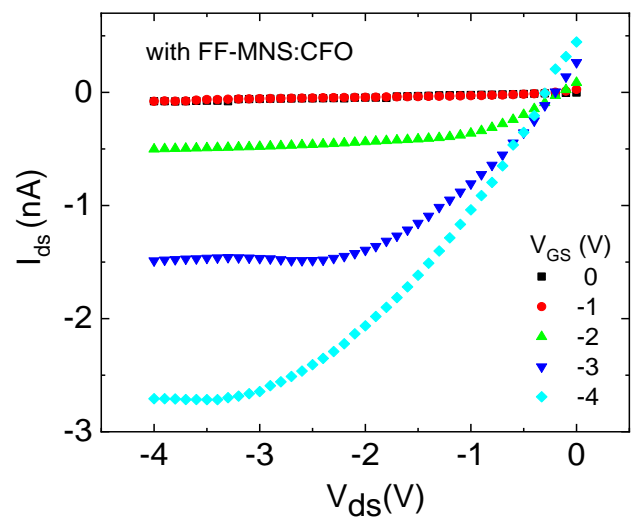

Figure 5.20. Electrical characteristics of pentacene based MIS capacitors and FETs. FFMNS and FF-MNS:CFO served as the dielectric layer. (a) Schematic of MIS structures. (b) C-V characteristics of MIS devices. The arrows denote the sweep directions. (c) Schematic of a bottom-gate, top-contact FET. (d) and (e) Output characteristics of pentacene FETs using FF-MNS and FF-MNS:CFO as the gate dielectric, respectively. The channel width to length ratio of the FETs was 10.

Figure 5.20 (a) shows a schematic of the MIS structure with pentacene as the semiconducting layer, and FF-MNS or FF-MNS:CFO as the dielectric layer. The capacitance versus voltage $(\mathrm{C}-\mathrm{V})$ curves, in Figure 5.20 (b), show a typical p-type behavior with an accumulation of holes at the semiconductor/dielectric interface at negative bias, a depletion, and a deep depletion region. This is similar to the $\mathrm{C}-\mathrm{V}$ behavior from MIS capacitors utilizing pentacene and other dielectrics. ${ }^{118-119}$ The film thicknesses of FF-MNS and FF-MNS:CFO were approximately the same $(550 \mathrm{~nm})$. An increase in the overall 
capacitance is clearly observed when FF-MNS:CFO is used. Since the film thicknesses of FF-MNS and FF-MNS:CFO were approximately the same $(550 \mathrm{~nm})$, the enhanced capacitance in the nanocomposite sample originates due to the presence of CFO. Hysteresis in $\mathrm{C}-\mathrm{V}$ curves have various origins due to interface charges and slowly polarizing dipole charges, and is typical for polymer dielectric thicknesses greater than $50 \mathrm{~nm} .{ }^{119}$

Since organic FETs operate in the accumulation region, the operating voltage depends on the charge density. Given $Q=C_{i} V_{g s}$, where $C_{i}$ is the capacitance of the insulator and $V_{g s}$ is the gate-source voltage, a higher value of $C_{i}$ would imply low operating voltage FETs. To test this, we fabricated pentacene FETs using FF-MNS and FF-MNS:CFO as bottom-gate, top-contact architectures, schematically shown in Figure 5.20 (c). A thin layer $(<10 \mathrm{~nm})$ of poly(4-vinyl phenol) was deposited below the FF-MNS or FF-MNS:CFO layer to reduce the gate leakage current. The output curves (with different gate voltages) from the two FETs are shown in Figure 5.20 (d); the FF-MNS:CFO based FET shows a lower operating voltage by a factor of 5 compared to the FF-MNS FET. The magnitude of the drain-source current $\left(\mathrm{I}_{\mathrm{ds}}\right)$ is different due to differences in the leakage current. A study using CFO nanoparticles in a floating gate memory device shows that the average FET carrier mobility is small, mainly due to leakage effects, when the size of the nanoparticles is large; the size of the CFO nanoparticle in this study was varied between $5-11 \mathrm{~nm} .{ }^{85} \mathrm{We}$ point out that in our case the size of the CFO nanoparticles is $\sim 30 \mathrm{~nm}$.

Although the FET application does not exploit the enhanced magnetic properties of $\mathrm{CFO}$ in the nanocomposite sample, it provides a general route for lowering operating voltages for other peptide nanostructures or polymer dielectrics to be used as a gate dielectric layer. Moreover, the magnetic properties may provide a potential tuning knob for 
changing the electrical properties in organic FETs or MIS capacitors in the presence of an external magnetic field.

\subsubsection{Summarization of Functionalization of CFO with FF-MNS}

Functionalization of bioinspired peptide nanostructures, FF-MNS, with magnetic nanoparticles of CFO provides an attractive path towards enhancing the magnetic properties. This may find several applications in electronics or in biomedicine where CFO could be contained in a benign environment. The magnetization behavior of both CFO nanoparticles and FF-MNS:CFO follow a modified Langevin-type behavior, characteristic of superparamagnetism. The enhanced saturation magnetization of the nanocomposite sample over pristine CFO sheds insight into the cation distribution and the nature of charge transfer. The inverse spinel structure of CFO with Fe and Co ions both in the $\mathrm{O}_{\mathrm{h}}$ and $\mathrm{T}_{\mathrm{d}}$ site provides interaction sites with the amine group in FF-MNS, where either the Co ions or both Fe and Co ions give up an electron to the amine group.

A combination of structural and spectroscopic investigations was performed to probe the mechanism of charge transfer between the peptide nanostructure and CFO. XPS is a sensitive technique for elucidating the oxidation state of elements. An enhancement in the binding energy of $\mathrm{Fe} 2 \mathrm{p}_{3 / 2}$ and Co peaks in FF-MNS:CFO compared to pristine CFO in the XPS spectrum denotes a charge transfer to FF-MNS. The CFO Raman peak at $675 \mathrm{~cm}^{-1}$ hardens by almost $10 \mathrm{~cm}^{-1}$; concomitantly the amide I and amide V vibrations in FTIR are seen to soften in FF-MNS:CFO, which is attributed to a charge transfer from $\mathrm{Fe}^{2+} / \mathrm{Co}^{2+}$ ions to the amine group. Since the carboxyl group is in the proximity of the amine group, the $\mathrm{C}=\mathrm{O}$ vibrations from FF-MNS in FTIR are also affected. 
The dielectric properties of FF-MNS and FF-MNS:CFO were exploited in pentacene MIS capacitors and FETs. A higher value of capacitance in FF-MNS:CFO compared to FF-MNS, observed in MIS capacitors, is responsible for lowering the operating voltages in pentacene FETs. These results have ramifications in low operating voltage floating gate memory FETs. The integration of peptide nanostructures with magnetic nanoparticles opens up a new generation of bioinspired hybrid materials for application in organic electronics. Future strategies will involve functionalizing FF- and other short peptides with amphiphilic entities with hydrophobic tails for improving the interaction between selfassembled peptide nanostructures and magnetic nanoparticles. 


\section{$6 \quad$ FF-MNS as Templates for Organic Light Emitting Diode (OLED)}

The goal of this chapter is to utilize FF-MNS as a scaffold for OLED. Functionalized FF-MNS is used as biodegradable active layer in an organic OLED. FF-MNS was functionalized with two blue emitting conducting polymers: di-octyl-substituted and ethylhexyl polyfluorene (PF2/6). A combination of molecular dynamics and experimental characterization techniques reveal a stronger binding mechanism for PF8 compared to PF2/6 with FF-MNS. Enzyme based tests confirm the biodegradability of $80 \%$ of the FFMNS:PF8 nanocomposite films. This study opens up a new generation of biocompatible and biodegradable materials in organic electronics.

\subsection{Introduction}

The efficacy of FF-MNS as a dielectric layer in bottom-gate and top-gate pentacene field-effect transistors (FETs) has been shown.120 The field of organic semiconductors has rapidly grown with complementary options to inorganic semiconductors in electronic devices, providing flexibility and large-area applications. However, there remains a challenge in controlling the structure and morphology of organic semiconductors at the nanoscale level, purely by synthetic routes. Conjugated polymer-based nanostructures have unique properties that may be harnessed in optical communications, solid-state lighting, and laser applications. ${ }^{121-122}$ Most of the nanostructuring processes for conjugated polymers require expensive nanopatterning techniques with multiple steps. ${ }^{123-124}$ Peptide selfassembly has been utilized for creating functional nanoscale $\pi$-conjugated molecules and oligomers. ${ }^{125}$ Specifically, such systems have involved peptide- $\pi$-peptide self-assembly for providing electronically delocalized supramolecular structures. ${ }^{126}$ However, these 
supramolecular structures require involved synthesis routes for both peptides as well as the conjugated molecules/oligomers.

Taking advantage of the $\pi$-stacking interaction between FF-MNS and fluorophores such as pyrenil and hypericin, it has been shown that peptide self-assembly promotes adsorption of molecules, yielding functional nanocomposites. ${ }^{127-128}$ Since the synthetic routes for such nanocomposites are straightforward, it has motivated us to explore the selfassembly of FF-MNS with conjugated polymers for possible application in organic electronics. In such structures, the charge transport still occurs through the conjugated polymers; the FF-MNS mainly provide a scaffold for the organic semiconductors along with the prospect of obtaining nanostructures for the polymer itself. Well-defined spatial arrangements can be achieved by peptide self-assembly and may be exploited to position aromatic ligands in optimal positions for an effective $\pi$-orbital overlap. Additionally, due to the ease of manipulating FF-MNS on active substrates for electronics, one can conceive of new functionalities in organic semiconductor-based devices.

Blue Light Emitting Polyfluorenes: Polyfluorenes (PFs) are highly efficient blue light emitting $\pi$ conjugated polymers. Device performance based on this polymer depends on side chain conformations, overall crystalline structure, and charge transport processes at the microscopic level. In the solid state, PFs show the highest photoluminescence quantum yield (PLQY) amongst conjugated polymer. ${ }^{129}$

The monomer unit consists of rigid planar biphenyl units, bridged by a carbon atom ensuring a high degree of conjugation, shown in Figure 6.1 (a). Electrical and optical properties of PF derivatives strongly depend on the substituents at the bridging carbon. In this work we have used two types of polyfluorenes having different two side chain 
substitution PFs: Poly(2,7-[9,9'-bis(2-ethylhexyl)] fluorene) (PF2/6) and Poly(9,9-(din,noctyl) fluorene) (PF8), as shown in Figure 6.1 (b), (c). In the solid state, PF2/6 assumes a 21-helical main chain conformation, resulting in a hexagonal phase which self-organizes into a three-chain unit cell. ${ }^{130}$ PF8 shows many crystalline phases and conformations that depend upon the thermal history and the nature of the solvent. ${ }^{131}$ The alkyl side chains in PFs give rise to a rich array of mesomorphic behavior with the appearance of the nematic liquid crystalline phase (n-LC) at temperatures above $160^{\circ} \mathrm{C}$.

(a) Polyfluorene

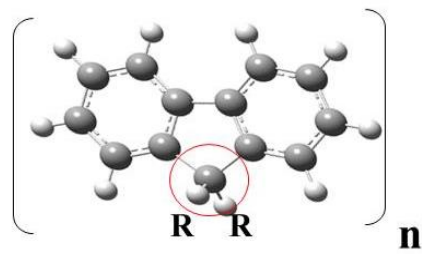

(b)

$$
\text { PF2/6 }
$$

\section{$\mathbf{R}=$ 2-ethylhexyl}

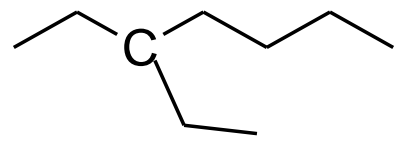

(c)

$$
\mathrm{R}=\mathrm{n} \text {-octyl }
$$

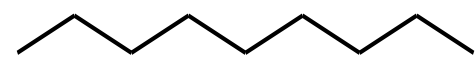

Figure 6.1. (a) Monomer unit of Polyfluorene. (b) Side chain of PF2/6. (c) Side chain of PF8.

PF8 is assessed both in terms of its overall crystalline phase as well as its conformational isomers. There are at least three different conformational phases in PF8 which depend on the backbone torsional angle. The most planar conformation, $\mathrm{C}_{\beta}$, also referred to the beta phase, is characterized by a torsional angle of $165^{\circ 131}$ between adjacent monomer units. Although the beta phase may be present in small quantities, being a low energy state, it dominates the optical emission. The beta phase can be promoted by aging the polymer in solvents such as toluene.

In Figure 6.2, the Photoluminescence emission from the two polyfluorenes are shown. They show typical vibronic structures seen other conjugated polymers. The peak highest in energy corresponds to the 0-0 peak, followed by the 0-1 and 0-2 peaks. 

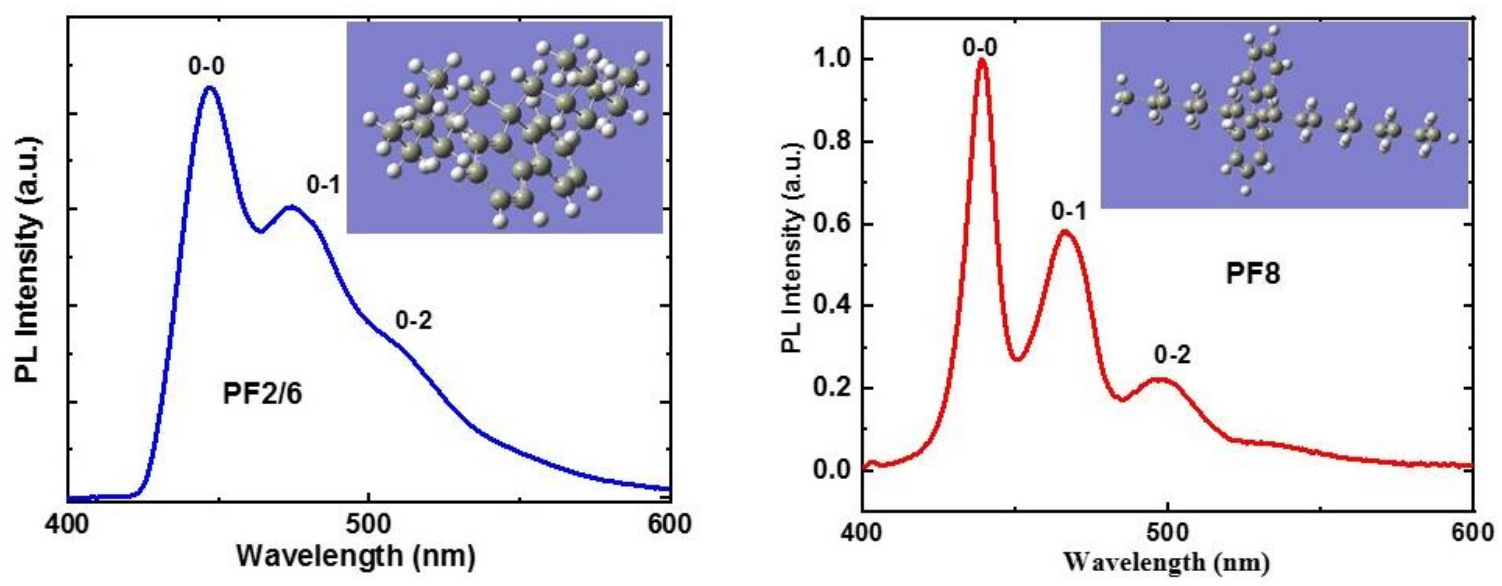

Figure 6.2. PL spectra of (a) PF2/6 and (b) PF8.

\subsection{Synthesis of Peptide-polyfluorene Nanocomposite}

Peptide-polyfluorene nanocomposites were synthesized using a liquid vapor process, ${ }^{132,16}$ shown in Figure 6.3. In this method, $100 \mu \mathrm{L}$ of FF from HFIP solution (100 $\mathrm{mg} / \mathrm{mL}$ ) was mixed with $1800 \mu \mathrm{L}$ of polyfluorene (PF2/6 or PF8) from DCB solution (10 $\mathrm{mg} / \mathrm{mL}$ ) and $100 \mu \mathrm{L}$ of DI water. This mixture was immediately ultra-sonicated for about a minute at $10 \mathrm{kHz}$ frequency. The mixture was then kept in the refrigerator for about 8 hours for the reaction to occur between the FF and PFs. The mixture was then centrifuged to separate the solid and liquid phase. The liquid phase was removed by a pipette from the separated solid-liquid mixture and the solid phase was kept in an oven at $75^{\circ} \mathrm{C}$ until dry. In the case of the aged PF8 sample, PF8 was first dissolved in toluene $\left(10 \mathrm{mg} \mathrm{mL}^{-1}\right)$ and left at $-20{ }^{\circ} \mathrm{C}$ for $60 \mathrm{~h} .{ }^{133}$ The same procedure as above was followed for synthesizing FFPF8 using aged PF8, except toluene was used instead of DCB.

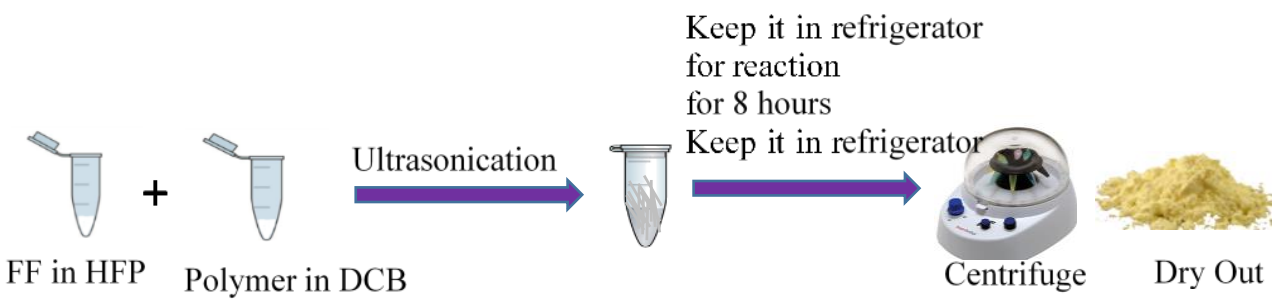

Figure 6.3. Liquid vapor process. 


\subsection{Interaction Study of the Polymer with Peptide Nanostructure using SEM,}

\section{TEM, PL, Absorption Measurement and MD Simulation}

Understanding the nature of FF-MNS-polymer interaction is critical for applications. Using these nanocomposites as electronic materials, it is necessary to know how charge transport occurs and whether the polymer binds to the nanostructures or not. Investigations of the interaction between PF and FF-MNS were performed via TEM/SEM, optical spectroscopy (absorption, PL, electroluminescence (EL), ) and molecular dynamics (MD) simulations.

\section{TEM/SEM}

For TEM, specimens were diluted in isopropanol, dispersed on a Protochips C flat holey carbon film patterned with 1 micron holes on copper 200 mesh TEM grid and allowed to dry. TEM was performed with an FEI Tecnai F30 Twin operated at $300 \mathrm{kV}$ with spot 3. Images were acquired on a Gatan Ultrascan 4000 CCD camera.
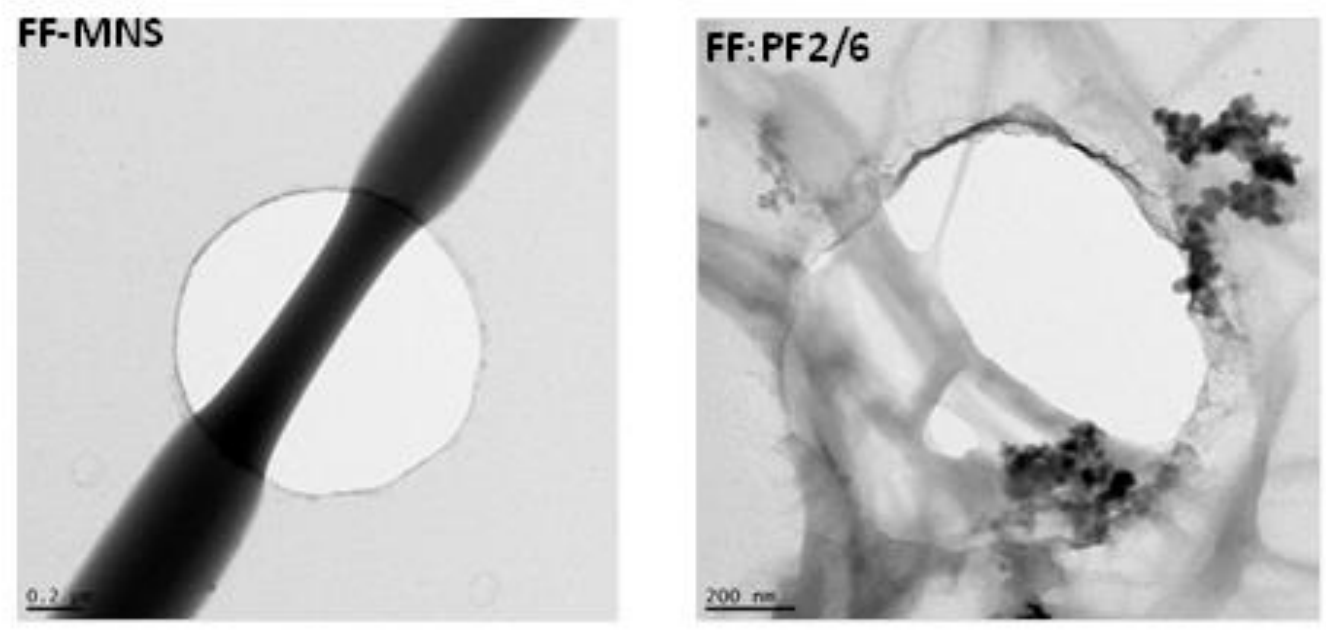

Figure 6.4. TEM image of (a) a FF nanotube and (b) FF:PF2/6. The dark regions in (b) show the aggregation of $\mathrm{PF} 2 / 6$. 
(a)

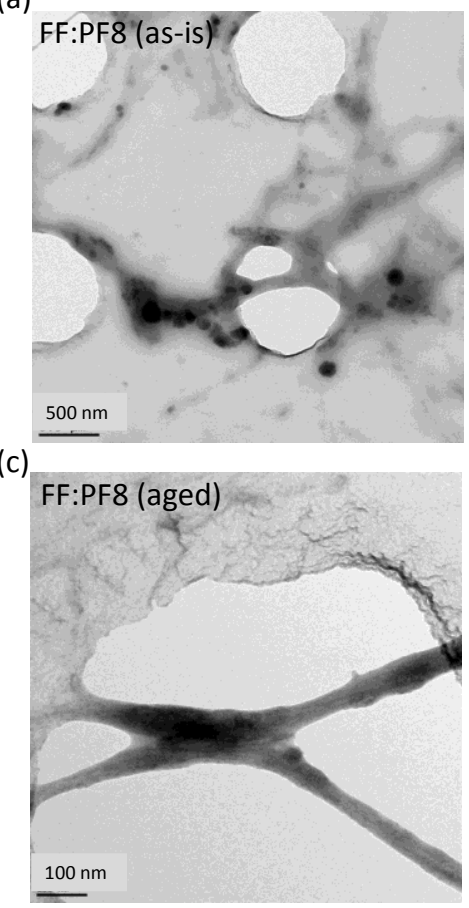

(b)

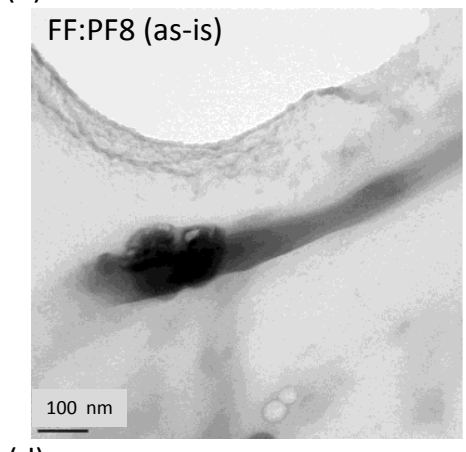

(d)

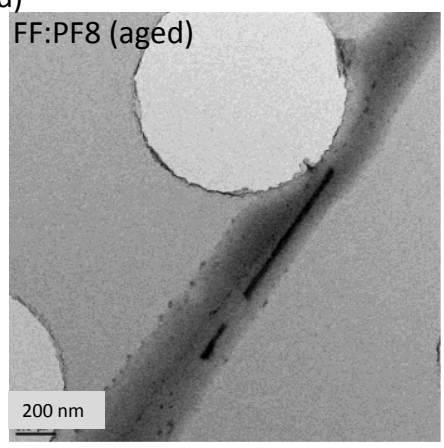

Figure 6.5. (a) TEM image of FF:PF8 (as-is) nanocomposite. (b) A magnified image of a FF:PF8 (as-is) nanotube. (c) and (d) are representative TEM images of FF:PF8 (aged) nanotubes. In all images, the dark regions are the polymer and the lighter rod-like structure is the FF nanotube.

Figure 6.4 (a) shows the TEM image of a FF nanotube and Figure 6.4 (b) shows the TEM image of FF: PF2/6. Figure 6.5 shows the TEM images of both FF: PF8 (as is) and FF: PF8 (aged). FF:PF8 (as is or aged) images are quite different from FF: PF2/6 signifying better binding of PF8 to the FF tubes. The PF8 (as-is) also seems to coalesce over 50-100 nm length scales. Figure 6.5 (b) shows the magnified image of Figure 6.5 a showing both the aggregation and binding of PF8 to the FF nanotube. Since aged PF8 forms nanofibers, the TEM images from FF:PF8 (aged) sample (Figure 6.5 (c), (d)) show a better binding of PF8 nanofibers with FF nanotubes, compared to as-is PF8 with FF. For some images, the elongated nanofibers morphology of PF8 polymer is seen on top of the FF nanotube. As a further step towards understanding the interaction of PF8 with FF-MNs, we have conducted 
detailed optical spectroscopic studies from both FF:PF8 (as-is) and FF:PF8 (aged) nanocomposites.

\section{Absorption and PL}

Figure 6.6 (a), (b), and (c) show the absorption spectra PF2/6, PF8, aged PF8 and their composites with FF-MNS. Figure 6.6 (d), (e), and (f) are the PL spectra from PF2/6, PF8, aged PF8 and their composites with FF-MNS. We point out that the films were drop casted for the PL and absorption measurements. The $435 \mathrm{~nm}$ shoulder in the absorption spectra is the signature of the beta phase, indicating that the as-is polymer has some fraction of this phase, Figure $6.6(\mathrm{c})$. During the nanocomposites sample preparation, there is an additional promotion of the beta phase. PF8 (aged) has a higher fraction of the beta phase (Figure 6.6 (c)), as seen from the higher intensity of the $435 \mathrm{~nm}$ peak in the absorption spectrum, when compared to PF8 (as-is). The absorption spectra of FF:PF8 (aged) and PF8 (aged) are quite similar.
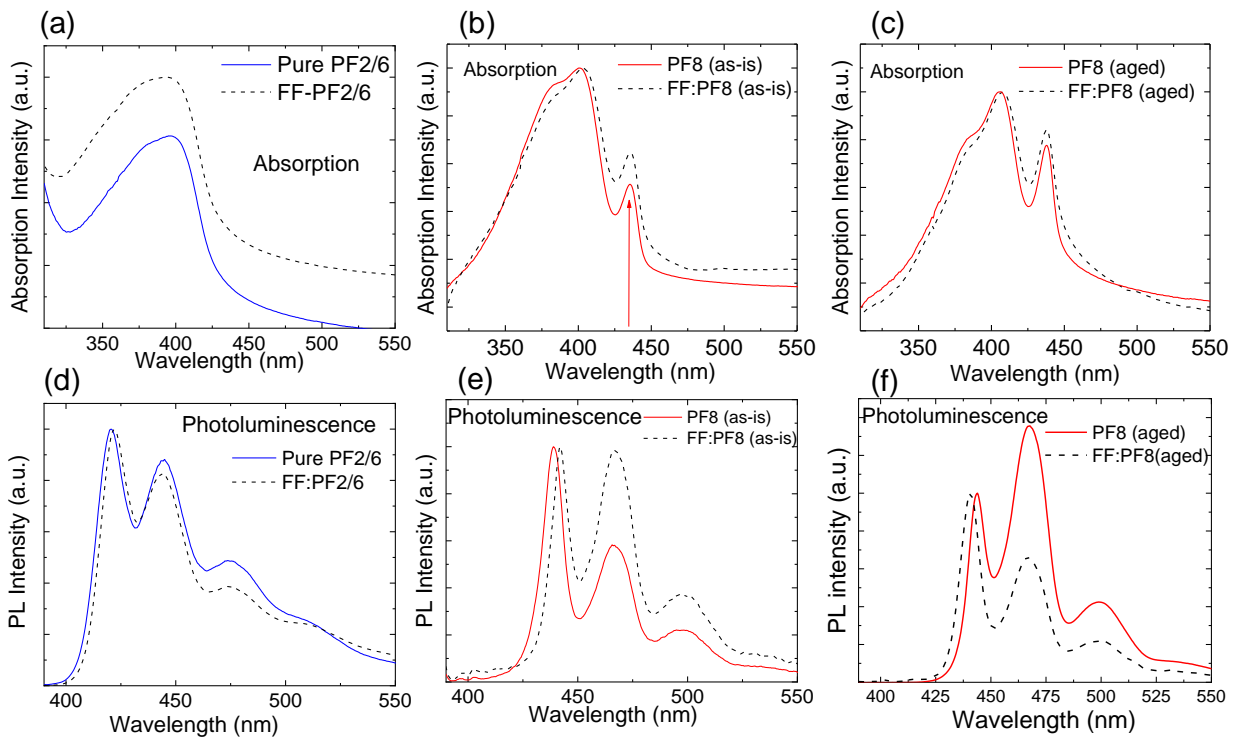

Figure 6.6. Absorption spectra of (a) PF2/6 and FF:PF2/6, (b) PF8 and FF:PF8 (as is), and (c) PF8 (aged) and FF: PF8(aged) (The peak at $435 \mathrm{~nm}$ depicted by the red arrow is a signature of the beta phase). PL spectra of (d) PF2/6 and FF:PF2/6, (e) PF8 and FF:PF8 (as is), and (f) PF8 (aged) and FF: PF8(aged). 
Similar to PF2/6 (Figure 6.6 (d)), the PL spectrum shows a vibronic progression; the 440 and $466 \mathrm{~nm}$ peaks in as-is PF8 are the $0-0$ and $0-1$ transitions, respectively (Figure 6.6 (e)). Since the optical density is high and the PL spectrum is not corrected for selfabsorption effects, the $0-1$ peak shows a higher intensity compared to the $0-0$ peak in aged PF8 (Figure $6.6(\mathrm{f})$ ). As the wt $\%$ of PF8 is much lower in the nanocomposite film compared to the pristine PF8 film, the self-absorption effect is low and the $0-0$ PL peak shows a higher intensity compared to the $0-1$ peak in FF:PF8(aged). We note that there is a redshift of the PL spectrum of FF:PF8 (as is) compared to the pristine polymer. The redshift may be attributed to two effects: a higher interaction of PF8 with the FF nanotubes, which further promotes a nanostructuring effect of the polymer itself, and due to the formation of additional beta phase chromophores.

\section{Simulations}

MD simulations were performed with monomers of the two PF polymers and FF nanotubes. Two simulations were performed in order to investigate the interaction between the surface of the FF nanotube (FFNT) and the conjugated polymers: PF2/6 and PF8. Since the entire system is quite large to handle, only the monomers were used in the MD simulations. To a first approximation since the PF monomers have distinct alkyl side chains, the MD simulations allow a meaningful insight into their respective interactions with FFNT. 


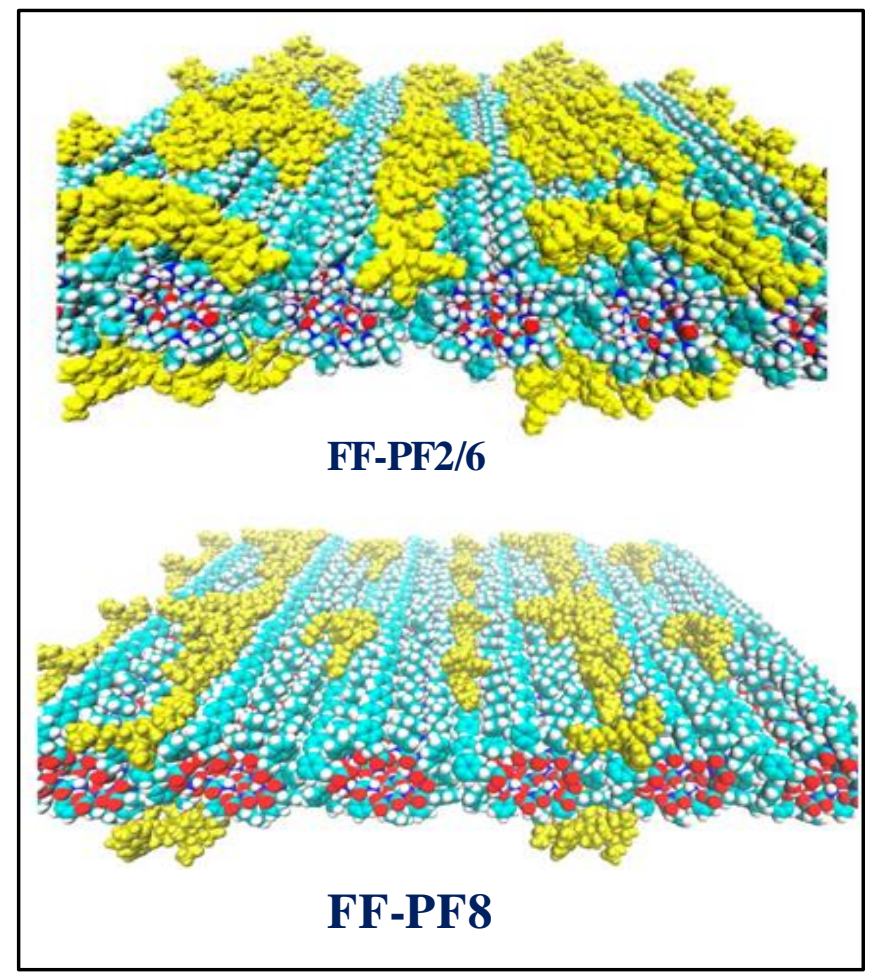

Figure 6.7. (a) Panoramic view of the distribution of PF2/6 and PF8 monomers on nanotubes surface.

Table 6.1. Interaction energy of two nanocomposites.

\begin{tabular}{llll}
\hline Molecule & $\mathrm{E}_{\text {Total }}\left(\mathrm{kJmol}^{-1}\right)$ & $\mathrm{E}_{\mathrm{vdW}}\left(\mathrm{kJmol}^{-1}\right)$ & $\mathrm{E}_{\mathrm{Coul}}\left(\mathrm{kJmol}^{-1}\right)$ \\
\hline PF2/6 & $\mathbf{- 6 0 . 8} \pm \mathbf{2 . 0}$ & $\mathbf{- 6 2 . 9} \pm \mathbf{2 . 0}$ & $\mathbf{2 . 1} \pm \mathbf{0 . 1}$ \\
PF8 & $\mathbf{- 6 7 . 5} \pm \mathbf{2 . 3}$ & $\mathbf{- 6 9 . 7} \pm \mathbf{2 . 3}$ & $\mathbf{2 . 2} \pm \mathbf{0 . 1}$ \\
\hline
\end{tabular}

Figure 6.7, shows a panoramic view of PF8 and PF2/6monomers on the nanotube surface. We clearly see the adsorption pattern of the PF8 molecules that form aggregates and spread over the rough nanotube surface. This pattern is expected to depend on experimental conditions, particularly the concentration of polymer employed. Considering the hydrophobic nature of PFs as well as the nanotube surface, one would expect this aggregation feature of the adsorption process. PF2/6 monomers show a similar feature on FF-MNSs 
The magnitude of the interaction strengths of the adsorption process can be partially estimated by the energy of interaction between polymer molecules and nanotube surface. The interaction total energy $\left(\mathrm{E}_{\text {Total }}\right)$, per polymer molecule, was calculated and decomposed into electrostatics $\left(\mathrm{E}_{\mathrm{Coul}}\right)$ and van der Waals $\left(\mathrm{E}_{\mathrm{vdW}}\right)$ components. The interaction strengths are governed by van der Waals energy: -63 and $-70 \mathrm{~kJ} \mathrm{~mol}^{-1}$ per PF2/6 and PF8 molecule, respectively. This energy is relatively large, making the electrostatic component $(\approx 2 \mathrm{~kJ}$ $\mathrm{mol}^{-1}$ ) negligible. The energies are tabulated in Table 6.1. We note that the interaction of PF8 with the FFNT surface is higher than PF2/6. Although both PF molecules have a similar chemical structure albeit with different side-chain substitution, PF8 favors greater proximity to the grooves in the surface of the nanotube, resulting in a higher intermolecular interaction compared to $\mathrm{PF} 2 / 6$.

\subsection{Composite Based OLED}

The primary goal in fabricating the EL devices is not to quantify the luminescence efficiency, but rather to see if there are any shifts in the spectrum between pristine and nanocomposite films. This would be an indicator of some kind of an interaction between the polymer and FF-MNS. FF-MNS:PFs were used as active layer in OLED structure.
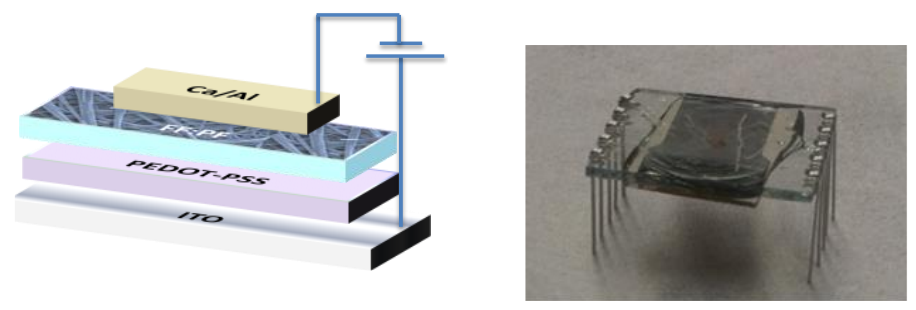

Figure 6.8. (a) shows the architecture of the OLED.(b) Image of a OLED device fabricated in our lab.

A filtered (using polyvinyl difluoride $0.45 \mathrm{~mm}$ ) poly $(3,4$-ethylenedioxythiophene) poly(styrenesulfonate) (PEDOT:PSS) solution was spun cast onto cleaned ITO glass 
substrates at $5000 \mathrm{rpm}$ for $40 \mathrm{~s}$ and then baked at $120{ }^{\circ} \mathrm{C}$ for 25 min under an $\mathrm{N}_{2}$ atmosphere. FF:PF2/6 and FF:PF8 (aged) were dissolved in DCB $\left(10 \mathrm{mg} \mathrm{mL}^{-1}\right)$. The solution was spincoated at $700 \mathrm{rpm}$ for $40 \mathrm{~s}$ on top of the PEDOT-PSS layer and then baked at $90{ }^{\circ} \mathrm{C}$ for 20 min under an $\mathrm{N}_{2}$ atmosphere. For the pristine PF films, a similar ratio was used (10 mg $\mathrm{mL}^{-1}$ (DCB). The thickness of the active layer was about $100 \mathrm{~nm}$ and the device area was $0.045 \mathrm{~cm}^{2}$. The top $\mathrm{Ca}-\mathrm{Al}$ electrode was deposited by a thermal evaporator at base pressure of $10^{-5}$ mbar. The diode structure was encapsulated using UV curable material (AC A1430B) after thermal evaporation of the top electrode. All fabrication steps were carried out inside a nitrogen glove box.
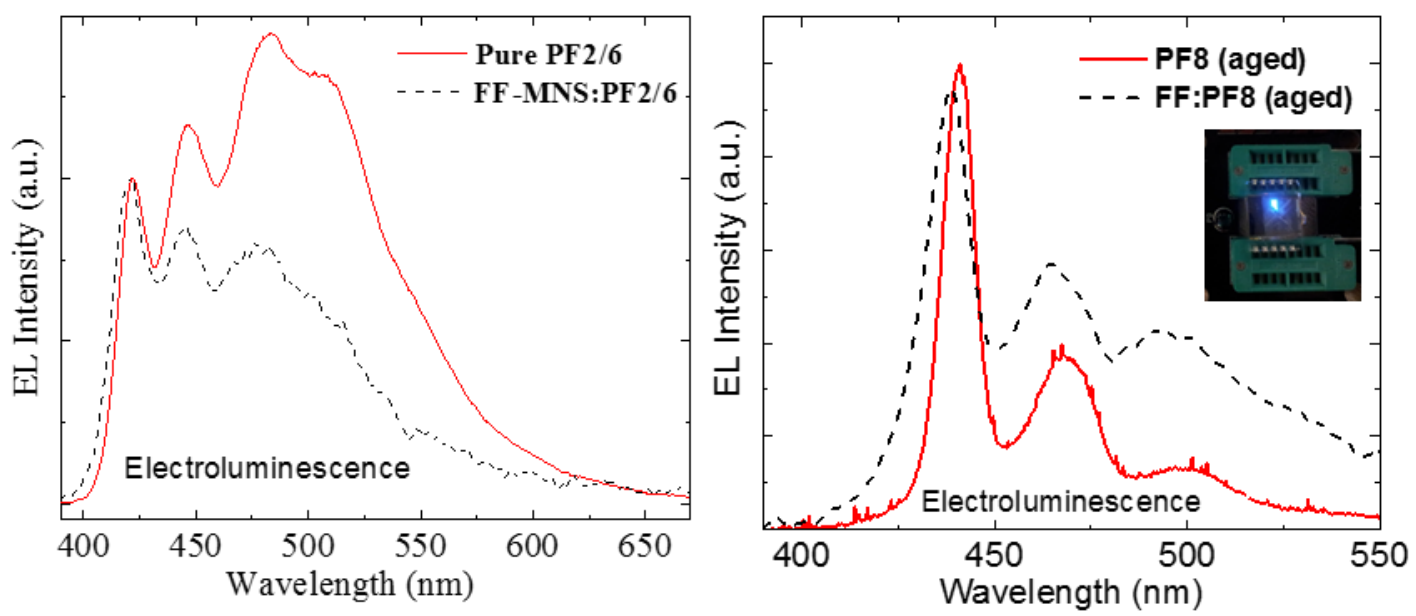

Figure 6.9. EL spectra of (a) PF2/6 and FF-MNS:PF2/6, and (b) PF8 and FF-MNS:PF8.

OLED structures were fabricated from PF2/6, FF:PF2/6, PF8 (aged) and FF:PF8 (aged) films. Figure 6.9 compares the EL spectra from these devices. EL spectra were collected by applying an external voltage to device. Comparing the EL spectra of the pure polymer with that of the composite is another measure of gauging the interaction between the polymer and the FF-MNS. 
The overall intensity from the nanocomposite OLED is roughly six times less than the pristine PF8 device. It is interesting to note the slight blue shift of the EL spectrum of the nanocomposite device compared to the only PF8 (aged) device. There was no shift in the EL spectra from FF:PF2/6 nanocomposite device compared to pure PF2/6 device. The PL spectra (Figure 6.6 (f)) of the two films show a similar trend-the FF:PF8 (aged) film is slightly blue shifted compared to PF8 (aged) film. Since the $0-0$ and the $0-1$ vibronics both blue shift in FF:PF8 (aged), it could indicate a deviation from the planar backbone conformation in PF8 upon interacting with FF-MNS. The two polymers, PF2/6 and PF8, interact with the FF nanotubes differently. Both electron microscopy and the optical data indicate that PF8 (as-is and aged) interacts more strongly with FF-MNS compared to $\mathrm{PF} 2 / 6$.

\subsection{Biodegradability Test}

In recent years, enzyme-mediated degradation has been investigated for polymeric biomaterials. ${ }^{134}$ To test the efficacy of such degradation, FF:PF8 nanocomposite, FF-MNS, and PF8 films are tested with proteinase $\mathrm{K}$, a protease which hydrolyses amide bonds. The weight loss of FF:PF8 film over 2 hours is almost $80 \%$ in contrast to only PF8 films, which shows no enzymatic degradation, thus paving the way for biodegradability of the active layer in organic electronics.

To test the biodegradability of the nanocomposite film, pure FF-MNS, FF:PF8, and PF8 films were drop-casted on glass slides. The films were weighed before incubation in a buffer/enzyme solution at $37^{\circ} \mathrm{C}$. At different time intervals, the films were removed from the culture, vacuum dried till there was no mass change, and then weighed again. The percentage of weight loss is given by $\frac{m_{\text {after }}-m_{0}}{m_{0}}$ where $m_{0}$ is the vacuum dried weight after 
each incubation time period and $m_{0}$ is the initial dried weight of each sample. Since the enzymatic activity is lost after $24 \mathrm{~h}$, we monitored the weight loss only over a $24 \mathrm{~h}$ period. Figure 6.8 is a plot of the weight loss (\%) for each of the samples. It is seen that within 2 h, there is more than $80 \%$ weight loss for the FF-MNS and FF:PF8 films. In contrast, the PF8 film remains unchanged.

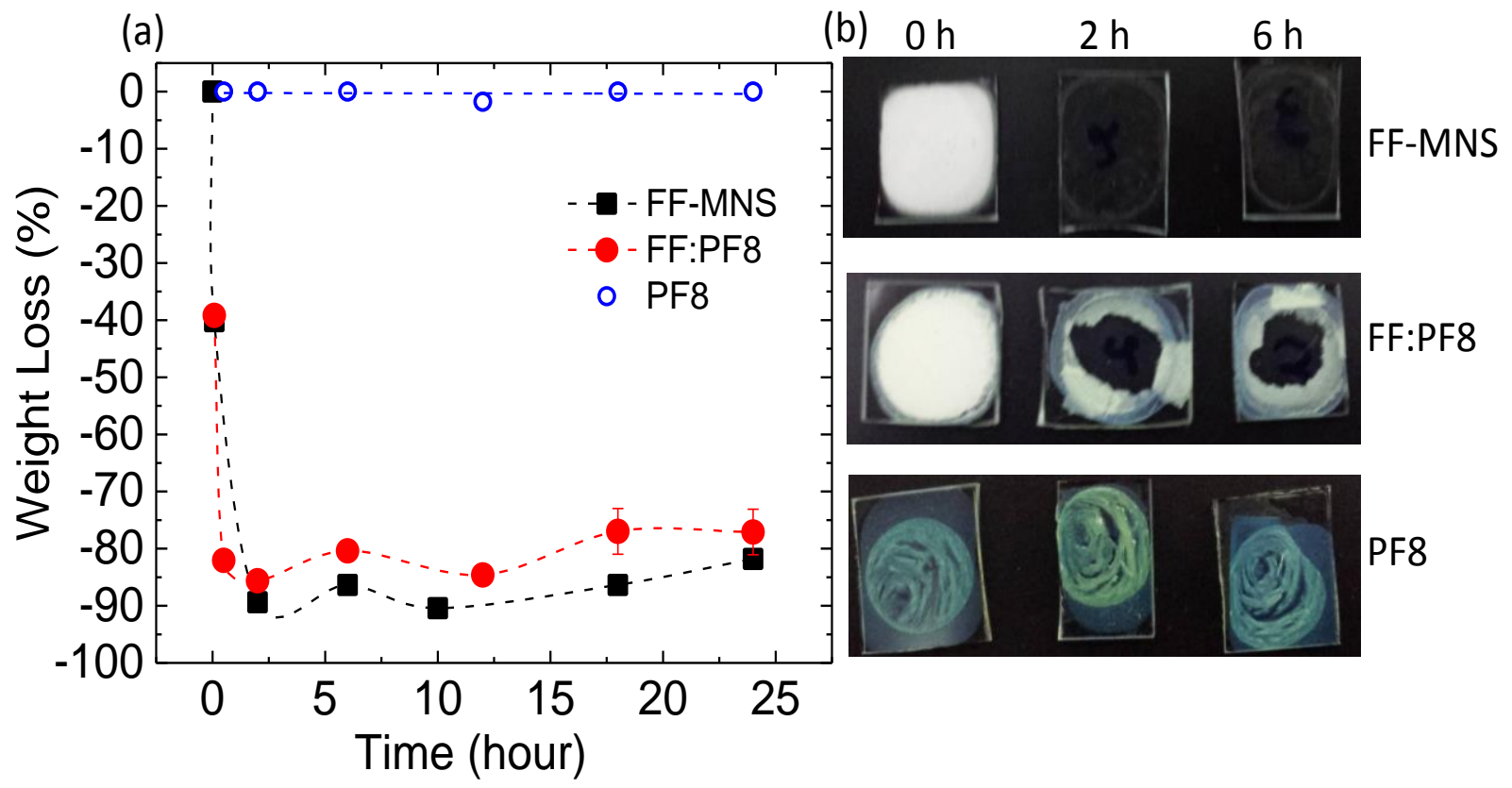

Figure 6.10. a) Percentage weight loss of FF-MNS, FF:PF8, and PF8 after incubation in buffer/enzyme system at $37{ }^{\circ} \mathrm{C}$ at different time intervals. b) Optical images of the three films before and after 2 and $6 \mathrm{~h}$ of incubation in buffer/enzyme solution.

The use of FF-MNS as scaffolds for conjugated polymers opens up the possibility of organic optoelectronics, where the active layer itself is biodegradable. It has already been demonstrated that biodegradable substrates such as cellulose may be used both as substrates for LEDs, displays, and thin-film transistors. ${ }^{135} \mathrm{~A}$ recent study shows the usage of cellulose based dielectrics in high-performance organic complementary inverters. ${ }^{136}$ Additionally, FF-MNS, which is almost fully biodegradable, has also been demonstrated as a dielectric layer in top- and bottom-gate organic FETs. ${ }^{120}$ It is conceivable that all the 
layers, including the electrodes which could be water-based inks, in organic electronic devices are not just printable but also biodegradable in the very near future.

\subsection{Concluding Remarks}

FF-MNS as templates for conjugated polymers such as PFs open up a new route toward bottom-up scalable processes for organic electronics. Owing to the nanostructuring effect of FF peptides, the surface area of the active polymer is greatly enhanced in FF:PF nanocomposites. This is economical in electronic devices since a small wt $\%$ of the polymer can result in similar device performance compared to only conjugated polymer films as the active layer. By using two side-chain substituent polyfluorenes, PF8 and PF2/6, with FFMNS, we have demonstrated the efficacy of FF:polymer nanocomposites by a selfassembly process. Although the overall EL efficiency of FF:PF devices is lower than pristine polyfluorene LEDs, improved strategies in aligning the FF nanotubes and a better polymer:FF interaction may mitigate some of these bottlenecks in the future. This work, for the first time, demonstrates that self-assembled FF nanotubes with conjugated polymers have potential application in organic electronics. PF8 with its long di-octyl side chains shows a better binding affinity toward FF-MNS compared to PF2/6. MD simulations show a higher van der Waals interaction energy for FF:PF8 compared with FF:PF2/6, corroborating the optical spectroscopy and electron microscopy results. Enzyme mediated biodegradability tests were performed from FF:PF8 films and compared with FF-MNS and PF8. The FF-MNS film is almost fully biodegradable within a couple of hours. FF:PF8 is $\approx 85 \%$ biodegradable compared to pristine $\mathrm{PF} 8$, which does not undergo any enzymemediated biodegradation. Integration of peptide nanostructures with conducting polymers 
opens up a new generation of biodegradable hybrid materials for application in organic electronics. 


\section{$7 \quad$ Summary and Future Work}

This work contributes to the basic understanding of FF based peptide nanostructures for electronic, optical, and magnetic applications. Attractive properties including piezoelectricity, thermal and chemical stability, and relatively simple functionalization make peptide nanostructures potential candidates for several applications in drug delivery, biosensors, waveguides, scaffolding, and light emitting displays. This thesis performs detailed investigation of magnetic, nonlinear optical, and electronic properties of FF-based peptide nanostructures as well as some photonic, magnetic, and organic electronic devices using FF based peptide nanostructures, and a summary of all these research works is shown in a schematic presentation (Figure 7.1).

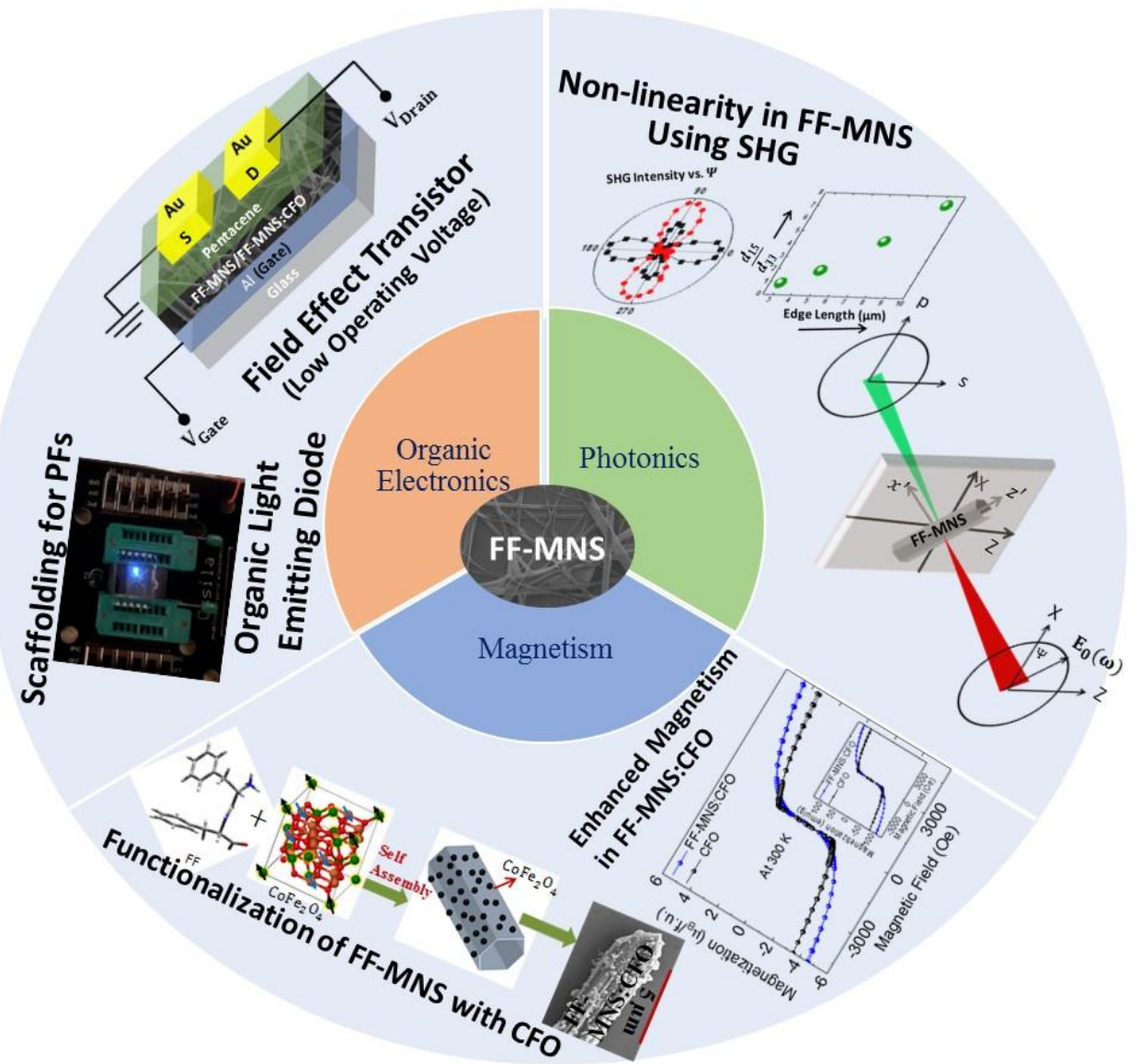

Figure 7.1. Schematic presentation of the summary for this thesis. 


\section{Photonic Application}

Nonlinear optics is an essential component of modern laser systems and optoelectronic devices. ${ }^{137}$ We probed the non-linear properties of FF-MNs using SHG. The relationship between nonlinearity and structure of peptide tubes has been achieved. SHG polarimetry from individual self-assembled peptide micro/nanotubes allows a precise measurement of the symmetry of the tubes. One of the transverse nonlinear components $d_{31}$ is found to be negative. The ratio of the transverse to the longitudinal nonlinear components $d_{15} / d_{33}$ and $\left|d_{31} / d_{33}\right|$ increase with the diameter of the tubes, shedding light for the first time on the anisotropy of the nonlinear coefficients in FF-MNTs. SHG polarimetry from FF-MNTs annealed at different annealing temperatures show an enhancement of the nonlinear coefficients $\left(d_{15} / d_{33}\right.$ and $\left.\left|d_{31} / d_{33}\right|\right)$, suggesting a higher alignment of the ferroelectric domains upon increasing annealing temperatures. The increase in the nonlinear coefficients with the microtube diameter in SHG is related to the number of unit cells per unit volume and the changes in the SHG coefficients with annealing temperatures reflect a slight shrinking of the tube diameter followed by a re-arrangement of ferroelectric domains, enhancing the dipole moment of the system. SHG polarimetry from bio-organic nanostructures proves to be a powerful tool for obtaining structure-property relationships. Furthermore, the biocompatible and rigid nature of peptide micro/nanostructures open up a sensitive tool for integrating nanophotonics with biosensing activities.

\section{Magnetic Application}

Amongst magnetic nanoparticles, cobalt ferrite $\left(\mathrm{CoFe}_{2} \mathrm{O}_{4}\right.$ or $\left.\mathrm{CFO}\right)$ has received a lot of interest in biomedical applications. ${ }^{81}$ This is predominantly due to its moderate values of saturation magnetization, high magneto-crystalline anisotropy, high Curie temperature, 
and easy tunability of particle size ${ }^{82}$, facilitating possible use as a contrast agent for magnetic resonance imaging and targeted drug delivery. Functionalized FF-MNS with CFO may be used for these applications. These require an understanding of the interaction mechanism of FF-MNS with CFO. A combination of structural and spectroscopic investigations confirms the interaction mechanism of FF-MNS with CFO nanoparticles. The inverse spinel structure of $\mathrm{CFO}$ with $\mathrm{Fe}$ and $\mathrm{Co}$ ions both in the $\mathrm{O}_{\mathrm{h}}$ and $\mathrm{T}_{\mathrm{d}}$ site provides interaction sites with the amine group in FF-MNS, where either the Co ions or both Fe and Co ions give up an electron to the amine group. In addition, functionalization of bioinspired peptide nanostructures FF-MNS with magnetic nanoparticles $\mathrm{CFO}$ provides an attractive path towards enhancing the magnetic properties.

\section{Organic Electronics}

FF-MNS have been used as a template for PFs which open up a new route toward bottom-up scalable processes for organic electronics. Owing to the nanostructuring effect of FF peptides, the surface area of the active polymer is greatly enhanced in FF:PF nanocomposites. This is economical in electronic devices since a small wt $\%$ of the polymer can result in similar device performance compared to only conjugated polymer films as the active layer. Characterization studies such as SEM, TEM, PL, EL, and MD simulation confirm the better binding of PF8 compared to PF2/6 to FF-MNS. Being $80 \%$ biodegradable, FF-MNS:PFs composites may be used as an active layer in biodegradable OLED device. Further, the dielectric properties of FF-MNS and FF-MNS:CFO were exploited in pentacene MIS (Metal-Insulator-Semiconductor) capacitors and FETs. A higher value of capacitance in FF-MNS:CFO compared to FF-MNS, observed in MIS 
capacitors, is responsible for lowering the operating voltages in pentacene FETs. These results have ramifications in low operating voltage floating gate memory FETs.

\section{Future Work}

Our research opens up new avenues for future research. These include:

\section{(1) Multiferroic Application}

A Multiferoic material has both magnetism and ferroelectricity at the same time, and it has potential for applications as actuators, switches, magnetic field sensors or new types of electronic memory devices. The magneto-electric effect facilitates the conversion between energies stored in magnetic and electric fields. In electro-magnetic multiferroic, the electric polarization is modified with applied external magnetic field; conversely, the application of an external electric field alters the net magnetization. Magneto-electric coupling coefficient, $\alpha$ is used to quantify the electro-magneto effect in multiferroic. Experimentally $\alpha$ is determined in multiferroic materials. The electrically induced magnetoelectric effect is defined as the change in the magnetization (M) of the sample due to the application of an electric field $(\mathrm{E})$ :

$$
\alpha_{E}=\frac{\partial M}{\partial E}
$$

Similarly, the magnetically induced magneto-electric effect is the change in the electric polarization $(\mathrm{P})$ of the sample due to the application of a magnetic field $(\mathrm{H})$ :

$$
\alpha_{H}=\frac{\partial P}{\partial H} \text {. }
$$

We have seen (Chapter 5) that field effect transistor, based on peptide nanostructures functionalized with cobalt ferrite as dielectric, reduces the operating voltage. Figure 7.2 shows our preliminary result for magnetic field dependent capacitance in FF-MNS:CFO. 
Increased capacitance is observed in FF-MNS:CFO dielectric with applied magnetic field of $0.2 \mathrm{~T}$. This indicates that there is a possibility of existing multiferoic property in the peptide and cobalt ferrite composite material. For multiferoic application, further studies like magnetic field dependent ferroelectricity and electric field dependent magnetism in FF-MNS:CFO may be explored.

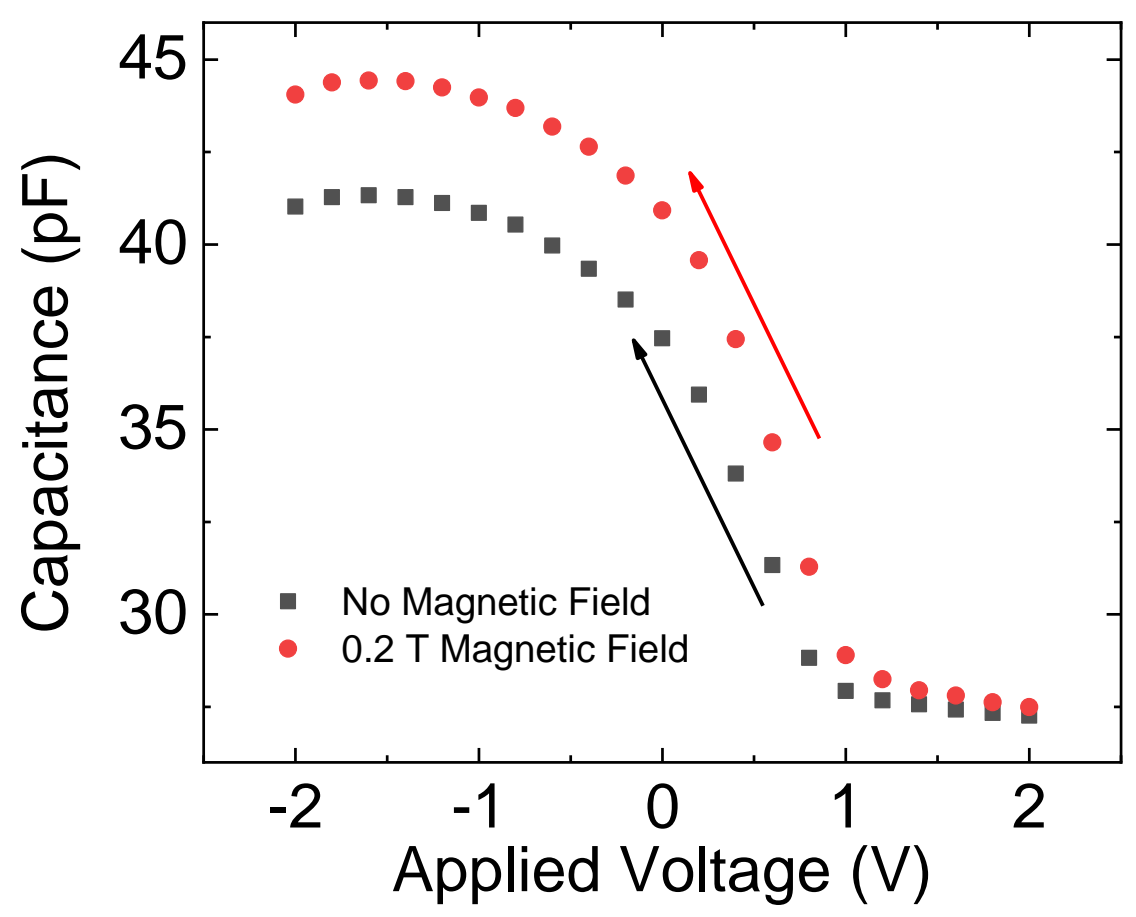

Figure 7.2. Capacitance versus voltage from a pentacene MIS capacitor with no magnetic field (black square) and in the presence of $0.2 \mathrm{~T}$ magnetic field (red circles). Both $\mathrm{C}-\mathrm{V}$ curves were swept from positive to negative voltages.

\section{(2) Fluorinated FF (F-FF) nanostructures for enhanced SHG and piezoelectricity}

As mentioned previously (Chapter 3), FF is a zwitterionic molecule where positive charge is on NH3 group and negative charge is on - $\mathrm{COOH}$ group, shown in Figure 7.2 (a). The dipole moment in FF molecule arises because of these separated charges. Fluorine which is highly electronegative element can enhance the hyperpolarizability if it is introduced in the structure. Two hydrogen atoms attached with the phenyl rings are 
replaced with two fluorine atoms in F-FF molecule. This replacement would not disturb the self-assembly process much.

(a)

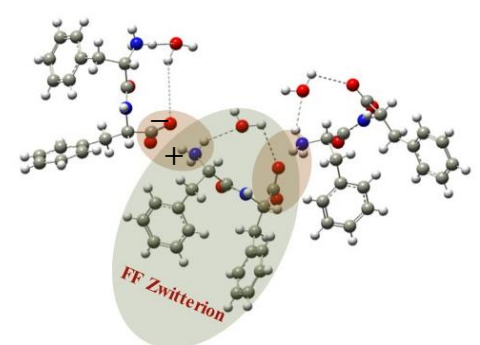

(b)

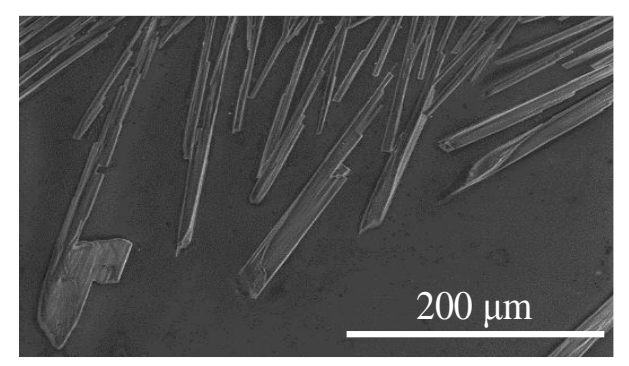

Figure7.3. (a) Three FF units in FF-MNS crystal.(b) SEM image of Fluorinated FF nanostructures.

Figure 7.3 (b) shows the SEM image of F-FF nanostructures. As it is clear from the SEM images that F-FF forms well defined crystal structures. Structural analysis of these nanostructures using Rietveld analysis and High-resolution TEM imaging can be accomplished to determine the crystal structure. Future studies of these F-FF based nanostructures will benefit in enhancing SHG response. 


\section{REFERENCES}

1. Adler-Abramovich, L.; Gazit, E., The physical properties of supramolecular peptide assemblies: from building block association to technological applications. Chemical Society Reviews 2014, 43 (20), 6881-6893.

2. Reches, M.; Gazit, E., Molecular self-assembly of peptide nanostructures: mechanism of association and potential uses. Current Nanoscience 2006, 2, 105-111.

3. $\quad$ Liberato, M. S.; Kogikoski, S., Jr.; Silva, E. R.; Coutinho-Neto, M. D.; Scott, L. P.; Silva, R. H.; Oliveira, V. X., Jr.; Ando, R. A.; Alves, W. A., Self-assembly of Arg-Phe nanostructures via the solid-vapor phase method. The journal of physical chemistry. B 2013, 117 (3), 733-40.

4. $\quad$ Souza, M. I.; Jaques, Y. M.; de Andrade, G. P.; Ribeiro, A. O.; da Silva, E. R.; Fileti, E. E.; Avilla Ede, S.; Pinheiro, M. V.; Krambrock, K.; Alves, W. A., Structural and photophysical properties of peptide micro/nanotubes functionalized with hypericin. The journal of physical chemistry. B 2013, 117 (9), 2605-14.

5. Adler-Abramovich, L.; Aronov, D.; Beker, P.; Yevnin, M.; Stempler, S.; Buzhansky, L.; Rosenman, G.; Gazit, E., Self-assembled arrays of peptide nanotubes by vapour deposition. Nature Nanotechnology 2009, 4, 849.

6. $\quad$ Singh, G.; Bittner, A. M.; Loscher, S.; Malinowski, N.; Kern, K., Electrospinning of Diphenylalanine Nanotubes. Advanced Materials 2008, 20 (12), 2332-2336.

7. Cipriano, T.; Knotts, G.; Laudari, A.; Bianchi, R. C.; Alves, W. A.; Guha, S., Bioinspired Peptide Nanostructures for Organic Field-Effect Transistors. ACS Appl. Mater. Interfaces 2014, 6 (23), 21408-21415.

8. $\quad$ Bianchi, R. C.; da Silva, E. R.; Dall'Antonia, L. H.; Ferreira, F. F.; Alves, W. A., A nonenzymatic biosensor based on gold electrodes modified with peptide self-assemblies for detecting ammonia and urea oxidation. Langmuir : the ACS journal of surfaces and colloids 2014, 30 (38), 11464-73.

9. Cullity, B. D., Elements of X-ray Diffraction Addison Wesley 1978, 2 nd Edition.

10. Birkholz, M., Thin Film Analysis by X-Ray Scattering. John Wiley \& Sons 2006.

11. John F. Watts, J. W., An Introduction to Surface Analysis by XPS and AES. Wiley 2003.

12. Zhou, W.; Apkarian, R.; Wang, Z. L.; Joy, D., Fundamentals of Scanning Electron Microscopy (SEM). In Scanning Microscopy for Nanotechnology: Techniques and Applications, Zhou, W.; Wang, Z. L., Eds. Springer New York: New York, NY, 2007; pp $1-40$.

13. Long, D. A., The Raman Effect: A Unified Treatment of the Theory of Raman Scattering by Molecules. Wiley 2002.

14. Matti, K.; Suchismita, G., Blue emitting organic semiconductors under high pressure: status and outlook. Reports on Progress in Physics 2016, 79 (6), 066601.

15. Singha, A.; Dhar, P.; Roy, A., A nondestructive tool for nanomaterials: Raman and photoluminescence spectroscopy. American Journal of Physics 2005, 73 (3), 224-233.

16. Denev, S. A.; Lummen, T. T. A.; Barnes, E.; Kumar, A.; Gopalan, V., Probing Ferroelectrics Using Optical Second Harmonic Generation. Journal of the American Ceramic Society 2011, 94 (9), 2699-2727.

17. Görbitz, C. H., Microporous Organic Materials from Hydrophobic Dipeptides. Chemistry - A European Journal 2007, 13 (4), 1022-1031. 
18. Kholkin, A.; Amdursky, N.; Bdikin, I.; Gazit, E.; Rosenman, G., Strong Piezoelectricity in Bioinspired Peptide Nanotubes. ACS Nano 2010, 4 (2), 610-614.

19. Handelman, A.; Lavrov, S.; Kudryavtsev, A.; Khatchatouriants, A.; Rosenberg, Y.; Mishina, E.; Rosenman, G., Nonlinear Optical Bioinspired Peptide Nanostructures. Advanced Optical Materials 2013, 1 (11), 875-884.

20. Gramm, K.; Lundgren, L.; Beckman, O., SQUID Magnetometer for Mangetization Measurements. Physica Scripta 1976, 13 (2), 93.

21. http://www.youtube.com/watch?v=epdx9d4D_YQ.

22. M. J. Frisch, G. W. T., H. B. Schlegel, G. E. Scuseria, M. A. Robb, J. R. Cheeseman, G. Scalmani, V. Barone, B. Mennucci, G. A. Petersson, H. Nakatsuji, M. Caricato, X. Li, H. P. Hratchian, A. F. Izmaylov, J. Bloino, G. Zheng, J. L. Sonnenberg, M. Hada, M. Ehara, K. Toyota, R. Fukuda, J. Hasegawa, M. Ishida, T. Nakajima, Y. Honda, O. Kitao, H. Nakai, T. Vreven, J. A. Montgomery, Jr., J. E. Peralta, F. Ogliaro, M. Bearpark, J. J. Heyd, E. Brothers, K. N. Kudin, V. N. Staroverov, R. Kobayashi, J. Normand, K. Raghavachari, A. Rendell, J. C. Burant, S. S. Iyengar, J. Tomasi, M. Cossi, N. Rega, J. M. Millam, M. Klene, J. E. Knox, J. B. Cross, V. Bakken, C. Adamo, J. Jaramillo, R. Gomperts, R. E. Stratmann, O. Yazyev, A. J. Austin, R. Cammi, C. Pomelli, J. W. Ochterski, R. L. Martin, K. Morokuma, V. G. Zakrzewski, G. A. Voth, P. Salvador, J. J. Dannenberg, S. Dapprich, A. D. Daniels, Ö. Farkas, J. B. Foresman, J. V. Ortiz, J. Cioslowski and D. J. Fox, Gaussian 09 ,Revision E.01, Gaussian 2009, Inc., Wallingford CT, .

23. Hay, P. J.; Wadt, W. R., Ab initio effective core potentials for molecular calculations. Potentials for the transition metal atoms Sc to $\mathrm{Hg}$. The Journal of Chemical Physics 1985, 82 (1), 270-283.

24. Volz, C.; Arif, M.; Guha, S., Conformations in dioctyl substituted polyfluorene: A combined theoretical and experimental Raman scattering study. The Journal of Chemical Physics 2007, 126 (6), 064905.

25. Adil, D.; Guha, S., Surface-enhanced Raman spectroscopic studies of the Aupentacene interface: A combined experimental and theoretical investigation. The Journal of Chemical Physics 2013, 139 (4), 044715.

26. Wang, J.; Liu, K.; Xing, R.; Yan, X., Peptide self-assembly: thermodynamics and kinetics. Chemical Society reviews 2016, 45 (20), 5589-5604.

27. Huang, R.; Qi, W.; Su, R.; Zhao, J.; He, Z., Solvent and surface controlled selfassembly of diphenylalanine peptide: from microtubes to nanofibers. Soft Matter 2011, 7 (14), 6418-6421.

28. Wang, J.; Liu, K.; Yan, L.; Wang, A.; Bai, S.; Yan, X., Trace Solvent as a Predominant Factor To Tune Dipeptide Self-Assembly. ACS Nano 2016, 10 (2), 21382143.

29. Andrade-Filho, T.; Martins, T. C.; Ferreira, F. F.; Alves, W. A.; Rocha, A. R., Water-driven stabilization of diphenylalanine nanotube structures. Theoretical Chemistry Accounts 2016, 135 (8), 185.

30. Heredia, A.; Bdikin, I.; Kopyl, S.; Mishina, E.; Semin, S.; Sigov, A.; German, K.; Bystrov, V.; Gracio, J.; Kholkin, A. L., Temperature-driven phase transformation in selfassembled diphenylalanine peptide nanotubes. Journal of Physics D: Applied Physics 2010, 43 (46), 462001. 
31. Bdikin, I.; Bystrov, V.; Kopyl, S.; Lopes, R. P. G.; Delgadillo, I.; Gracio, J.; Mishina, E.; Sigov, A.; Kholkin, A. L., Evidence of ferroelectricity and phase transition in pressed diphenylalanine peptide nanotubes. Applied Physics Letters 2012, 100 (4), 043702. 32. Bystrov, V. S.; Seyedhosseini, E.; Kopyl, S.; Bdikin, I. K.; Kholkin, A. L., Piezoelectricity and ferroelectricity in biomaterials: Molecular modeling and piezoresponse force microscopy measurements. Journal of Applied Physics 2014, 116 (6), 066803.

33. Amdursky, N.; Beker, P.; Koren, I.; Bank-Srour, B.; Mishina, E.; Semin, S.; Rasing, T.; Rosenberg, Y.; Barkay, Z.; Gazit, E.; Rosenman, G., Structural Transition in Peptide Nanotubes. Biomacromolecules 2011, 12, 1349-1354.

34. Ren, M.-L.; Agarwal, R.; Liu, W.; Agarwal, R., Crystallographic Characterization of II-VI Semiconducting Nanostructures via Optical Second Harmonic Generation. Nano Letters 2015, 15 (11), 7341-7346.

35. Grinblat, G.; Rahmani, M.; Cortés, E.; Caldarola, M.; Comedi, D.; Maier, S. A.; Bragas, A. V., High-Efficiency Second Harmonic Generation from a Single Hybrid ZnO Nanowire/Au Plasmonic Nano-Oligomer. Nano Letters 2014, 14 (11), 6660-6665.

36. Pecora, E. F.; Walsh, G. F.; Forestiere, C.; Handin, A.; Russo-Averchi, E.; DalmauMallorqui, A.; Canales-Mundet, I.; Fontcuberta i Morral, A.; Negro, L. D., Enhanced second harmonic generation from InAs nano-wing structures on silicon. Nanoscale 2013, 5 (21), 10163-10170.

37. Kumar, N.; Najmaei, S.; Cui, Q.; Ceballos, F.; Ajayan, P. M.; Lou, J.; Zhao, H., Second harmonic microscopy of monolayer MoS2. Physical Review B 2013, 87 (16), 161403.

38. Shen, Y. R., The principles of nonlinear optics. Wiley: New York, 1984.

39. Zhang, H.; Liao, Q.; Wang, X.; Xu, Z.; Fu, H., Self-assembled organic hexagonal micro-prisms with high second harmonic generation efficiency for photonic devices. Nanoscale 2015, 7 (22), 10186-10192.

40. Bianchi, R. C.; da Silva, E. R.; Dall‘Antonia, L. H.; Ferreira, F. F.; Alves, W. A., A Nonenzymatic Biosensor Based on Gold Electrodes Modified with Peptide SelfAssemblies for Detecting Ammonia and Urea Oxidation. Langmuir : the ACS journal of surfaces and colloids 2014, 30 (38), 11464-11473.

41. Lekprasert, B.; Korolkov, V.; Falamas, A.; Chis, V.; Roberts, C. J.; Tendler, S. J. B.; Notingher, I., Investigations of the Supramolecular Structure of Individual Diphenylalanine Nano- and Microtubes by Polarized Raman Microspectroscopy. Biomacromolecules 2012, 13 (7), 2181-2187.

42. Wu, X.; Xiong, S.; Wang, M.; Shen, J.; Chu, P. K., Water-Sensitive HighFrequency Molecular Vibrations in Self-Assembled Diphenylalanine Nanotubes. The Journal of Physical Chemistry C 2012, 116 (17), 9793-9799.

43. Wu, X.; Xiong, S.; Wang, M.; Shen, J.; Chu, P. K., Low-frequency Raman scattering of bioinspired self-assembled diphenylalanine nanotubes/microtubes. Opt. Express 2012, 20 (5), 5119-5126.

44. Pankhurst, Q. A.; Connolly, J.; Jones, S. K.; Dobson, J., Applications of magnetic nanoparticles in biomedicine. Journal of Physics D: Applied Physics 2003, 36 (13), R167. 45. Xu, M.; Li, J.; Iwai, H.; Mei, Q.; Fujita, D.; Su, H.; Chen, H.; Hanagata, N., Formation of Nano-Bio-Complex as Nanomaterials Dispersed in a Biological Solution for Understanding Nanobiological Interactions. Scientific Reports 2012, 2, 406. 
46. Nel, A. E.; Madler, L.; Velegol, D.; Xia, T.; Hoek, E. M. V.; Somasundaran, P.; Klaessig, F.; Castranova, V.; Thompson, M., Understanding biophysicochemical interactions at the nano-bio interface. Nat Mater 2009, 8 (7), 543-557.

47. Bhaumik, A.; Shearin, A. M.; Delong, R.; Wanekaya, A.; Ghosh, K., Probing the Interaction at the Nano-Bio Interface Using Raman Spectroscopy: ZnO Nanoparticles and Adenosine Triphosphate Biomolecules. Journal of Physical Chemistry C 2014, 118 (32), 18631-18639.

48. Vertegel, A. A.; Siegel, R. W.; Dordick, J. S., Silica Nanoparticle Size Influences the Structure and Enzymatic Activity of Adsorbed Lysozyme. Langmuir 2004, 20 (16), 6800-6807.

49. Shemetov, A. A.; Nabiev, I.; Sukhanova, A., Molecular Interaction of Proteins and Peptides with Nanoparticles. ACS Nano 2012, 6 (6), 4585-4602.

50. Khanra, S.; Cipriano, T.; Lam, T.; White, T. A.; Fileti, E. E.; Alves, W. A.; Guha, S., Self-Assembled Peptide-Polyfluorene Nanocomposites for Biodegradable Organic Electronics. Advanced Materials Interfaces 2015, 2, 1500265.

51. Schlücker, S., Surface-Enhanced Raman Spectroscopy: Concepts and Chemical Applications. Angewandte Chemie International Edition 2014, 53 (19), 4756-4795.

52. Muehlethaler, C.; Leona, M.; Lombardi, J. R., Review of Surface Enhanced Raman Scattering Applications in Forensic Science. Analytical Chemistry 2016, 88 (1), 152-169.

53. Yuan, Y.; Panwar, N.; Yap, S. H. K.; Wu, Q.; Zeng, S.; Xu, J.; Tjin, S. C.; Song, J.; Qu, J.; Yong, K.-T., SERS-based ultrasensitive sensing platform: An insight into design and practical applications. Coordination Chemistry Reviews 2017, 337, 1-33.

54. Huang, J.-A.; Zhang, Y.-L.; Ding, H.; Sun, H.-B., SERS-Enabled Lab-on-a-Chip Systems. Advanced Optical Materials 2015, 3 (5), 618-633.

55. Fateixa, S.; Nogueira, H. I. S.; Trindade, T., Hybrid nanostructures for SERS: materials development and chemical detection. Physical Chemistry Chemical Physics 2015, 17 (33), 21046-21071.

56. Ross, M. B.; Ashley, M. J.; Schmucker, A. L.; Singamaneni, S.; Naik, R. R.; Schatz, G. C.; Mirkin, C. A., Structure-Function Relationships for Surface-Enhanced Raman Spectroscopy-Active Plasmonic Paper. The Journal of Physical Chemistry C 2016, 120 (37), 20789-20797.

57. Si, K. J.; Sikdar, D.; Chen, Y.; Eftekhari, F.; Xu, Z.; Tang, Y.; Xiong, W.; Guo, P.; Zhang, S.; Lu, Y.; Bao, Q.; Zhu, W.; Premaratne, M.; Cheng, W., Giant Plasmene Nanosheets, Nanoribbons, and Origami. ACS Nano 2014, 8 (11), 11086-11093.

58. Zhang, N.; Liu, K.; Liu, Z.; Song, H.; Zeng, X.; Ji, D.; Cheney, A.; Jiang, S.; Gan, Q., Ultrabroadband Metasurface for Efficient Light Trapping and Localization: A Universal Surface-Enhanced Raman Spectroscopy Substrate for "All" Excitation Wavelengths. Advanced Materials Interfaces 2015, 2 (10), 1500142.

59. Basuray, S.; Pathak, A.; Bok, S.; Chen, B.; Hamm, S., C. ; Mathai, C., J.; Guha, S.; Gangopadhyay, K.; Gangopadhyay, S., Plasmonic nano-protrusions: hierarchical nanostructures for single-molecule Raman spectroscopy. Nanotechnology 2017, 28 (2), 025302.

60. Almohammed, S.; Oladapo, S. O.; Ryan, K.; Kholkin, A. L.; Rice, J. H.; Rodriguez, B. J., Wettability gradient-induced alignment of peptide nanotubes as templates for biosensing applications. RSC Advances 2016, 6 (48), 41809-41815. 
61. Castillo, J. J.; Rindzevicius, T.; Wu, K.; Schmidt, M. S.; Janik, K. A.; Boisen, A.; Svendsen, W.; Rozlosnik, N.; Castillo-León, J., Synthesis and characterization of covalent diphenylalanine nanotube-folic acid conjugates. Journal of Nanoparticle Research 2014, 16 (7), 2525.

62. Su, Y.; He, Q.; Yan, X.; Fei, J.; Cui, Y.; Li, J., Peptide Mesocrystals as Templates to Create an Au Surface with Stronger Surface-Enhanced Raman Spectroscopic Properties. Chemistry - A European Journal 2011, 17 (12), 3370-3375.

63. Reches, M.; Gazit, E., Casting Metal Nanowires Within Discrete Self-Assembled Peptide Nanotubes. Science 2003, 300 (5619), 625-627.

64. Heredia, A.; Bdikin, I.; Kopyl, S.; Mishina, E.; Semin, S.; Sigov, A.; German, K.; Bystrov, V.; Gracio, J.; Kholkin, A. L., Temperature-driven phase transformation in selfassembled diphenylalanine peptide nanotubes. J. Phys. D: Appl. Phys. 2010, 43 (46).

65. Ryu, J.; Park, C. B., High stability of self-assembled peptide nanowires against thermal, chemical, and proteolytic attacks. Biotechnology and Bioengineering 2010, 105 (2), 221-230.

66. Kogikoski, S.; Sousa, C. P.; Liberato, M. S.; Andrade-Filho, T.; Prieto, T.; Ferreira, F. F.; Rocha, A. R.; Guha, S.; Alves, W. A., Multifunctional biosensors based on peptidepolyelectrolyte conjugates. Physical Chemistry Chemical Physics 2016, 18 (4), 3223-3233. 67. Lin, S.; Li, M.; Dujardin, E.; Girard, C.; Mann, S., One-Dimensional Plasmon Coupling by Facile Self-Assembly of Gold Nanoparticles into Branched Chain Networks. Advanced Materials 2005, 17 (21), 2553-2559.

68. Li, H.; Xia, H.; Wang, D.; Tao, X., Simple Synthesis of Monodisperse, Quasispherical, Citrate-Stabilized Silver Nanocrystals in Water. Langmuir : the ACS journal of surfaces and colloids 2013, 29 (16), 5074-5079.

69. Amaral, H. R.; Kogikoski Jr, S.; Silva, E. R.; Souza, J. A.; Alves, W. A., Microand nano-sized peptidic assemblies prepared via solid-vapor approach: Morphological and spectroscopic aspects. Materials Chemistry and Physics 2012.

70. Lee, J. S.; Yoon, I.; Kim, J.; Ihee, H.; Kim, B.; Park, C. B., Self-Assembly of Semiconducting Photoluminescent Peptide Nanowires in the Vapor Phase. Angew Chem Int Ed Engl 2010.

71. Semin, S.; van Etteger, A.; Cattaneo, L.; Amdursky, N.; Kulyuk, L.; Lavrov, S.; Sigov, A.; Mishina, E.; Rosenman, G.; Rasing, T., Strong Thermo-Induced Single And Two-Photon Green Luminescence In Self-Organized Peptide Microtubes. Small (Weinheim an der Bergstrasse, Germany) 2015, 11 (9-10), 1156-1160.

72. Zhang, J.; Li, X.; Sun, X.; Li, Y., Surface Enhanced Raman Scattering Effects of Silver Colloids with Different Shapes. The Journal of Physical Chemistry B 2005, 109 (25), 12544-12548.

73. Watanabe, H.; Hayazawa, N.; Inouye, Y.; Kawata, S., DFT vibrational calculations of rhodamine 6G adsorbed on silver: analysis of tip-enhanced Raman spectroscopy. The Journal of Physical Chemistry B 2005, 109 (11), 5012-5020.

74. Watanabe, H.; Hayazawa, N.; Inouye, Y.; Kawata, S., DFT Vibrational Calculations of Rhodamine 6G Adsorbed on Silver: Analysis of Tip-Enhanced Raman Spectroscopy. The Journal of Physical Chemistry B 2005, 109 (11), 5012-5020.

75. A. N. Subbotina, G. M. Zhidomirova, b, I. R. Subbotinaa, and V. B. Kazanskya, Molecular and Dissociative Adsorption of H2O on Zeolite Zn/ZSM_5 Studied by 
Diffuse_Reflectance IR Spectroscopy and Quantum Chemical Calculations,. Kinetics and Catalysis 2013, 54, 744-748.

76. Cyriac, J.; Li, G.; Cooks, R. G., Vibrational spectroscopy and mass spectrometry for characterization of soft landed polyatomic molecules. Analytical chemistry 2011, 83 (13), 5114-21.

77. He, X. N.; Gao, Y.; Mahjouri-Samani, M.; Black, P. N.; Allen, J.; Mitchell, M.; Xiong, W.; Zhou, Y. S.; Jiang, L.; Lu, Y. F., Surface-enhanced Raman spectroscopy using gold-coated horizontally aligned carbon nanotubes. Nanotechnology 2012, 23 (20), 205702.

78. Fan, M.; Andrade, G. F. S.; Brolo, A. G., A review on the fabrication of substrates for surface enhanced Raman spectroscopy and their applications in analytical chemistry. Analytica Chimica Acta 2011, 693 (1-2), 7-25.

79. Škantárová, L.; Oriňák, A.; Oriňáková, R.; Jerigová, M.; Stupavská, M.; Velič, D., Functional silver nanostructured surfaces applied in SERS and SIMS. Surface and Interface Analysis 2013, 45 (8), 1266-1272.

80. Guha, S.; Menéndez, J.; Page, J. B.; Adams, G. B., Empirical bond polarizability model for fullerenes. Physical Review B 1996, 53 (19), 13106-13114.

81. Jun, Y.-w.; Seo, J.-w.; Cheon, J., Nanoscaling Laws of Magnetic Nanoparticles and Their Applicabilities in Biomedical Sciences. Accounts of Chemical Research 2008, 41 (2), 179-189.

82. Maaz, K.; Mumtaz, A.; Hasanain, S. K.; Ceylan, A., Synthesis and magnetic properties of cobalt ferrite $(\mathrm{CoFe} 2 \mathrm{O} 4)$ nanoparticles prepared by wet chemical route. Journal of Magnetism and Magnetic Materials 2007, 308 (2), 289-295.

83. Sanpo, N.; Berndt, C. C.; Wen, C.; Wang, J., Transition metal-substituted cobalt ferrite nanoparticles for biomedical applications. Acta Biomaterialia 2013, 9 (3), 58305837.

84. Amiri, S.; Shokrollahi, H., The role of cobalt ferrite magnetic nanoparticles in medical science. Materials Science and Engineering: C 2013, 33 (1), 1-8.

85. Jung, J. H.; Kim, S.; Kim, H.; Park, J.; Oh, J. H., High-Performance Flexible Organic Nano-Floating Gate Memory Devices Functionalized with Cobalt Ferrite Nanoparticles. Small 2015, 11 (37), 4976-4984.

86. Liberato, M. S.; Kogikoski, S.; da Silva, E. R.; de Araujo, D. R.; Guha, S.; Alves, W. A., Polycaprolactone fibers with self-assembled peptide micro/nanotubes: a practical route towards enhanced mechanical strength and drug delivery applications. Journal of Materials Chemistry B 2016, 4 (8), 1405-1413.

87. Silva, R. F.; Araújo, D. R.; Silva, E. R.; Ando, R. A.; Alves, W. A., 1Diphenylalanine Microtubes As a Potential Drug-Delivery System: Characterization, Release Kinetics, and Cytotoxicity. Langmuir 2013, 29 (32), 10205-10212.

88. Ryu, J.; Kim, S.-W.; Kang, K.; Park, C. B., Synthesis of Diphenylalanine/Cobalt Oxide Hybrid Nanowires and Their Application to Energy Storage. ACS Nano 2010, 4 (1), 159-164.

89. Ferreira, F. F.; Bueno, P. R.; Setti, G. O.; Giménez-Romero, D.; García-Jareño, J. J.; Vicente, F., Resonant X-ray diffraction as a tool to calculate mixed valence ratios: Application to Prussian Blue materials. Appl. Phys. Lett. 2008, 92 (26), 264103.

90. Rietveld, H. M., Line profiles of neutron powder-diffraction peaks for structure refinement. Acta Crystallogr. 1967, 22 (1), 151-152. 
91. Rietveld, H. M., A Profile Refinement Method for Nuclear and Magnetic Structures. J. Appl. Crystallogr. 1969, 2 (2), 65-71.

92. Toby, B. H., R factors in Rietveld analysis: How good is good enough? Powder Diffr. 2006, 21 (1), 67-70.

93. Hill, R. J.; Howard, C. J., Quantitative phase analysis from neutron powder diffraction data using the Rietveld method. J. Appl. Crystallogr. 1987, 20 (6), 467-474.

94. Sun, H.; Yang, X.; Zhao, L.; Xu, T.; Lian, J., One-pot hydrothermal synthesis of octahedral $\mathrm{CoFe} / \mathrm{CoFe} 2 \mathrm{O} 4$ submicron composite as heterogeneous catalysts with enhanced peroxymonosulfate activity. Journal of Materials Chemistry A 2016, 4 (24), 9455-9465.

95. Gu, Z.; Xiang, X.; Fan, G.; Li, F., Facile Synthesis and Characterization of Cobalt Ferrite Nanocrystals via a Simple Reduction-Oxidation Route. The Journal of Physical Chemistry C 2008, 112 (47), 18459-18466.

96. Pradhan, D. K.; Kumari, S.; Puli, V. S.; Das, P. T.; Pradhan, D. K.; Kumar, A.; Scott, J. F.; Katiyar, R. S., Correlation of dielectric, electrical and magnetic properties near the magnetic phase transition temperature of cobalt zinc ferrite. Physical Chemistry Chemical Physics 2017, 19 (1), 210-218.

97. Egelhoff, W. F., Core-level binding-energy shifts at surfaces and in solids. Surface Science Reports 1987, 6 (6), 253-415.

98. Hosterman, B. D.; Farley, J. W.; Johnson, A. L., Spectroscopic study of the vibrational modes of magnesium nickel chromite. Journal of Physics and Chemistry of Solids 2013, 74 (7), 985-990.

99. Rygula, A.; Majzner, K.; Marzec, K. M.; Kaczor, A.; Pilarczyk, M.; Baranska, M., Raman spectroscopy of proteins: a review. Journal of Raman Spectroscopy 2013, 44 (8), 1061-1076.

100. El-Gendy, A. A.; Bertino, M.; Clifford, D.; Qian, M.; Khanna, S. N.; Carpenter, E. E., Experimental evidence for the formation of $\mathrm{CoFe} 2 \mathrm{C}$ phase with colossal magnetocrystalline-anisotropy. Appl. Phys. Lett. 2015, 106 (21), 213109.

101. Seehra, M. S.; Babu, V. S.; Manivannan, A.; Lynn, J. W., Neutron scattering and magnetic studies of ferrihydrite nanoparticles. Phys. Rev. B 2000, 61 (5), 3513-3518.

102. Makhlouf, S. A.; Parker, F. T.; Berkowitz, A. E., Magnetic hysteresis anomalies in ferritin. Phys. Rev. B 1997, 55 (22), R14717-R14720.

103. Tiwari, S. D.; Rajeev, K. P., Effect of distributed particle magnetic moments on the magnetization of $\mathrm{NiO}$ nanoparticles. Solid State Communications 2012, 152 (12), 10801083.

104. Dutta, P.; Manivannan, A.; Seehra, M. S.; Shah, N.; Huffman, G. P., Magnetic properties of nearly defect-free maghemite nanocrystals. Phys. Rev. B 2004, 70 (17), 174428.

105. Elmore, W. C., The Magnetization of Ferromagnetic Colloids. Phys. Rev. 1938, 54 (12), 1092-1095.

106. Ramana, C. V.; Kolekar, Y. D.; Bharathi, K. K.; Sinha, B.; Ghosh, K., Correlation between structural, magnetic, and dielectric properties of manganese substituted cobalt ferrite. J. Appl. Phys. 2013, 114 (18), 183907.

107. Paulsen, J. A.; Ring, A. P.; Lo, C. C. H.; Snyder, J. E.; Jiles, D. C., Manganesesubstituted cobalt ferrite magnetostrictive materials for magnetic stress sensor applications. J. Appl. Phys. 2005, 97 (4), 044502. 
108. Melikhov, Y.; Snyder, J. E.; Jiles, D. C.; Ring, A. P.; Paulsen, J. A.; Lo, C. C. H.; Dennis, K. W., Temperature dependence of magnetic anisotropy in Mn-substituted cobalt ferrite. J. Appl. Phys. 2006, 99 (8), 08R102.

109. Eid, C.; Assaf, E.; Habchi, R.; Miele, P.; Bechelany, M., Tunable properties of GOdoped $\mathrm{CoFe} 2 \mathrm{O} 4$ nanofibers elaborated by electrospinning. RSC Advances 2015, 5 (118), 97849-97854.

110. Schwaminger, S. P.; García, P. F.; Merck, G. K.; Bodensteiner, F. A.; Heissler, S.; Günther, S.; Berensmeier, S., Nature of Interactions of Amino Acids with Bare Magnetite Nanoparticles. J. Phys. Chem. C 2015, 119 (40), 23032-23041.

111. Wang, L.; Zhuo, L.; Cheng, H.; Zhang, C.; Zhao, F., Porous carbon nanotubes decorated with nanosized cobalt ferrite as anode materials for high-performance lithiumion batteries. Journal of Power Sources 2015, 283, 289-299.

112. Xiong, P.; Chen, Q.; He, M.; Sun, X.; Wang, X., Cobalt ferrite-polyaniline heteroarchitecture: a magnetically recyclable photocatalyst with highly enhanced performances. J. Mater. Chem. 2012, 22 (34), 17485-17493.

113. Rieck, G. D.; Thijssen, J. J. M., The cation distribution in CoFe2O4. Acta Crystallographica Section B 1968, 24 (7), 982-983.

114. Sinha, A. K.; Singh, M. N.; Achary, S. N.; Sagdeo, A.; Shukla, D. K.; Phase, D. M., Crystal field splitting and spin states of Co ions in cobalt ferrite with composition Co1.5Fe1.5O4 using magnetization and X-ray absorption spectroscopy measurements. $J$. Magn. Magn. Mater. 2017, 435, 87-95.

115. Bengtson, A.; Morgan, D.; Becker, U., Spin state of iron in Fe3O4 magnetite and h-Fe3O4. Phys. Rev. B 2013, 87 (15), 155141.

116. Rogers, J. A.; Bao, Z.; Baldwin, K.; Dodabalapur, A.; Crone, B.; Raju, V. R.; Kuck, V.; Katz, H.; Amundson, K.; Ewing, J.; Drzaic, P., Paper-like electronic displays: Largearea rubber-stamped plastic sheets of electronics and microencapsulated electrophoretic inks. Proceedings of the National Academy of Sciences 2001, 98 (9), 4835-4840.

117. Sekitani, T.; Someya, T., Stretchable, Large-area Organic Electronics. Adv. Mater. 2010, 22 (20), 2228-2246.

118. Ukah, N. B.; Granstrom, J.; Sanganna Gari, R. R.; King, G. M.; Guha, S., Lowoperating voltage and stable organic field-effect transistors with poly (methyl methacrylate) gate dielectric solution deposited from a high dipole moment solvent. Appl. Phys. Lett. 2011, 99 (24), 243302.

119. Ukah, N. B.; Senanayak, S. P.; Adil, D.; Knotts, G.; Granstrom, J.; Narayan, K. S.; Guha, S., Enhanced mobility and environmental stability in all organic field-effect transistors: The role of high dipole moment solvent. J. Polym. Sci. Part B Polym. Phys. 2013, 51 (21), 1533-1542.

120. Cipriano, T.; Knotts, G.; Laudari, A.; Bianchi, R. C.; Alves, W. A.; Guha, S., Bioinspired peptide nanostructures for organic field-effect transistors. ACS applied materials \& interfaces 2014, 6 (23), 21408-15.

121. Tessler, N.; Denton, G. J.; Friend, R. H., Lasing from conjugated-polymer microcavities. Nature 1996, 382 (6593), 695-697.

122. Holmes, R. J., Optical materials: Nanowire lasers go organic. Nat Nano 2007, 2 (3), 141-142.

123. Heliotis, G.; Xia, R.; Turnbull, G. A.; Andrew, P.; Barnes, W. L.; Samuel, I. D. W.; Bradley, D. D. C., Emission Characteristics and Performance Comparison of Polyfluorene 
Lasers with One- and Two-Dimensional Distributed Feedback. Advanced Functional Materials 2004, 14 (1), 91-97.

124. O'Carroll, D.; Iacopino, D.; O'Riordan, A.; Lovera, P.; O'Connor, É.; O'Brien, G. A.; Redmond, G., Poly(9,9-dioctylfluorene) Nanowires with Pronounced $\beta$-Phase Morphology: Synthesis, Characterization, and Optical Properties. Advanced Materials 2008, 20 (1), 42-48.

125. Tovar, J. D., Supramolecular Construction of Optoelectronic Biomaterials. Accounts of Chemical Research 2013, 46 (7), 1527-1537.

126. Wall, B. D.; Zhou, Y.; Mei, S.; Ardoña, H. A. M.; Ferguson, A. L.; Tovar, J. D., Variation of Formal Hydrogen-Bonding Networks within Electronically Delocalized $\pi$ Conjugated Oligopeptide Nanostructures. Langmuir : the ACS journal of surfaces and colloids 2014, 30 (38), 11375-11385.

127. Martins, T. D.; de Souza, M. I.; Cunha, B. B.; Takahashi, P. M.; Ferreira, F. F.; Souza, J. A.; Fileti, E. E.; Alves, W. A., Influence of pH and Pyrenyl on the Structural and Morphological Control of Peptide Nanotubes. The Journal of Physical Chemistry C 2011, 115 (16), 7906-7913.

128. Souza, M. I.; Jaques, Y. M.; de Andrade, G. P.; Ribeiro, A. O.; da Silva, E. R.; Fileti, E. E.; Avilla Ede, S.; Pinheiro, M. V.; Krambrock, K.; Alves, W. A., Structural and photophysical properties of peptide micro/nanotubes functionalized with hypericin. $J$. Phys. Chem. B 2013, 117 (9), 2605-14.

129. Grice, A. W.; Bradley, D. D. C.; Bernius, M. T.; Inbasekaran, M.; Wu, W. W.; Woo, E. P., High brightness and efficiency blue light-emitting polymer diodes. Applied Physics Letters 1998, 73 (5), 629-631.

130. Knaapila, M.; Torkkeli, M.; Monkman, A. P., Evidence for 21-Helicity of Poly[9,9bis(2-ethylhexyl)fluorene-2,7-diyl]. Macromolecules 2007, 40 (10), 3610-3614.

131. Arif, M.; Volz, C.; Guha, S., Chain Morphologies in Semicrystalline Polyfluorene: Evidence from Raman Scattering. Physical Review Letters 2006, 96 (2), 025503.

132. Demirel, G.; Buyukserin, F., Surface-induced self-assembly of dipeptides onto nanotextured surfaces. Langmuir : the ACS journal of surfaces and colloids 2011, 27 (20), 12533-8.

133. Xu, L.; Zhang, J.; Peng, J.; Qiu, F., Formation of nanofibers in $\operatorname{Poly}(9,9-$ dioctylfluorene) toluene solutions during aging. Journal of Polymer Science Part B: Polymer Physics 2015, 53 (9), 633-639.

134. Li, Y.; Thouas, G. A.; Shi, H.; Chen, Q., Enzymatic and oxidative degradation of poly(polyol sebacate). Journal of biomaterials applications 2014, 28 (8), 1138-50.

135. Martins, R. F. P.; Ahnood, A.; Correia, N.; Pereira, L. M. N. P.; Barros, R.; Barquinha, P. M. C. B.; Costa, R.; Ferreira, I. M. M.; Nathan, A.; Fortunato, E. E. M. C., Recyclable, Flexible, Low-Power Oxide Electronics. Advanced Functional Materials 2013, 23 (17), 2153-2161.

136. Petritz, A.; Wolfberger, A.; Fian, A.; Griesser, T.; Irimia-Vladu, M.; Stadlober, B., Cellulose-Derivative-Based Gate Dielectric for High-Performance Organic Complementary Inverters. Advanced materials (Deerfield Beach, Fla.) 2015.

137. Denev, S. A.; Lummen, T. T. A.; Barnes, E.; Kumar, A.; Gopalan, V.; Green, D. J., Probing Ferroelectrics Using Optical Second Harmonic Generation. Journal of the American Ceramic Society 2011, 94 (9), 2699-2727. 


\section{PUBLICATIONS}

- Soma Khanra, Md Mamun, Fabio F. Ferreira, Kartik Ghosh, and Suchismita Guha, "Functionalized self-assembled peptide nanotubes with cobalt ferrite nanoparticles for application in organic electronics" ACS Appl. Nano Mater. 1, 3, 1175-1187 (2018).

- Sergio Kogikosk Jr, Soma Khanra, Sivoney F. Souza, Michelle S. Liberato, Wendel A. Alves, and Suchismita Guha, "SERS active self-assembled diphenylalanine micro/nanostructures: hexagonal versus the orthorhombic phase" J. Chem. Phys. 147, 084703 (2017).

- S. Khanra, K. Ghosh, F. F. Ferreira, W. A. Alves, F. Punzo. P. Yu, and S. Guha "Probing nonlinear optical coefficients of self-assembled peptide nanotubes Phys. Chem. Chem. Phys. 19, 3084 (2017).

- Alec Pickett, Abhisek Mohapatra, Amrit Laudari, Soma Khanra, Thulasi Ram, Satish Patil, and Suchismita Guha, "Hybrid ZnO-organic semiconductor interfaces in photodetectors: A comparison of two near-infrared donor-acceptor copolymers, Organic Electronics 45 115-123 (2017).

- S. Khanra, T. Cipriano, T. Lam, T. A. White, E. E. Fileti, W. A. Alves, and S. Guha, "Self-assembled Peptide-Polyfluorene Nanocomposites for Biodegradable Organic Electronics" Adv. Mater. Interfaces 2 (2015) 1500265. [selected as inside cover] 


\section{VITA}

Soma Khanra was born in a small village in West Bengal, India. Soma was graduated from West Bengal University of Technology (WBUT) with a bachelor's degree in Electronics and Communication Engineering (ECE) in 2009. She came to USA in 2011 to do Master in Materials Science at Missouri State University, Springfield. After completing her master degree in fall, 2012 from Missouri State University, she joined the Physics and Astronomy department at the University of Missouri, Columbia in spring, 2013. Soma will be graduating in May 2018 with a $\mathrm{PhD}$ degree in Physics under Prof. Suchismita Guha. 